\section{ADVANCES IN THE VISUALIZATION AND ANALYSIS OF BOUNDARY LAYER FLOW IN SWIMMING FISH}

By

\author{
Erik J. Anderson \\ M.S., Saint Francis Xavier University, 1998 \\ B.S., Gordon College, 1989 \\ Submitted in partial fulfillment of the requirements for the degree of \\ Doctor of Philosophy \\ at the \\ MASSACHUSETTS INSTITUTE OF TECHNOLOGY \\ and the \\ WOODS HOLE OCEANOGRAPHIC INSTITUTION
}

February 2005

(C) 2005 Erik J. Anderson. All rights reserved.

The author hereby grants to MIT and WHOI permission to reproduce paper and electronic copies of this thesis in whole or in part and to distribute them publicly.

Signature of Author

Joint Program in Oceanography/Applied Ocean Science and Engineering Massachusetts Institute of Technology

atd Woods Hole Oceanographic Institution

February 2005

Certified by

Mark A. Grosenbaugh

Thesis Supervisor

Certified by

Wade R. McGillis

Thesis Supervisor

Certified by

Mark A. Grosenbaugh

Chairman, Joint Committee for Applied Ocean Science and Engineering Woods Hole Oceanographic Institution 


\section{Page 2}

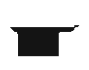




\title{
Advances in the visualization and analysis of boundary layer flow in swimming fish
}

\author{
By
}

Erik J. Anderson

\begin{abstract}
In biology, the importance of fluid drag, diffusion, and heat transfer both internally and externally, suggest the boundary layer as an important subject of investigation, however, the complexities of biological systems present significant and unique challenges to analysis by experimental fluid dynamics. In this investigation, a system for automatically profiling the boundary layer over free-swimming, deforming bodies was developed and the boundary layer over rigid and live mackerel, bluefish, scup and eel was profiled. The profiling system combined robotics, particle imaging velocimetry, a custom particle tracking code, and an automatic boundary layer analysis code. Over 100,000 image pairs of flow in the boundary layer were acquired in swimming fish alone, making spatial and temporal ensemble averaging possible.
\end{abstract}

A flat plate boundary layer was profiled and compared to known laminar and turbulent boundary layer theory. In general, profiles resembled those of Blasius for sub-critical length Reynolds numbers, $R e_{x}$. Transition to a turbulent boundary layer was observed near the expected critical $R e_{x}$ and subsequent profiles agreed well with the law of the wall. The flat plate analysis demonstrated that the particle tracking and boundary layer analysis algorithms were highly accurate.

In rigid fish, separation of flow was clearly evident and the boundary layer transitioned to turbulent at lower $R e_{x}$ than in swimming fish and the flat plate. Wall shear stress, $\tau_{i}$, forward of separation was slightly higher than flat plate values. Friction drag in rigid and swimming fish was determined by integrating $\tau_{o}$ over the surface of the fish. The analysis was facilitated by the definition of the relative local coefficient of friction. In general, there was no significant difference in friction drag between the rigid-body and swimming cases. In swimming, separation was, on average, delayed. Therefore, pressure drag was estimated on the basis of thickness ratio and used to calculate an upper-bound total drag on a swimming fish. Total drag was used to determine the required muscle power output during swimming and compare that with existing muscle power data. $\tau_{o}$ and boundary layer thickness oscillated with undulatory phase. The magnitude of oscillation appears to be linked to body wave amplitude. 
Page 4 


\section{Contents}

i. Acknowledgements

ii. List of figures

iii. List of tables

iv. List of symbols

\section{Introduction}

1.1 Definition of a boundary layer

1.2 History of boundary layer studies in fish swimming

1.3 The problem and history of drag measurement in undulatory swimming

1.4 Contribution of the present investigation

1.5 Chapter preview

\section{Boundary layer theory}

2.1 Laminar boundary layer solutions

2.2 Length Reynolds number, $R e_{x}$

2.3 Falkner-Skan laminar boundary layer solution

2.4 Turbulent boundary layer equations

2.5 The $1 / 7^{\text {th }}$ power turbulent boundary layer profile approximation

2.6 Turbulence intensity

2.7 Boundary layer thickness

2.8 Wall shear stress and friction drag 


\section{Preliminary investigation}

3.1 Methods and materials

3.1.1 Fish

3.1.2 Swimming conditions

3.1.3 Image acquisition

3.1.4 Rigid-body drag

3.1.5 Digital particle tracking velocimetry

3.1.6 Tangential and normal velocity calculations

3.1.7 DPTV errors

3.1.8 Undulatory phase

3.2 Results

3.2.1 Fish boundary layer profiles

3.2.2 Flow condition in the boundary layer

3.2.3 Local friction coefficients

3.2.4 Oscillatory behavior of the boundary layer

3.2.5 Oscillation of normal velocity

3.2.6 Incipient separation

3.2.7 Total skin friction and friction coefficients

\subsection{Discussion}

3.3.1 The nature of the fish boundary layer

3.3.2 Wave-like distributions of boundary layer variables and pressure

3.3.3 Drag enhancement and drag reduction

3.3.4 Drag reduction mechanisms

3.3.5 Two-dimensional analysis of a three-dimensional phenomenon

3.3.6 Power to overcome friction drag

3.3.7 The advantages of boundary layer visualization 


\section{Advances data acquisition}

4.1 Specimens and trials

4.2 Specimen collection and care

4.3 Flume test section

4.4 Strobe imaging of the flow

4.5 Robotic control of data acquisition

4.6 Automatic calibration

4.7 Automatic scanning of long video records for usable data

\section{Automatic boundary layer PTV and analysis}

5.1 The failure of conventional DPIV and DPTV to resolve the boundary layer

5.2 An automatic boundary layer profiling and analysis code

5.2.1 Automatic object surface edge detection

5.2.2 Surface tracking

5.2.3 Surface and glare removal

5.2.4 Particle centroiding

5.2.5 Particle tracking by track convergence velocimetry

5.3 Boundary layer profile calculation

5.4 Boundary layer profile analysis

5.5 The relative, local drag coefficient

5.6 Errors in the calculation of boundary layer parameters

5.7 Criteria for accurate boundary layer data

5.8 Undulatory phase 


\section{Experimental controls: flume profile, flat plate, rigid fish}

6.1 Flume profile

6.1.1 Flume wall boundary layers

6.1.2 Flume plug flow

6.1.3 Fluctuations of streamwise velocity in the plug flow

6.1.4 Summary of flume profile analysis

6.2 Flat plate boundary layer

6.2.1 Details of the flat plate experiments

6.2.2 The flat plate coordinate system

6.2.3 Comparison of upstream profile with flume plug profile

6.2.4 General observations of the flat plate boundary layer

6.2.5 Boundary layer thickness

6.2.6 Errors in estimating velocity gradient at the plate surface

6.2.7 Velocity gradient and local coefficient of friction on the flat plate

6.2.8 Relative local coefficient of friction, $C_{f x R}$, on the flat plate

6.3 Boundary layer flow over rigid fish

6.3.1 Boundary layer separation in rigid fish

6.3.2 Early transition to a turbulent boundary layer

6.3.3 Friction on a rigid fish

\section{The boundary layer of swimming fish}

7.1 Body wave amplitude and frequency

7.2 The bluefish boundary layer

7.2.1 Bluefish boundary layer thickness

7.2.2 Bluefish boundary layer local coefficient of friction

7.2.3 Significance of bluefish boundary layer findings

7.3 The scup boundary layer 
7.3.1 Scup boundary layer thickness

7.3.2 Scup boundary layer local coefficient of friction

7.3.3 Significance of scup boundary layer findings

7.4 The eel boundary layer

7.4.1 Eel boundary layer thickness

7.4.2 Eel boundary layer local coefficient of friction

7.4.3 Significance of eel boundary layer findings

7.5 The mackerel boundary layer

7.5.1 Mackerel boundary layer thickness

7.5.2 Mackerel boundary layer local coefficient of friction

7.5.3 Significance of mackerel boundary layer findings

7.6 Drag and power in swimming fish

7.6.1 Comparison of local friction in rigid and swimming fish

7.6.2 Total friction coefficients and total friction drag on swimming fish

7.6.3 Estimated pressure drag and an upper-bound overall drag

7.6.4 Power requirements and available muscle power

\section{Conclusions and suggestions for future work}

8.1 The boundary layer of swimming fish

8.2 The drag on swimming fish

8.3 Improvements in boundary layer profiling

8.3.1 Improved temporal and spatial resolution

8.3.2 Machine vision

8.4 Boundary layers in biology-a new frontier

\section{References}


Page 10 


\section{i. Acknowledgements}

The author is grateful for the funding received from the Office of Naval Research, Grants N00014-99-1-1082 and N00014-96-1141, the MRI program of the National Science Foundation, Grant OCE-9724383, the National Science Foundation Graduate Research Traineeship in Coastal Oceanography, the WHOI Ocean Ventures Fund, the WHOI Academic Programs Office, and the MIT Department of Ocean Engineering.

The author thanks his thesis supervisors Mark Grosenbaugh and Wade McGillis for their guidance and enthusiasm though the entire course of this extensive experimental work. The author also thanks Mike Triantafyllou, whose feedback concerning fish hydrodynamics played an important role in guiding the inquiry. The author thanks Larry Rome (UPenn) for his contributions as an external committee member and his critique of the work from a biological perspective. The author also thanks John Farrington, Judy McDowell, Paola Rizzoli, Ronni Schwartz, Marsha Gomes, Julia Westwater, Marcey Simon, Stacey Brudno Drange, Stephen Malley, Dominique Jeudy, and the rest of the administrative staff of the MIT/WHOI Joint Program and the Ocean Engineering student administrative staff for their dedication to the success of all the students in the Joint Program. The author thanks the faculty and staff of the MIT Department of Ocean Engineering and the WHOI Department of Applied Ocean Physics and Engineering. The author thanks John Trowbridge for serving as defense chair.

Many others made invaluable contributions to this project. The author thanks Kyle McKenney (MIT, UROP) for three summers of help 'collecting specimens', meticulously cataloging thousands of images and data files, and monitoring data analysis. The author thanks Jay Sisson for engineering support and his management of the resources of the Rhinehart Coastal Research Center. The author also thanks Bruce Tripp and Bebe McCall for their administrative support at RCRC. The author thanks Rick Galat, Fred Keller, Bruce Lancaster, Joe Connolly, Mike Cifelli, and all the staff responsible for the functioning of the experimental facility. The author also thanks Ann Stone, Nancy Stedman, Keith Bradley, Houshuo Jiang, Sean McKenna, Steve Fries, Debbie Schafer, Fred Baker, Eileen Wicklund, Arden Edwards, Oscar Pizarro, Mark Rapo, Scott Gallager, Bruce Woodin, WHOI Housing and WHOI Security.

Finally, the author thanks his parents, sisters, family, friends, his wife, Rachel Anderson, and the Lord, Jesus Christ, for encouragement and strength. 


\section{ii. List of figures}

1.1 Illustrative boundary layer profiles

2.1 Turbulent and laminar flat plate boundary layer scaled according to law of the wall

3.1 Set-up for flow visualization

3.2 Double exposure showing particle pairs

3.3 Fish boundary layer profiles compared to Blasius and the law of the wall

3.4 Fish boundary layer profiles compared to Falkner-Skan

3.5 Time-averaged local coefficient of friction on swimming fish

3.6 Boundary layer parameters vs. fish body position

3.7 Time-averaged local coefficient of friction for scup at $x / L=0.5$

3.8 Local coefficient of friction and boundary layer thickness vs. body phase

$3.9 U_{e}$ and $V_{e}$ vs. body phase

3.10 Time series of normal velocity profiles on swimming fish

3.11 Boundary layer development over scup swimming in still water

3.12 Boundary layer profile time series showing incipient separation

3.13 Friction coefficients on swimming fish

4.1 Scaled sideview images of four fish species examined

4.2 Image of flume

4.3 Image of flume test section

4.4 Laser and sideview camera robot

4.5 Boundary layer and nearfield camera robot

5.1 Illustration of DPIV

5.2 Illustration of conventional DPTV

5.3 Dlustration of the 'forced-monotonic' filter for near surface glare determination

5.4 Computer generated image pair used in description of particle tracking code

5.5 Plot of all potential particle tracks

5.6 Plot of track distance vs. track angle for all potential tracks

5.7 The track plot image, or matrix

5.8 Kernel multiplication for density score

5.9 Track selection process

5.10 Results of particle tracking example

5.11 Particle tracks and profiles for flow $10 \mathrm{~cm}$ upstream of the flat plate

5.12 Velocity field for flow $10 \mathrm{~cm}$ upstream of the flat plate

5.13 Particle tracks and profiles for flow at $x=21.3 \mathrm{~cm}$ on the flat plate

5.14 Velocity field for flow at $x=21.3 \mathrm{~cm}$ on the flat plate

5.15 Particle tracks and profiles for flow at $x=32.3 \mathrm{~cm}$ on a swimming bluefish

5.16 Velocity field for flow at $x=32.3 \mathrm{~cm}$ on a swimming bluefish

5.17 Comparison of conventional DPIV and PTCV

5.18 Transverse body surface motion from nearfield camera view 
6.1 Flume test section profiles at $98 \mathrm{~cm} / \mathrm{s}$, blank inlet barrier

6.2 Flume test section profiles at $98 \mathrm{~cm} / \mathrm{s}$, large grid inlet barrier

6.3 Power spectra of fluctuations in mean flume profiles

$6.4 u$ velocity field near inlet with the large grid barrier

6.5 Boundary layer profiles over flat plate, $U=33.0 \mathrm{~cm} / \mathrm{s}$, blank inlet barrier

6.6 Boundary layer profiles over flat plate, $U=68.7 \mathrm{~cm} / \mathrm{s}$, blank inlet barrier

6.7 Boundary layer profiles over flat plate, $U=117 \mathrm{~cm} / \mathrm{s}$, blank inlet barrier

6.8 Boundary layer $u$-profile at $x=29.4$ on flat plate fit to the law of the wall

6.9 Boundary layer profiles over flat plate, $U=117 \mathrm{~cm} / \mathrm{s}$, small grid inlet barrier

6.10 Coefficient of friction vs. length Reynolds number for all flat plate experiments

6.11 Relative coefficient of friction vs. relative position for flat plate data

6.12 Relative coefficient of friction vs. relative position showing grid effect on transition

6.13 Boundary layer profiles for all rigid fish species showing separation

6.14 Boundary layer profiles for all rigid fish species showing transition to turbulence

6.15 Relative coefficient of friction vs. relative position for all rigid fish cases

7.1 Definition of body phase and phase bins for ensemble profiles in live fish

7.2 Body wave amplitude vs. body position and swimming speed

7.3 Comparison across species of body wave amplitude vs. body position

7.4 Transverse body velocity at trailing edge and body wave frequency

7.5 Live bluefish boundary layer profiles

7.6 Fluctuation in boundary layer thickness in bluefish

7.7 Fluctuation of local friction in bluefish

7.8 Live scup boundary layer profiles

7.9 Phase plots of $\delta_{95}$ and $C_{f x}$ in scup

7.10 Live eel boundary layer profiles

7.11 Phase plots of $\delta_{95}$ and $C_{f x}$ in eel

7.12 Live mackerel boundary layer profiles, 'high' speed

7.13 Phase plots of $\delta_{95}$ and $C_{f x}$ in mackerel

7.14 Live mackerel boundary layer profiles, 'low' speed

7.15 Summary of live and rigid fish $C_{f x R}$ vs. body position

7.16 Summary plot of $C_{f}$ vs. $R e$

7.17 Power required to overcome drag vs. swimming speed 


\section{iii. List of tables}

3.1 A comparison of power requirements and availability in scup

4.1 Summary of conditions for all experiments and experimental controls

7.1 Friction data for swimming fish, laminar boundary layer

7.2 Friction data for swimming fish, turbulent boundary layer

7.3 Friction drag as a percentage of total drag based on thickness ratio

7.4 Comparison of transitional length Reynolds numbers

7.5 Power required per mass of red muscle, laminar boundary layer

7.6 Power required per mass of red muscle, turbulent boundary layer 


\section{vi. List of symbols}

A total wetted surface area of the body or body wave amplitude

$c$ body wave speed or chord length

$C_{f} \quad$ coefficient of friction

$C_{f t}$ theoretical friction drag coefficient

$C_{f x} \quad$ local coefficient of friction

$C_{f x B} \quad$ local coefficient of friction from Blasius

$C_{f x R}$ relative local coefficient of friction

$C_{f x} T$ local coefficient of friction from $1 / 7^{\text {th }}$ power turbulent profile

$\mathrm{Cl}_{95} \quad 95 \%$ confidence interval of the mean

$d A \quad$ incremental area on the body surface

$d y_{\max }$ maximum expected $y$-direction travel of a particle in pixels

$D_{f} \quad$ total skin friction

$D_{f t} \quad$ theoretical rigid body friction drag

$f \quad$ frequency of the body wave; generic function name

$h \quad$ step size in numerical differential equation solver

$i \quad$ index $=0,1,2, \ldots$; matrix row index

$I$ turbulence intensity

$I_{x} \quad x$-direction turbulence intensity

$j \quad$ index $=0,1,2, \ldots ;$ matrix column index

$\ell \quad$ streamwise dimension of the field of view of the particle imaging camera

$\ell_{s} \quad$ length of a body surface sample

$\mathbf{L}$ designates a curve representing friction on a flat plate with a laminar boundary layer

$L \quad$ body length; fork length in fish

$L_{c} \quad$ normalized streamwise chord length

$m \quad$ shape constant in Falkner-Skan boundary layer solution

$M \quad$ total wet mass of fish

$n \quad$ number of samples; number of particles

$n \quad$ unit normal vector of the body surface

$P \quad$ position of particle centroid

$r \quad$ position

$R, R_{i j}$ distance from the center $(i, j)$ entry of a kernel

$R^{2} \quad$ coefficient of determination for a given regression

$R e \quad$ Reynolds number based on body length

$R e_{\kappa} \quad$ roughness Reynolds number, i.e. based on average roughness height, $\kappa$

$R e_{x} \quad$ length Reynolds number, i.e. based on $x$

$S \quad$ the function describing the body surface

St Strouhal number

$t \quad$ time or body thickness 


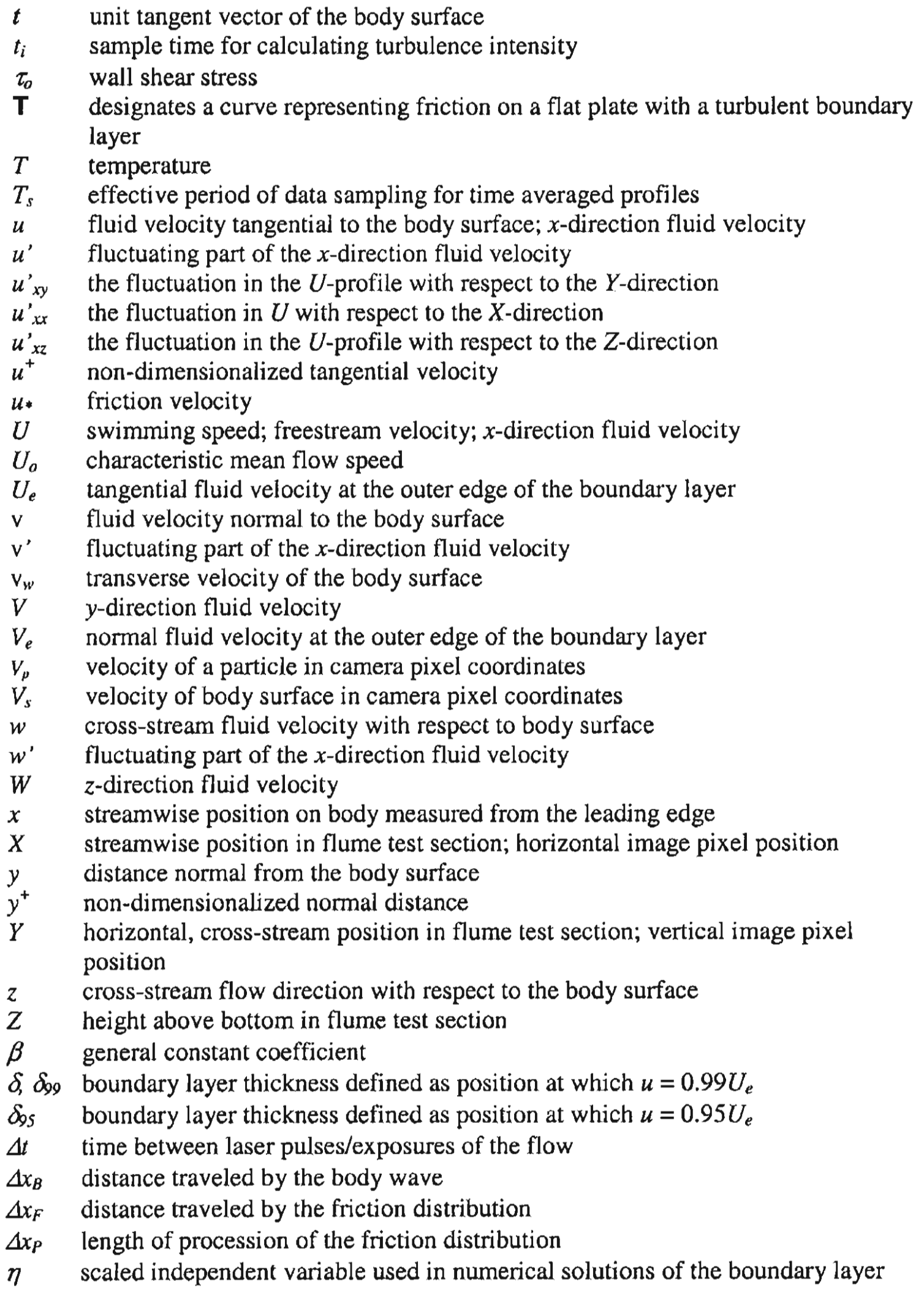


$\theta$ angle between the body surface tangent and swimming direction

$\kappa \quad$ average roughness height

$\lambda \quad$ wavelength of the friction distribution

$\lambda_{B} \quad$ wavelength of the body wave

$\mu \quad$ dynamic viscosity

$v \quad$ kinematic viscosity

$\rho \quad$ fluid density

$\sigma \quad$ standard deviation

$\sigma^{2} \quad$ variance

$\phi \quad$ body phase

$\phi_{o} \quad$ procession of distribution wave through the body wave per body length

$\psi \quad$ stream function 

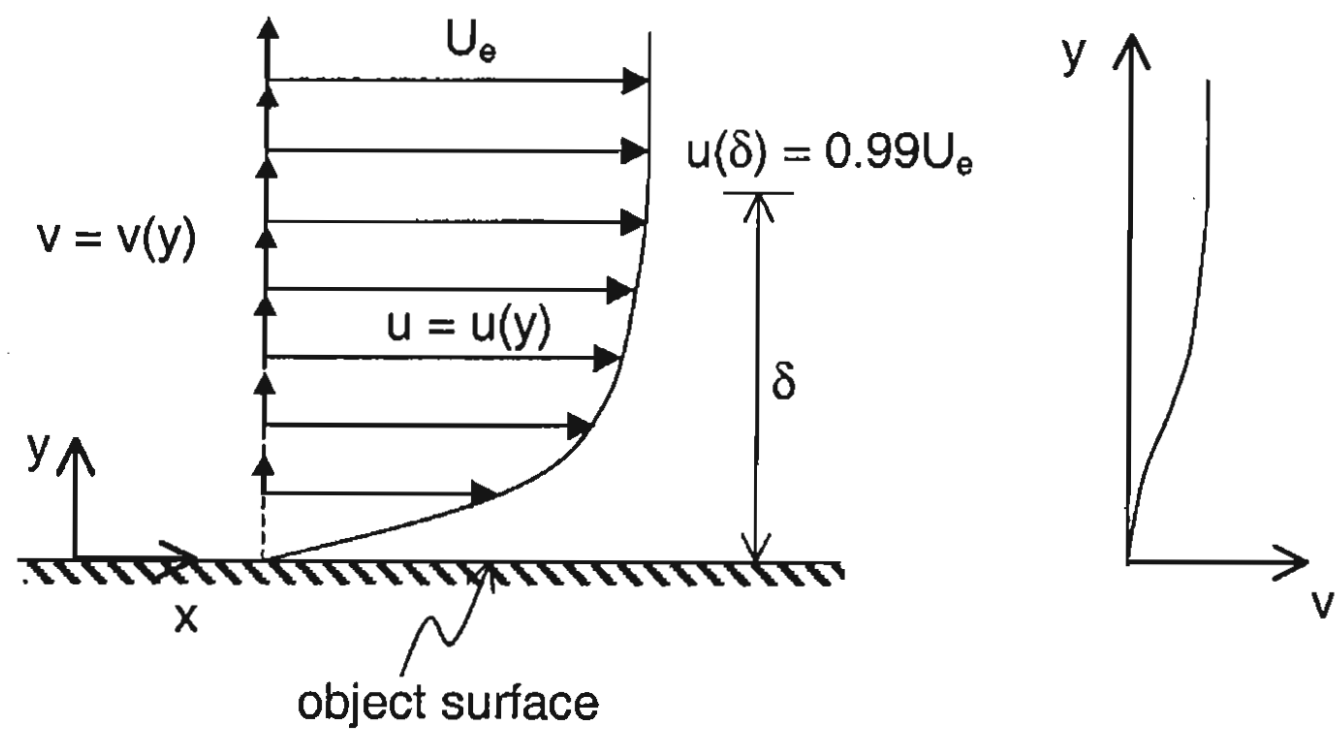

Fig. 1.1 Tangential and normal velocity profiles in the boundary layer over the surface of an object. Horizontal vectors represent tangential velocities and vertical vectors, normal velocities. Tangential velocity, $u$, above any given position, $x$, along the surface varies from 0 to $U_{e}$ with normal distance, $y$, from the surface. Boundary layer thickness, $\delta$, is defined as the normal distance between the surface of the object and the point at which $u=0.99 U_{e}$. The curve connecting the tips of the tangential velocity vectors is known as the $u$-profile. The plot of $v$ as a function of $y$, displayed to the right of the diagram demonstrates the conventional presentation of the normal velocity profile, or v-profile. There would be a set of velocity profiles for every position, $x$, along the surface of the object in this two dimensional example. It is important to note that all velocities are measured with respect to a coordinate system fixed to the body surface. Therefore, the same basic profile shapes are obtained whether the object is held stationary in a flow or if the object moves through still water. 


\section{Chapter 1}

\section{Introduction}

\subsection{Definition of a boundary layer}

When a body moves relative to a surrounding fluid, a boundary layer exists very close to the body surface due to the 'no-slip condition' and viscosity (Prandtl, 1904). Consider an object held stationary in a uniform oncoming flow with velocity $U$. The fluid in direct contact with the body surface adheres to the surface and has zero velocity. The fluid just above the surface is slowed by frictional forces associated with the viscosity of the fluid. The closer the fluid is to the surface, the more it is slowed. The result is a thin layer where the tangential velocity, $u$, of the fluid increases from zero at

the body surface to a velocity close to $U$. This velocity at the outer edge of the boundary layer, $U_{e}$, depends on the shape of the body (Schetz, 1993). By definition, the boundary layer extends from the object surface, $y=0$, to a position $y=\delta$, where the tangential velocity relative to the object surface is $0.99 U_{e}$. The curve representing the continuous variation in tangential velocity from $y=0$ to $y=\delta$ is commonly referred to as the boundary layer profile, or more specifically the $u$-profile (Fig. 1.1). Normal velocity relative to the surface also varies from zero at the body surface to some external value, $V_{e}$, generating what is known as the v-profile (Fig. 1.1). A third profile, the $w$-profile, usually exists in the flow over three-dimensional surfaces, where $w$ is tangential to the wall and perpendicular to $u$. Note, that if $u, v$, or $w$, is not specified, the term 'boundary layer profile' generally refers to the $u$-profile.

The shapes of the boundary layer profiles above a particular position on a surface depend on the shape of the body, surface roughness, upstream history of the boundary layer, the surrounding flow field and Reynolds number. Flow condition in the boundary 
layer can be laminar or turbulent resulting in radically different classes of profile shapes. Prandtl (1952), Schlichting (1979), and Batchelor (1967) provide thorough descriptions of the boundary layer concept. The behavior of a body moving relative to a real fluid cannot be accurately described without an understanding of the boundary layer. Since Prandtl (1904), great strides have been made been made in understanding fluid forces acting on bodies. Nevertheless, the hydrodynamics of undulatory swimming remain elusive. Drag, thrust and power in undulatory swimming have not been definitively determined. This is, in part, due to the fact that no definitive measurements of boundary layer flow over a swimming fish or cetacean have been performed.

\subsection{History of boundary layer studies in fish swimming}

Few attempts have been made to characterize the boundary layers of undulatory swimmers, and none have produced boundary layer velocity profiles. Most recently, Rohr et al. (1998a) have suggested that the relative intensity of bioluminescence around a swimming dolphin may be linked to the thickness of the boundary layer. In a set of earlier investigations, Kent et al. (1961) and Allen (1961) achieved a qualitative description of flow in the nearfield and possibly the boundary layers of fish using the Schlieren technique. The nearfield is the region of flow around the fish affected by the presence of the fish and its swimming motions. In contrast, the so-called far-field is the region in which the impact of the fish has decayed essentially to zero. While the boundary layer can certainly be considered part of the nearfield flow, to aid in the discussion, the term nearfield will be used here to refer to the region dominated by the presence of the fish, but outside of the boundary layer.

\subsection{The problem and history of drag measurement in undulatory swimming}

The understanding of drag mechanisms in undulatory swimming has been impeded significantly by this lack of boundary layer data. Both form drag and friction 
drag on a body depend on the nature of the boundary layer. Unlike the drag on a rigid body, such as an airplane wing, the drag on a swimming fish cannot be measured by simply placing a fish-shaped model in a wind or water tunnel. The boundary layer of a swimming fish is complicated by the motion of the body, and is certainly different than that over a rigid model. Furthermore, since the drag and thrust producing mechanisms of a swimming fish are coupled, even the use of an actively swimming model requires indirect means to determine drag (Barrett et al., 1999). Gray (1936) was clearly skeptical of the extension of the so-called 'rigid-body analogy' to the determination of drag on a swimming dolphin, but, left with no alternative, he used rigid-body drag as a tentative approximation. Webb (1975) catalogues the rigid-body drag calculations and measurements on fish that ensued, but reiterates the warning concerning the weakness of the analogy. The reservations of Gray were affirmed when Lighthill $(1960,1970,1971)$ published his reactive model of fish propulsion, which predicted thrust in steady swimming to be as much as 3 - 5 times greater than the theoretical rigid-body drag. This suggests that the drag on a steadily swimming fish is $3-5$ times greater than rigid-body drag. While Lighthill's reactive thrust model is considered to overestimate thrust, it is widely believed that the drag on a swimming fish is, indeed, greater than rigid-body drag. With this in mind, Weihs (1974) determined that some fish might reduce energy costs by burst and coast swimming.

Lighthill (1971), citing discussions with Q. Bone, claims that the 'enhanced drag' in fish swimming may be the result of boundary layer effects resulting from the lateral movements of the body segments of swimming fish. The production of vorticity that occurs as the body surface is thrust into the surrounding fluid is likely to be higher than the outward diffusion of vorticity that occurs during the retreat of the body surface. The result of this mechanism would be a boundary layer that is thinner and of higher shear than would be expected over the rigid body. This suggests that higher friction drag is the source of the alleged enhanced drag. 
Lighthill's prediction of enhanced friction drag further confused the already troubled field of energetics in undulatory locomotion. Gray (1936) and Gero (1952), among others (see Webb, 1975), made measurements that suggested that the power required to overcome rigid-body drag for porpoises and certain fish was greater than their muscle mass was capable of producing. This spawned a search for mechanisms that could reduce the drag on an undulatory swimmer to levels below the rigid-body drag. If, as Lighthill suggested, the drag on a swimming fish is actually much greater than rigidbody drag, the energetics problem becomes more difficult to explain. It was clear that either (1) Lighthill's model over-predicted thrust, (2) swimming performances had been exaggerated, or (3) the estimates of available muscle power were too low.

Investigators of undulatory swimming hydrodynamics and muscle physiology have studied each of these alternatives in an attempt to resolve the discrepancies. Thrust and power were estimated from velocity measurements of the wake of a swimming mullet (Müller et al., 1997). The investigators used techniques that were developed to calculate thrust and minimum muscle power output in bird and insect flight, where they were met with varied success (Rayner, 1979a,b; Ellington, 1984; Spedding et al., 1984; Spedding, 1986, 1987). In their preliminary work, Müller et al. (1997) report thrust estimates even higher than the theoretical values of Lighthill (1971). At the same time, claims of extraordinary performances of undulatory swimmers have been toned down or qualified (Lang, 1974; Lighthill, 1969; Rohr et al., 1998b) and estimates of available muscle power have been refined (Bainbridge, 1961; Webb, 1975; Weis-Fogh and Alexander, 1977; Fish, 1993; Rome et al., 1993; Coughlin et al., 1996). In general, recent findings suggest that it is less incumbent upon fish and cetaceans to possess extraordinary drag reducing secrets (Lang, 1974; Fish and Hui, 1991). Still, the problem has not been unequivocally resolved. Experiments on excised fish muscle driven at rates equal to those measured in vivo have resulted in relatively low power outputs (Rome and Swank, 1992; Coughlin et al., 1996; Swank and Rome, 2000; Rome et al., 2000). These studies suggest that maximum power output measurements recorded during non- 
physiological stimulation and strain are not applicable in vivo. Furthermore, friction drag on swimming fish has continued to go unmeasured.

Despite the dearth of available boundary layer data and Lighthill's prediction of drag enhancement based on theoretical thrust, theories of drag reduction by boundary layer manipulation abound. The most notable mechanisms proposed fall under the categories of laminar boundary layer maintenance, turbulent drag reduction, utilization of shed vorticity and the delay of separation. Theories of drag reduction in undulatory swimming are reviewed and critiqued in Webb (1975), Webb and Weihs (1983), and Fish and Hui (1991). One recent experimental work using a robotic fish claims to have substantiated drag reduction in undulatory swimming (Barrett et al., 1999). Earlier works, on the flow over waving plates, have also demonstrated mechanisms that may act to reduce drag, especially form drag. Taneda and Tomonari (1974) observed that the flow over a waving plate with wave speed, $c$, less than the oncoming flume speed, $U$, resulted in separation of flow and turbulent recirculation regions in the wave troughs. When wave speed was increased so that $c / U \geq 1$ flow remained attached over the entire plate. In some cases, boundary layer flow was completely laminarized. In others, it oscillated between turbulent and laminar.

\subsection{Contribution of the present investigation}

This thesis documents the first description of boundary layer flow in live swimming fish based on high-resolution velocity profiles acquired by flow visualization. Preliminary experiments were preformed using a highly manual data acquisition and analysis system (Anderson, McGillis, and Grosenbaugh, 2001). The methods and findings of these experiments are included here. However, the primary focus of this thesis is the data collected by an automated boundary layer profiling system developed by the author. The manual techniques of the preliminary work proved to be too time intensive to produce adequate data sets needed to make definitive conclusions regarding 
the fish boundary layer. The automated boundary layer profiling system increased the number of boundary layer realizations from 270 in two fish species to almost 200,000 in four species, including swimming and rigid-body cases. Thousands of systematic realizations were also determined for flow over a flat plate. The major contributions of this work include the design of the automated boundary layer profiling system and the findings regarding fish boundary layers coming from this large data set. The automated system, which includes highly efficient data acquisition, a novel particle tracking algorithm for flow visualization, and a boundary layer profile analyzer, is applicable to boundary layer profiling in general. In addition, the robotic data acquisition system is a valuable tool for general flow visualization around freely swimming organisms. The entire system is described in detail.

From fish boundary layer profiles, the unsteady spatial distribution of boundary layer related variables over the surface of swimming fish are determined. The distribution of wall shear stress, is used to estimate the total friction drag and the power necessary to overcome it. Theories of boundary layer manipulation, drag reduction, and friction drag enhancement are re-examined.

\subsection{Chapter preview}

Chapter 2 presents a general theoretical discussion for the reader not well versed in boundary layer theory. Those familiar with this branch of fluid dynamics may, therefore, skip Chapter 2. Chapter 3 is an abridged and updated version of the Methods and Materials, Results and Discussion of the preliminary experiments by Anderson, McGillis, and Grosenbaugh (2001). The most significant changes are (1) the revision and minor correction of the discussion concerning the wave-like distributions of boundary layer parameters over the length of the fish (section 3.3.2), and (2) the addition of comments regarding power requirements at high speeds in scup. Chapter 4 focuses on data acquisition, from the experimental subjects and conditions to a details description of 
the design and function of the robot-assisted image acquisition system. Chapter 5 presents the details of the automatic code developed by the author to extract boundary layer profiles from the acquired flow images and then analyze those profiles. The chapter features the particle tracking algorithm and the definition of a relative, local coefficient of friction that facilitates comparisons of wall shear stress distributions along swimming fish. Chapter 6 presents an important test of the boundary layer profiling codecharacterization of the flow over a flat plate--and the experimental controls: (1) characterization of the flow in the flume, and (2) the measurement of the boundary layer over rigid fish stretched straight in the flow. In Chapter 7, the results of boundary layer visualization in swimming fish are presented and comparisons are made between the results from the various species of fish observed. Finally, Chapter 8 deals with plans for future research and the next generation of the boundary layer profiling system. 
Page 26 


\section{Chapter 2}

\section{Boundary layer theory}

\subsection{Laminar boundary layer solutions}

Boundary layer profiles of the flow over various objects have been determined over the years both theoretically using the Navier-Stokes equations and experimentally using techniques such as hot-wire anemometry. Prandtl's student Blasius (1908) determined the first boundary layer solution from the Navier-Stokes equations. Blasius used numerical methods to determine the velocity profiles for the simplest flow geometry - steady laminar flow over a flat plate with no streamwise pressure gradient. These conditions and experimental results allowed him to reduce the Navier-Stokes equation to a differential equation of the form,

$$
f^{\prime \prime \prime}(\eta)+f(\eta) f^{\prime \prime}(\eta)=0
$$

where

$$
\begin{aligned}
& \eta=y \sqrt{\frac{U_{e}}{2 v x}} \\
& f(\eta)=\frac{\psi}{\sqrt{2 v U_{e} x}} \\
& f^{\prime}(\eta)=\frac{u}{U_{e}} \\
& \eta f^{\prime}(\eta)-f(\eta)=v \sqrt{\frac{2 x}{\nu U_{e}}}
\end{aligned}
$$

$y$ is the height above the flat plate, $x$ is the distance from the leading edge, $v$ is the kinematic viscosity of the fluid, and $\psi$ is the stream function (Schetz, 1993). The Blasius 
profile shape is self-similar, i.e. the shape of $y / \delta \mathrm{vs.} u / U_{e}$ is the same for all Blasius boundary layer profiles. Blasius used inner and outer series expansions to solve this equation, but it can also be solved easily using a shooting method whereby guesses are made for $f^{\prime \prime}(0)$ until boundary conditions are satisfied (Schetz, 1993). In this investigation, a $3^{\text {rd }}$ order Taylor Series shooting method (step size, $h=0.01$ ) was employed (Cheney and Kincaid, 1994). Blasius' solution shows excellent agreement with experimental data of boundary layer flow over flat plates and results in a set of simple equations that describe the important parameters. These equations are,

$$
\begin{aligned}
& \delta_{99}=4.9 x R e_{x}^{-1 / 2} \\
& \tau_{0}=0.332 \rho U^{2} R e_{x}^{-1 / 2}
\end{aligned}
$$

where $\delta_{99}$ is boundary layer thickness, $x$ is streamwise distance from the leading edge, $R e_{x}$ is the length Reynolds number, $\tau_{o}$ is wall shear stress, $\rho$ is the density of the fluid and $U$ is the freestream flow speed.

\subsection{Length Reynolds number, $\operatorname{Re}_{\mathrm{x}}$}

In the discussion of boundary layer data it is convenient to use a quantity know as the 'length Reynolds number', or $R e_{x}$. $R e_{x}$ is the Reynolds number based on position, $x$, that is

$$
\operatorname{Re}_{x}=\frac{U x}{v}
$$

Boundary layer thickness, wall shear stress and the transition of boundary layer flow from laminar to turbulent are generally dependent on $R e_{x}$ (Fox and McDonald, 1992). For example, the position at which laminar flow transitions to turbulent flow over a flat 
plate does not depend on the total length, $L$, of the plate. Instead, transition tends to occur at $R e_{x}=3.5 \times 10^{5}-5 \times 10^{5}$, for any flat plate or relatively similar surface (Schlichting, 1979), regardless of $L$ or the standard Reynolds number, $R e$, based on total length, $L$. Note that $R e_{x}$ at $x=L$ is the same as $R e$.

$R e_{x}$ is not universally applicable in analyzing fish swimming, however, since it fails to account for differing body shapes and body wave amplitude as a function of distance from the leading edge of the fish. Therefore, in several instances boundary layer parameters will be compared using position relative to fork length, i.e. $x / L$.

\subsection{Falkner-Skan laminar boundary layer solution}

Since Blasius, several other so-called exact solutions of the Navier-Stokes equations have been determined for laminar boundary layers, including accelerating and decelerating flows (Falkner and Skan, 1930), and three-dimensional flows (Sowerby, 1959). The former, or Falkner-Skan solution, arises from simplifications that allow for the reduction of the Navier-Stokes equation to

$$
f^{\prime \prime \prime}(\eta)+f(\eta) f^{\prime \prime}(\eta)-\frac{2 m}{m+1}\left(f^{\prime 2}(\eta)-1\right)=0
$$

where

$$
\begin{aligned}
& \eta=y \sqrt{\frac{m+1}{2} \frac{U_{e}}{v x}} \\
& f(\eta)=\sqrt{\frac{m+1}{2}} \frac{\psi}{\sqrt{v U_{e} x}}
\end{aligned}
$$

and $2 m \pi /(m+1)$ is the angle of a wedge over which the determined boundary layer profile would be expected to occur (Schetz, 1993). This equation can also be solved using a shooting method. The value of $m$ ranges from 1 for a stagnation flow, that is at right 
angles to a flat plate, 0 for a Blasius boundary layer, and -0.0904 for an inflected profile with $\tau_{o}=0$. The $u$-profile shape for $m>1$ is steeper than Blasius and for $m<1$ the profile is more gradual when plotted as $y / \delta_{9 g}$.vs. $u / U_{e}$. The Falkner-Skan solution is self-similar, but only for the same $m$. Therefore it is not possible to write a set of simple equations governing $\delta_{9 g}$ and $\tau_{o}$ such as Eq. 2.3 for all $m$. However, modern computing power can solve Eq. 2.5 in a small fraction of a second. Therefore, Falkner-Skan profiles can be easily calculated for curve fitting and other analyses. Furthermore, once the equation is solved for a given $m$, the value $f^{\prime \prime}(0)$ can be saved in a look-up table to speed future calculations. In this investigation a $4^{\text {th }}$ order Runga-Kutta method (Cheney and Kincaid, 1994) was employed to solve Eq. 2.5 (step size, $h=0.01$ ).

\subsection{Turbulent boundary layer equations}

Knowledge of turbulent boundary layer profiles comes mainly from experimental data. Time averaged measurements of turbulent flow over flat plates with no pressure gradient have conveniently revealed a universality known as the 'law of the wall' (Schlichting, 1979). When appropriately non-dimensionalized, the tangential velocity data follow a universal profile. The effects of streamwise pressure gradients and various geometries on this universal profile are well documented (Schetz, 1993). Tangential velocity, $u$, and distance from the wall, $y$, are non-dimensionalized for the law of the wall using,

$$
\begin{aligned}
& u^{+} \equiv \frac{u}{u_{*}} \quad u_{*} \equiv \sqrt{\frac{\tau_{o}}{\rho}} \\
& y^{+} \equiv \frac{y u_{*}}{v}
\end{aligned}
$$

where $\nu$ is the kinematic viscosity of the fluid, $\tau_{o}$ is the wall shear stress and $\rho$ is the fluid density. The defined intermediate, $u_{*}$, is known as the friction velocity. Traditionally, 
the non-dimensionalized tangential velocity, $u^{+}$, is plotted as a function of $\log _{10}\left(y^{+}\right)$. Fig. 2.1A shows the law of the wall plotted in this manner. Two distinct curves are evident. Closest to the wall, which can be thought of as running parallel to the $u^{+}$axis, the profile is linear, with $u^{+}=y^{+}$. Note that on a semi-logarithmic plot the relationship does not look linear. This curve represents the linear sublayer, which is commonly referred to as the viscous sublayer in the analysis of turbulent boundary layers. Farther from the wall, the profile follows a logarithmic curve. Flow is turbulent in the logarithmic region and laminar in the linear sublayer; a region called the transition zone separates the two. Unlike the linear sublayer, the shape and position of the logarithmic region of the time averaged profile may very significantly as a result of surface roughness and streamwise pressure gradients (Schetz, 1993). For this reason, data in the logarithmic region cannot be used to determine wall shear stress on an undulating fish. The linear sublayer must be used. Nevertheless, the general shape of the logarithmic region is still useful to distinguish between turbulent and laminar profiles. Boundary layer profiles were fit to the law of the wall using the linear sublayer when possible. The profile was then classified as turbulent or laminar based on the profile shape outside the linear sublayer. For example, if the Blasius boundary layer is plotted using the non-dimensionalization of Eq. 2.7, the majority of the boundary layer profile follows the linear curve and is poorly fit by the logarithmic curve (Fig. 2.1B).

It should be noted here that for turbulent boundary layers, it is the time-averaged profile at a given streamwise position that is described by the law of the wall. This dependence of the analysis of turbulence on sampling time is due to the fluctuating nature of turbulent flow. If the sampling time is too short, the instantaneous boundary layer profile could appear to be laminar-and not necessarily Blasius-like--even if the flow were turbulent. It is only when several instantaneous boundary layer profiles over a particular point in a turbulent boundary layer are drawn overlapped, that the average curve drawn through the combined profiles follows the law of the wall. Profiles acquired 

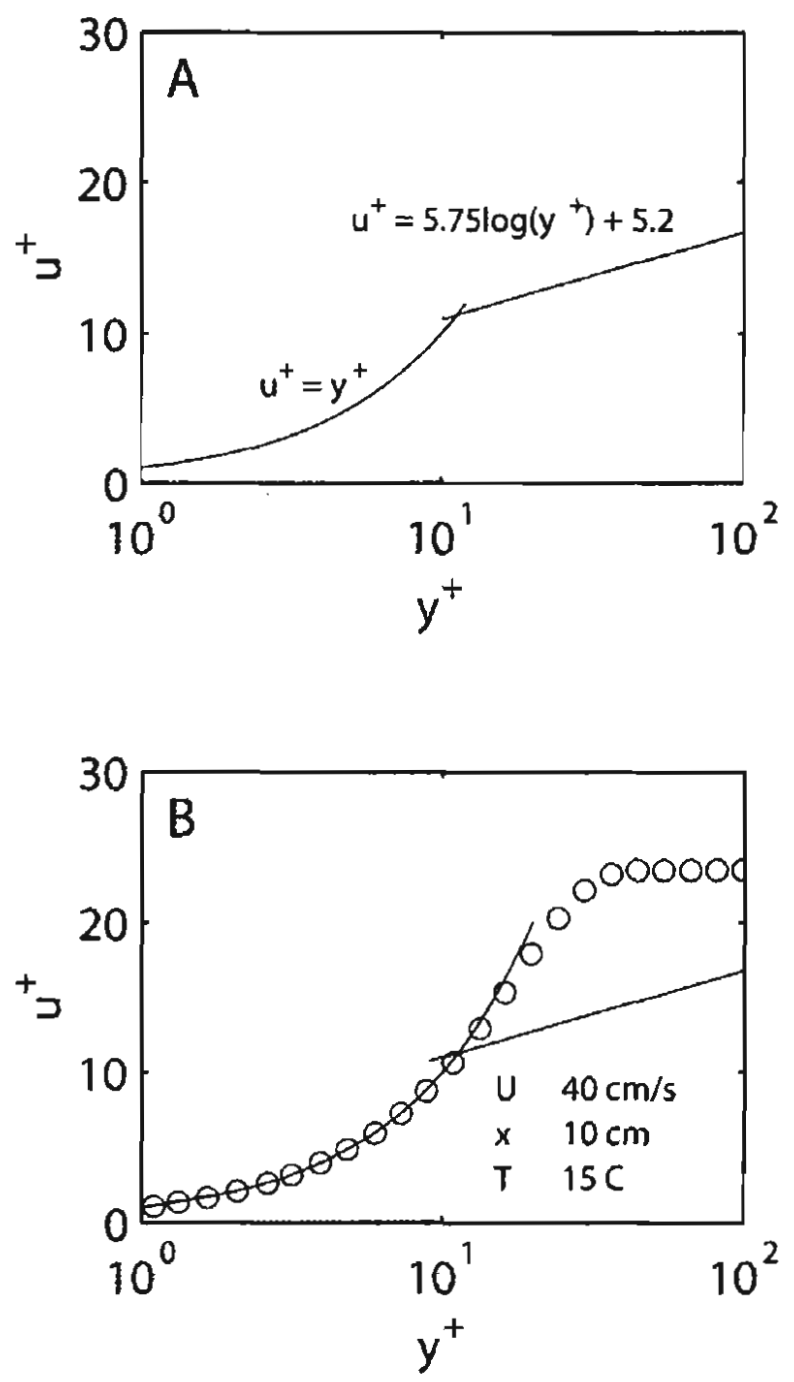

Fig. 2.1 Tangential boundary layer profiles presented as is conventional for the law of the wall. $u^{+}$and $y^{+}$are non-dimensionalized tangential velocity and normal distance from the body surface. (A) The time-averaged profile of the law of the wall for turbulent boundary layer flow over a flat plate with no streamwise pressure gradient plotted in non-dimensional wall units on a semi-logarithmic graph. (B) The tangential velocity profile of the laminar, zero streamwise pressure gradient, flat plate Blasius boundary layer, ' 0 ', scaled as for the law of the wall. The values used for velocity, $U$, streamwise position, $x$, and temperature, $T$, are within the experimental ranges of the present work. 
by PIV from individual image pairs, at most, can be considered time averages over an effective sampling period of $T_{s}=\ell / U$, where $\ell$ is the streamwise dimension of the field of view and $U$ is the swimming speed. $T_{s}$ in the experiments reported here ranged from 0.01 $-0.1 \mathrm{~s}$, much shorter than traditional sampling periods. This leads to uncertainty in the designation of certain profiles as turbulent unless several boundary layers at the same swimming speed, body position and body phase are acquired. Nevertheless, several fish boundary layer profiles at high Reynolds numbers showed excellent agreement with the law of the wall. More importantly, in the neighborhood of a particular surface position, the shapes of $u$-profiles in the linear sublayer of a turbulent boundary layer are less variable than those in the logarithmic region. Therefore, measurements of wall shear stress based on the linear sublayer, are accurate regardless of proper characterization of the boundary layer as matching a known profile shape.

\subsection{The $1 / 7^{\text {th }}$ power turbulent boundary layer profile approximation}

It can be shown that an equation of the form

$$
u(y)=\beta_{0} y^{1 / 7}
$$

is a reasonably good approximation for the tangential profile of a turbulent boundary layer over a flat plate with no streamwise pressure gradient. The law of the wall is better overall, but the $1 / 7^{\text {th }}$ power profile allows for a set of simple equations regarding $\delta_{9 g}$ and $\tau_{o}$ to be written, as for Blasius,

$$
\begin{aligned}
& \delta_{99}=0.373 x R e_{x}^{-1 / 5} \\
& \tau_{0}=0.0290 \rho U^{2} R e_{x}^{-1 / 5}
\end{aligned}
$$


These equations are used frequently in this investigation for simple comparisons related to turbulent boundary layers.

\subsection{Turbulence intensity}

The intensity of turbulence in the freestream flow affects the boundary layer over an object. The definition of turbulence intensity in a flow starts with separating the flow into the sum of a mean flow an each position $r$ in the flow, i.e. $U=U(r)$, and a fluctuating component, $u^{\prime}=u^{\prime}(r, t)$. Each component of the velocity, $U, V$ and $W$, can be similarly treated. Turbulence intensity for the $x$-direction is defined as

$$
I_{x} \equiv \frac{\sqrt{\frac{\sum_{i}^{n}\left[u^{\prime}\left(r, t_{i}\right)\right]^{2}}{n}}}{U_{o}}
$$

where each $t_{i}$ represents a time of sampling of the fluctuating velocity component $\mathrm{u}^{\prime}, n$ is the total number of samples, and $U_{0}$ is usually the overall mean freestream flow speed (Patton, 1984). This is simply the root-mean-square (RMS) of $u^{\prime}$ divided by the mean freestream flow. Overall turbulence intensity includes all three velocity components and is defined

$$
I \equiv \frac{\sqrt{\frac{\frac{1}{3} \sum_{i}^{n}\left(\left[u^{\prime}\left(r, t_{i}\right)\right]^{2}+\left[v^{\prime}\left(r, t_{i}\right)\right]^{2}+\left[w^{\prime}\left(r, t_{i}\right)\right]^{2}\right)}{n}}}{U_{o}}
$$

Turbulence intensity has a major impact on the value of the critical Reynolds number in boundary layer flow, i.e. the Reynolds number at which the boundary layer transitions to 
turbulence. Theory predicts a critical $R e_{x}$ of $\sim 2.5 \times 10^{6}$ for turbulence intensity close to 0 . As a rule of thumb however, a critical $R e_{x}$ of $3.5 \times 10^{5}$ to $5 \times 10^{5}$ is commonly reported for boundary layer transition. This range is that predicted for a freestream turbulence intensity of $1-2 \%$ by the theory of Van Driest and Blumer (1963). This is the turbulence intensity commonly found in good quality flumes.

\subsection{Boundary layer thickness}

As mentioned in the Introduction (section 1.1), since $u(y)$ in the boundary layer approaches the external flow velocity, $U_{e}$, asymptotically, boundary layer thickness is commonly defined as the height above the surface at which $u=0.99 U_{e}$. This quantity is given the symbol, $\delta_{9 g}$. In this thesis, another boundary layer thickness, $\delta_{95}$, defined at $u=$ $0.95 U_{e}$ is used. Standard deviation in flow velocities within the boundary layer was generally close to $1 \%$, thus automatic determination of the position $0.95 U_{e}$ was more robust. The Blasius boundary layer equation for $\delta_{95}$ is

$$
\delta_{95}=3.9 x R e_{x}^{-1 / 2} .
$$

Eqs. 2.3 and 2.13 show, that regardless of how it is defined, $\delta_{95}$ or $\delta_{99}$, boundary layer thickness grows as $x^{1 / 2}$. Eq. 2.10 shows that a turbulent boundary layer tends to grow faster, i.e. as $x^{4 / 5}$.

\subsection{Wall shear stress and friction drag}

Wall shear stress, and therefore skin friction, can be determined from tangential boundary layer velocity profiles. In the $u$-direction, the component of wall shear stress, $\tau_{o}$, is given by, 


$$
\tau_{o}=\left.\mu \frac{\partial u}{\partial y}\right|_{y=0}
$$

where $\mu$ is the dynamic viscosity of the fluid, $u=u(y)$ is the tangential component of fluid velocity over the object in the $x$-direction, and $y$ is in the direction of the local outward normal of the surface. In the linear sublayer of both laminar and turbulent boundary layers, the instantaneous value of the partial derivative-the normal gradient of $u$-at the body surface can be determined by a simple linear fit depending on the resolution of the flow. This use of experimental data to determine wall shear stress has been termed the 'near-wall method' by Österlund and Johansson (1999). Their wall shear stresses calculated from Eq. 2.14 using hot-wire velocity measurements show excellent agreement with theory and concurrent measurements of shear stress by the oil film technique (Siller, et al., 1993). They also determined and verified fluctuating shear stress measurements, due to the unsteadiness of turbulent flow, with MEMS-type hot films.

The wall shear stress distribution, $\tau_{o}$, over an object can be used to calculate the total friction drag, $D_{f}$, using,

$$
D_{f}=\iint_{S} \tau_{o} d A \cos \theta
$$

where $S$ is the three-dimensional function defining the body surface of the fish, $d A$ is the incremental area over which a particular shear stress applies, and $\theta$ is the angle between the body surface tangent in the laser plane and the streamwise direction. The coefficient of friction for any object is defined as,

$$
C_{f}=\frac{D_{f}}{\frac{1}{2} \rho A U^{2}}
$$


where $\rho$ is the fluid density, $A$ is the total wetted surface area of the body, and $U$ is the relative velocity of the object through the fluid. In order to obtain accurate values of friction drag and the coefficient of friction for a swimming fish, a large number of measurements of wall shear stress at different positions and at different phases of the undulatory motion must be taken.

For comparison purposes, a local coefficient of friction, $C_{f x}$, was defined as,

$$
C_{f x} \equiv \frac{\tau_{o}(x)}{\frac{1}{2} \rho U^{2}}
$$

By this definition, $C_{f}$ is the area average of $C_{f x}$ over the fish surface. Therefore, $C_{f}$ for a given fish falls between the maximum and minimum values of $C_{f x}$ determined over the fish body. Both time averaged and instantaneous values of $C_{f x}$ were examined. 
Page 38 


\section{Chapter 3}

\section{Preliminary investigation}

This chapter is an abridged version of the Methods and Materials, Results and Discussion from the paper titled 'The boundary layer of swimming fish' published in the Journal of Experimental Biology by the author, W. R. McGillis, and M. A. Grosenbaugh (Anderson et al., 2001a). The paper describes the successful visualization of the fish boundary layer by a highly manual data acquisition and analysis system.

\subsection{Methods and materials}

\subsubsection{Fish}

Scup, Stenotomus chrysops, $(n=9)$ and smooth dogfish, Mustelus canis, $(n=1)$, were caught in traps or by hook and line in Nantucket Sound, off Woods Hole, MA, USA. The animals were kept in 750-liter tanks with a constant flow of fresh seawater from Nantucket Sound. All fish kept longer than 2 days were fed a steady diet of frozen squid. Fish were transferred to and from their tanks in 30-liter buckets or 60-liter coolers. Following experiments, fish were euthanized by cervical transection according to the WHOI Institutional Animal Care and Use Committee (IACUC) protocol at the time of the experiments. The body length, $L$, of scup averaged $19.5 \pm 1.8 \mathrm{~cm}$ (mean $\pm S . D$.). The dogfish measured $44.4 \mathrm{~cm}$.

\subsubsection{Swimming conditions}

Scup were observed swimming in both still water and in a flume. In still water, scup were observed swimming $3-40 \mathrm{~cm} \mathrm{~s}^{-1}$ at water temperatures of $11 \mathrm{C}$ or $22-25 \mathrm{C}$, 
depending on the season during which the experiments were run. In the flume, scup were observed swimming $10-65 \mathrm{~cm} \mathrm{~s}^{-1}$ at $22-23 \mathrm{C}$. The dogfish was observed swimming 20 $-65 \mathrm{~cm} \mathrm{~s}^{-1}$ in the flume at $22-23 \mathrm{C}$.

In flume trials, observations from three positions along the midline of each fish were performed at one or more speeds. In scup, the measurements were made at $x=$ $0.50 \mathrm{~L}, 0.77 \mathrm{~L}$, and $0.91 \mathrm{~L}$. In dogfish, the measurements were made at $x=0.44 \mathrm{~L}, 0.53 \mathrm{~L}$, and $0.69 \mathrm{~L}$. The majority of flume data for scup was acquired at swimming speed $30 \mathrm{~cm}$ $\mathrm{s}^{-1}$ (18 swimming sequences). At this speed, scup were observed to use primarily caudal fin propulsion with infrequent strokes by their pectoral fins. Records of transverse velocity showed continuous undulatory swimming during all acquired sequences. In still water, scup tended to swim more slowly, frequently using their pectoral fins and gliding. Therefore, in our analysis of the fish boundary layer, we have concentrated on the flume experiments and the fastest of the still water swimming sequences. The majority of the flume data for the dogfish was acquired at the swimming speed $20 \mathrm{~cm} \mathrm{~s}^{-1}$ (22 swimming sequences). Rigid-body measurements in dogfish were made at two positions, $x=0.44 \mathrm{~L}$ and $0.69 \mathrm{~L}$ at $20 \mathrm{~cm} \mathrm{~s}^{-1}$. The more forward positions on the dogfish were chosen because it was difficult to acquire sufficient data in the posterior region where the body wave amplitude increases dramatically with position. At positions posterior to $x \approx 0.75 \mathrm{~L}$, the fish surface was captured infrequently in the small field of view of the flow-imaging camera. The swimming speeds of $30 \mathrm{~cm} \mathrm{~s}^{-1}$ in scup and $20 \mathrm{~cm} \mathrm{~s}^{-1}$ in dogfish were chosen because at these speeds the fish swam steadily for long periods of time without tiring.

Still water trials were performed in a large rectangular tank $(2.5 \mathrm{~m} \times 1.2 \mathrm{~m} \times 0.5$ m). Water depth was $20 \mathrm{~cm}$. A channel, $20 \mathrm{~cm}$ wide, was constructed along one of the long glass walls of the tank. The midpoint of the channel was used as the test section. The flow-imaging camera was partially submerged in a glass enclosure to prevent free surface optical distortion. Fish swam deeply and slowly enough so that free surface wave effects were negligible. Flowing water trials were performed in a large, recirculating, 
open-channel flume capable of speeds up to $70 \mathrm{~cm} \mathrm{~s}^{-1}$. The racing oval shaped flume, with straight-aways $7.6 \mathrm{~m}$ long, is paddle driven by a conveyor belt mechanism. The flume channel is $78 \mathrm{~cm}$ wide and $30 \mathrm{~cm}$ deep. Water depth during fish swimming trials was $16 \mathrm{~cm}$. The test section used was constructed against one of the glass walls of the flume, $20 \mathrm{~cm}$ wide and $80 \mathrm{~cm}$ long. The free surface was eliminated using a sheet of acrylic. Honeycomb flow-through barriers bound the test section, confining the fish to the test section, and damping out large-scale flow disturbances. The barriers were 12.7 $\mathrm{cm}$ in streamwise length with tube diameter of $1.3 \mathrm{~cm}$. Turbulence intensity in the test section measured by laser Doppler anemometry, LDA, was 4 - $6 \%$ over the range of experimental flow speeds. Without the honeycomb barriers, turbulence intensity measured 7-8\%. Velocity measurements outside of the fish boundary layer demonstrated scatter in agreement with the measured test section turbulence intensity. Still water trials showed little to no scatter in velocity outside the boundary layer. In both still and flowing water trials, fish swam far enough from the wall on the side of the fish measured-generally 8 - $12 \mathrm{~cm}$--that wall effects are expected to be minimal.

\subsubsection{Image acquisition}

Fluid flow around the fish was illuminated by a horizontal laser sheet, $0.5 \mathrm{~mm}$ thick, and imaged from above with a high-resolution digital video camera (Kodak ES 1.0, 1008 pixels x 1018 pixels) - the 'boundary layer camera' shown in Fig. 3.1. The second camera shown above the test section in Fig. 3.1, the 'nearfield camera' was added in the advanced study (see Chapter 4). The flow was seeded with neutrally buoyant fluorescent particles, 20 - $40 \mu \mathrm{m}$ in diameter. Macro photographic lenses (Nikon, Micro-Nikkor, $60 \mathrm{~mm}$ ) were used to obtain high quality, high magnification images of particles in the flow over the fish surface (Fig. 3.2). Fields of view used with the particle imaging camera were $1-2 \mathrm{~cm}$ on a side. The resulting images had a scale of $50-100$ pixels $\mathrm{mm}^{-1}$. Our fish boundary layers measured $0.5-12 \mathrm{~mm}$ in thickness. The laser (New Wave Research, Nd:YAG, dual pulsed) was operated at low power to prevent irritation to 


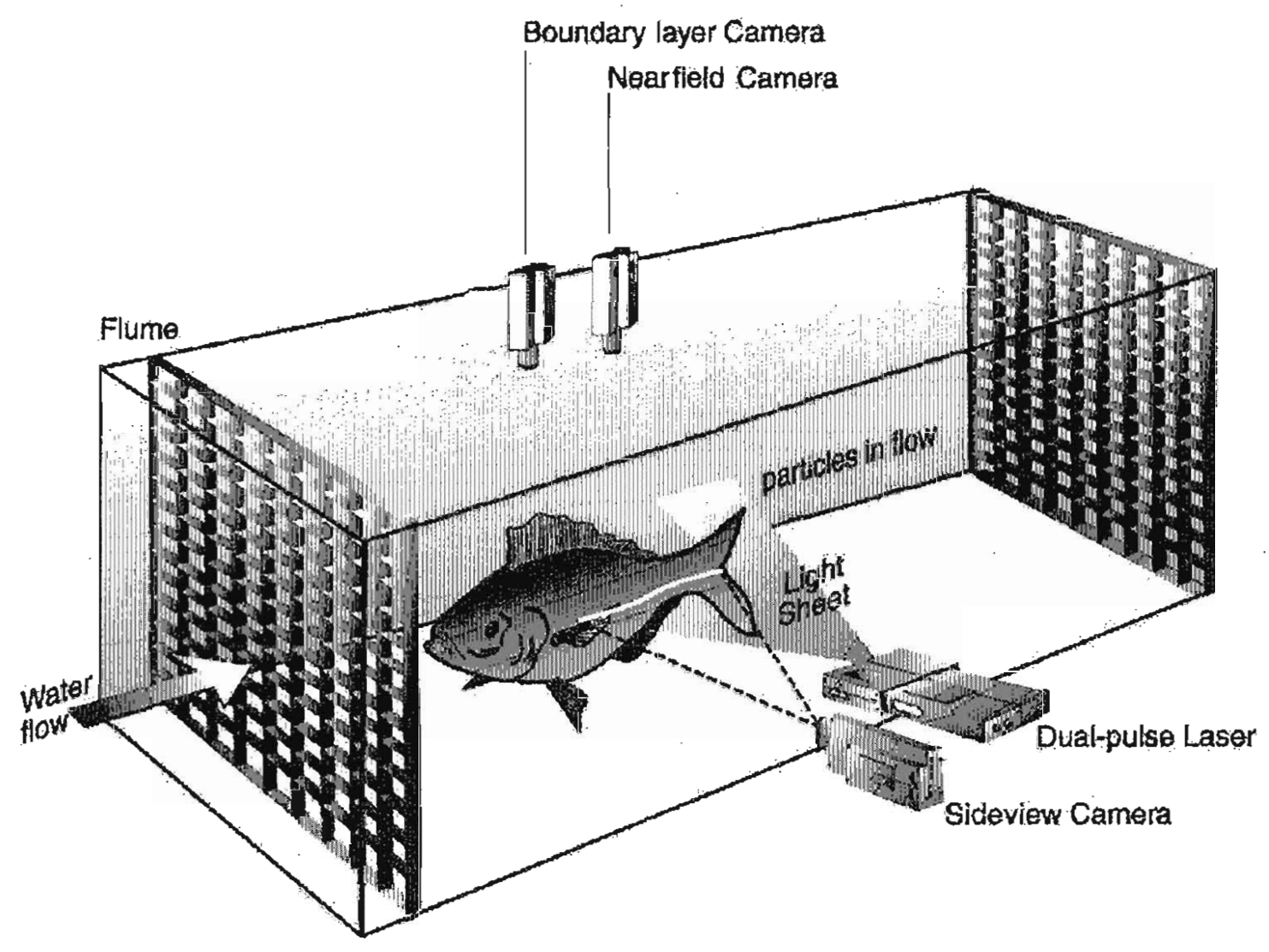

Fig. 3.1 Sketch of the setup for boundary layer visualization. The bright line on the fish centerline shows where the laser impinges on the fish surface. The nearfield camera was added after the preliminary investigation. The boundary layer and nearfield cameras were also moved underneath the test section. See text for information about the variety of barriers used to constrain the fish to the test section. 


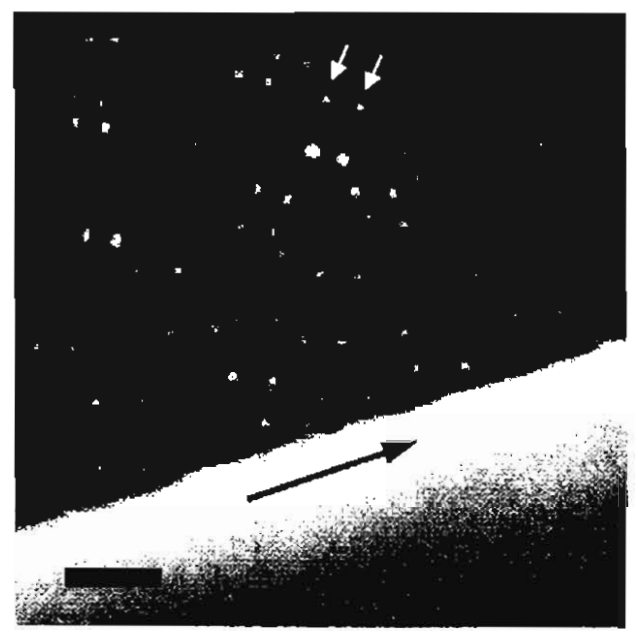

Fig. 3.2 A double exposure showing examples of particle pairs used to determine fluid velocities in the boundary layer around a swimming scup. A particle pair is labeled with white arrows. The particles in the image were moving roughly left to right. Scale bar is $1 \mathrm{~mm}$. The camera angle was as shown in Fig. 3.1 (the boundary layer camera). The body surface of the scup appears as a sharp, bright edge in the lower half of the image. The position on the scup shown is $x=0.55 \mathrm{~L}$ on the midline of the fish. The scup was swimming $8.3 \mathrm{~cm} \mathrm{~s}^{-1}$ through still water, roughly to the right in the field of view (black arrow). The body surface was moving laterally $1.7 \mathrm{~cm} \mathrm{~s}^{-1}$ in the direction away from the region of fluid shown here in the upper portion of the image. Note that the particles closer to the fish move a greater distance than particles further away from the fish. This is because the fluid closest to the fish is influenced most by the motion of the fish through the fluid. However, in the frame of reference of the fish, the particles closest to the fish are moving more slowly than the particles further from the fish, resulting in boundary layer profiles similar to those shown in Fig. 1.1. The double exposure was constructed simply by adding successive video images. The image was swept of approximately half of its original particles and threshold filtered for clarity of presentation. 
the animal and to minimize glare. The time delay, $\Delta t$, between laser pulses, i.e. between exposures of the flow, was set at 2 - 10 ms depending on swimming speed. The measured displacement of particles between exposures is divided by this time to obtain particle velocities. The laser and the particle imaging camera were synchronized using a digital delay triggered by every other vertical drive signal of the camera. The vertical drive signal is a TTL pulse that signals the moment between two exposures. When triggered, the digital delay triggered laser 1 of the dual laser to fire $\Delta t / 2$ before, and laser 2 to fire $\Delta t / 2$ after, the next vertical drive signal of the camera, which was 'ignored' by the digital delay. The camera was operated at approximately $30 \mathrm{~Hz}$ and 100 sequential images were acquired per swimming sequence. Therefore, pairs of exposures, or image pairs, were acquired at $15 \mathrm{~Hz}$, and continuous sequences of 50 pairs were acquired. Two standard video cameras were used to obtain simultaneous records of whole body motion in lateral and dorsal views. This allowed fish boundary layer flow to be compared with relevant instantaneous whole body kinematic parameters.

Measurements were confined to positions on the fish where the body surface was essentially perpendicular to the laser sheet. As the angle between the laser sheet and the fish surface deviates from $90^{\circ}$, boundary layer velocity profiles are distorted, tending to give an incorrectly low wall shear stress. Images in which the fish surface is perpendicular to the laser sheet are easily distinguished from images in which the surface is at an angle to the sheet. In the former, the fish surface appears as a sharp edge. In the latter, depending on the direction of tilt, either the intersection of the beam and the fish surface is not visible, or the features of the fish surface beneath the sheet are visible, dimly illuminated by reflected laser light. Only images of the former type were used in the analysis.

In both still water and flume trials, all three video cameras were fixed with respect to the frame of the test section during image acquisition. In still water, the fish swam through the test section. Therefore they swam through each camera's field of view at 
their swimming speed, $U$, and flow velocity outside the fish boundary layer was nearly zero. In the flume, fish held station in the test section without significant streamwise motion with respect to the fields of view. The flow outside the boundary layer of the fish therefore moved through the fields of view at the approximate flume speed, $U$. Apart from the ambient turbulence of the flume flow, the two situations are equivalent from the standpoint of fluid dynamics. Both techniques proved useful to the analysis of the fish boundary layer. Still water trials revealed actual boundary layer development over particular fish in undisturbed flow, whereas flume trials revealed the phase dependent aspects of the boundary layer at selected positions on the fish. The flume was also used to look at boundary layer development by recording several sequences from various streamwise positions.

\subsubsection{Rigid-body drag}

In general, the dogfish swam very close to the bottom of the flume, and it was possible to measure the boundary layer of the dogfish at the same streamwise position and flume speed for both swimming and resting. Three image sequences of the dogfish boundary layer were acquired while the dogfish conveniently rested motionless on the bottom of the flume. The flume speed and water temperature were $20 \mathrm{~cm} \mathrm{~s}^{-1}$ and $23^{\mathrm{c}}$. The resting data were used to determine rigid-body friction drag for the dogfish.

It was important to confirm that the bottom boundary layer of the test section did not affect the rigid-body measurements significantly. LDA showed that the boundary layer of the test section bottom was thinner than $1.5 \mathrm{~cm}$. Dogfish boundary layer data was taken between $1.2-1.8 \mathrm{~cm}$. Flow visualizations were therefore made outside, or at the outer edge of the flume bottom boundary layer, where small changes in the height would not be expected to have a significant effect on the flow velocities at the outer edge of the boundary layer, $U_{e}$. Velocities measured by particle tracking confirmed this. $U_{e}$ in 
both the swimming and rigid-body cases was found to be essentially the same at $x=$ $0.44 L$.

\subsubsection{Digital particle tracking velocimetry}

The acquisition and analysis of image pairs for digital particle imaging velocimetry, DPIV, and digital particle tracking velocimetry, DPTV, is now common practice among engineers, chemists and a growing number of biologists. For this reason the details of these techniques will be left to the numerous existing works on the subject; the reader is referred to Adrian (1991), Willert and Gharib (1991) and Stamhuis and Videler (1995). Here, we report the variations on the themes of DPIV and DPTV necessary to capture and resolve the fish boundary layer. Flow velocities around the fish were quantified primarily by semi-automatic DPTV (Stamhuis and Videler, 1995). Particle pairs are located manually with a cursor on the computer screen. The term 'particle pair' refers to the two images of the same particle that occur in an image pair. A

particular image pair typically has tens to hundreds of particle pairs depending on seeding density. Once the particle pairs have been located, a computer program then determines the centroids of the particles and calculates displacement and velocity. Conventional DPIV and automatic particle tracking code were sometimes used to resolve the outermost regions of boundary layer flow, but they often failed to resolve the flow very close to the moving surface of the fish.

The fish surface was located using an edge detection algorithm developed in the study of squid locomotion (Anderson and DeMont, 2000; Anderson et al., 2001b). The algorithm was further developed in the course of the present work to match surface features in sequential images and thereby calculate the precise motions of the animal surface. This motion was conveniently described by a tangential and normal displacement. Deformation and rotation of the fish surface was found to be negligible for any image pair due to the short time separating the images and the small field of view. 
Trials during which the fish rested motionless on the bottom of the tank revealed the accuracy of this wall-tracking algorithm to be better than 0.5 pixels. At our magnifications, this represents $10-20 \mu \mathrm{m}$ error in displacement and, after smoothing, negligible error in surface slope. For a typical swimming trial, say $U=20 \mathrm{~cm} \mathrm{~s}^{-1}$ and $\Delta t$ $=5 \mathrm{~ms}$, this translates to less than $2 \%$ error in the measurement of tangential flow velocity relative to the fish surface. Average maximum error in normal velocity is 2 $10 \%$, depending on the magnitude of the transverse body velocity. Since wall shear stresses were determined from the slope of the boundary layer profile near the body surface, such errors in velocity relative to the fish surface do not affect our calculated skin friction. Instead, these errors impact less critical measurements, such as outer edge velocity, boundary layer thickness, and their fluctuations. In general, these parameters were large enough that errors were insignificant to negligible.

\subsubsection{Tangential and normal velocity calculations}

To construct tangential and normal velocity profiles from the image pairs of flow over the fish surface, the motion of particles in the image pairs must be viewed from the reference frame of the fish. Unless the surface can be described by a straight line, this requires the construction of axes normal and tangential to the fish surface for each particle. Assuming the velocity profiles do not change significantly over the relatively small field of view, this method results in the desired boundary layer profiles. The separate profiles are built up from the normal and tangential components of velocity determined for each particle, with respect to the fish, plotted against normal distance of the particle from the fish surface.

Normals from particles to the fish surface were determined though a standard minimization of the distances from the particles to the fish surface. The radius of curvature of the fish surface was always larger in scale than the field of view. This ensured convergence of the minimization process. The fish body surface was found to be 
well fit with a cubic polynomial. This was used as a means to smooth surface roughness, reducing needless scatter in the minimization process. The normal velocity, $v$, of a particle with respect to the fish was calculated by,

$$
v=\frac{y_{2}-y_{1}}{\Delta t}
$$

where $\Delta t$ is the time between laser pulses, and $y_{1}$ and $y_{2}$ are the lengths of the normals for the particle in the first and second images respectively. This simple equation can be used because, as mentioned earlier, the deformation and rotation of the fish surface was negligible over the time between images, $\Delta t$.

The calculation of tangential velocity also began by determining normals to the fish surface from points in the fluid by the same distance minimization. In this case, however, the normals were determined from the midpoint of a particle track to the average position of the fish surface in the two images. The slope of the average fish surface was determined at the intersection of the normal and the average fish surface. The slope was used to construct a unit tangent vector, $t$, of the average fish surface, in a streamwise sense, with respect to the camera pixel coordinates. That is, the vector lies in the horizontal plane of the laser sheet, is tangent to the fish surface and points roughly in the caudal direction. The velocity of the particle, $V_{p}$, and the velocity of the fish surface, $V_{s}$, were determined in the same coordinate system. The tangential velocity, $u$, of the particle with respect to the fish was then determined by the vector operation,

$$
u=\left(V_{p}-V_{s}\right) \cdot t
$$

that is, $u$ is the component of the velocity of the particle, relative to the fish surface, in the direction of the surface unit tangent vector in the plane of the laser sheet. Therefore, $u=$ 0 at the fish surface and $u=U_{e}$ at the edge of the boundary layer. The normal velocity of 
the particle with respect to the fish can be determined in a similar manner, but normal velocities calculated from Eq. 3.1 are more accurate since fish surface averaging is sidestepped. In some instances, conventional DPIV was used to resolve the outer boundary layer and nearfield, reducing the tedium of semi-automatic DPTV processing. The better the seeding, the closer to the fish DPIV could be used with confidence. DPIV nodes were treated as the positions of virtual particles in the first image, and the locations of correlation peaks were treated as virtual particle positions in the second image. This use of DPIV was made only well beyond the linear sublayer of the boundary layer and only when particle densities allowed. The linear sublayer is the region of the boundary layer closest to the body surface in which the tangential velocity profile is linear. It will be shown later that an accurate determination of velocities in the linear sublayer is critical to the analysis of skin friction. As expected in instances of proper seeding, cross checks of such DPIV data by DPTV showed negligible differences in velocities calculated in outer regions of the boundary layer.

\subsubsection{DPTV errors}

Absolute errors in DPTV depend on camera pixel resolution, field of view dimensions, particle shape, size, centroid analysis, and image quality. Relative errors are magnified by decreased particle displacements, which depend on $\Delta t$ and the field of view dimensions. We estimate average maximum DPTV errors of tangential velocities in the linear sublayer of the fish boundary layers to be between $5-15 \%$. This range arises from conservative estimates of sub-pixel accuracy and particle displacements on the order of 10 pixels. These errors tend to be unbiased since they depend on the images of individual particles. Therefore, if enough particle pairs are sampled in a given image pair, the error in wall shear stress determined for that image pair tends to be unbiased. Wall shear stress is determined from a linear fit of the $u$-profile in the linear sublayer. 
Increased scatter was commonly observed in our $v$-profile data compared to the $u$ profile data. This is probably due to DPTV errors magnified by generally shorter normal displacements. Turbulence, wall tracking errors, variation in the profile over the streamwise length of the field of view and cross-stream surface curvature may also contribute to scatter in our profiles. In still water, very little scatter was observed in our $u$-profiles, especially outside the boundary layer, where particles are nearly stationary in the field of view. This is strong support for setting our DPTV error toward the lower end of our estimated $5-15 \%$ mentioned earlier.

\subsubsection{Undulatory phase}

Boundary layer data were taken on one side of the fish for any given trial. The fish surface oscillated in the field of view of the particle imaging camera due to transverse motion of the body. We will use the term 'crest' to describe the instance when the section of the fish surface in view has moved to its full amplitude in the direction of the outward pointing surface normal, that is, the positive $y$-direction. We use 'trough' for the instance of full amplitude in the negative $y$-direction. Phase is set to $90^{\circ}$ at crest and $270^{\circ}$ at trough. Transverse wall velocity as a function of time determined from wall tracking was fit with a sine function. The phase of the body surface transverse position was determined by integrating wall velocity, or simply subtracting $90^{\circ}$ from the phase of transverse wall velocity.

Detailed phase analysis was only applied to flume data. Still water trials result in a more complicated mix of phase and position. The propulsive wave of the fish travels streamwise at a speed slightly greater than the swimming speed, $U$ (Gray, 1968). Since, for still water, the field of view is fixed with respect to the bulk fluid in the tank, phase appears to change more slowly than if observed in a flume. If the wave speed were nearly equal to the swimming speed almost no change in phase would be observed. 
Therefore, still water trials give information at various phases at various positions. In contrast, flume trials give information at one position as a function of phase.

\subsection{Results}

\subsubsection{Fish boundary layer profiles}

More than 70 swimming sequences of scup and 30 sequences of dogfish were acquired, yielding hundreds of usable image pairs for boundary layer realization. Tangential and normal velocity profiles were determined for more than 270 image pairs from 36 swimming sequences with high image quality over the full range of experimental speeds. Only one dogfish has so far been examined and so generalizations concerning anguilliform swimmers must be considered tentative. Nevertheless, the quantity and consistency of the dogfish data suggest that the conclusions regarding the specimen observed are well founded.

Fish boundary layer profiles tended to resemble the solutions of either Blasius or the law of the wall (Fig. 3.3). Profiles that deviated from these two types often exhibited good agreement with the Falkner-Skan solution (Fig. 3.4). The Falkner-Skan solution can describe either an accelerating (Fig. 3.4A,B) or a decelerating (Fig. 3.4C,D) boundary layer depending on the choice of the coefficient, $m$, in the Falkner-Skan differential equations (see section 2.3). Boundary layers are classified as accelerating or decelerating on the basis of their $u$-profiles. However, in the instantaneous profiles of a boundary layer, the evidence of acceleration or deceleration is found in the v-profile. Negative normal velocity at the outer edge of the boundary layer (Fig. 3.4B) reveals that there is a net normal flow of fluid, or normal flux, into the boundary layer characteristic of an accelerating boundary layer. In contrast, the Blasius solution always shows positive normal velocity at the edge of the boundary layer (Figs. 3.3B,3.4B), and is therefore a decelerating boundary layer. 

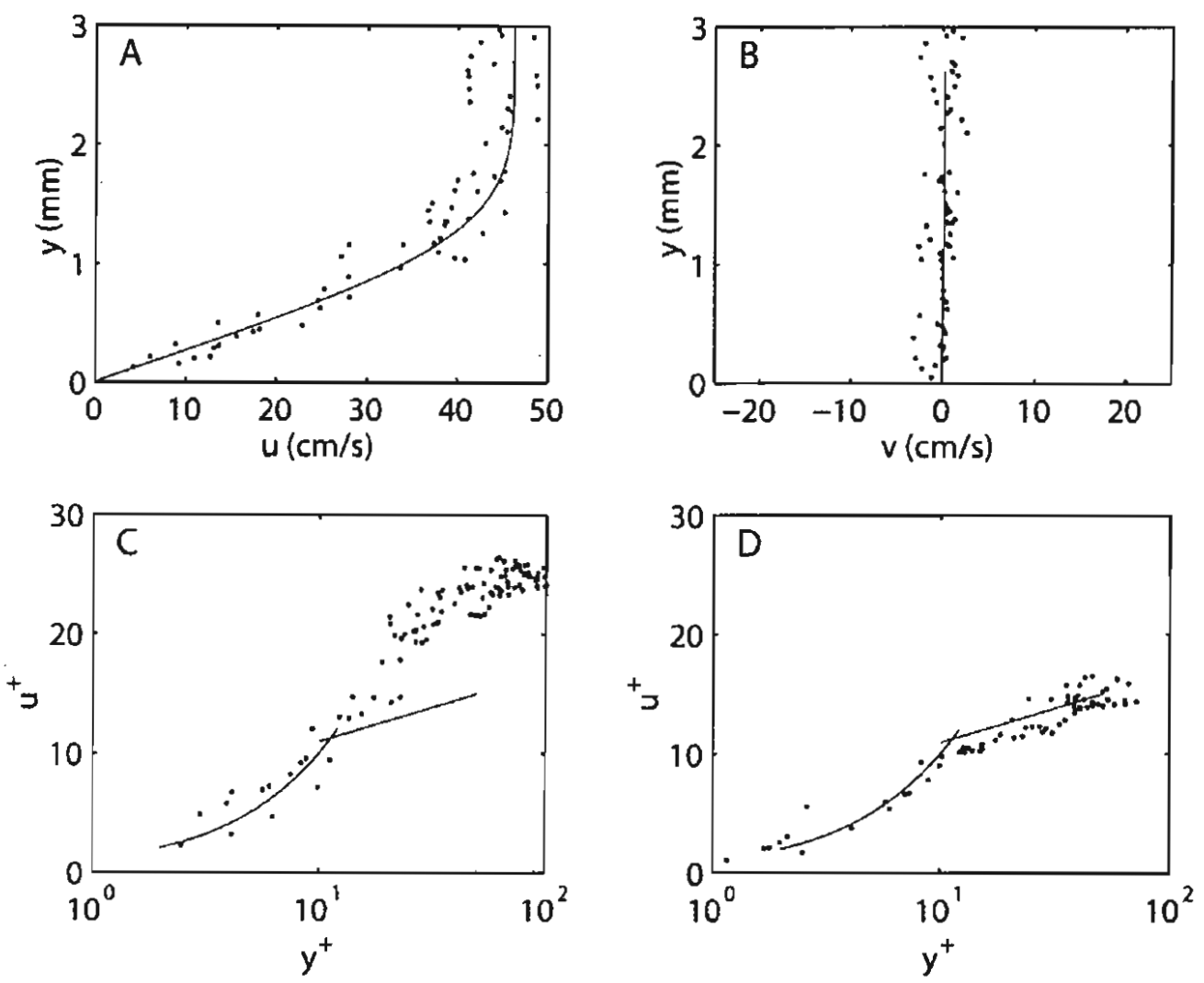

Fig. 3.3 Two representative boundary layer realizations illustrating the distinction between laminar-like and turbulent-like boundary layers.

Each data point represents information calculated from one particle pair of the image pairs used for the given realizations. The first realization shown (A-C) is from $x=0.50 L$ on a scup swimming in the flume at 42 $\mathrm{cm} \mathrm{s}^{-1}, R e_{x} \cong 4 \times 10^{4}$. The second (D) is from $x=0.53 \mathrm{~L}$ on the dogfish swimming in the flume at $20 \mathrm{~cm} \mathrm{~s}^{-1}, R e_{x} \cong 4 \times 10^{4}$. (A) The $u$-profile of the first realization showing agreement with a Blasius fit drawn as a solid curve. (B) The v-profiles of the first realization and the Blasius fit of (A). (C) The $u$-profile of the first realization compared to the law of the wall by fitting the linear sublayer. The boundary layer distinguishes itself as laminar-like as outlined in Fig. 2.1. (D) The dogfish boundary layer realization showing good agreement with the law of the wall, distinguishing the profile as turbulent-like. Note the slight shift in the logarithmic region. The fit exhibits sharp contrast to the fit of the profile shown in (C). 

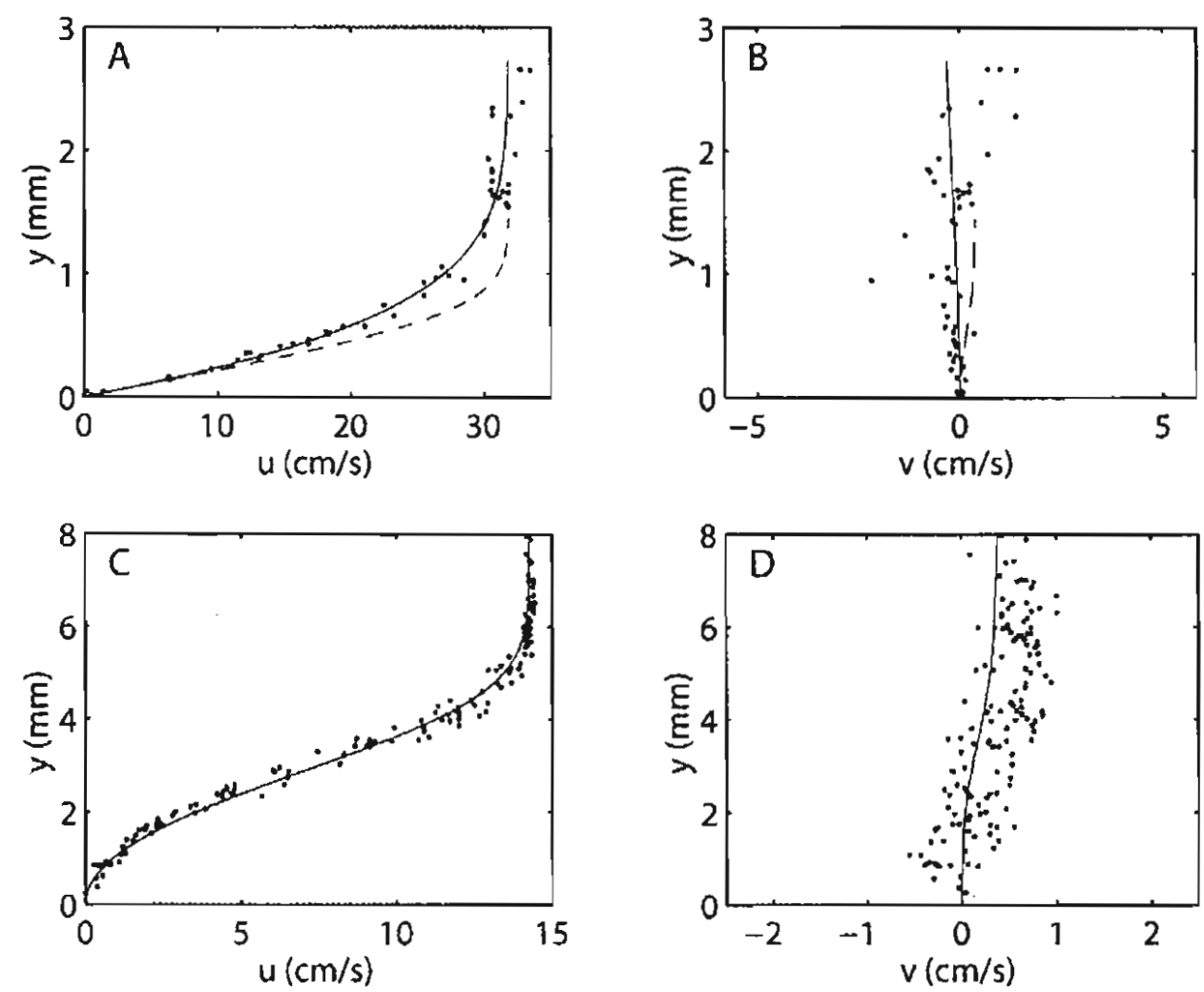

Fig. 3.4 Two representative boundary layer realizations that are fit well by the Falkner-Skan solution. The first realization shown $(\mathbf{A}, \mathbf{B})$ is from $x=0.50 \mathrm{~L}$ on a scup swimming in the flume at $30 \mathrm{~cm} \mathrm{~s}^{-1}, R e_{x} \cong 3 \times 10^{4}$. The second (C,D) comes from very close to the body trailing edge of a scup swimming in still water at $14 \mathrm{~cm} \mathrm{~s}^{-1}$ and decelerating at $10 \mathrm{~cm} \mathrm{~s}^{-2}$, $R e_{x} \cong 2 \times 10^{4}$. (A) The $u$-profile of the first realization with a FalknerSkan fit drawn as a solid curve. The dashed curve is the Blasius solution with the same wall shear stress. (B) The v-profiles of the first realization, the Falkner-Skan solution and the Blasius solution. (C) The $u$-profile of the second realization. (D) The v-profile of the second realization. The solid curve is the Falkner-Skan fit. 
The connection between normal flux and acceleration has to do with the incompressibility and continuity of water. Imagine a constant diameter pipe carrying water with a prescribed upstream volume input. If the pipe is tapped, so that water can be pumped in or out, the downstream volume flow of the pipe can be changed.

Incompressibility and continuity require that the flow speed must also change. If water is pumped in, flow must accelerate in the pipe in the vicinity of the tap. If we pump water out, the pipe flow decelerates.

Fish boundary layer profiles occasionally resembled strongly decelerating Falkner-Skan profiles characterized by highly inflected $u$-profiles with low wall shear stress (Fig. 3.4C). The v-profiles of these realizations revealed flow out of the boundary layer characteristic of boundary layer deceleration (Fig. 3.4D). Inflected boundary layers of this type are often a sign of incipient separation (Batchelor, 1967). No profiles indicative of separation were observed.

\subsubsection{Flow condition in the boundary layer}

In still water trials, boundary layer profile shapes always suggested laminar flow. This is not entirely surprising since Reynolds numbers, $R e$, were $3 \times 10^{3}$ to $6 \times 10^{4}$, lower than the standard critical range for boundary layer transition, $R e_{x}=3.5 \times 10^{5}-5 \times 10^{5}$. This range represents the predicted transition $R e_{x}$ for a flat plate in a flow exhibiting a turbulence intensity of 1 - 2\% (Van Driest and Blumer, 1963). In flume trials, however, both laminar and turbulent profile shapes were observed even though Reynolds numbers did not quite reach the critical value. The ambient turbulence of the flume, the roughness of the fish surface and the unsteadiness of the flow over the fish might be expected to trip turbulence at lower Reynolds numbers. The boundary layer over scup swimming in the flume at $30 \mathrm{~cm} \mathrm{~s}^{-1}, R e=6 \times 10^{4}$, was apparently al ways laminar over the entire body. The boundary layer over a dogfish swimming $63 \mathrm{~cm} \mathrm{~s}^{-1}, R e=3 \times 10^{5}$, measured at $x=$ 
$0.63 L, R e_{x}=1.9 \times 10^{5}$, appeared to be primarily turbulent. In some cases, at Reynolds numbers between these two values, the boundary layer apparently oscillated between laminar and turbulent. When this was observed, turbulent profile shapes tended to appear at the crest phase of the body wave. The boundary layer would generally return to a laminar shape during the crest to trough motion.

The rigid-body case of the dogfish revealed an interesting effect. Flow appeared laminar at $x=0.44 L$ and turbulent at $x=0.69 \mathrm{~L}$. For the swimming dogfish, boundary layer flow appeared to be laminar at $x=0.44 \mathrm{~L}$ and $x=0.69 \mathrm{~L}$ for most of the time with some evidence of oscillating between laminar and turbulent at $x=0.69 \mathrm{~L}$. The observation of laminar boundary layer flow at $x=0.69 \mathrm{~L}$ during swimming suggests a stabilization process. The same phenomenon was observed by Taneda and Tomonari (1974) comparing the boundary layer flow for the rigid-body and various swimming cases of a waving plate.

\subsubsection{Local friction coefficients}

Posterior to $x=0.8 L$ in scup and $x=0.5 L$ in dogfish, the time averaged local friction coefficients, $C_{f x}$, of both species increase above the flat plate laminar and turbulent values (Figs. 3.5,3.6). This increase in friction is much more dramatic in the anguilliform swimmer. Local friction coefficients in the rigid-body case of the dogfish do not show this increase and remain in between the laminar and turbulent flat-plate values, i.e. the friction drag on the swimming dogfish is higher than that on the dogfish stretched straight in the flow.

In many cases, the values of $C_{f x}, U_{\mathfrak{t}}$, and $\delta$ vs. relative position, $x / L$, were observed to depend both on species and the sign of the transverse velocity of the fish surface (Fig. 3.6). $C_{f x}$ increases out of the range of flat plate friction more forward on the body of the dogfish than on the scup (Fig. 3.6A,B). In both species, local friction 


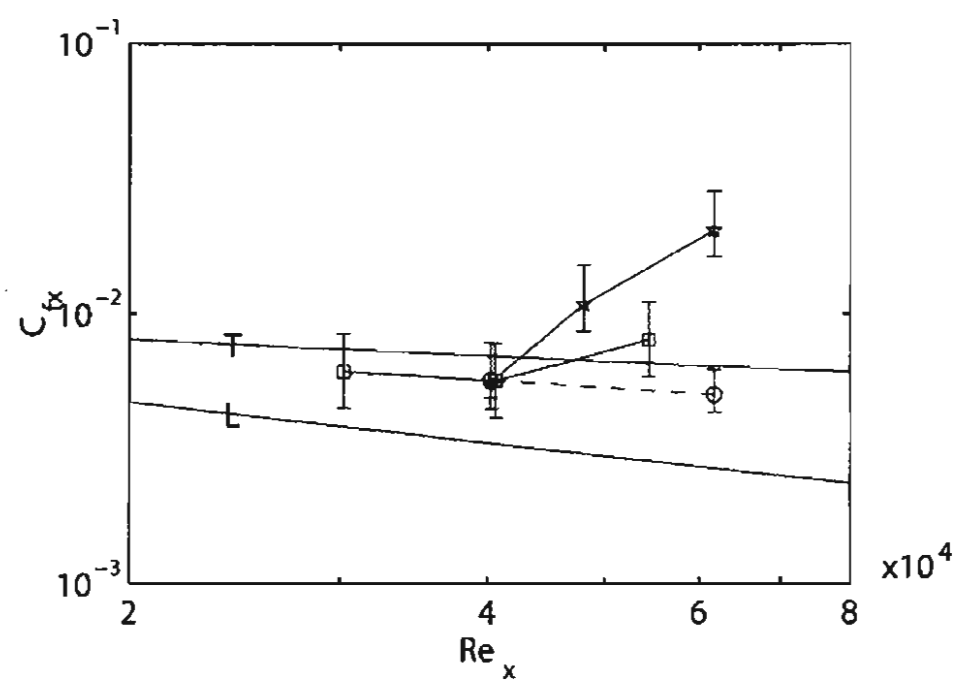

Fig. 3.5 Time averaged local friction coefficients, $C_{f x}$, vs. local Reynolds number, $R e_{x}$, on scup, ' $\square$ ' and dogfish, ' $\hbar$ ', including the rigid body case the dogfish, 'o.' $C_{f x}$ is plotted versus $R e_{x}$ because it is known that geometrically similar objects all have the same distribution of $C_{f x}$ with $R e_{x}$ regardless of size, speed or fluid environment. Plotting the local friction coefficient vs. $R e_{x}$ is therefore the best way to compare the distribution of friction over a set of objects in varying conditions of size, speed and viscosity. The data were averaged over several locomotory cycles from several swimming sequences at the same flume speed for each species at $22-23$ C: $U=20 \mathrm{~cm} \mathrm{~s}^{-1}$ for the dogfish, $U=30 \mathrm{~cm} \mathrm{~s}^{-1}$ for scup. The lines labeled ' $T$ ' and ' $L$ ' are flat plate friction for turbulent and laminar boundary layer flow with no streamwise pressure gradient. On average, each data point shown, representing a whole cycle average, represents 8 boundary layer realizations for scup, 34 realizations for the swimming dogfish and 8 realizations for the rigid dogfish. Error bars are based on the maximum percent errors in the determination of the slope of the linear sublayer, i.e. the wall shear stress, for the boundary layer profiles contributing to each data point. 

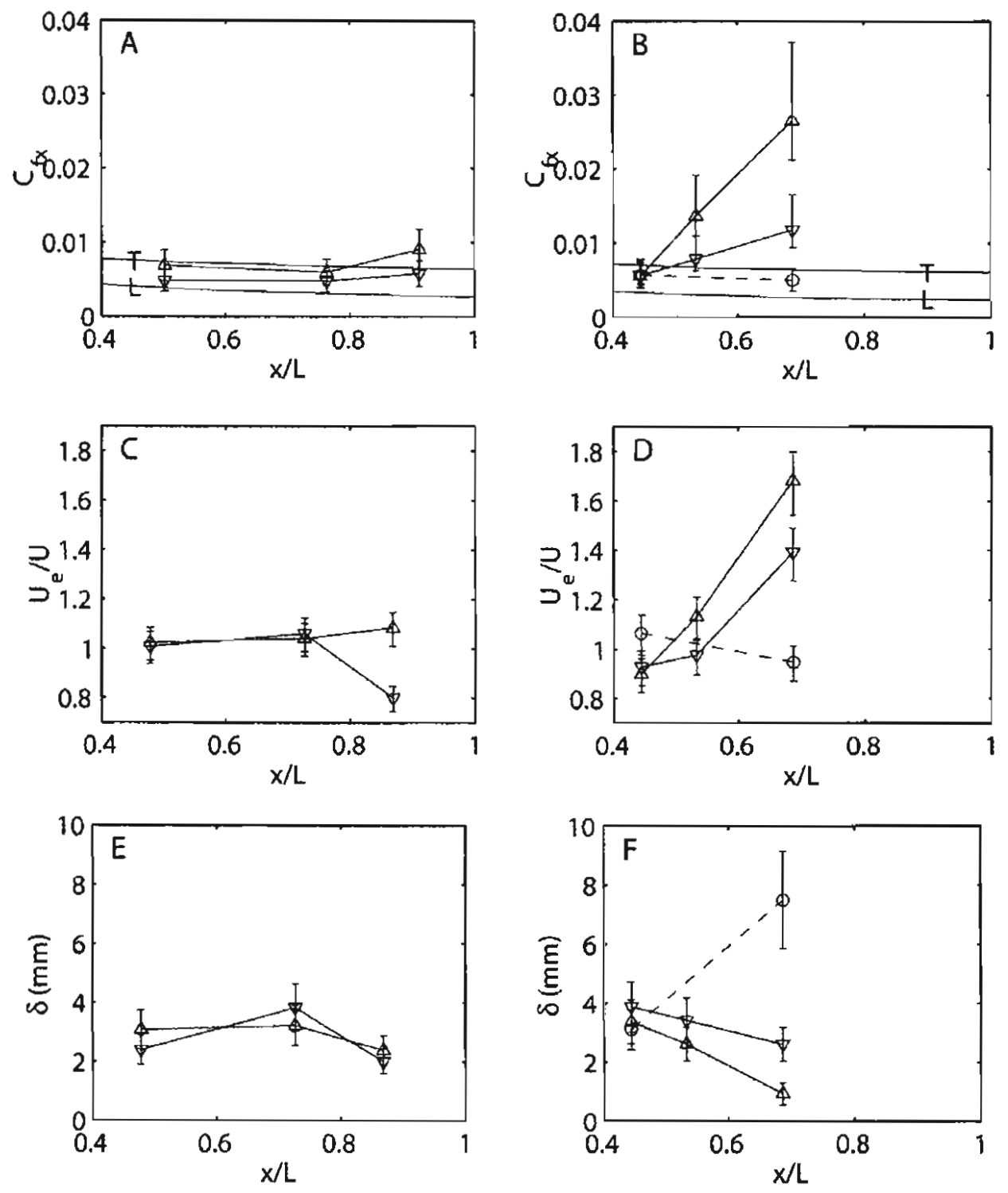

Fig. 3.6 Plots of time averaged local friction coefficients, $C_{f x}$, tangential velocity at the edge of the boundary layer, $U_{e}$, and boundary layer thickness, $\delta$, as a function of relative streamwise position, $x / L$, for the same data presented in Fig. 3.5. Time averages over periods during which the fish transverse body velocity was positive or negative are denoted by ' $\Delta$ ' and ' $\nabla$ ', respectively. Data from scup is presented in (A), (C) and (E). Dogfish data is presented in (B), (D), and (F). The rigid body case is denoted by ' 0 ' connected by dashed lines. Turbulent and laminar flat plate friction, labeled ' $T$ ' and ' $L$,' are included in (A) and (B) for comparison. On average, the data points for the opposite directions of transverse velocity, ' $\Delta$ ' and ' $\nabla$ ', represent half as many realizations as for the whole cycle averages of Fig. 3.5. Error bars are based on the maximum percent errors in the determination of the variables presented for the boundary layer profiles contributing to each data point. 
oscillates in phase with transverse body velocity (Fig. 3.6A,B). In the dogfish, the time average of $U_{e}$ increases with streamwise position on the body (Fig. 3.6D), suggesting a mean acceleration of both the boundary layer and the nearfield flow over the fish. In the scup, the time average of $U_{e}$ is close to $U$ for the entire region that was measured (Fig. 3.6C). In both species, $U_{e}$ oscillates in phase with transverse body velocity (Fig. 3.6C,D) and local friction (Fig. 3.6A,B) suggesting local oscillatory acceleration and deceleration in the nearfield and boundary layer. The boundary layer thickness over the posterior region of the dogfish, where local friction increases above flat plate friction, oscillates $180^{\circ}$ out of phase with transverse body velocity (Fig. 3.6F). Oscillatory effects in $C_{f x}, U_{e}$, and $\delta$ are more pronounced in the anguilliform swimmer than in the carangiform swimmer. Finally, the behavior of $C_{f x} U_{e}$, and $\delta$ in the rigid-body case is opposite to that in the swimming dogfish (Fig. 3.6B,D,F), while scup data show some similarity to the rigid case.

Uncertainties in $C_{f x}, U_{e}$, and $\delta$ were determined to be approximately $\pm 31 \%, \pm 6 \%$, and $\pm 21 \%$, respectively, with some variation among specific trials depending on the quality of the flow realizations. For example, the rigid-body case of the dogfish has lower than average uncertainty in $C_{f x}( \pm 19 \%)$ due to the large number of images of the same event acquired; i.e. many particle pairs were sampled. Uncertainties were often greater in one direction than another. For instance, the uncertainty in $C_{f x}$ for the swimming dogfish was $+42 \%$ and $-21 \%$. Where appropriate, error bars are used to display the unique uncertainties of data points.

Data from scup swimming in the flume at swimming speeds ranging from 30 to $60 \mathrm{~cm} \mathrm{~s}^{-1}$ at a water temperature of $23.3 \mathrm{C}$ shows that, in the neighborhood of $x=0.5 \mathrm{~L}$, $C_{f x}$ falls within the range of values expected for flat plates (Fig. 3.7). The effects of transverse body surface velocity at this position are consistently small compared to more caudal positions (Figs. 3.6A, 3.7). Therefore, in some positions on fish $R e_{x}$ appears to be 


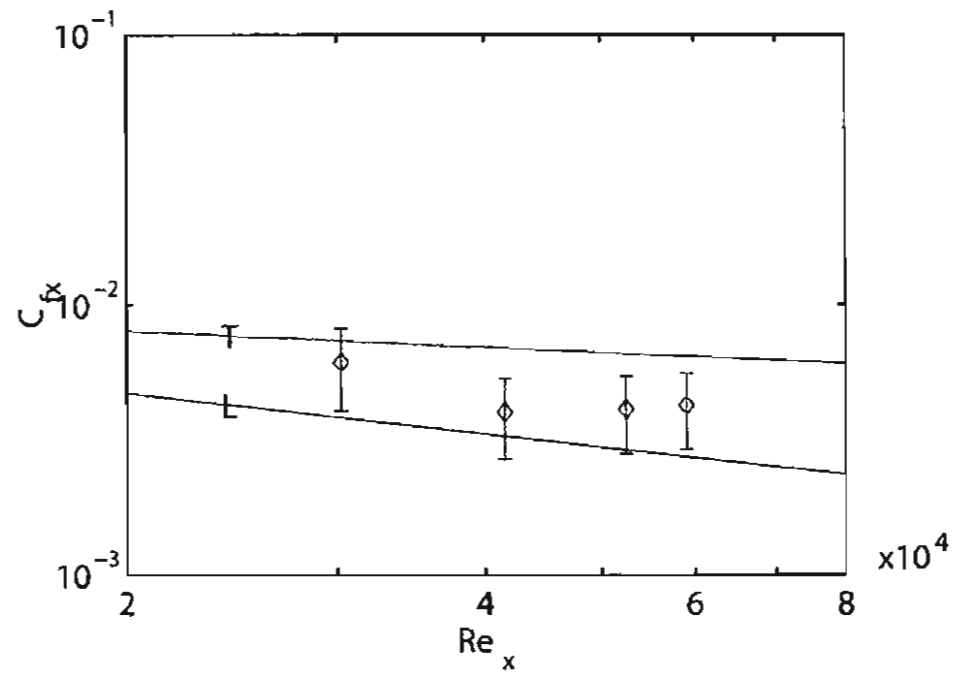

Fig. 3.7 Time averaged local friction coefficients, $C_{f x}$, vs. length Reynolds number, $R e_{x}$, at $x=0.50 L$ from several scup swimming sequences ranging in swimming speed from 30 to $60 \mathrm{~cm} \mathrm{~s}^{-1}$. No lines are drawn connecting these data points, ' $\diamond$,' since they do not represent the distribution of coefficients of friction along the body of a scup. The data at each $R e_{x}$ represent 9 - 10 boundary layer realizations. Error bars are based on the maximum percent errors in the determination of the slope of the linear sublayer, i.e. the wall shear stress, for the boundary layer profiles contributing to each data point. 
sufficient to predict local friction, whereas at other positions local friction deviates from flat plate friction and oscillates significantly.

\subsubsection{Oscillatory behavior of the boundary layer}

Oscillations in $C_{f x}, U_{e}$ and $\delta$, were highly correlated to the transverse velocity of the body surface (Fig. 3.6). Local friction and $U_{e}$ tend to be highest when the fish surface is moving into the fluid and lowest when the surface is retreating from the fluid; $\delta$ behaves in the opposite manner. A more highly resolved picture of the relationships between $C_{f x}, U_{e}, \delta$, and $V_{e}$ vs. body phase was obtained using polar phase plots for the dogfish swimming $20 \mathrm{~cm} \mathrm{~s}^{-1}$ (Figs. 3.8,3.9). $C_{f x}$ and $U_{e}$ are roughly in phase. Boundary layer thickness is roughly $180^{\circ}$ out of phase with $C_{f x}$. Normal flux oscillates in roughly $180^{\circ}$ out of phase with transverse body velocity. In addition to these previously described trends, the phase plots reveal a clockwise procession of maximum $C_{f x}, U_{e}, \delta$, and possibly $V_{e}$ with increasing relative position, $x / L$. This procession suggests that the distributions of these variables can be characterized as waves traveling along the body of the fish with wavelengths and speeds different from those of the body wave. The details of these 'distribution waves' will be discussed below.

\subsubsection{Oscillation of normal velocity}

Not only was $V_{e}$ observed to oscillate with body motion, but sequences of normal velocity profiles in both scup and dogfish swimming in the flume also revealed oscillation throughout the entire profile (Fig. 3.10). In both species, the sign of the normal velocity throughout the boundary layer is $180^{\circ}$ out of phase with transverse body surface velocity. As the body surface moves into the fluid, normal velocity is negative. During retreat, it is positive. At this short distance from the fish surface, incompressibility and continuity predict that this behavior is not simply a relative velocity effect. Furthermore, if the effect were strictly due to relative motion, the v-profiles 

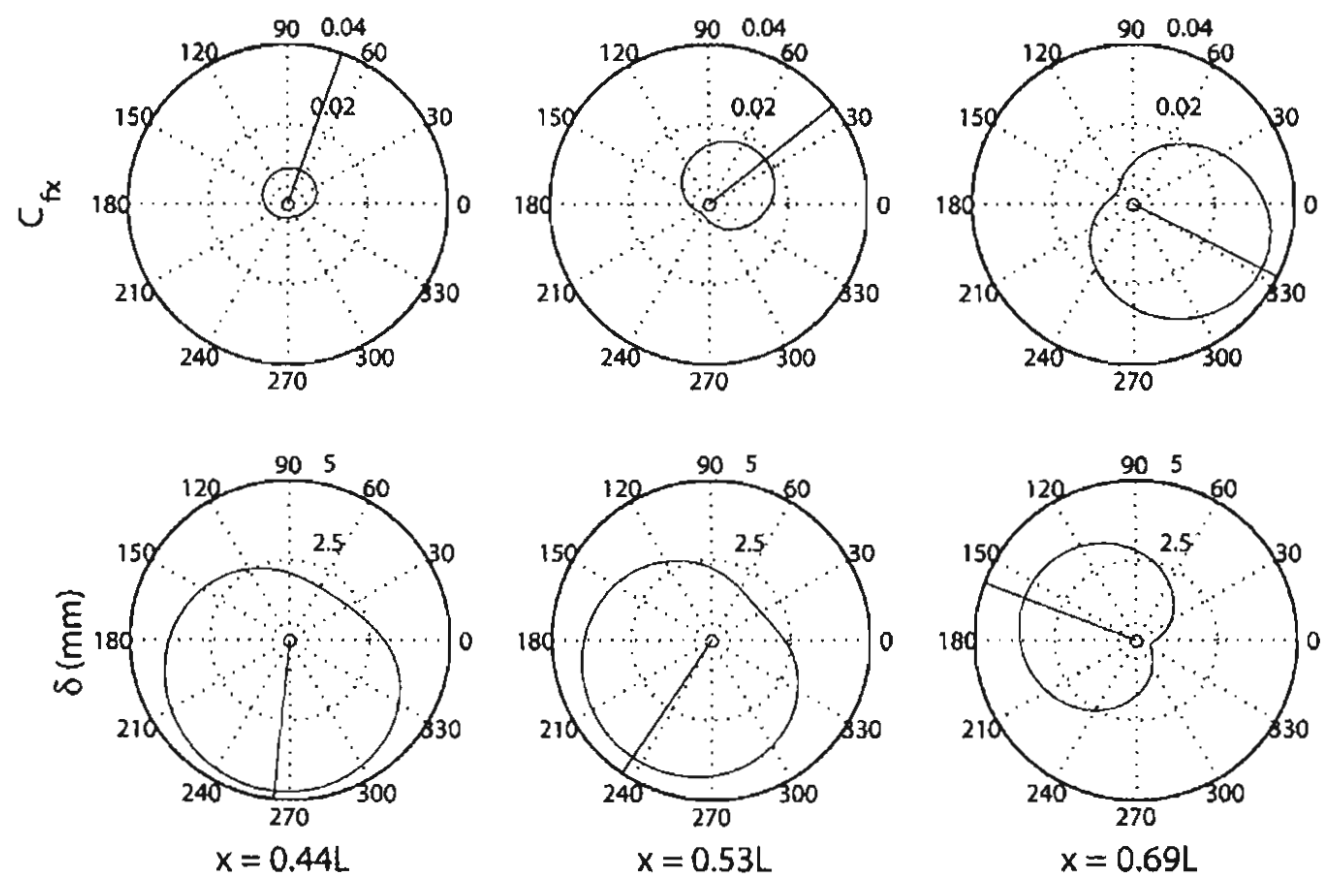

Fig. 3.8 Phase plots of local friction coefficients, $C_{f x}$, and boundary layer thickness, $\delta$, from 10 swimming sequences of the dogfish at the same swimming speed, $U=20 \mathrm{~cm}$ $\mathrm{s}^{-1}$, at three streamwise positions, representing 100 boundary layer realizations. The three positions along the body examined were, $x=0.44 \mathrm{~L}, 0.53 \mathrm{~L}$ and $0.69 \mathrm{~L}$. Each phase plot presents the behavior of a particular boundary layer variable vs. body phase measured at a particular position along the fish. Crest of the body surface corresponds to phase, $\phi=90^{\circ}$; trough corresponds to $\phi=270^{\circ}$. Time and phase increase in the counterclockwise direction, and radial distance expresses the magnitude of the boundary layer variable plotted. The radial scaling is printed between the angular positions $60^{\circ}$ and $90^{\circ}$. A solid radius is drawn on each phase plot to mark the phase of the maximum value of the variable displayed. Consider the plot of $C_{f x}$ at $x=0.69 \mathrm{~L}$. At $\phi=0^{\circ}$, the body is cycling from trough to crest, and $C_{f x}$ is equal to 0.033 . The highest positive transverse body velocities occur near this phase. As the phase reaches $90^{\circ}$, the body reverses direction. $C_{f x}$ decreases, reaching a minimum of 0.005 near $\phi=150^{\circ}$. At trough, $\phi=270^{\circ}$, friction is increasing and reaches a maximum near $\phi=330^{\circ}$ as the body is thrust toward the fluid. The cycle then repeats itself. The set of three plots for each variable are drawn to the same scale so that magnitudes as well as phase relationships can be compared. For example, one can observe the mean streamwise increase in $C_{f x}$ noting the progressive increase in area enclosed by the plotted curves. These curves are $4^{\text {th }}$ degree polynomial fits of the boundary layer data. They are constrained to be periodic, but not sinusoidal, by equalizing function values and slopes at the cycle beginning and end. This method of fitting the data allows for asymmetric phase plots. 

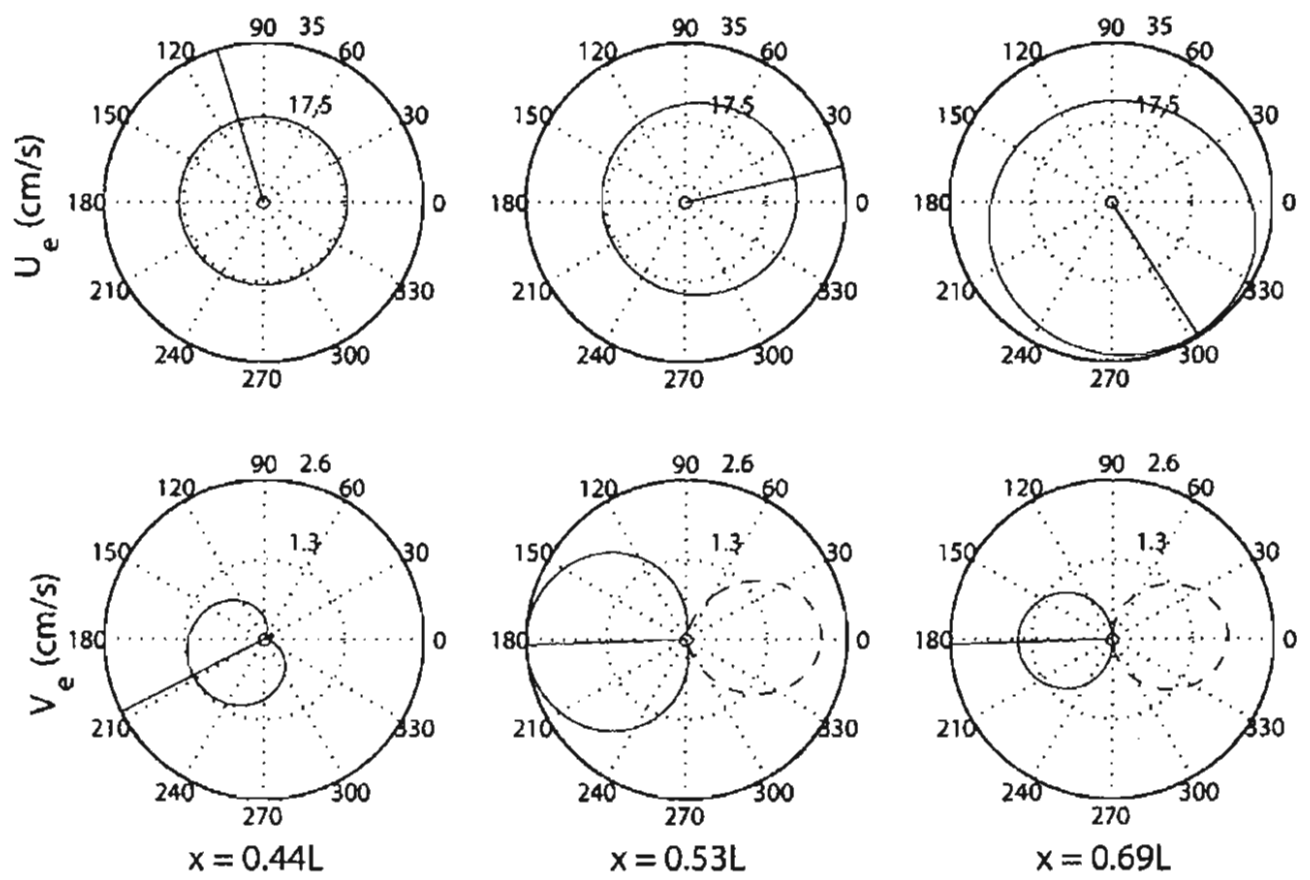

Fig. 3.9 Phase plots of tangential and normal velocity at the edge of the boundary layer, $U_{e}$ and $V_{e}$, for the same 10 swimming sequences of dogfish swimming (20 $\left.\mathrm{cm} \mathrm{s}^{-1}\right)$ as in Fig. 3.8. The details of the construction of the phase plots are described in the legend of Fig. 3.8. For $V_{e}$, the solid lines represent positive values, or outflow, and the dashed lines, negative, or inflow. The increasing area enclosed by the plots of $U_{e}$ show mean streamwise acceleration as shown in Fig. 3.6D. 

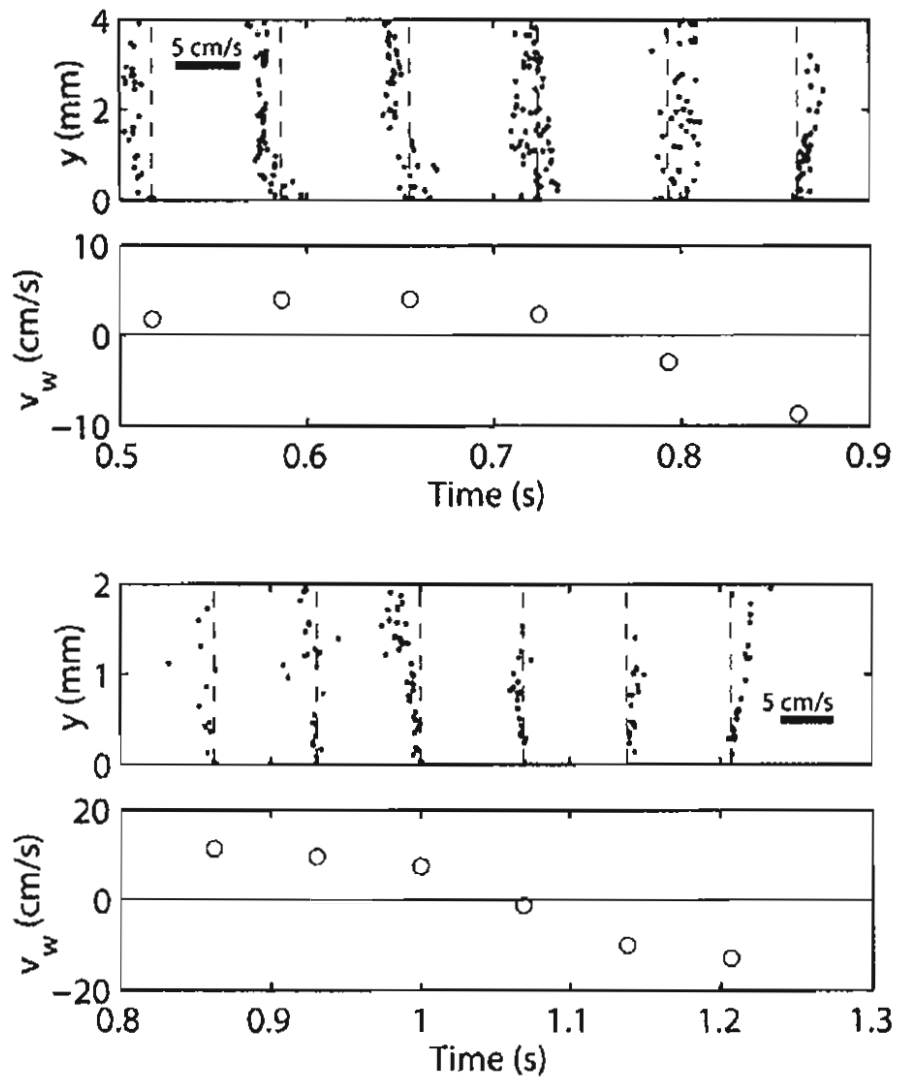

Fig, 3.10 Time series of normal velocity profiles, v-profiles, from scup ( $x=0.77 L$, $\left.U=30 \mathrm{~cm} \mathrm{~s}^{-1}\right)$ and dogfish $\left(x=0.53 L, U=20 \mathrm{~cm} \mathrm{~s}^{-1}\right)$ swimming sequences in the flume, together with transverse body surface velocity, or wall velocity, $v_{w}$. The dashed vertical lines represent the $\mathrm{v}=0$ axis for each profile and are positioned at the times of the realizations. These times correspond to the times at which $v_{w}$ was determined. Velocities within the profiles can be determined on the basis of the velocity scale bar shown and the respective $v=0$ axis-positive to the right, negative to the left (e.g. Fig. 3.4D). 
would be expected to exhibit velocities equal to the transverse wall velocity throughout the boundary layer.

\subsubsection{Incipient separation}

While no boundary layer separation was observed in the fish studied, incipient separation was seen in 6 swimming sequences. Figs. 3.11 and 3.12 show examples of incipient separation in scup in both still and flowing water. The example from still water (Fig. 3.11) dramatically demonstrates the highly inflected, low shear boundary layer profile shape of incipient separation. Our data show that incipient separation occurs after wall velocity, $v_{w}$, becomes negative, and that friction essentially drops to zero where the inflected profiles occur.

In the flume, a time sequence of the boundary layer behavior was obtained that included incipient separation (Fig. 3.12). As in the still water example (Fig. 3.11), incipient separation occurs close to where wall velocity goes negative. Local friction decreases noticeably. The time sequence suggests that the inflected boundary layers, which occur at troughs, are stabilized as the body phase cycles toward the subsequent crests. In the flume, instances of inflected boundary layers were observed twice in separate sequences of scup swimming $30 \mathrm{~cm} \mathrm{~s}^{-1}$ and once in the dogfish swimming $20 \mathrm{~cm}$ $s^{-1}$.

\subsubsection{Total skin friction and friction coefficients}

Table 3.1 presents calculations of total body friction drag and corresponding friction coefficients for scup (swimming) and dogfish (swimming and rigid). Power required to overcome friction drag is presented. In Fig. 3.13, the coefficients of friction are plotted vs. Re together with flat plate friction for comparison. The coefficients of friction for swimming scup and the rigid dogfish fall within the range of flat plate friction 

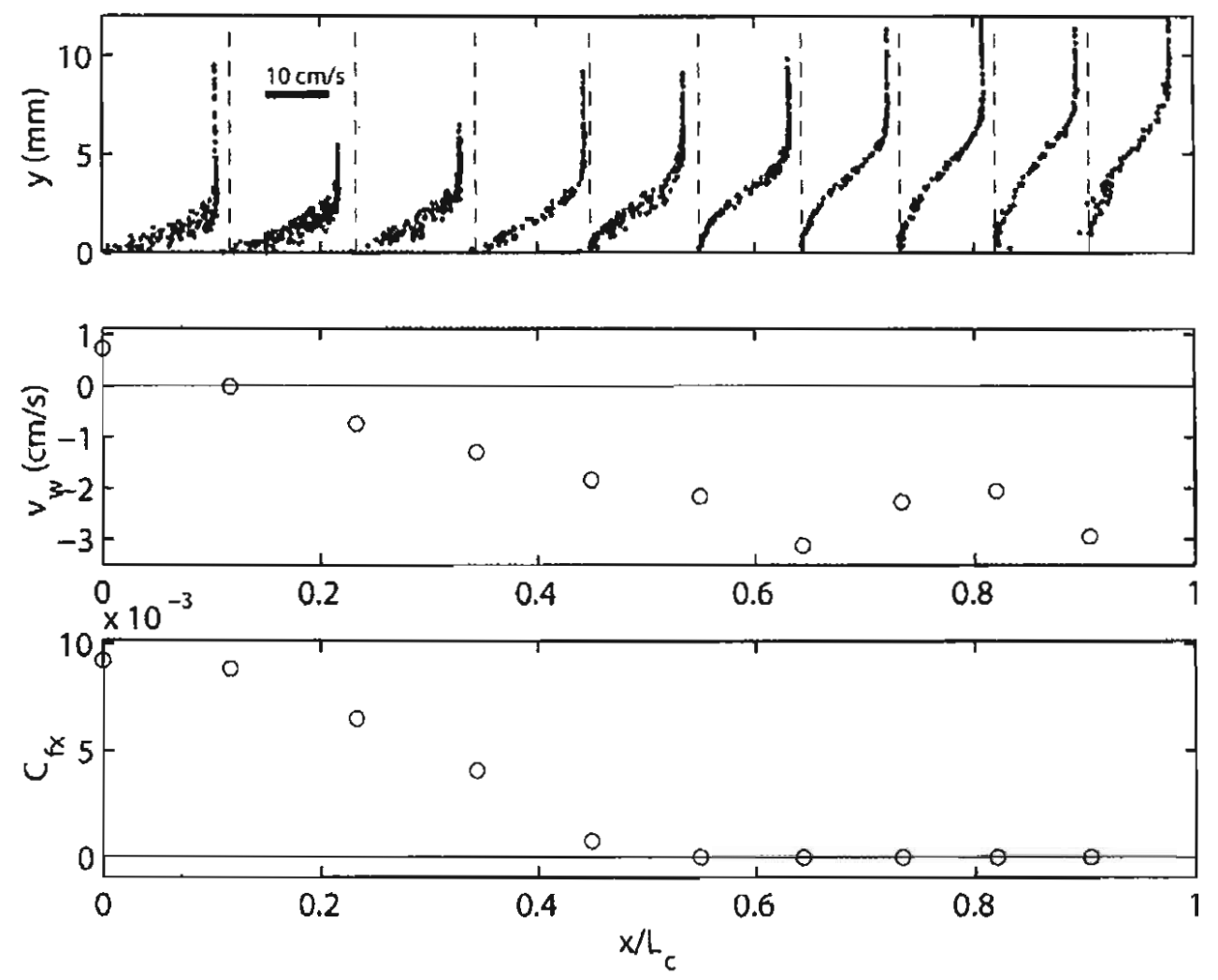

Fig. 3.11 Boundary layer development (i.e. $u$-profiles), transverse body surface velocity, $\mathrm{v}_{w}$, and local friction coefficients, $C_{f x}$, over a swimming scup showing incipient separation. The $u$-profiles shown were observed $5 \mathrm{~cm}$ above the centerline of the fish and spanned from the leading edge to the trailing edge of the body for a chord length, $L_{c}$, of $9 \mathrm{~cm}$. The dashed vertical lines represent the $u=0$ axis for each profile and are positioned at the relative streamwise position on the fish, $x / L_{c}$, of the given realization. These positions correspond to the positions at which $\mathrm{v}_{w}$ and $C_{f x}$ were determined. Velocities within the profiles can be determined on the basis of the velocity scale bar shown and the respective $u=0$ axis-positive to the right, negative to the left (e.g. Fig. 3.3A). The decreased distance between successive $u=0$ axes reveals that the fish was decelerating. The very quiet, or uniform, flow just outside of the boundary layer shows that this is a still water trial. 

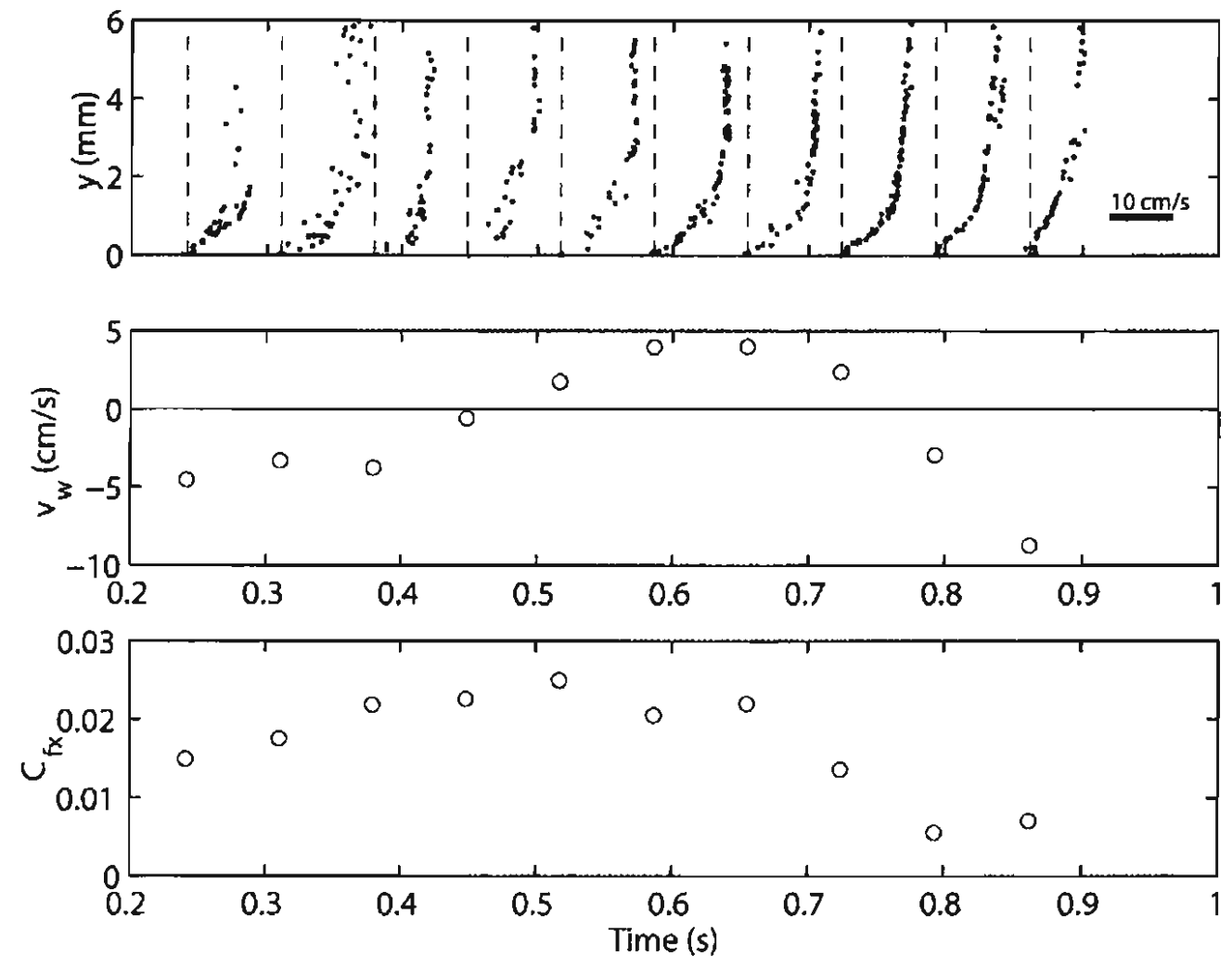

Fig. 3.12 Time series of tangential velocity profiles, $u$-profiles, showing incipient separation at $x=0.77 \mathrm{~L}$, near the peduncle of a scup swimming $10 \mathrm{~cm} \mathrm{~s}^{-1}$ in the flume. Approximately one locomotory cycle is shown as revealed by the plot of transverse wall velocity, $\mathrm{v}_{w}$. Incipient separation occurs most clearly in the two profiles measured between $t=0.7$ and $0.8 \mathrm{~s}$. The data at the start of the time series, although they are of poor quality, are attached and stable. The dashed vertical lines represent the $u=0$ axis for each profile and are positioned at the times of the realizations. Velocities within the profiles can be determined on the basis of the velocity scale bar shown and the respective $u=0$ axis-positive to the right, negative to the left (e.g. Fig. 3.3A). 
Table 3.1 Total drag calculations based on measured wall shear stress distributions over scup and dogfish

\begin{tabular}{|c|c|c|c|c|c|}
\hline & & $\begin{array}{l}\text { M. Canis } \\
\text { rigid-body }\end{array}$ & $\begin{array}{l}\text { M. canis } \\
\text { flume }\end{array}$ & $\begin{array}{l}\text { S. chrysops } \\
\text { still water }\end{array}$ & $\begin{array}{l}\text { S. chrysops } \\
\text { flume }\end{array}$ \\
\hline Swimming speed, $U$ & $\mathrm{~cm} / \mathrm{s}$ & 20 & 20 & 10 & 30 \\
\hline Temperature, $T$ & $\mathrm{C}$ & 22.8 & 22.8 & 23.3 & 23.3 \\
\hline Lateral body area, $A$ & $m^{2}$ & 0.0213 & 0.0213 & 0.0206 & 0.0206 \\
\hline Length, $L$ & $\mathrm{~cm}$ & 44.4 & 44.4 & 19.5 & 19.5 \\
\hline Mass, $M$ & $\mathrm{~kg}$ & 0.218 & 0.218 & 0.166 & 0.166 \\
\hline Measured friction drag, $D_{f}$ & N & 0.0033 & 0.0064 & 0.0013 & 0.0067 \\
\hline Theoretical flat plate friction drag, $D_{H}$ & N & 0.0018 & 0.0018 & 0.0007 & 0.0044 \\
\hline Measured friction drag coefficient, $C_{f}$ & & 0.0076 & 0.0146 & 0.0127 & 0.0071 \\
\hline Theoretical friction drag coefficient, $C_{n}$ & & 0.0041 & 0.0041 & 0.0068 & 0.0047 \\
\hline$D_{f} / D_{t}$ & & 1.8 & 3.6 & 1.9 & 1.5 \\
\hline$D_{f} /$ measured rigid body friction drag & & 1.0 & 1.9 & & \\
\hline Power required to overcome $D_{f}$ & $\mathrm{~mW}$ & na & 1.3 & 0.13 & 2.0 \\
\hline Mass of red muscle per mass of fish & & & $0.0332^{\star}$ & & $0.0209^{* *}$ \\
\hline Mass of red muscle & $\mathrm{kg}$ & & 0.0072 & & 0.0035 \\
\hline Power required per mass red muscle & W/kg & & 0.2 & & 0.6 \\
\hline Power available per mass red muscle & W/kg & & $>(3 \text { to } 8)^{\star \star \star}$ & & $>(3 \text { to } 8)^{* \star * *}$ \\
\hline
\end{tabular}

*Based on estimates from the data of Greer-Walker and Pull (1975) for spiny dogfish (Squalus acanthias), and the distributions of red muscle in scup (S. chrysops) from Zhang et al. (1996) and Pacific mackerel (Scomber japonicus) from Graham et al. (1983), see text

**Zhang et al., 1996

***Based on power outputs at in vivo conditions for scup ( $S$. chryspos) from Rome et al. (2000), and Swank and Rome (2001), see text 


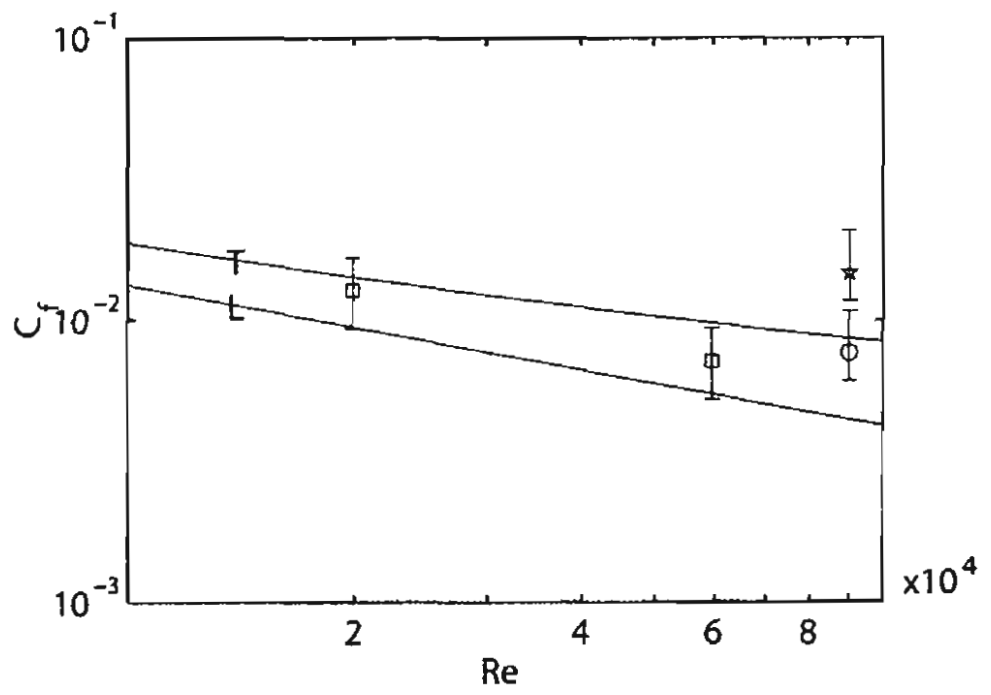

Fig. 3.13 Total coefficients of friction, $C_{f}$, vs. Reynolds number, $R e$, calculated for scup, ' $\square$ ', and dogfish, ' $\forall$ ' (Table 3.1), including the rigid body case of the dogfish, 'o.' Turbulent and laminar flat plate total friction coefficients, labeled ' $T$ ' and ' $L$,' are included. 
for laminar and turbulent flow. The coefficient of friction for the swimming dogfish falls above this range.

\subsection{Discussion}

\subsubsection{The nature of the fish boundary layer}

In the most general sense, the boundary layer of swimming fish can be characterized by streamwise trends and local oscillations in $C_{f x}, U_{e}, \delta, V_{e}$ and overall profile shape. Streamwise trends proved to be highly dependent on swimming mode (Fig. 3.6). Local oscillations of boundary layer related variables occurred similarly in both the dogfish and scup, though the amplitudes of oscillation were greater in the dogfish. The data reveal that all of these behaviors can be understood from the perspective of two superimposed fluid accelerations: mean streamwise acceleration and local oscillatory acceleration that is correlated to the transverse motion.

The streamwise increase of $U_{e}$ in the dogfish is evidence of mean streamwise acceleration of the nearfield and boundary layer flow. The time averaged values of $C_{f x}$ increase and $\delta$ decrease as would be expected in a boundary layer under an accelerating exterior flow. No significant mean streamwise acceleration was observed in scup; however, the nearfield flow was not observed to decelerate either. The absence of mean acceleration over the scup follows from the tendency of carangiform swimmers to produce the majority of their thrust at the caudal fin. Mean streamwise acceleration is a sign of thrust production. The difference between scup and dogfish in this regard can be understood considering the relatively small wave amplitudes present in carangiform swimmers. Studies of swimming performance after complete caudal fin amputation (Breder, 1926; Gray, 1968; Webb, 1973) show that carangiform swimmers are able to compensate surprisingly well for the loss of fin thrust by increasing body wave amplitude 
and frequency. The observed differences in amplitude and frequency after complete amputation suggest a change in swimming mode on the part of the fish. Mean streamwise acceleration of the nearfield might be expected to occur over a larger portion of the body in these fish since they have only their bodies to produce thrust in the amputated state. However, it does not follow that carangiform swimmers actually do use their body wave to produce a significant amount of thrust forward of the caudal fin. When the caudal fin is amputated, one would not expect the fish to use the same body motion to swim as it did with the caudal fin intact. Therefore, it would be tenuous to conclude that since a carangiform swimmer with its caudal fin amputated uses body based thrust to swim that the same is true when the tail has not been removed. Our data suggest low body based thrust in scup compared to caudal fin based thrust since mean streamwise acceleration of the nearfield fluid forward of the peduncle, which would be the evidence of the body producing thrust with the body forward of the peduncle, was not observed.

In both scup and dogfish, $U_{e}$ and $\delta$ were observed to oscillate $180^{\circ}$ out of phase with each other. $C_{f x}$ behaves as would be expected according to the first order approximation, $\tau_{o} \cong \mu U_{d} \delta$ (Eq. 2.14). This and the concurrent oscillation of the v-profile (Fig. 3.10) reveal a cycle of local tangential acceleration and deceleration of the boundary layer at any given position along the fish. As explained earlier, positive and negative normal velocity relative to the body at the edge of the boundary layer are evidence of normal flux out of and into the boundary layer, respectively. In general, tangential flow accelerates as the body cycles from trough to crest, and decelerates as the body cycles from crest to trough.

One might argue that normal flux exhibited by the v-profile is simply the observation of relative motion due to the surface fixed coordinate system, but that would be true only if one were focusing on the far-field, where there is negligible impact on the flow due to the fish. Allen (1961) apparently uses this far-field concept to explain his 
supposed observation of boundary layer thickness oscillation. In contrast, the normal flux revealed in Fig. 3.10 occurs at the level of the nearfield and boundary layer. Therefore, it is not merely relative fluid motion. If this were so, we would expect the normal velocity to match the movement of the fish surface with decreased distance from the fish, due to the incompressibility of water and the no-flux boundary condition at the fish surface. The fact that the opposite effect is observed at the edge of the boundary layer indicates tangential and/or cross-stream boundary layer acceleration.

\subsubsection{Wave-like distributions of boundary layer variables and pressure}

As mentioned earlier, the oscillatory behavior of $C_{f x}, U_{e}, \delta$, and $V_{e}$ with relative position along the fish suggest that the streamwise distributions of these variables can be represented as traveling waves moving in the same direction as the fish body wave. The clockwise procession of maximum values in the phase plots reveal an ever increasing downstream shift in the streamwise distributions of the variables with respect to the phase of the body traveling wave (Figs. 3.83 .9 ). Regular periodic behavior of these variables at fixed positions on the fish reveals that these 'distribution waves' and the body traveling wave have the same frequency, f. Since $c=\lambda f$, the increasing streamwise phase shift of the variable distributions with respect to the body wave is therefore due to the distribution waves having a longer wavelength, $\lambda$, and higher wave speed, $c$, than the body traveling wave.

Wave speeds and wavelengths of the distribution waves can be determined from the streamwise rate of procession. The procession of local friction is approximately $32^{\circ}$ as $x$ changes from $0.44 L$ to $0.53 L$. Between $x=0.53 L$ and $0.69 L$, procession is approximately $65^{\circ}$. Therefore, the ratio of procession to change in body position, i.e. the rate of procession, is roughly constant. Taking the procession of all the variables in Figs. 3.8 and 3.9 , procession along the body wave per body length is about $400^{\circ}$, i.e. 7.0 rad. If 
procession per unit length is roughly constant, then the wavelength and wave speed of the streamwise distribution of local friction are roughly constant.

The item of interest is how far the friction distribution travels relative to the body wave. Let $\phi_{0}$ be the procession of the friction distribution through the body wave in radians per unit body length traveled by the friction distribution. If the friction distribution moves a distance $\Delta x_{F}$ along the body, then it moves through the body wave a distance, $\Delta x_{p}$, of

$$
\Delta x_{P}=\phi_{o} \Delta x_{F} \frac{\lambda_{B}}{2 \pi}
$$

where $\lambda_{B}$ is the body wavelength. $\Delta x_{P}$ can be thought of as the 'length of procession'. The distance moved by the friction distribution $\Delta x_{F}$ is equal to $\Delta x_{P}$ plus the distance the body wave traveled, $\Delta x_{B}$, that is,

$$
\Delta x_{F}=\Delta x_{B}+\phi_{o} \Delta x_{F} \frac{\lambda_{B}}{2 \pi}
$$

Therefore, the distance moved by the friction distribution relative to the distance traveled by the body wave is

$$
\frac{\Delta x_{F}}{\Delta x_{B}}=\frac{1}{1-\phi_{o} \frac{\lambda_{B}}{2 \pi}}
$$


This is also the ratio of the speed and wavelength of the friction distribution along the body relative to the body wave speed and wavelength. In the dogfish, swimming $20 \mathrm{~cm}$ $\mathrm{s}^{-1}$, the measured body wavelength, $\lambda_{B}$, was $27 \mathrm{~cm}$, and body length, $L$, was $44.4 \mathrm{~cm}$. Substituting this and $\phi_{o}=7.0 \mathrm{rad} / 44.4 \mathrm{~cm}$ into Eq. 3.5 leads to a ratio of 3.1 , that is, the friction distribution travels 3.1 times faster along the body than the body wave. Boundary layer thickness exhibits the same rate of procession. $U_{e}$ appears to have the same rate of procession despite the larger phase shift between $x=0.44 \mathrm{~L}$ and $0.53 \mathrm{~L}$. There is so little variation in $U_{e}$ at $x=0.44 L$ that it is possible that there is significant error in the determined phase of the maximum. Finally, normal flux exhibits the same rate of procession for $x=0.44 L$ to $0.53 L$, but very little procession occurs between $x=$ $0.53 L$ and $0.69 L$.

Taken together, the general procession of all four variables (Figs. 3.8,3.9) is evidence of a traveling pressure distribution over the fish. Boundary layer thinning, negative normal flux and the increase in $U_{e}$ can be understood as being linked to accelerations of the boundary layer and nearfield flow. These accelerations, in turn, can be thought of as being driven, at least in part, by pressure gradients. We assume here that maxima in boundary layer acceleration, as evidenced by $C_{f x}, U_{e}, \delta$, and $V_{e}$, are indicative of maxima in pressure gradient. Therefore, the pressure distribution around the fish behaves like the distributions of these variables in wavelength and wave speed.

From this assumption, the data in Figs. 3.8 and 3.9 predicts that when the body wave crest has reached the peduncle of the fish and the pressure maximum resides on the rearward facing surface of the caudal fin. In the same way, pressure minima shift to positions on the forward facing surfaces of the posterior body and caudal fin. This orientation on pressure maxima and minima would result in thrust production over the posterior half of the body. On the anterior half of the body, pressure maxima occur on the forward facing surfaces and pressure minima occur on the rearward facing surfaces, as is normally the case for a non-thrust producing body moving through a fluid. 
Therefore, the pressure distribution suggested by the behavior of the boundary layer, independent of the assumption of thrust production, is in elegant agreement with the expected hydrodynamics in fish. This suggests that analysis of the boundary layer may be an invaluable tool in the investigation of the hydrodynamics of undulatory swimming.

\subsubsection{Drag enhancement and drag reduction}

Friction drag on the swimming dogfish is higher than rigid-body friction drag, as predicted by Lighthill (1971). The data in Table 3.1 reveal that the friction drag on a swimming dogfish is 3.6 times the theoretical flat plate friction drag, and 1.9 times the measured rigid-body friction drag. The difference in these two ratios is due to the fact that the friction drag on the rigid dogfish is greater than flat plate friction. In scup, drag enhancement was observed to be less pronounced than that observed in the dogfish, and measured friction drag was calculated to be only $1.5-1.9$ times theoretical flat plate friction, thus it is not certain that friction drag on the swimming scup is higher than that on a rigid scup. Friction on a rigid scup was not measured. Interestingly, the behaviors of $C_{f x}, U_{e}$ and $\delta$ for the swimming scup are not dramatically different from the rigid dogfish (Figs. 3.5,3.6). This may be due to the fact that a carangiform swimmer deviates less from a rigid body than does an anguilliform swimmer (Breder, 1926).

Fig. 3.6 reveals that enhanced friction drag can be linked to boundary layer thinning in both species, thus supporting the hypothesis of Bone and Lighthill. However, greater values of $U_{e}$ in the swimming dogfish compared to the rigid-body case suggests that mean streamwise acceleration of the nearfield is a second, independent mechanism of enhanced friction drag. While the hypothesis of Bone and Lighthill requires transverse motion to operate, any streamwise acceleration of the flow around a body can result in increased friction drag, regardless of transverse motion. Of course, there would be no acceleration of the flow around a fish if the fish were not waving its body, but the BoneLighthill hypothesis is more closely linked to the transverse body motion than this second 
hypothesis. The streamwise acceleration hypothesis is linked to the mean flow field arising from a propulsive system that generates thrust over a significant portion of its body. This would occur whether or not the body used undulatory propulsion as long as the thrust producing elements were close to the body surface. A non-undulatory example in animal swimming might be the squid, for example $L$. pealei. This organism propels itself using a high velocity jet that exits beneath its arms. The accelerated flow over the surface of the arms undoubtedly leads to enhanced friction drag.

It might be argued from the dogfish data at $x=0.69 L$ that only the Bone-Lighthill hypothesis is acting. At this position, boundary layer thickness over the swimming dogfish is approximately $1 / 4$ times that over the rigid dogfish (Fig. 3.6F), while the local friction on the swimming dogfish is 4 times that on the rigid dogfish (Fig. 3.6B). The linear approximation, $\tau_{o} \cong \mu U_{\Theta} / \delta$, suggests that boundary layer thinning alone is enough to explain the enhanced friction drag. This arguments fails, however, because the boundary layer at $x=0.69 \mathrm{~L}$ on the rigid dogfish was clearly turbulent, while the boundary layer on the swimming dogfish at this position appeared to be laminar for the majority of the time. The shapes of laminar and turbulent profiles are radically different and the approximation, $\tau_{o} \cong \mu U_{e} / \delta$, breaks down. The Bone-Lighthill hypothesis does not include the effects of such differences in boundary layer condition. Their estimate that boundary layer thinning can lead to swimming friction drag that is $3-5$ times greater than rigid-body friction drag is made assuming that the boundary layer flow condition is the same in both the swimming and rigid-body cases. If the boundary layer on the rigid dogfish were laminar, rather than turbulent, it would have been up to $40 \%$ thinner and had a lower local friction. Therefore, the calculated drag enhancement at $x=0.69 \mathrm{~L}$ would be greater than 4 times, while the boundary layer thinning would be less than 4 times. Then, by the linear approximation of $\tau_{o}$, the degree of boundary layer thinning between the rigid and swimming cases would not be enough to account for the increase in local friction. Furthermore, the fact that $U_{e}$ at $x=0.69 \mathrm{~L}$ for the swimming dogfish is 1.6 
times that for the rigid dogfish (Fig. 3.6D), makes it very difficult to argue that mean streamwise acceleration has no impact on the local friction at this position.

In scup, no obvious mean streamwise acceleration of the nearfield flow was observed (Fig. 3.6C), while friction increases by almost a factor of two between $x=$ $0.77 \mathrm{~L}$ and $x=0.91 \mathrm{~L}$. Fig. 3.6E reveals that boundary layer thickness decreases by almost $50 \%$ between these two positions, and the Lighthill-Bone hypothesis can account for the streamwise increase in local friction. The lack of mean streamwise acceleration in scup may therefore explain the lower drag enhancement in scup (Fig. 3.6A), illustrating a way in which the carangiform mode of swimming leads to increased efficiency. Lighthill (1969) details other beneficial aspects of the carangiform mode. In contrast, anguilliform swimmers use large amplitude motions over a significant portion of the body to accelerate flow (Figs. 3.6D , 3.9) and produce thrust anterior to the caudal fin. The price is significantly increased drag (Figs. 3.6B, 3.8) and, most likely, decreased efficiency.

\subsubsection{Drag reduction mechanisms}

The suggestion of enhanced drag, especially in dogfish, does not exclude the possibility that drag reducing mechanisms are operating in fish swimming. Two possible mechanisms observed by Taneda and Tomonari (1974) were suggested in fish boundary layers. They are form drag reduction by delayed separation and friction drag reduction by partial or total laminarization. Fish boundary layers strongly suggested the former effect, which will be discussed in detail below. As to laminarization, both laminar and turbulent boundary layer flow were observed under various circumstances. Not surprisingly, turbulent boundary layers occurred at lower than critical Reynolds numbers in the flume, but flume turbulence did not cause turbulent boundary layer flow over the whole fish at all times as has been suggested by Webb (1975). Even at high Reynolds numbers, the boundary layer appeared, in some cases, to oscillate between laminar (in troughs) and turbulent flow (on crests) as in the waving plate of Taneda and Tomonari 
(1974). These observations reveal that although fish do not completely suppress turbulence, there is likely some stabilization enacted by the body motion, which could lead to energy savings by some friction drag relief.

The observation of turbulent boundary layer flow in certain circumstances presents the possibility yet another drag reducing mechanism--turbulent boundary layer drag reduction by surface features, such as mucus or riblets. Dermal ridges on sharks have been shown to act as riblets in reduction of turbulent boundary layer drag (Reif, 1982; Bechert et al., 1985). There is also evidence that the mucus of fish can reduce turbulent boundary layer drag in the same way that large polymer additives have been observed to do (Webb and Weihs, 1983). These mechanisms only operate when the boundary layer is turbulent. Fish would not be expected purposely to trigger turbulent boundary layer flow to gain drag reduction by such methods-a laminar boundary layer would be preferable. Nevertheless, fish may benefit somewhat from such mechanisms, since their boundary layers do show instances of being turbulent.

The apparent conflict of suggesting that both drag reduction and enhanced friction drag occur simultaneously in undulatory swimming arises from a subtlety in the definition of drag reduction in undulatory swimming. Drag reduction should not simply be thought of as an improvement in the swimming state over the rigid body. By that definition, there is certainly no friction drag reduction (Figs. 3.5, 3.6B). More accurately, drag reduction is an improvement within the realm of the swimming state. For example, consider the proposed turbulent drag reduction by dermal ridges, or riblets, in sharks. If riblets lead to a reduction in drag in sharks, we would expect lower drag on a live fish compared to that of an identical robotic fish without riblets swimming with identical kinematics. It would not make sense to compare the drag on a rigid body, with or without riblets, to the drag on a swimming fish with riblets. In light of the friction drag enhancement confirmed by our observations, it is likely that the swimming fish, even with riblets, would have a higher drag. The decision of whether or not drag reduction is 
present really has nothing to do with the stretched-straight case unless the purpose is to test the advantages of fish-like vehicles or to study coasting in fish, as in the investigation of burst and coast swimming by Weihs (1974). For this same reason, even the term 'drag enhancement' needs to be used carefully. In general, fast swimming fish and cetaceans need to undulate some portion of their bodies in order to swim, and the rigid-body state is not an option. Hydrodynamic optimization in biology must be viewed within this constraint.

No separation of flow was observed in scup or dogfish. Separation of flow is the result of momentum losses, or decelerations, that eventually prevent the continued streamwise progress of the boundary layer fluid along the body surface. These losses in momentum are generally due to pressure gradients working against the streamwise fluid motion. Such pressure gradients are referred to as adverse. Boundary layer profiles from the caudal fin of a swimming scup revealed attached flow. Similarly, Taneda and Tomonari (1974) observed the flow on a waving plate to remain attached to the trailing edge. They hypothesized that acceleration of flow, which they observed along the waving plate, explained the prevention of separation observed. Such acceleration is evidence of a favorable, mean streamwise pressure gradient, opposite to that which would result in flow separation. The mean streamwise acceleration we have observed in dogfish suggests the same stabilization process. The similarity of the dogfish to the waving plate of Taneda and Tomonari (1974) is reasonable since the plate was operated at a swimming mode similar to the anguilliform mode.

In scup, although no obvious mean streamwise acceleration was observed, the fact that no significant mean deceleration of the nearfield and boundary layer flow occurred may explain why no separation was observed. Momentum is certainly being removed at the fish surface by friction, and since the flow over the fish does not decelerate, the addition of some potentially stabilizing momentum is sustaining the relatively constant streamwise flow over the fish. It is also possible that an oscillatory effect similar to the 
enhanced friction hypothesis of Lighthill and Bone is operating. If the diffusion of momentum out of the boundary layer as the fish surface cycles from crest to trough is slower than the production of momentum as the surface is thrust into the fluid, then there will be a net increase in boundary layer momentum. Boundary layer profiles signaling incipient separation were always observed during the crest to trough motion and were apparently stabilized as the surface moved from trough to crest.

The occasional appearance of incipient separation and subsequent stabilization may be evidence of complex flow manipulation on the part of the fish, which may be used to optimize the ratio of thrust to drag. Avoiding separation, a fish essentially eliminates form drag and increases the effectiveness of the caudal fin in thrust production. At the same time, more 'strongly attached' boundary layers mean higher wall shear stress and therefore increased friction drag. Perhaps fish tune swimming movements to take advantage of the lowered shear stress of a nearly separating boundary layer, while simultaneously benefiting from the reduced form drag and increased lift of fully attached flow. The inflected boundary layer profiles observed may be an example of the fish 'pushing the envelope' and, as the time sequence implies, the fish quickly corrects back toward the attached state. Fig. 3.5 reveals that drag enhancement in scup is significantly less than in the dogfish. The lower drag may be the result of the proposed optimization, since inflected boundary layers were more often observed in the carangiform swimming scup. However, the data from the dogfish may not have been sufficiently near the tail to test for the phenomenon.

Another explanation for the appearance of inflected profiles is some disturbance in the flow, but in this case one might have expected to see inflected boundary layers on both fish at several different positions. Instead, inflected profiles, with the exception of the single case in the dogfish, occurred near trailing edges. Regardless of the origin of the inflected profiles, it follows from the suggested correction mechanism that fish are able to sense near-wall hydrodynamic parameters, such as shear and pressure, and 
quickly adjust muscular control of swimming motions to optimize efficiency. For many years, it has been suggested that the neuromasts of the fish lateral line system are capable of just such flow sensing (Coombs and Montgomery, 1999).

The boundary layer of swimming fish suggests a favorable trade-off between thrust production, separation control and friction drag in undulatory swimming. The similarity of our data to those of Taneda and Tomonari (1974) on a waving plate lends weight to numerical and experimental studies focusing on this simplified geometry. Perhaps small variations in swimming parameters would require higher shear profiles to insure attachment, or lead to changes in the duration of laminar periods in the boundary layer oscillation thereby increasing or decreasing friction drag. Simultaneous effects on form drag and thrust production would doubtlessly occur in this highly non-linear system. It should be noted that 'optimum' is not necessarily synonymous with efficient, since issues, such as escape may be equally important. Knowledge of the boundary layer brings us closer to answering an important question regarding optimization in undulatory locomotion: What slight perturbations of fish swimming motions lead to a more or less advantageous locomotory mechanism?

\subsubsection{Two-dimensional analysis of a three-dimensional phenomenon}

As mentioned in the introduction, three-dimensional boundary layers have a third component profile, the $w$-profile, tangent to the body surface and transverse to the streamwise direction. This component is often referred to as the cross-flow component of the boundary layer. The cross-flow component certainly exists over the surface of an undulatory swimmer in light of the three-dimensionality of their bodies and locomotory movements. Occasional difficulties in matching the particles of an image pair, especially at the trough phase of the body surface, suggested cross-flow and possibly transverse separation. Wolfgang et al. (1999) present numerical evidence that flow over the majority of a laterally compressed fish is highly two-dimensional. Three-dimensional 
effects become important along the dorsal and ventral edges. Three-dimensional flow, however, is not as important to the determination of streamwise skin friction, since it is the tangential profile in the streamwise direction that determines the streamwise component of wall shear stress. Wall shear stress due to cross-flows does not contribute to the rearward friction drag, but they do have the potential to affect swimming performance in a variety of ways. First, in all undulatory locomotion, wall shear stress associated with cross-flow would resist transverse motions of the body, stealing energy from the muscles. This is in addition to any form drag or induced drag due to possible transverse separation of the boundary layer as cross-flows move around the oscillating body segments. Second, the distribution of cross-flow wall shear stress over the animal could result in a net force in the cross-stream direction-dorso-ventral for fish and lateral for cetaceans. This effect would not be expected to occur in cetaceans owing to symmetry with respect to the plane in which undulatory motion takes place.

\subsubsection{Power to overcome friction drag}

Friction drag was used to estimate minimum power output during swimming. Our calculation of power per muscle mass necessary to overcome friction drag for a scup swimming $30 \mathrm{~cm} \mathrm{~s}^{-1}$ at a temperature of $23 \mathrm{C}$ is $0.6 \mathrm{~W} / \mathrm{kg}$ (Table 3.1). This is based on red ('slow') muscle mass, using the value $2.09 \%$ for red muscle mass to body mass as determined by Zhang et al. (1996). Swank and Rome (2000) and Rome et al. (1992) found that scup, of similar size to those used here, primarily use red muscle for undulatory propulsion at speeds lower than about $80 \mathrm{~cm} \mathrm{~s}^{-1}$ at $20 \mathrm{C}$. Swank and Rome (2001) and Rome et al. (2000) drove excised scup red muscle at in vivo strains and stimulation patterns and measured power to range from approximately 1 to $14 \mathrm{~W} / \mathrm{kg}$ along the body $(x / L=0.3-0.7)$ at $10 \mathrm{C}$ for a swimming speed of $30 \mathrm{~cm} \mathrm{~s}^{-1}$. The shapes of the power distributions they report and the distribution of red muscle mass determined by Zhang et al. (1996) suggest that the average of the power distribution is a safe lower bound for available power per unit mass. This works out to between $3-8 \mathrm{~W} / \mathrm{kg}$, and 
suggests that our calculated power requirement to overcome friction drag at $23 \mathrm{C}$ and 30 $\mathrm{cm} \mathrm{s}^{-1}(0.6 \mathrm{~W} / \mathrm{kg})$, is only a fraction of the available muscle power. Furthermore, Rome and Swank (1992) observed maximum power output by scup red muscle to more than double as temperature increased from 10 to $20 \mathrm{C}$. Power at in vivo conditions at $20 \mathrm{C}$ for a swimming speed of $80 \mathrm{~cm} \mathrm{~s}^{-1}$ ranged from $4.4-24.3 \mathrm{~W} / \mathrm{kg}$ over the same region of the $\operatorname{scup}(x / L=0.3-0.7)$ (Rome et al., 1993). This gives a lower bound average of about 12 $\mathrm{W} / \mathrm{kg}$. It should be noted that at $30 \mathrm{~cm} \mathrm{~s}^{-1}$ scup tend to include occasional pectoral fin strokes in their swimming pattern. This decreases overall power requirements of the red muscle used for undulatory propulsion, thus the calculated power requirement per muscle mass that we report is higher than the actual value under these conditions.

Higher speeds result in higher power requirements. The power to overcome friction increases as $U^{3}$. Maximum swimming speed observed in scup was about 100 $\mathrm{cm} / \mathrm{s}$. This suggests power required may be as high as $22 \mathrm{~W} / \mathrm{kg}$. Optimized oscillatory power measured by Rome et al. (2000) was $31 \mathrm{~W} / \mathrm{kg}$ for scup at $20 \mathrm{C}$, but this value is achieved using contraction frequencies different from what occurs in vivo. This is the likely explanation for why scup do not swim using red muscle alone above about $80 \mathrm{~cm} \mathrm{~s}$ ${ }^{1}$ at $20 \mathrm{C}$. This is in agreement with our observation that scup could not swim for more than a few minutes at $100 \mathrm{~cm} \mathrm{~s}^{-1}$ suggesting, instead, that they are recruiting white ('fast') muscle, which functions anaerobically. White muscle makes up $51 \%$ of the scup body mass (Zhang et al., 1996) and tends to have a higher maximum power output (Altringham, 1994). Thus available power is not an issue on the short haul. We observed that sustainable speeds in scup max out closer to $60 \mathrm{~cm} \mathrm{~s}^{-1}$. At this speed, the $U^{3}$ effect would only predict a power requirement of about $5 \mathrm{~W} / \mathrm{kg}$--well within the expected range for red muscle at $23 \mathrm{C}$ based on the discussion above.

It should be mentioned here that friction drag is only part of the total hydrodynamic drag acting on the scup and the power required to overcome friction drag should be, indeed, only a fraction of muscle output capabilities. Of course, before the 
power required to overcome total drag can be calculated, flow separation and induced drag must be more thoroughly researched, and friction from flow over fins and through gills should be considered (Webb, 1975).

Available muscle power in the smooth dogfish swimming $20 \mathrm{~cm} \mathrm{~s}^{-1}$ (Table 3.1) was estimated using (1) the power for scup red muscle, (2) the distribution of red muscle mass for scup (Zhang et al., 1996), and (3) the percent of red muscle in a steak section of a spiny dogfish (Squalus acanthias) at $x /=0.67$ (Greer-Walker and Pull, 1975). GreerWalker and Pull (1975) report that $14.3 \%$ of the total muscle mass at $x / L=0.67$ in spiny dogfish is red muscle, whereas the value is about $9 \%$ in scup (Zhang et al., 1996). This results in an estimate of red muscle per body mass in spiny dogfish of about $3.3 \% \mathrm{vs.}$ $2.09 \%$ in scup as reported by Zhang et al. (1996). This results in a power requirement of just $0.2 \mathrm{~W} / \mathrm{kg}$. As for the scup, the estimates predict that there is plenty of muscle power available to overcome friction drag, even if $3.3 \%$ is a significant overestimate. Based on the $U^{3}$ effect on power required to overcome friction with increased speed, the predicted power requirement at $60 \mathrm{~cm} \mathrm{~s}^{-1}$ for dogfish at $23 \mathrm{C}$ is $5.4 \mathrm{~W} / \mathrm{kg}$.

\subsubsection{The advantages of boundary layer visualization}

The analysis of drag, thrust, power, and pressure distribution from the measurements of the flow around a swimming fish is an attractive alternative to theoretical hydrodynamic models. The application of existing hydrodynamic models to real fish shapes is limited, and it is difficult to incorporate the effects of complex locomotory patterns. Not only do experimental studies avoid such difficulties, but they are also necessary to validate existing theory. In this way, high-resolution flow visualization, which has enabled us to quantify flow as close as $0.1 \mathrm{~mm}$ from the body of a swimming fish, promises a new perspective on the mechanisms of undulatory locomotion and opens a door to much needed comparative studies. 

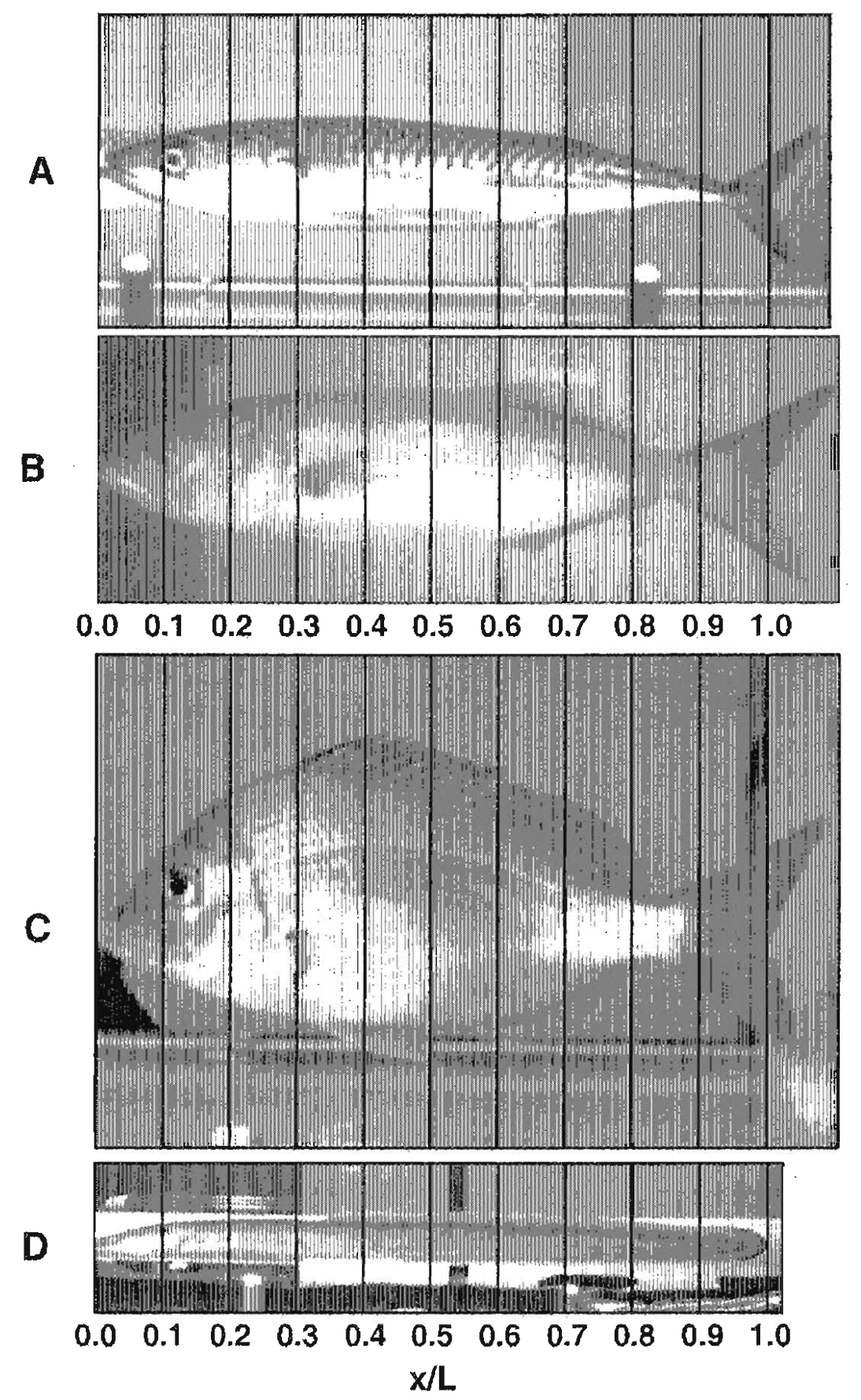

Fig. 4.1 Images of representative specimens scaled by length for comparison of body shape and structure: (A) mackerel, (B) bluefish, (C) scup, and (D) eel. Scale is shown twice to facilitate comparison. Note that length, $L$, in this investigation is measured from the snout to the fork of the tail, so called 'fork length'. 


\section{Chapter 4}

\section{Advances in data acquisition}

The preliminary investigation of the fish boundary layer was limited in scope due to the difficulties of acquiring and analyzing images of the flow. In order to resolve the boundary layer, a field of view on the order of $1 \mathrm{~cm} \times 1 \mathrm{~cm}$ was necessary. Based on the size of the test section used, there was roughly 1 in 4000 chance of the fish swimming through the field of view at any given second, and not all images acquired were usable data. Certainly, nothing in the way of rigorous ensemble averaging of data from various body positions and body phase were possible, except in a few unusual circumstances. Limits on the number of images that could be acquired and stored in a given amount of time further limited the ability to get sufficient data. These problems also made a larger comparative study more difficult. The following sections describe in detail a highly automated system developed by the author to acquire and efficiently reduce large quantities of boundary layer data on freely swimming fish. The system is a powerful tool for any work involving flow visualization around moving subjects or for experiments involving precision movements of the field of view in three dimensions.

\subsection{Specimens and trials}

Four species of fish were studied: (1) American eel, Anguilla rostrata, (2) scup, Stenotomus chrysops, (3) bluefish, Pomatomus saltatrix, and (4) Atlantic mackerel, Scomber scombrus. Fig. 4.1 shows representative sideview images of each fish species scaled by length, $L$, to facilitate comparison of body shapes and structures. Table 4.1 summarizes all of the experimental trials and significant experimental variables including numbers of each species used, and average fish lengths and masses. A total of 24 live 
Table 4.1: Summary of experimental trials

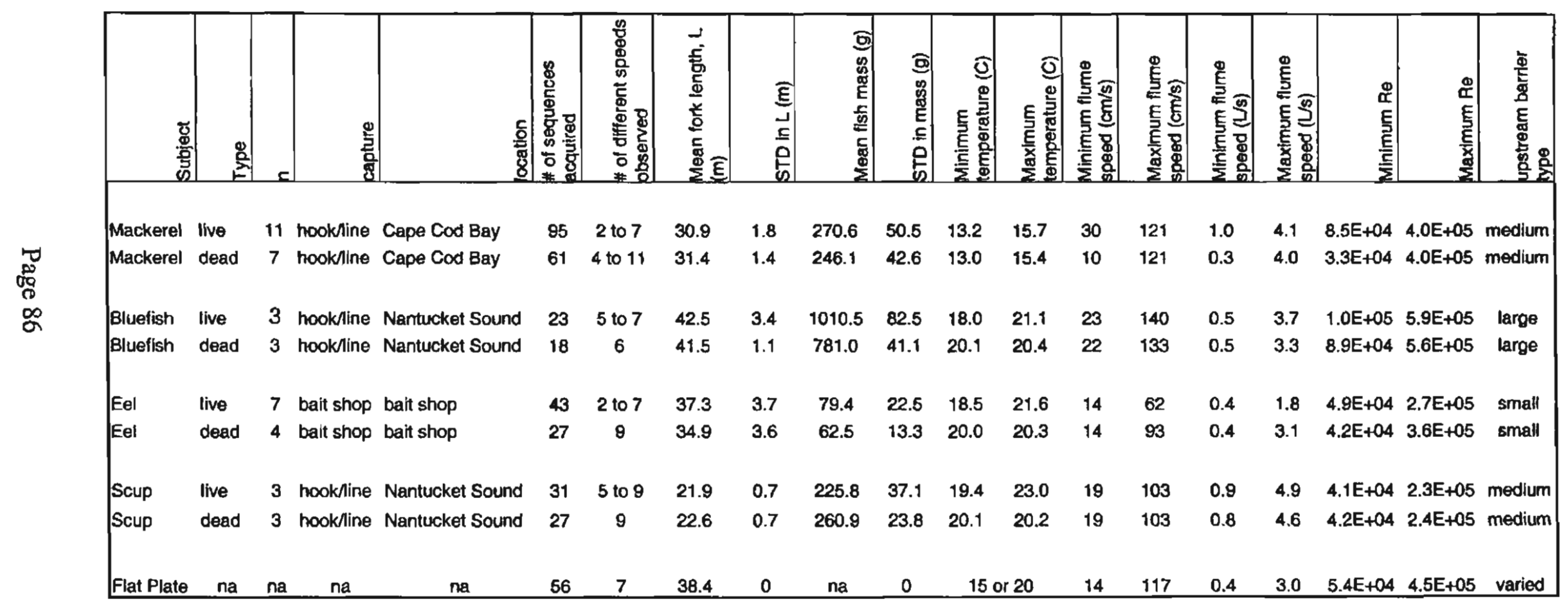


specimens were studied in 192 swimming sequences lasting 5 to $15 \mathrm{~min}$. This represents 4,500-13,500 image pairs per sequence of which $10 \%$ or more generally resulted in usable data, totaling about 120,000 boundary layer realizations. In addition, 17 euthanized specimens were studied in 133 sequences in which the fish were mounted 'stretched-straight' in the flume. These trials will be referred to throughout this thesis as the 'rigid-body case' and will be given special attention in Chapter 6. Of the 17 rigidbody experiments, the majority of the eel, scup and mackerel specimens were individuals that had been used in the live swimming trials. In the rigid-body case, the boundary layer was imaged at 10 to 15 positions along the centerline of the fish taking 150 images at each position at several different flume speeds. This represents a sampling time of $5 \mathrm{sec}$, which is expected to be longer than the periods of any fluctuations in the flow. Approximately 17,000 sample boundary layer realizations out of a potential 130,000 were analyzed for the rigid-body case. Finally, 56 sequences of the flow over a flat plate aligned parallel to the flow were performed representing various conditions equivalent to those of the fish experiments. The boundary layer was imaged at 14 positions taking 150 images at each position producing about 60,000 image pairs, from which over 12,000 sample boundary layer realizations were analyzed. Recall that in preliminary experiments on scup, Stenotomus chrysops, and smooth dogfish, Mustelus canis (Chapter $3)$, only 270 boundary layer realizations were processed. The dramatic increase in boundary layer realizations presented in this thesis compared to the preliminary work is testimony to the significant advancements in the automation of boundary layer data acquisition and analysis described in this chapter and in Chapter 5.

\subsection{Specimen collection and care}

Bluefish and scup were collected by hook and line in Nantucket Sound, MA, USA. Mackerel were collected by hook and line in Cape Cod Bay, MA, USA. Eel were purchased from a local retailer in Woods Hole, MA, USA. The animals were kept in round 1000-liter holding tanks with a constant flow of fresh seawater from Nantucket 
Sound. All fish kept longer than 2 days were fed a bi-weekly diet of frozen squid. Fish were transferred to and from their tanks in 30-liter buckets or 60-liter coolers. Only fish that appeared to be in the best overall condition after collection and transportation to the holding tanks were selected for experimental trials. Fish were transferred to a flume for experiments in large plastic bags containing seawater to prevent bodily injury in the transfer process. Temperature in the holding tanks, transfer bags and flume were within 1 $\mathrm{C}$ of each other. Following live swimming experiments, fish were euthanized in a seawater bath containing the standard lethal dose of MS-222 (400 mg/L) in accordance with the WHOI LACUC protocol at the time of the experiments. The same procedure was used to euthanize additional fish for rigid-body experiments.

\subsection{Flume test section}

Experiments were performed in a temperature-controlled recirculating flume capable of producing flow speeds up to $2 \mathrm{~m} / \mathrm{s}$ (Fig. 4.2, Engineering Laboratory Design, Inc.). Experimental temperatures and swimming speeds for each fish species studied are given in Table 4.1. In the case of bluefish, scup and mackerel, the temperatures reflect the temperature of the bodies of water from which the fish were caught at time of capture. Eel were kept and studied at the local temperature of Nantucket Sound. The flume test section (Fig. 4.3) was constructed entirely of Plexiglas and measured $170 \mathrm{~cm} \mathrm{x} 45 \mathrm{~cm} \mathrm{x}$ $45 \mathrm{~cm}$. The transparent flume bottom was especially useful for boundary layer visualization. Cameras could be mounted below the flume and therefore did not interfere with the flume lid or work within the flume test section. Position in the flume was defined according to a Cartesian coordinate system, where ' $X$ ' was the horizontal, streamwise direction, ' $Y$ ' was the horizontal, 'crossflow' direction, positive toward the backside of the channel, and ' $Z$ ' was the height from the bottom. The test section was preceded by a significant contraction (6:1 area ratio) to encourage smooth flow in the test section. 


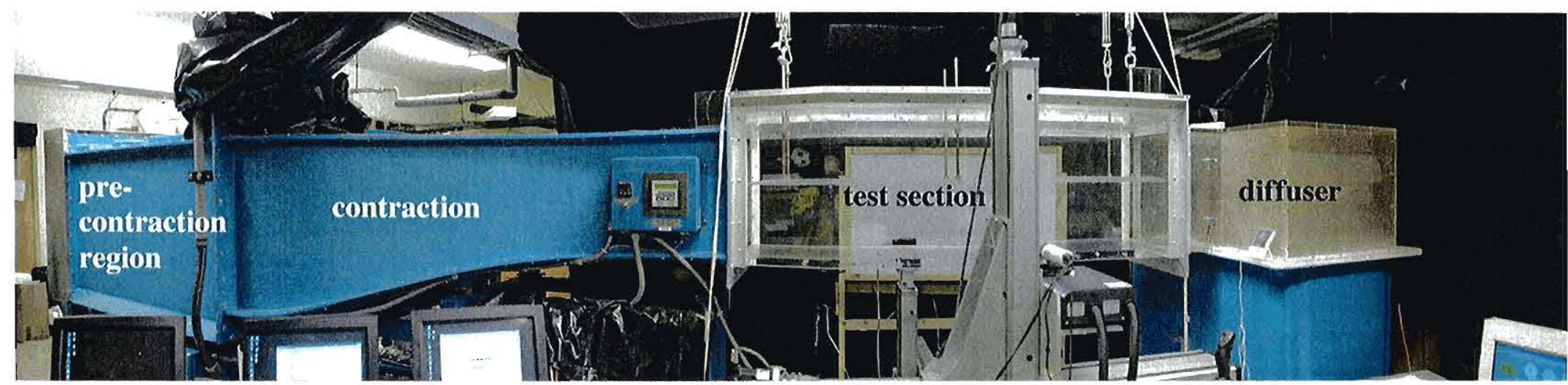

Fig. 4.2 Panoramic image of the flume (Engineering Laboratory Design, Inc.) used for live fish, rigid-body and flat plate experiments. The Plexiglas test section $(170 \times 45 \times 45 \mathrm{~cm})$ sits between a $6: 1$ contraction and a Plexiglas diffuser. A motor driven impeller draws water from the diffuser to a forward reservoir through a 12 " diameter PVC pipe running along the floor (not visible). Flow passes through a heat exchanger and flow straightener into the pre-contraction region. Maximum speed in the test section is about $2 \mathrm{~m} / \mathrm{s}$. Turbulence intensity claimed: $1 \%$. Plug profile variation claimed: $1 \%$. See Chapter 6 for a complete characterization of flow in the flume. 


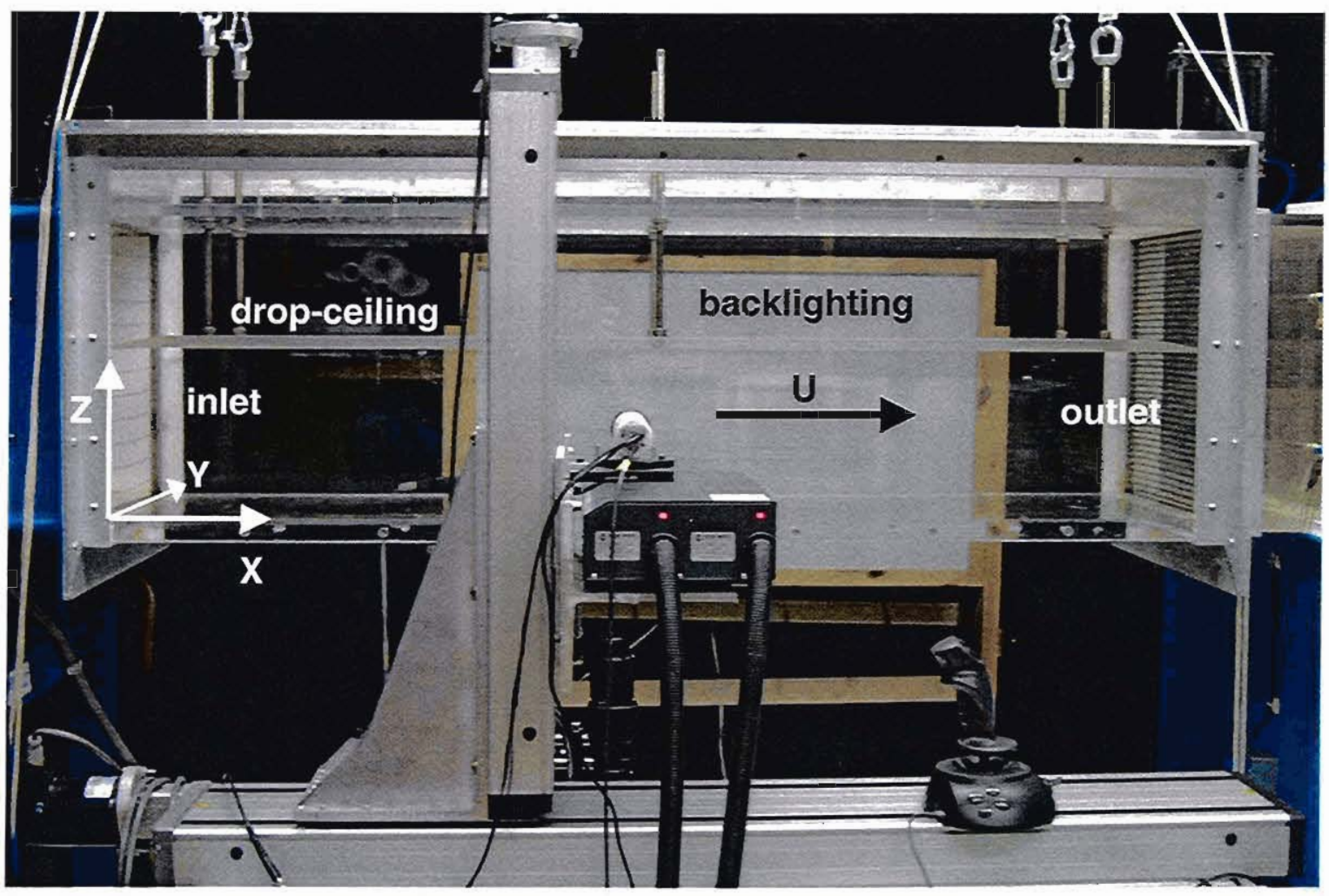

Fig. 4.3 Image of the flume test section $(170 \times 45 \times 45 \mathrm{~cm})$. Flow is from left to right. A fine stranded grid is in place at the inlet and a more substantial, mesh barrier is in place at the outlet. The acrylic 'drop-ceiling' suspended by threaded rod is visible. In the foreground is a two axis robot that carries the laser head and the sideview camera. In the background is a semi-opaque, white acrylic sheet that was illuminated from behind providing uniform backlighting to produce a silhouette of the fish in the sideview camera. The $X Y Z$ coordinate system is shown. $Y$ points into the page. 
In general, the test section was used as an open channel, $45 \mathrm{~cm}$ deep, but an acrylic 'drop-ceiling' (Fig. 4.3) was sometimes lowered onto the surface to prevent fish from jumping out of the flume, or into the flow to decrease the flume depth to $25 \mathrm{~cm}$. The ceiling leading and trailing edges were machined to $10^{\circ} \mathrm{knife}$ edges to reduce impact on the flow in the test section when lowered into the flow. In the streamwise direction, fish were constrained to swim in the test section using barriers at the inlet and outlet constructed from plastic netting. The netting used in the inlet barrier was a rectangular mesh of thread-like strands known as bird-netting (Aquatic Eco-Systems, Inc.). Three different mesh sizes were used, which will be referred to in this thesis as small, medium and large. The actual mesh sizes were $1.3 \mathrm{~cm} \times 1.3 \mathrm{~cm}$ (small), $1.6 \mathrm{~cm} \times 1.7 \mathrm{~cm}$ (medium), and $3.0 \mathrm{~cm} \times 3.7 \mathrm{~cm}$ (large). The largest possible mesh size was used for each fish species (Table 4.1). The downstream barrier was made of $1.3 \mathrm{~cm}$ square mesh netting with a heavier strand than the inlet barrier to prevent fish from breaking out of the test section and being swept downstream.

Flow in the flume test section was measured with no fish present for each inlet barrier type at three speeds $(23 \mathrm{~cm} / \mathrm{s}, 58 \mathrm{~cm} / \mathrm{s}$ and $98 \mathrm{~cm} / \mathrm{s}$ ), two temperatures $(15 \mathrm{C}$ and $20 \mathrm{C}$ ), and both open and closed channel arrangements. In the closed channel case, the ceiling plate was lowered to $Z=25 \mathrm{~cm}$. The case of no inlet barrier was also observed for comparison. The flow was visualized at $45-50$ positions in each of 3 vertical crosssections of the test section at $X=30 \mathrm{~cm}, 87 \mathrm{~cm}$ and $144 \mathrm{~cm}$. Wall boundary layer thickness, turbulence intensity, uniformity of the plug flow, and the impact of the inlet barriers were examined and are treated thoroughly in Chapter 6.

\subsection{Strobe imaging of the flow}

In general, fluid flow around the fish was visualized in the same way as in the preliminary experiments (section 3.1.3). Particles in the flow were illuminated by a horizontal, pulsed laser sheet (thickness $=1 \mathrm{~mm}$ ) and imaged at right angles to the sheet. 
The most significant differences in the flow visualization in the automated system were the use of an additional high-resolution digital video camera (Roper ES 1.0, 1008 pixels $\mathrm{x}$ 1018 pixels), a different type of seeding particle, and a streaming video acquisition system. The additional camera was used to acquire a wide-angle view $(14 \mathrm{~cm} \mathrm{x} 14 \mathrm{~cm})$ of the flow fieid and determine the location of the body surface of the fish. This camera will be referred to as the 'nearfield camera' (Fig. 3.1). In the preliminary experiments, only two cameras were used, the 'boundary layer camera' (Roper ES 1,0, 1008 pixels $x$ 1018 pixels), with its small field of view $(1.36 \mathrm{~cm} \times 1.36 \mathrm{~cm})$, and a 'sideview camera' (Texas Instruments, Multicam CCD, 752 pixels x 480 pixels) to locate the laser position on the fish. This made it difficult to determine the phase and amplitude of the body wave at the time and position of each measurement. The nearfield camera field of view was positioned so that it contained the field of view of the boundary layer camera. A 17 $35 \mathrm{~mm}$ zoom lens (Nikon, Nikkor AF-S) fit with a c-mount-to-bayonet adaptor was used on the nearfield camera. The boundary layer camera was fit with a $105 \mathrm{~mm}$ macro lens (Micro-Nikkor AF). The sideview camera was fit with an $8.5 \mathrm{~mm}$ wide-angle television lens (Cosmicar). The nearfield view was also used to determine of the $Y$ position of the fish in the test section based on the width of the diverging laser sheet.

Silver coated hollow glass spheres with average diameter of $10 \mu \mathrm{m}$ (DANTEC; Potters Industries Inc.) were used as seeding particles. In preliminary experiments, fluorescent particles were used, however it was found that the filters used with fluorescent imaging often rendered the fish body surface too dim to locate automatically. The fluorescent particles were still visible with the filters removed, but excessive glare off the fish body surface, which also interferes with body edge detection, required the use of lower laser intensity or smaller camera apertures. However, this resulted in the dropout of dimmer particles from images, reducing the resolution of the boundary layer flow. The higher reflectance of silver coated particles increased the visibility of particles at lower laser intensities and solved the body surface illumination problems. 
Images from the boundary layer camera had a scale of 73.7 pixels $/ \mathrm{mm}$ or $13.6 \mu \mathrm{m}$ per pixel. Although the average physical particle diameter was smaller than a pixel, the actual particle images were slightly larger due to glare, aggregate particles and ambient particles in the seawater. This was desirable since it allowed for sub-pixel accuracy in particle tracking. If particles are imaged as a single pixel the location of that particle is unknown within that pixel, whereas if a particle spans $2-4$ pixels, a more precise particle centroid location can be determined by a Gaussian fit or center of intensity.

Timing for the laser and all three cameras was controlled by the same methods as in the preliminary experiments. The time delay, $\Delta t$, between laser pulses, i.e. between exposures of the flow, was set at $2.1-6.7 \mathrm{~ms}$ depending on flume speed. Simultaneous images from the three cameras were streamed directly to hard drive arrays on three separate PCs capable of up to $2 \mathrm{hrs}$ and $20 \mathrm{~min}$ of continuous acquisition. That is 250,000 images, or $250 \mathrm{~GB}$, for the high-resolution cameras. In general, acquisition was broken into $5-15$ min long sequences at particular swimming speeds. Streaming video acquisition was mediated by PCI image acquisition cards (National Instruments, NI PCI1424, NI PCI-1409) and code written by the author using retail subroutines (Visionstream 100) fashioned for LabVIEW. Each image of the video stream from each camera is assigned an image number, or time-code. Acquisition of the three video streams were initiated independently, therefore it was necessary to determine the offset between the time-codes of corresponding images from the three cameras. This was achieved by manually interrupting the laser trigger input at the start of acquisition, resulting in a series of dark images in each camera image stream. Code was written to find the first dark image of any image stream and automatically determine the time-code offsets needed to synchronize the streams from the three cameras. 


\subsection{Robotic control of data acquisition}

In preliminary experiments, the small field of view of the boundary layer camera was fixed in the flume. The camera could be repositioned by manually sliding the camera along an optical rail that supported it, but this did not solve the problem of getting the fish to swim in the vicinity of the field of view. Fish tended to move to a position in the test section, remain there for $30 \mathrm{sec}-5 \mathrm{~min}$, and then move to another location. By the time the camera was moved and refocused, the fish would generally move to a new location. Additionally, fish were usually spooked during this repositioning procedure. It became clear that in order to acquire acceptable amounts of data, remote control of camera position and focus was needed. This was achieved by mounting the three cameras and the laser on two synchronized robots that allowed image acquisition throughout the test section by joystick control (Microsoft Corporation, Sidewinder Joystick, USB). The sideview camera and the laser were mounted on one robot alongside the test section with movement in the $X$ and $Z$ directions (Fig. 4.4). The boundary layer and nearfield cameras were mounted beneath the test section on the second robot with movement in the $X, Y$ and $Z$ directions (Fig. 4.5).

Both robots were constructed with ball-screw, linear actuators (Techno-Isel, heavy duty slides, 4 carriages) with a pitch of $5 \mathrm{~mm}$ per ball-screw revolution. The actuators were powered by stepper motors with a resolution of 20,000 steps per revolution, offering a potential resolution of $0.25 \mu \mathrm{m}$. Actuator speeds of $5-10 \mathrm{~cm} / \mathrm{s}$ could be achieved, but speeds of $1-5 \mathrm{~cm} / \mathrm{s}$ proved to be sufficient. Slower speeds are preferable to reduce transients in robot structural vibration due to starting and stopping. Stepper motors were powered by manufacturer specified motor amplifiers (Industrial Devices Corporation, Nextstep line). Joystick input and stepper control output was orchestrated by code written by the author within the graphical programming language LabVIEW 6.1 (National Instruments) and 2 National Instruments, Flexmotion PCI-7344 motion control cards (break-out box, UMI-7764). Each motion controller can 


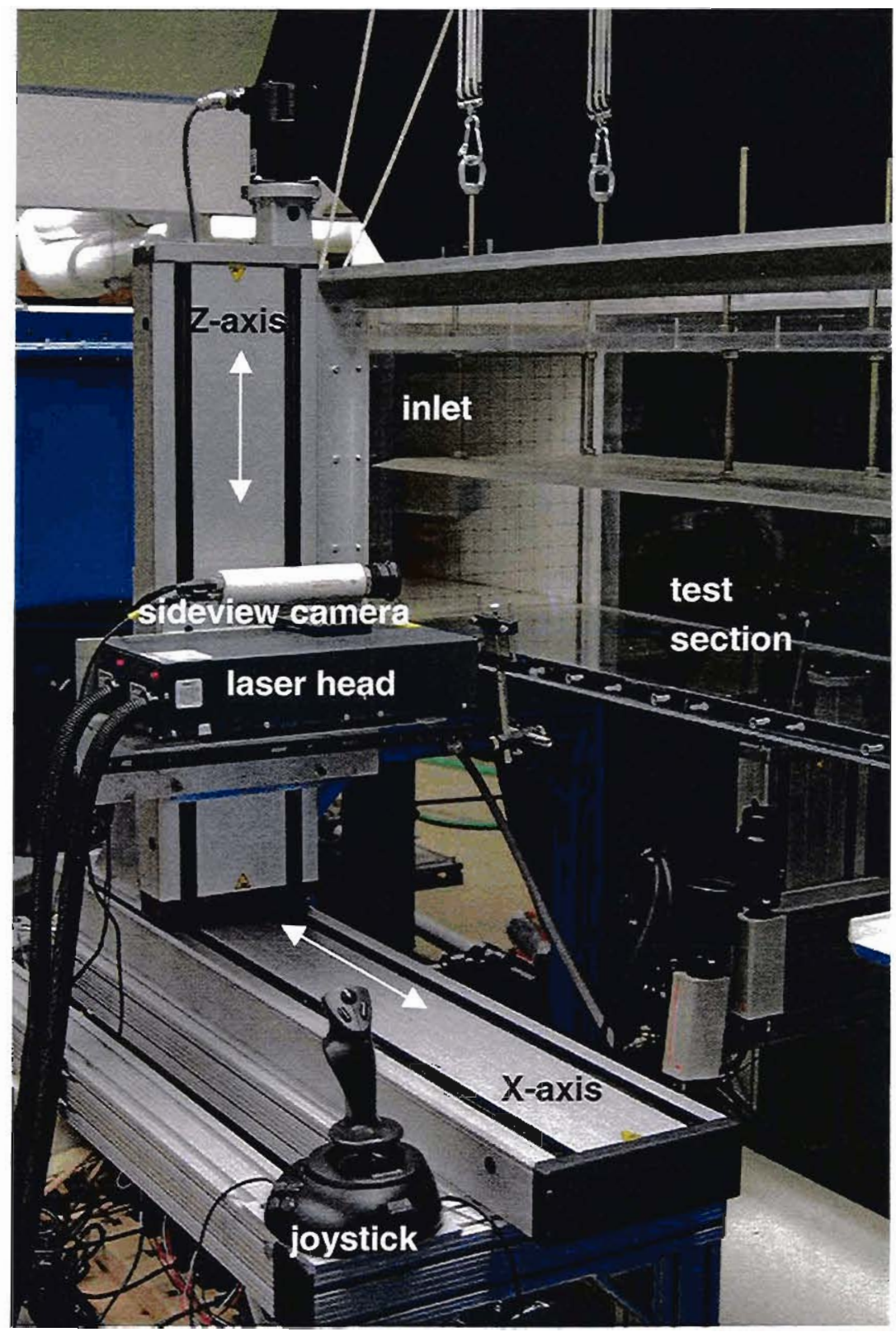

Fig. 4.4 Two axis robot and joystick used to position the laser and sideview camera. The robot is seated on a support table constructed from $80 / 20$ brand aluminum beams. The nearfield and boundary layer cameras can be seen under the test section. The large grid inlet barrier and the 'drop-ceiling' plate can be seen, as well. 


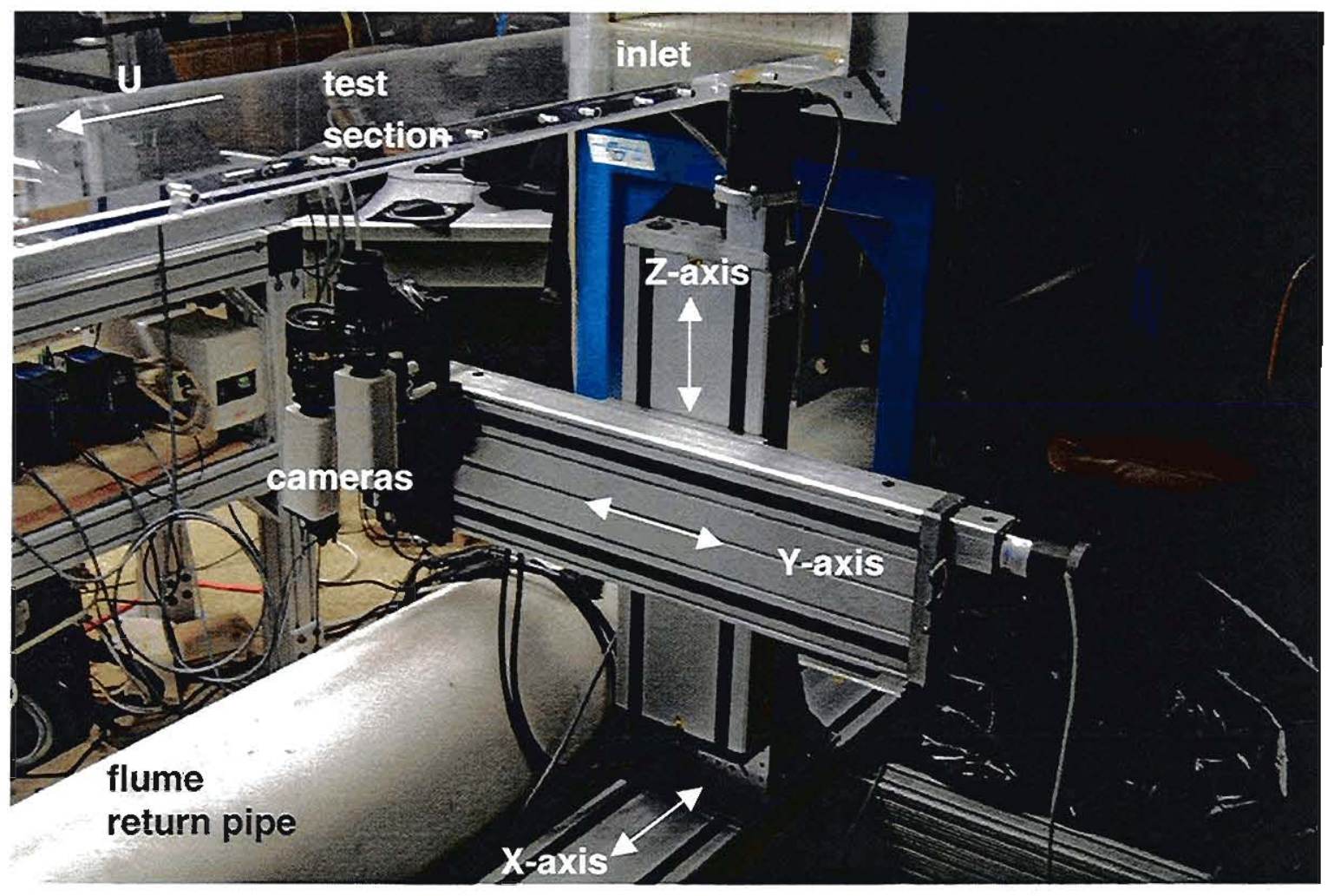

Fig. 4.5 The three-axis robot use to position the boundary layer (right) and nearfield (left) cameras. The robot was seated on base of $80 / 20$ brand aluminum beams on the opposite side of the test section from the $X Z$ robot. 
simultaneously control 4-axes of motion. The motion control toolbox of LabVIEW includes subroutines for the general control of stepper motor amplifiers. Input subroutines for the Sidewinder joystick were found on the developer resource pages of the National Instruments website (http://www.ni.com). The code written by the author allowed for smooth, simultaneous or independent jogging of all five robot actuators, as well as precise movements of $1 \mathrm{~cm}, 1 \mathrm{~mm}$, or $0.1 \mathrm{~mm}$ for positioning and focusing when needed. The code links all jogging and stepping functions to the joystick for complete remote control of the robots. In addition, the code can memorize and repeat a program of moves. This feature was used to take measurements at precise positions on the rigid fish and flat plate, and throughout the entire test section at different flow speeds. The code also allows the motion controller cards to communicate with data acquisition boards (National Instruments, PCI-6024E) on the camera PCs to orchestrate the acquisition of a set number of images at any given position, if desired.

Most importantly, the robots were geared in software to automatically correct for the index of refraction of seawater in real time so that the boundary layer and nearfield cameras were constantly focused on the laser sheet. Optical distance in a medium is the real distance times the index of refraction, therefore the laser must move about 1.33 times the distance moved by the cameras in the $Z$ direction to stay in focus. The index of refraction was first measured using the robots independently, focusing the cameras at two different vertical positions of the laser. The ratio between $\Delta Z$ of the laser and $\Delta Z$ of the camera is the index of refraction. Conveniently, since focusing is achieved by motion of the cameras and not the helical focus of the lenses themselves, the field of view is preserved, spatial conversion factors (pixels $/ \mathrm{m}$ ) are constant, and both the boundary layer camera and the nearfield camera stay in focus on the same focal plane. 


\subsection{Automatic calibration}

The precision of motion afforded by the robots made for easy automatic calibration of image fields. Two triangles were cut from black electrical tape and affixed to a small panel (3" x 5") of opaque white 3/8" acrylic sheet. The triangles were cut so that they were approximately $1-3 \%$ of the area of the field of view of the boundary layer and nearfield cameras, respectively. The plastic panel was then placed face down on the bottom of the flume. The cameras were focused on the triangles and for one camera at a time the robot was moved so that the corresponding triangle was fully visible in each corner and in the middle of the field of view. An image was snapped at each position and the precise robot motion is recorded. Image analysis code written by the author in LabVIEW automatically finds the triangle in each image, calculates the change in pixel position by the triangle centroid and determines an average calibration factor in pixels per meter. The code also finds the small triangle in the nearfield camera field of view when it is positioned at the center of the boundary layer camera field of view. This is used to determine the precise location of the boundary layer camera field of view in the nearfield field of view. The calibration can be fully automated using a memorized program of calibration motions and having the robot signal the calibration image acquisition.

Calibration of the sideview camera image field was somewhat more complicated, but also automatic. Since the sideview camera was fixed in the $Y$-direction, the pixels per meter calibration factor changes depending on the location of the fish in the tank. A very short focal length lens was used, and the depth of focus was large enough that the fish stayed in good focus throughout the flume. The divergence of the laser sheet was used to in the calibration. The span of the laser sheet in pixels at the $Y$-location of the fish was determined automatically from the bright horizontal line seen on the fish in the sideview camera. This was performed for every sideview image of a particular swimming sequence. Simultaneously, the span of the laser sheet in meters was determined from the nearfield view by automatically locating the edges of the laser sheet and the body surface 
of the fish, and using the pixels per meter calibration of the nearfield view as detailed in the preceding paragraph. The span in pixels in the sideview camera divided by the real span in meters determined from the nearfield view is the pixels per meter calibration for the particular sideview image. Since the bright line on the fish is not always completely visible, or it is slightly shortened by fish curvature, the sideview calibration was refined by an additional step. The calibration factor vs. $Y$-position in the tank was determined by imaging a strip of $3 / 8$ " white plastic sheet $10 \mathrm{~cm}$ wide held vertical at several $Y$-positions in the tank. The predicted $Y$-position of the fish in each sideview image was then determined from the rough sideview pixels per meter calibration for each image. This $Y$ position was then plotted vs. the span of the laser in the nearfield view at the position of the fish. Since the edges of the laser sheet were straight, diverging lines, the span was directly proportional to $Y$-position in the tank. Therefore the plot should be a straight line, and all of the underestimated sideview spans should be scatter below that line. This was indeed found to be the case and the line gave the correct relationship between laser span in the nearfield view and position in the tank. Since the calibration factor vs. $Y$ position in the tank was precisely measured in the sideview with the $10 \mathrm{~cm}$ wide plastic strip, the $Y$-position determined from the nearfield view could be used to determine the accurate sideview calibration factor.

Of course, it would have been much easier to simply measure the span of the laser sheet at one known $Y$-position in the nearfield view. Then the determination of the laser span in the sideview and the construction of the $Y$-position vs. span in the nearfield view could be avoided. In some swimming sequences the front or back walls were sometimes visible in the nearfield view. The laser sheet span at these known $Y$-positions could have been used, but the sideview calibration by the approach outlined above proved to be exceptionally accurate. Determination of the position of data acquisition on the fish was within $1 \mathrm{~cm}$, smaller than the field of view of the boundary layer camera, and therefore smaller than the region sampled. 


\subsection{Automatic scanning of long video records for usable data}

Acquisition of sequences of thousands of images makes the acquisition of good data more likely, but it also makes locating that data after acquisition more difficult. A $5 \mathrm{~min}$ video sequence has 9000 images, which if scanned manually at about $1 \mathrm{~Hz}$ non-stop would take $2.5 \mathrm{hrs}$. To avoid this tedious process, automatic image scanning code was written by the author that was able to scan the data at $10-30 \mathrm{~Hz}$. The code simply calculates the average pixel intensity for selected pixel columns of successive images of the boundary layer camera and compares the average to sampled background intensity. If the average exceeds a user-defined intensity above background, then it is assumed that the fish surface is present in the image and the image time-code is noted. Later, the list of time-codes is used to extract the good images from the video stream file and write them as individual TIFF image files. The scanning code is very efficient at finding usable data and reduced the number of archived images to $10 \%$ of the original sequence on average. The list of good images was also used to extract the nearfield and sideview images that coincided with the boundary layer images using the offsets determined as described earlier. A user determined number of images before and after each good image is extracted from all three video streams so that body movement and nearfield flow associated with to each boundary layer realization can be determined.

The writing of TIFF files from video stream files runs at a rate of about $5-10 \mathrm{~Hz}$, but since the data has been reduced 10-fold, the apparent speed is $50-100 \mathrm{~Hz}$. Image data was archived on CD-ROM and/or external hard drives (Interactive Media Corporation, Kanguru Quicksilver, 120 GB, USB 2.0). 
Page 101
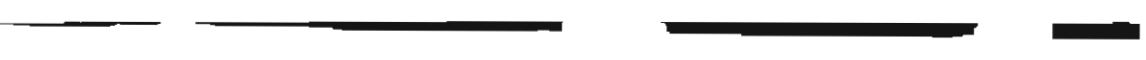


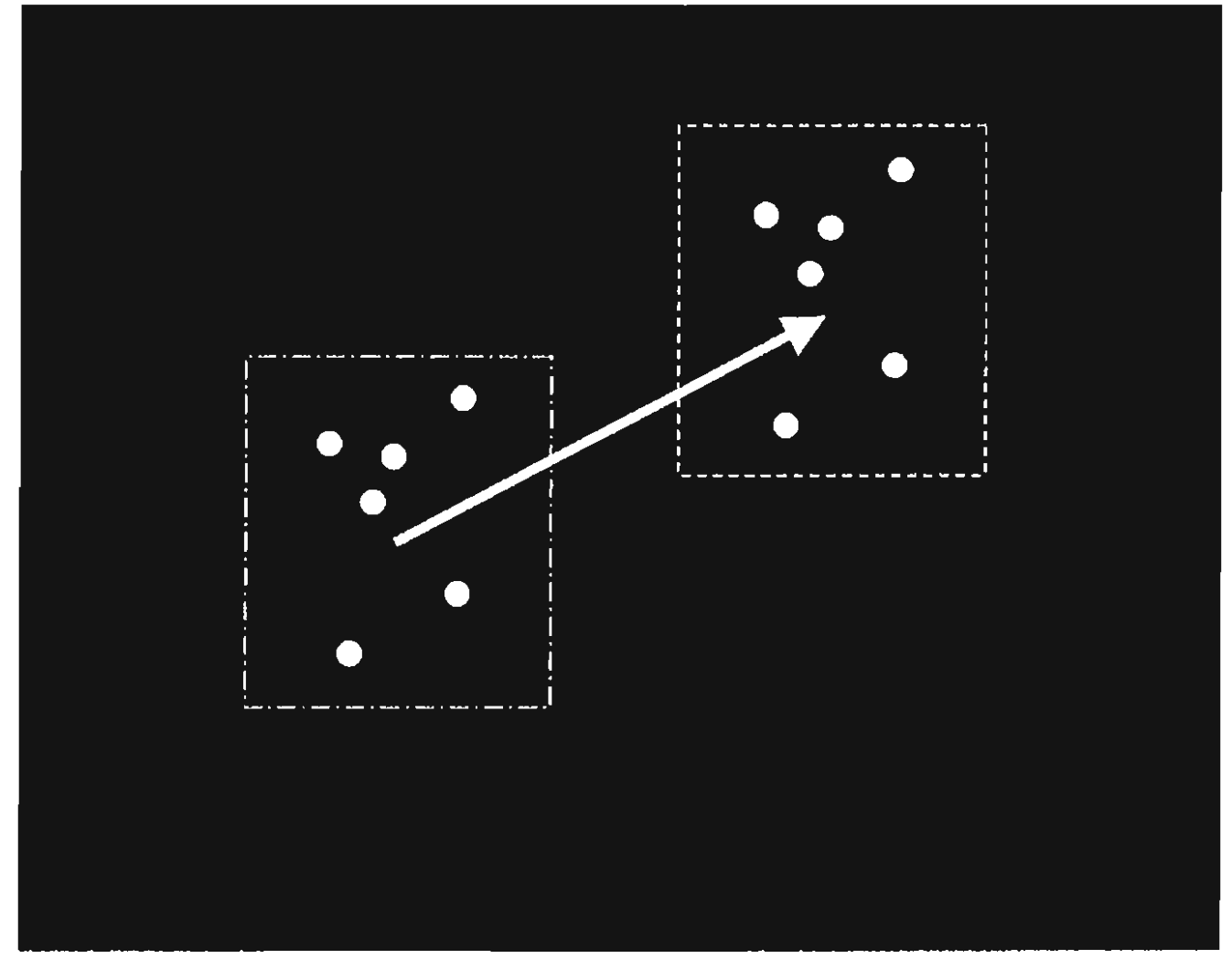

Fig. 5.1 An illustration of digital particle imaging velocimetry. The sub-window (dashed-dot) defined in image 1 moves to another position (dashed) in image 2. Twodimensional cross-correlation results in a peak when the orginal sub-window is placed over the location of the sub-window in image 2. 


\section{Chapter 5}

\section{Automatic boundary layer PTV and analysis}

Chapter 4 described the solutions to the difficulties of data acquisition in profiling the fish boundary layer. Nevertheless, the greatest difficulty in the preliminary experiments was processing the image data. Conventional particle tracking codes failed and particle matching between image pairs was preformed manually, particle by particle. This was time-intensive, tedious, and required significant sub-sampling of the data. Furthermore, the analysis of tens of thousands of boundary layer profiles required the development of automatic analysis code, as well. The following discussion describes the fully automatic particle tracking and profile analysis code developed in this investigation.

\subsection{The failure of conventional DPIV and DPTV to resolve the boundary layer}

In general, conventional DPIV code fails to resolve steep velocity gradients such as those very close to the surfaces of objects in a flow. This is due to the shape and size of interrogation regions, of sub-windows, of the flow images that it uses to measure velocity. In conventional DPIV, the first image (image 1) in a pair of sequential flow images is divided into a grid of rectangular sub-windows. The algorithm searches for the new location of the image pattern of each sub-window in the second image (image 2) of the image pair (Fig. 5.1). It does this by looking for a peak in the 2-D cross-correlation of each sub-window in image 1 with sub-windows of the same size in image 2. Therefore only one flow velocity for each sub-window is determined and the resolution of velocity gradients in the flow is limited by sub-window size. Sub-window size cannot be reduced arbitrarily. The sub-window must contain a sufficient number of particles for accurate cross-correlation. 
Increased seeding density and higher resolution can improve the resolution of velocity gradients by conventional DPIV to some degree, however, if one attempts to resolve a very steep velocity gradient very close to a moving object surface added difficulties arise. The body and body glare affect the cross-correlations strongly. Subwindows shaped parallel to the body surface would be better suited than grid-squares parallel to the image axes, and even then, resolution of steep velocity gradients would require the sub-windows to be thin. Conventional DPIV does not incorporate edge finding or custom shaped grids, and even with these components built in, the problems of particle density and grid size could cause problems in resolving the fish boundary layer.

Conventional digital particle tracking velocimetry, DPTV, also fails as an acceptable technique for measuring steep velocity gradients as found in the fish boundary layer. In the case of conventional DPTV, the user generally defines an acceptable range of distance and angle for the motion of particles from one image to the next (Fig. 5.2). However, in the boundary layer, velocities range from 0 at the body surface to approximately the freestream velocity, $U$. Therefore, for standard DPTV to work in the boundary layer, it would be necessary: (1) to define the acceptable range of particle motion at several distances from the body surface, (2) define the range of travel angle as very narrow, and/or (3) have a particle density such that the distance between particles is less than the distance that a particle travels in the freestream. These constraints either require the user to have a lot of information about the flow already, or to have a particle density that may not be able to provide enough information about the velocity gradient. In the former case, gathering the necessary information about the flow would cease to make the particle tracking technique automatic and greatly increase processing time.

In this investigation, a fully automatic boundary layer profiling code has been developed that requires the user to know essentially nothing about the flow in the boundary layer and can track particles in steep velocity gradients at particle densities ideal for resolving those gradients. Since the code is fully automatic, processing time for 


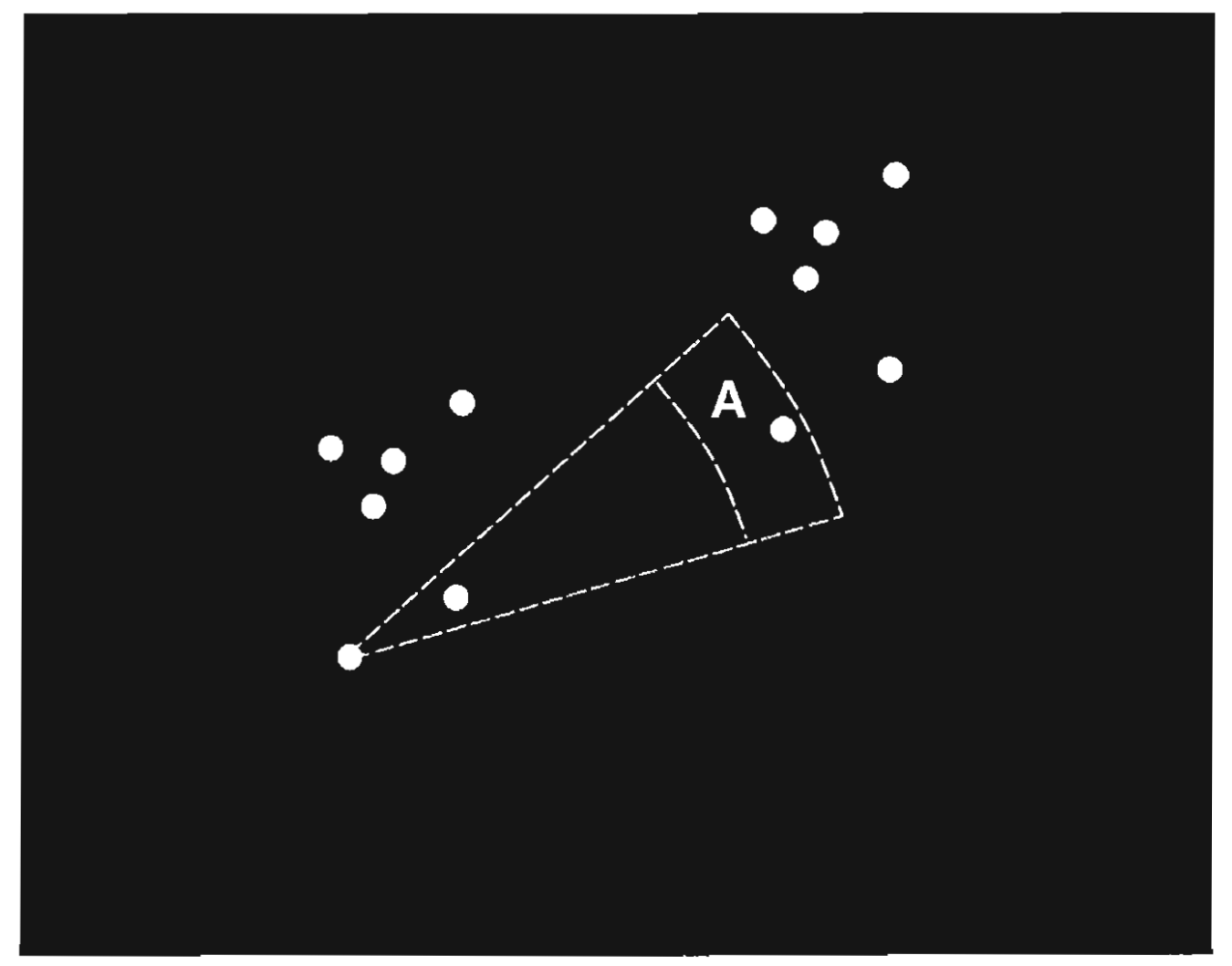

Fig. 5.2 An illustration of conventional digital particle tracking velocimetry. Particles in image 1 are allowed to move a prescribed distance and angular range (A). Any particle landing in this range in image 2 is considered a potential match. 
a single image pair has been reduced dramatically. The particle tracking module of the code can determine the tracks of 825 particles in $14 \mathrm{sec}$. This is approximately 12,000 times faster than manual particle tracking. The entire boundary layer visualization process requires an average of $2 \mathrm{~min} 10 \mathrm{sec}$ to take a raw image pair, locate the body surface, track the motion of the body surface, remove glare, locate and centroid particles, match particles, calculate the boundary layer profiles and plot them. This is about 150 to 300 times faster than the manual and semi-automatic methods used in the preliminary investigation. Assuming that the same number of particles were tracked, it would take 5 to 10 hours to process one image pair manually. Even though only $1 / 10$ to $1 / 20$ as many particles were tracked per image pair in the preliminary investigation, it still took more than 130 hrs of tedious manual particle matching spread out over several months to produce just 270 usable boundary layer profiles. Since the new code is automatic, multiple PCs can be utilized simultaneously around the clock to multiply the data processing rate. Six to eight PCs running constantly processed an average of 4500 boundary layer realizations per day.

\subsection{An automatic boundary layer profiling and analysis code}

The boundary layer profiling code developed by the author has several stages: (1) object surface edge detection, (2) surface tracking, (3) surface and glare removal, (4) particle centroiding, (5) particle tracking, (6) boundary layer profile calculation, and (7) boundary layer profile analysis. The particle tracking algorithm, which was independently developed by the author, was found to be similar to algorithms developed earlier by Kim and Chen (1992) and Wernet (1993) for particle tracking in general flows, however significant differences and advances exist. In particular, the mathematical particle tracking problem is itself transformed into an image processing problem, which simplifies the process. Moreover, the algorithm developed in this thesis was custom designed to resolve boundary layer flow and nearfield flow over surfaces. See Udrea, et al. (2000) for a thorough review of particle tracking methods. 


\subsubsection{Automatic object surface edge detection}

Boundary layer profiles are constructed using coordinate systems fixed on a body surface. Therefore, the location, shape and movement of the surface must be known accurately. The surfaces of fish and the flat plate in the fields of view of the nearfield and boundary layer cameras were located automatically by searching for the widest peak in pixel intensity in the pixel columns of the images most nearly perpendicular to the surfaces, i.e. the $Y$-direction pixel columns in this investigation. These pixel columns are simply cross-sections of the image. The plot of pixel intensity along each pixel column revealed the bright object surface to be a steep-walled plateau, typically on the order of 100 pixels wide. By contrast, particles appeared as sharp peaks typically 5-10 pixels wide at their base. Therefore, it was easy to distinguish between the surface 'plateau' and particle peaks. Once the surface plateau was located, a pointer was moved along the fluid facing slope leading up to the plateau until it reached the top edge. The top edge was determined by taking the position at which the fluid facing slope decreased to 0.2 , or where the pixel intensity reached the maximum 255 (8-bit image). This process was repeated for each pixel column of the image. The pixel columns and the locations of the surface plateau edges represent the $X$ and $Y$ positions of the object surface, $S(X, Y)$. In the case of each image, $X$ and $Y$ were determined in pixels with respect to the image frame, but the axes correspond in direction to the $X$ and $Y$ coordinates in the flume.

After the surface, $S(X, Y)$, in each image was located, it was filtered for errant peaks and gaps and smoothed with a triangular low pass filter to remove digital noise caused by the plateau edge finding technique. The width of the low pass filter was chosen carefully so as not to smooth out actual small-scale structures on the surface. 


\subsubsection{Surface tracking}

The precise motion of surfaces, $d S(X, Y) / d t$, for consecutive images was determined by rotating and translating the surface in the first image until the sum of the squared differences in the distances between its points and the points of the surface in the second image was minimized. Stated simply, the best least-squares fit of the surface in image 1 to the surface in image 2 was performed. Extrapolation between the points of each surface was performed so that motions of the surfaces, in theory, could be determined to 0.1 pixel accuracy. Code testing with artificial surface motions confirmed accuracy to $0.1-0.3$ pixels. This corresponds to errors in surface motion velocities of 1 $-2 \mathrm{~mm} / \mathrm{s}$, generally less than $1 \%$ of the freestream flow, $U$. Any shift in profile velocities due to these errors merely shifts the entire profile and the true profile shape is preserved. This technique, in addition to determining a translation and rotation of the entire surface, determines the translation of each surface point. Translation and rotation alone does not give that information unless the center of rotation is known. This allows for the true time-averaged surface to be determined for each image pair rather than simply averaging the corresponding surface positions in each $Y$-pixel column. The code outputs goodness of fit, and boundary layer data was only used for surface tracking that converged properly. This helped to filter out errant images that were classified as 'good' by the image scanning code described in section 4.7 .

\subsubsection{Surface and glare removal}

One of the problems in tracking particles automatically near a stationary or moving surface is coding the computer to distinguish between true particles, structures on the surface and glare from reflected light off the surface. The fish surface is easy to remove by setting all pixels on the fish side of the body surface to zero. Glare, on the other hand, is not quite as easy to remove since it varies with distance from the surface. If a simple image threshold is used, the threshold must be very high, otherwise the high 
glare near the surface remains. However, high thresholds degrade and even eliminate particle images further out in the flow and so are unacceptable. In this investigation, it was found that the combination of two custom low pass filters developed in this investigation could be used to precisely measure the glare at every location in the image. The glare was then subtracted from the original image and the surface was removed, leaving only the particles, essentially non-degraded.

These filters were applied to each pixel column of each image in the direction most perpendicular to the body surface (the $Y$-direction here). As mentioned earlier, particles appear as peaks 5-10 pixels wide in these image-intensity cross-sections. The first low pass filter was a simple running average with a bandwidth of 17 pixels, but before computing each average, the 5 highest intensity pixels were removed. This was applied far from the fish surface, where glare was low and increased very gradually (slope of the intensity $<1.5$ gray level/pixel). The particle peaks in this region were very tall and a standard low pass filter that gives all pixels some weight would not have completely removed the peaks. Close to the fish surface, where glare was greater and increased rapidly, a custom filter that forces monotonic growth in pixel intensity was applied (Fig. 5.3). When a spike (i.e. often a particle) in the slope of the plateau was encountered, the filter removed all points greater than and previous to the uphill-side minimum of the spike. Spikes up to a given width based on particle size were removed. The remaining pixel column following the application of the two filters represented the glare. This was subtracted from the original pixel column yielding a particle field with near zero background intensity. The algorithm preserves the distribution of each particle's intensity minus the level of glare so that accurate centroids can be determined even after glare is removed. To avoid possible errors due to the de-glaring process the original images can still be used to find centroids once the particles are located in the deglared images. Due to negligible errors this was found to be unnecessary. 


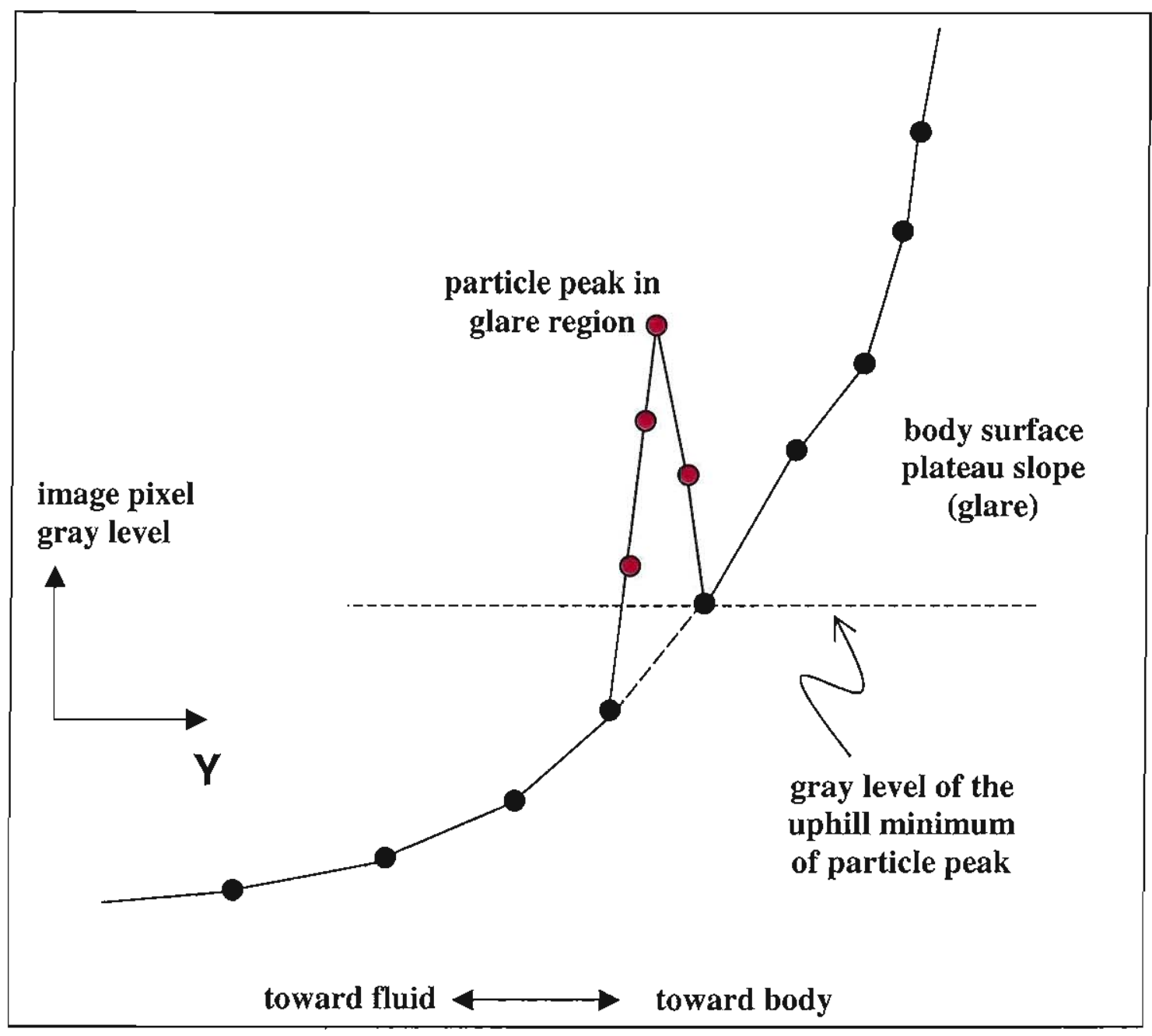

Fig. 5.3 Illustration of the 'forced-monotonic' glare filter used on the slope of the body surface plateau. The slope is due to glare. The points in red are removed because they do not represent monotonic progress of the glare. Once the peaks are removed, the glare is substracted from the original pixel column leaving only the particle peaks. 


\subsubsection{Particle centroiding}

The de-surfaced and de-glared image pairs were then subjected to simple image thresholding to remove noise from the de-glaring process. The remaining blobs of intensity were assumed to be particles and the centroid of each particle was determined using the center of intensities in the rectangular region circumscribing the particle. Since the glare (i.e. background) was removed in the de-glaring step, the non-particle pixels in the rectangle do not affect the calculation. This method allows for sub-pixel accuracy and was used instead of a Gaussian fit to reduce processing time. Most images had hundreds to thousands of particles. The particle centroids, $P(X, Y)$, were determined for both images of each image pair to be processed.

\subsubsection{Particle tracking by track convergence velocimetry}

The human eye is able to track particles in successive images of high shear flow without knowing anything in advance about the flow. This is apparently due to the fact that small clusters of particles within the shear layer can be recognized as moving in concert. This is the principle of conventional DPIV, but the human eye is not restricted to fixed sub-windows and performs better. Even two particles can be enough of a pattern for the brain to follow from image to image. The similarity in the motion of the two particles distinguishes their tracks from other possible tracks. The particle tracking code developed in this investigation takes advantage of this observation and will be referred to as particle track convergence velocimetry, PTCV.

Given a randomly distributed particle field, if one plots direction angle vs. distance of all of the possible tracks of all the particles in a pair of sequential images, clusters of points will occur for groups of two or more particles that travel on similar paths. Clusters reflecting a density some degree higher than what would be expected for two random particle fields can be located on the angle vs. direction plot. The 
corresponding tracks can then be selected and further filtered by limiting each particle to only one track. This algorithm works exceptionally well for the boundary layer, and shear layers in general, since it does not even require that the particle groups be made up of near neighbors. Since in the small field of view used to image flow in the boundary layer tangential velocity, $u$, is primarily a function of the distance from the body surface, $y$, the successive 'velocity layers' of the shear layer represent large groups of particles moving with similar velocity. The algorithm resulted in particle tracking success in the boundary layer that actually exceeded the manual particle tracking of the preliminary investigation. The following paragraphs describe the algorithm in detail.

A simplified, computer-generated image pair will be used here to illustrate the particle tracking algorithm (Fig. 5.4). Image 1 includes particles A-F. Image 2 includes particles 1-5. Particles D, E, and F have moved to the positions labeled as particles 3, 4, and 5, respectively. Particles $A$ and $B$ have moved to 1 and 2 . A small amount of random error has been added to simulate real experimental data. Particle $\mathrm{C}$ moves out of the field of view. For simplicity, in this example the body surface is a stationary, straight line.

Once particle centroids are determined, the set of all possible tracks between particles $\mathrm{A}-\mathrm{F}$ in image 1 and particles $1-5$ in image 2 are determined. Fig. 5.5 is a plot of the particles $A-F$ and $1-5$ from images 1 and 2 connected by the set of possible tracks A1-F5 (designated by start and end location, i.e., track AI refers to a track from particle A to particle 1). Fig. 5.6 illustrates a 'track plot' of the possible tracks Al-F5, and a close-up of one area of interest. The $x$-axis of the track plot corresponds to track length and the $y$-axis of the track plot corresponds to track angle. In this way, each data point on the track plot represents an individual track A1 - F5. A track plot typically shows considerable scatter since most of the possible tracks A1-F5 are erroneous and the track 

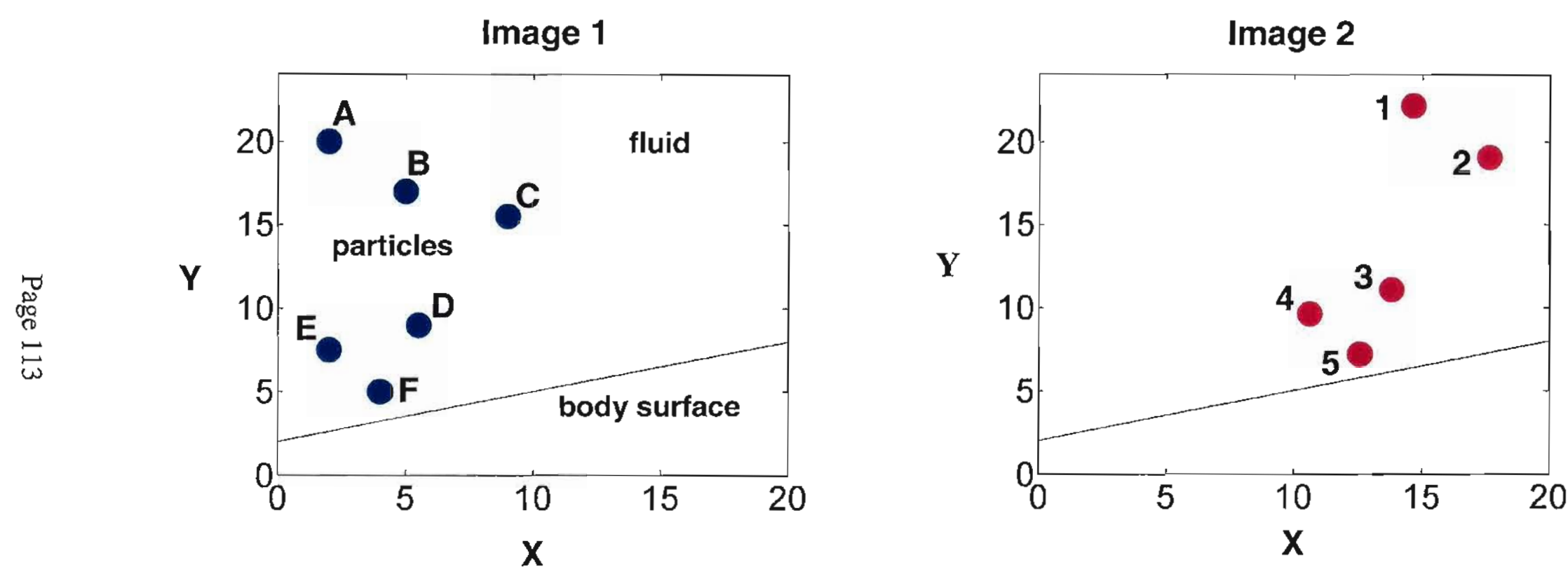

Fig 5.4 Computer generated image pair used to illustrate the particle tracking algorithm used in this investigation. The units of $X$ and $Y$ are unimportant, but could represent pixels or millimeters. Particle images in image 1 are designated by letters $A-F$, and in image 2, by numbers $1-5$. Particle $C$ has left the field of view in image 2 . 


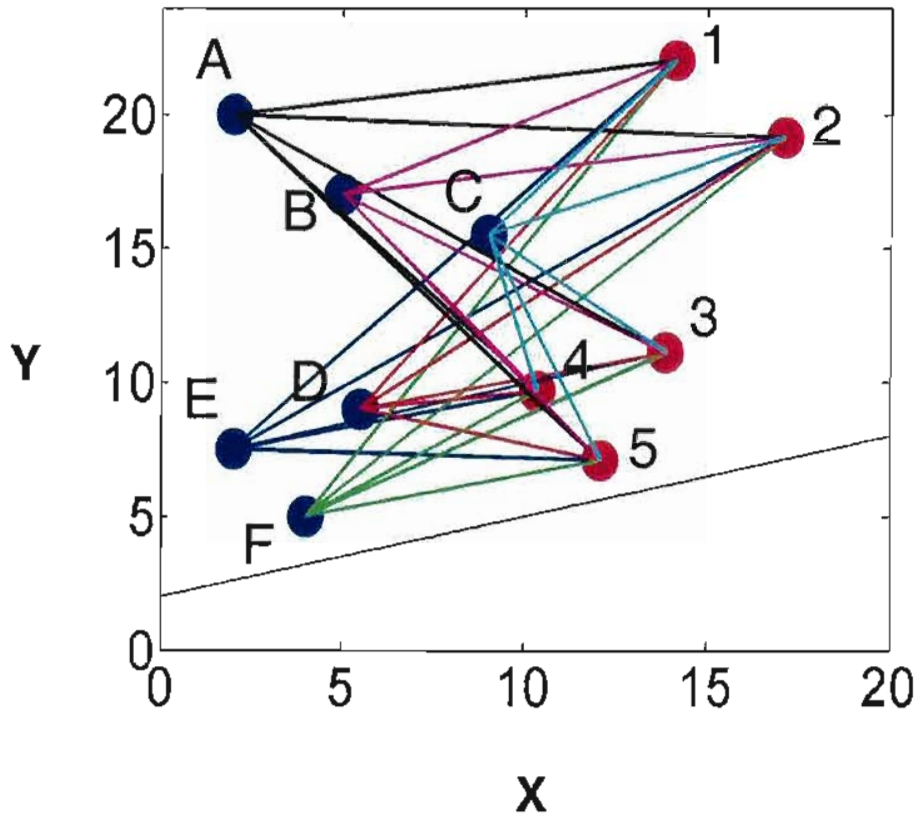

Fig. 5.5 The plot of all potential tracks between particle images $A-F$ and $1-5$. 


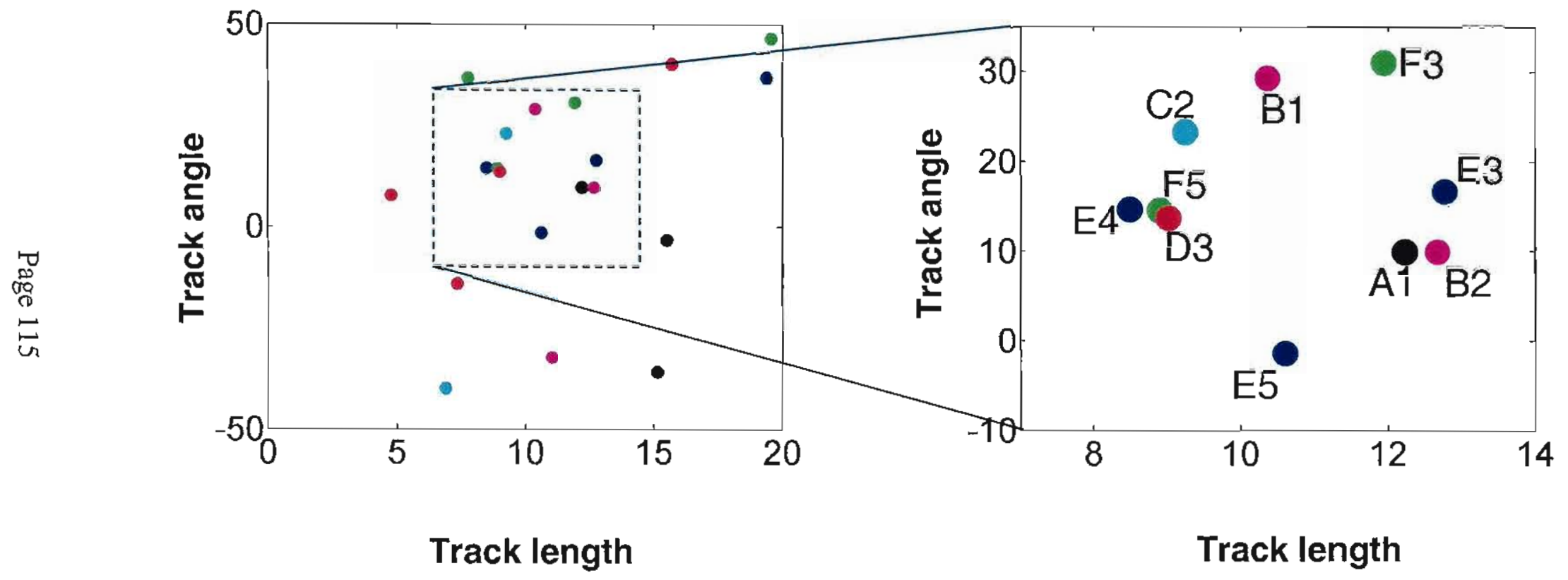

Fig. 5.6 The track plot for the particle tracks of Fig. 5.5. Showing a close up of the region showing the highest densities of tracks. 
angles and lengths land randomly on the plot. However, the tracks of groups of particles traveling at nearly the same velocity, that is, having nearly the same track angle and length (as would be expected in a continuous flow field, particularly at distances equidistant from a surface), land near each other on the track plot. That is, clusters of tracks appear on the track plot for tracks representing the actual particle tracks. The close-up includes an area of the track plot that exhibits a higher density of potential tracks Al-F5.

In order to locate tracks in high density regions on the track plot, and thereby determine the actual tracks, each potential track A1-F5 is scored based on the nearness of other potential tracks A1-F5 surrounding it. Rather than calculate all the distances between all points on the track plot, the track plot is transformed into an image so that more efficient image processing schemes can be used to calculate local track densities on the track plot. The track plot is scaled and digitized, for example, into a 1,000 by 1,000 'track matrix', or 'track image'. The size of the track matrix can be set to other sizes depending on a user's desired resolution in the analog to digital transformation of the track plot. Fig. 5.7 illustrates a section of the track matrix corresponding to the close-up section of the track plot from Fig. 5.6. Each track matrix entry is given a value equal to the number of potential tracks A1-F5 that fall within the location on the track plot corresponding to the matrix entry. Conceptually, if one were to divide the track plot into a grid, the value of each track matrix entry would be equal to the number of potential tracks A1-F5 falling within a particular grid square to which the matrix entry corresponds.

The density score for each potential track is determined by a kernel multiplication method (Fig. 5.8). For example, Fig. 5.8B shows the region of the track matrix surrounding track F5. The colored grid squares in the matrix have values of 1 , corresponding to potential tracks $\mathrm{C} 2, \mathrm{D} 3, \mathrm{E} 4$, and F5 seen in the close-up of the track plot. Fig. 5.8A is an illustrative kernel generated by the particle tracking code. The 


\begin{tabular}{|c|c|c|c|c|c|c|c|c|c|c|c|c|c|c|c|c|c|c|c|c|c|c|c|}
\hline 0 & 0 & 0 & 0 & 0 & 0 & 0 & 0 & 0 & 0 & 0 & 0 & 0 & 0 & 0 & 0 & 0 & 0 & 0 & 0 & 0 & 0 & 0 & 0 \\
\hline 0 & 0 & 0 & 0 & 0 & 0 & 0 & 0 & 0 & 0 & 0 & 0 & 0 & 0 & 0 & 0 & 0 & 0 & 0 & 0 & 0 & 0 & 0 & 0 \\
\hline 0 & 0 & 0 & 0 & 0 & 0 & 0 & 0 & 0 & 0 & 0 & 0 & 0 & 0 & 0 & 0 & 1 & 0 & 0 & 0 & 0 & 0 & 0 & 0 \\
\hline 0 & 0 & 0 & 0 & 0 & 0 & 0 & 0 & 0 & 0 & 0 & 0 & 1 & 0 & 0 & 0 & 0 & 0 & 0 & 0 & 0 & 0 & 0 & 0 \\
\hline 0 & 0 & 0 & 0 & 0 & 0 & 0 & 1 & 0 & 0 & 0 & 0 & 0 & 0 & 0 & 0 & 0 & 0 & 0 & 0 & 0 & 0 & 0 & 0 \\
\hline 0 & 0 & 0 & 0 & 0 & 0 & 0 & 0 & 0 & 0 & 0 & 0 & 0 & 0 & 0 & 0 & 0 & 0 & 0 & 0 & 0 & 0 & 0 & 0 \\
\hline 0 & 0 & 0 & 0 & 0 & 0 & 0 & 0 & 0 & 0 & 0 & 0 & 0 & 0 & 0 & 0 & 0 & 0 & 0 & 0 & 0 & 0 & 0 & 0 \\
\hline 0 & 0 & 0 & t & 0 & 1 & 0 & 0 & 0 & 0 & 0 & 0 & 0 & 0 & 0 & 0 & 0 & 0 & 0 & 0 & & 0 & 0 & 0 \\
\hline 0 & 0 & 0 & 0 & 0 & 0 & 1 & 0 & 0 & 0 & 0 & 0 & 0 & 0 & 0 & 0 & 0 & 0 & 0 & 0 & 0 & 0 & 0 & 0 \\
\hline 0 & 0 & 0 & 0 & 0 & 0 & 0 & 0 & 0 & 0 & 0 & 0 & 0 & 0 & 0 & 0 & 0 & 0 & 0 & 0 & 0 & 0 & 0 & 0 \\
\hline 0 & 0 & 0 & 0 & 0 & 0 & 0 & 0 & 0 & 0 & 0 & 0 & 0 & 0 & 0 & 0 & 0 & 0 & 0 & 0 & 0 & 0 & 0 & 0 \\
\hline 0 & 0 & 0 & 0 & 0 & 0 & 0 & 0 & 0 & 0 & 0 & 0 & 0 & 0 & 0 & 0 & 0 & 1 & 0 & 1 & 0 & 0 & 0 & 0 \\
\hline 0 & 0 & 0 & 0 & 0 & 0 & 0 & 0 & 0 & 0 & 0 & 0 & 0 & 0 & 0 & 0 & 0 & 0 & 0 & 0 & 0 & 0 & 0 & 0 \\
\hline 0 & 0 & 0 & 0 & 0 & 0 & 0 & 0 & 0 & 0 & 0 & 0 & 0 & 0 & 0 & 0 & 0 & 0 & 0 & 0 & 0 & 0 & 0 & 0 \\
\hline 0 & 0 & 0 & 0 & 0 & 0 & 0 & 0 & 0 & 0 & 0 & 0 & 0 & 0 & 0 & 0 & 0 & 0 & 0 & 0 & 0 & 0 & 0 & 0 \\
\hline 0 & 0 & 0 & $a$ & 0 & 0 & 0 & 0 & 0 & 0 & 0 & 0 & 0 & 0 & 0 & 0 & 0 & 0 & 0 & 0 & 0 & 0 & 0 & 0 \\
\hline 0 & 0 & 0 & 0 & 0 & 0 & 0 & 0 & 0 & 0 & 0 & 0 & 0 & 0 & 0 & 0 & 0 & 0 & 0 & 0 & 0 & 0 & 0 & 0 \\
\hline 0 & 0 & 0 & 0 & 0 & 0 & 0 & 0 & 0 & 0 & 0 & 0 & 0 & & 0 & 0 & 0 & 0 & 0 & 0 & 0 & 0 & 0 & 0 \\
\hline 0 & 0 & 0 & 0 & 0 & 0 & 0 & 0 & 0 & 0 & 0 & 0 & 0 & 0 & 0 & 0 & 0 & 0 & 0 & 0 & 0 & 0 & 0 & 0 \\
\hline 0 & 0 & 0 & 0 & 0 & 0 & 0 & 0 & 0 & 0 & 0 & 0 & 0 & 0 & 0 & 0 & 0 & 0 & 0 & 0 & 0 & 0 & 0 & 0 \\
\hline 0 & 0 & 0 & 0 & 0 & 0 & 0 & 0 & 0 & 0 & 0 & 0 & 0 & 0 & 0 & 0 & 0 & 0 & 0 & 0 & 0 & 0 & 0 & 0 \\
\hline 0 & 0 & 0 & 0 & 0 & 0 & 0 & 0 & 0 & 0 & 0 & 0 & 0 & 0 & 0 & 0 & 0 & 0 & 0 & 0 & 0 & 0 & 0 & 0 \\
\hline
\end{tabular}

Track Length

Fig. 5.7 Transformation of the track plot of Fig. 5.6 to an 'image' to allow higher efficiency density calculations. A value of one is added to each matrix entry for each particle track landing in the entry square. The matrix is not the entire track plot. Instead it represents the close-up in Fig. 5.6. The gray region is used in Fig. 5.8. 


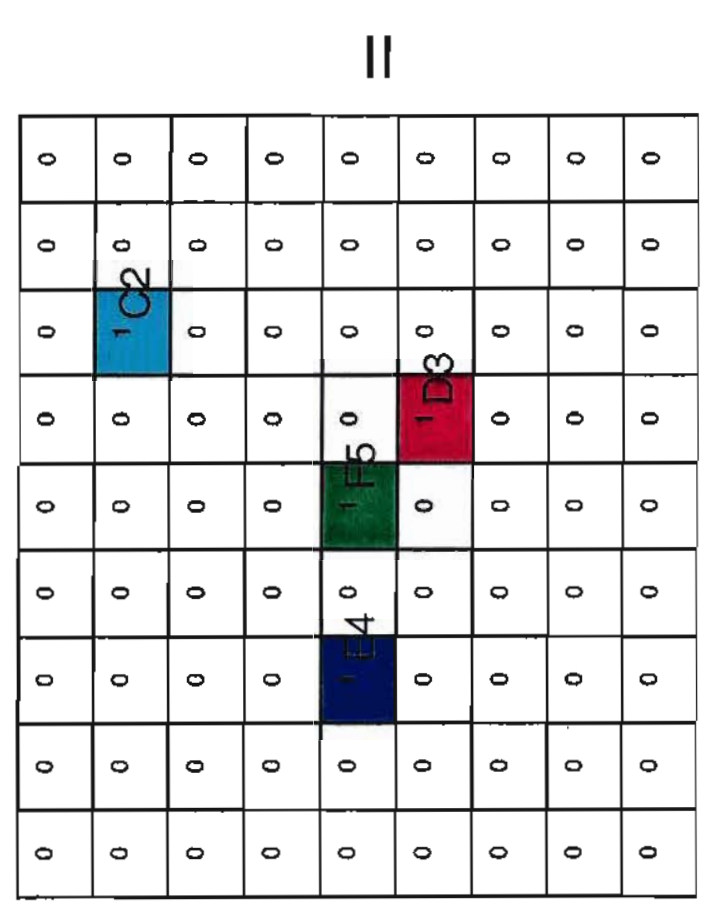

n

\begin{tabular}{|c|c|c|c|c|c|c|c|c|}
\hline 8 & $\stackrel{8}{8}$ & 8 & : & ? & : & 8 & O & $\stackrel{\circ}{\circ}$ \\
\hline 8 & $\stackrel{8}{\circ}$ & m & ? & g. & \% & $\stackrel{0}{\circ}$ & 8 & 8 \\
\hline 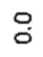 & $\ddot{m}$ & İ & ¿̊. & $\stackrel{\text { L }}{0}$ & $\stackrel{\square}{0}$ & ¿̊ & $\stackrel{m}{0}$ & $\stackrel{\circ}{\circ}$ \\
\hline 잉 & : & वे & ó & $\stackrel{\circ}{\div}$ & $\hat{o}$ & ¿̊ & $\stackrel{\infty}{0}$ & $\stackrel{\circ}{\circ}$ \\
\hline m & $\ddot{0}$ & 88 & $\stackrel{\circ}{-}$ & $\stackrel{\circ}{-}$ & $\stackrel{0}{-}$ & ஜூ & $\ddot{0}$ & \% \\
\hline$\stackrel{\circ}{0}$ & m & $\stackrel{\square}{0}$ & jo & $\stackrel{\circ}{\div}$ & $\hat{o}$ & : & m & 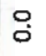 \\
\hline$\stackrel{\circ}{\circ}$ & $\ddot{0}$ & $\stackrel{\Delta}{0}$ & ¿艹 & $\stackrel{0}{0}$ & ¿̊ & : & $\stackrel{\infty}{0}$ & $\stackrel{8}{\circ}$ \\
\hline$\stackrel{8}{0}$ & $\stackrel{\circ}{\circ}$ & ? & $\stackrel{\text { g }}{0}$ & $\stackrel{m}{0}$ & $\stackrel{m}{0}$ & $\stackrel{m}{0}$ & 8 & 욤 \\
\hline : & $\stackrel{\circ}{\circ}$ & 응 & 号 & $\approx$ & 웅 & $\stackrel{\circ}{0}$ & $\stackrel{\circ}{\circ}$ & $\stackrel{g}{0}$ \\
\hline
\end{tabular}

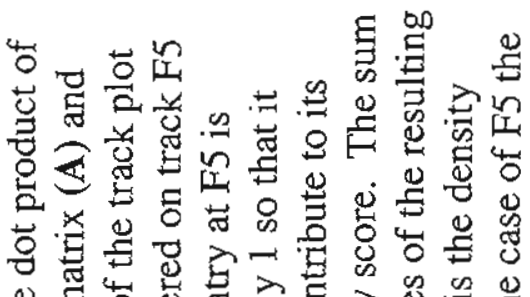

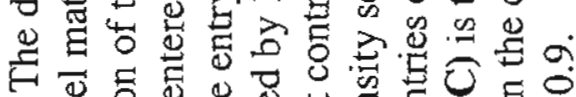

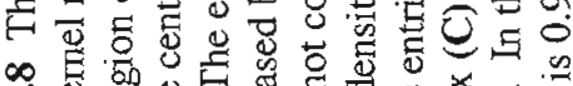

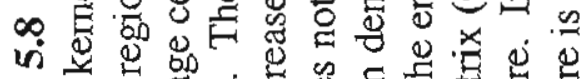

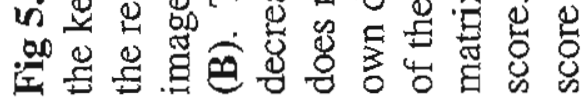

\begin{tabular}{|c|c|c|c|c|c|c|c|c|}
\hline$\stackrel{\circ}{\circ}$ & $\ddot{0}$ & $\ddot{\circ}$ & $\stackrel{\circ}{\circ}$ & $\stackrel{\circ}{0}$ & 8 & $\ddot{g}$ & 임 & 8 \\
\hline$\stackrel{\circ}{\circ}$ & $\stackrel{\circ}{\circ}$ & g. & $\stackrel{g}{0}$ & $\stackrel{8}{0}$ & \& & $\stackrel{g}{0}$ & 8 & 웅 \\
\hline$\stackrel{g}{\mathrm{~g}}$ & 5 & $\stackrel{8}{0}$ & $\stackrel{8}{0}$ & $\stackrel{8}{0}$ & $\stackrel{\circ}{0}$ & $\stackrel{\circ}{\circ}$ & 욤 & $\stackrel{\circ}{0}$ \\
\hline 응 & $:$ & $\stackrel{\circ}{\circ}$ & $:$ & $\stackrel{\circ}{0}$ & $\stackrel{\circ}{0}$ & $\stackrel{\circ}{\circ}$ & 8 & $\stackrel{8}{\circ}$ \\
\hline$\stackrel{\circ}{0}$ & $:$ & 용 & $\stackrel{\circ}{\circ}$ & $\stackrel{\circ}{0}$ & $\ddot{0}$ & : & $\ddot{\circ}$ & $: 8$ \\
\hline$\stackrel{8}{8}$ & $\stackrel{8}{\circ}$ & 옹 & 8 & 8 & 영 & 8 & 8 & 8 \\
\hline 8 & : & 8 & : & 8 & $\stackrel{8}{\circ}$ & $\stackrel{8}{8}$ & $\because$ & : \\
\hline$\stackrel{8}{\circ}$ & :영 & 염 & $\ddot{g}$ & 8 & 8 & 8 & $\stackrel{8}{\circ}$ & 잉 \\
\hline$\stackrel{8}{0}$ & 号 & $\stackrel{g}{0}$ & $\stackrel{\circ}{\circ}$ & $\stackrel{\circ}{0}$ & 응 & $\stackrel{\circ}{\circ}$ & $\stackrel{\circ}{\circ}$ & $\stackrel{\circ}{0}$ \\
\hline
\end{tabular}

Page 118 
kemel is a small matrix with dimensions approximately equal to the average distance between potential tracks A1-F5 on the digitized track plot. Potential tracks A1-F5 that fall within a circle with a diameter on the order of such a distance are less likely to be random scatter and are considered valuable in determining actual tracks. The entries in the kernel are equal to $1 / R_{i j}$, where $R_{i j}$ for any entry (row $i$, column $j$ ) is the distance from the center entry of the kernel to each entry (i.e. $\sqrt{i^{2}+j^{2}}$ where $i$ and $j=0$ at the center of the matrix). In addition, all the entries outside a circle inscribed by the outline of the kernel are set to zero. Basically, the kernel has a circular pattern of entries in which the values of entries increase from zero to very large as you move from the edge of the kernel toward the center. The center entry of the kernel is assigned a $1 / R$ value of 1 (i.e. $R=1$ ) to prevent a discontinuity. A value of 1 means that tracks that fall within the same track matrix entry are considered to be a distance of one matrix entry away. A Monte Carlo simulation suggests that the expected average distance between such tracks is 0.522 , corresponding to $1 / R=1.92$. The center value of $1 / R=1$ was used, nevertheless, to prevent over-weighting of errant tracks due to random occurrences of errant tracks landing in the same entry of the track matrix. The value 1.92 might be used in the case of low particle densities.

To determine the density score of a particular track, the kernel is overlaid onto the plot matrix, centering the kernel of the matrix entry of the track. Each potential track A1F5 is assigned a density score equal to the sum of the products of the overlapping kernel and track matrix entries (i.e. the dot product, Fig 5.8). The value of the track matrix entry over which the kernel is centered is reduced by 1 so that a particle track does not contribute to its own score. This is an additional safeguard against awarding high density scores to tracks when two errant tracks randomly land near each other on the track plot. For low particle densities this restriction could be relaxed. Based on the above process, potential tracks A1-F5 that have several other potential tracks A1-F5 located nearby are assigned high density scores, whereas much lower scores are assigned to isolated potential tracks (e.g., E5, Fig. 5.6). Fig. 5.8 is an illustration of the kernel multiplication. 
In the overlay, the kernel has been centered on potential track F5. Based on such an overlay, F5 would be granted a density score of 0.9 . The score is the sum of contributions provided by $\mathrm{C} 2(0.1), \mathrm{D} 3(0.5)$, and E4 (0.3). This conversion of the potential track scoring problem into one of image processing and matrix manipulation (i.e. utilizing the track matrix and kernel) turns out to be a remarkably efficient means of reaching a density score for each potential track. Processor time was measured to be proportional to the average number of particles, $n$, present in the analyzed images for up to $n=500$ particles. For comparison, code was written that calculated track density score for each potential track by sorting actual distances between each and every potential track to locate nearest neighbors on the analog track plot. That code used processor time proportional to $n^{4}$. At $n=100$ particles, the kemel based code took just 85 ms to rank all 10,000 potential tracks. This was 1200 times faster than the nearest neighbor calculating code.

After the potential tracks are scored, they are ranked, or sorted, by their respective density scores (Fig. 5.9). All potential tracks with a density score less than $10 \%$ of the average density score were immediately rejected. This threshold parameter can be changed by the user. The value $10 \%$ was found to work well in the current investigation. In an iterative fashion, the highest ranked potential track in the list of remaining potential tracks is assumed to be an actual particle track. All remaining potential tracks A1-F5 containing the start or end particle images of the chosen track are then eliminated from the list of potential tracks (e.g., if the top ranking potential track is track B2, all other potential tracks including particles $B$ or 2 are removed from contention). The algorithm repeats this process on the next highest ranking potential track. The remaining viable potential tracks may be re-ranked prior to choosing the next actual track to limit the effect of errant tracks on density scoring, but this increases processing time and was not used here. Fig. 5.10 shows the end result velocity field for the example used in the discussion above. 


\section{Track elimination process by ranking}

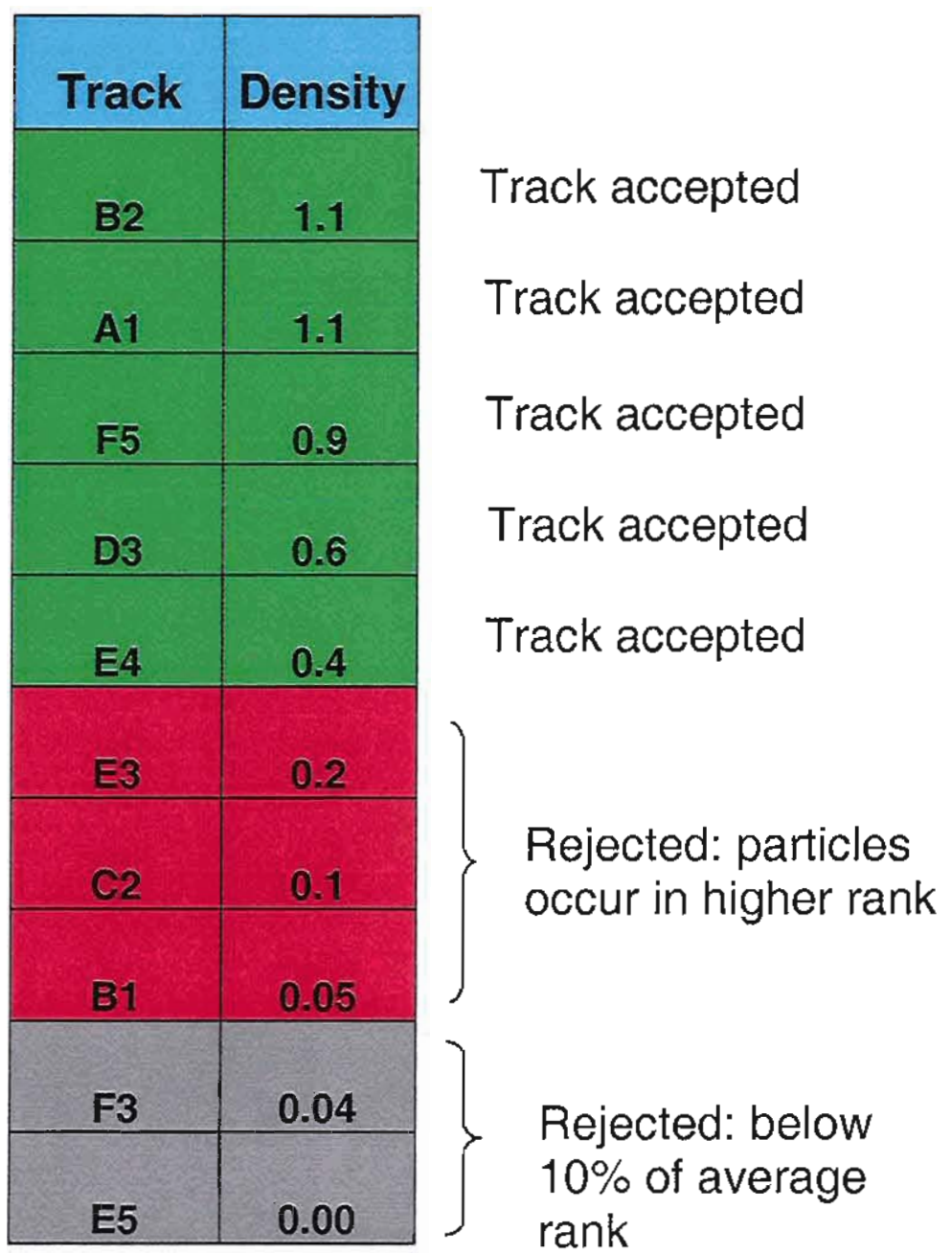

Fig. 5.9 Determination of actual tracks by density score. Selection begins at the top. The highest ranking track is assumed to be an actual track. Then all tracks including the particle images of the chosen track are removed from contention 


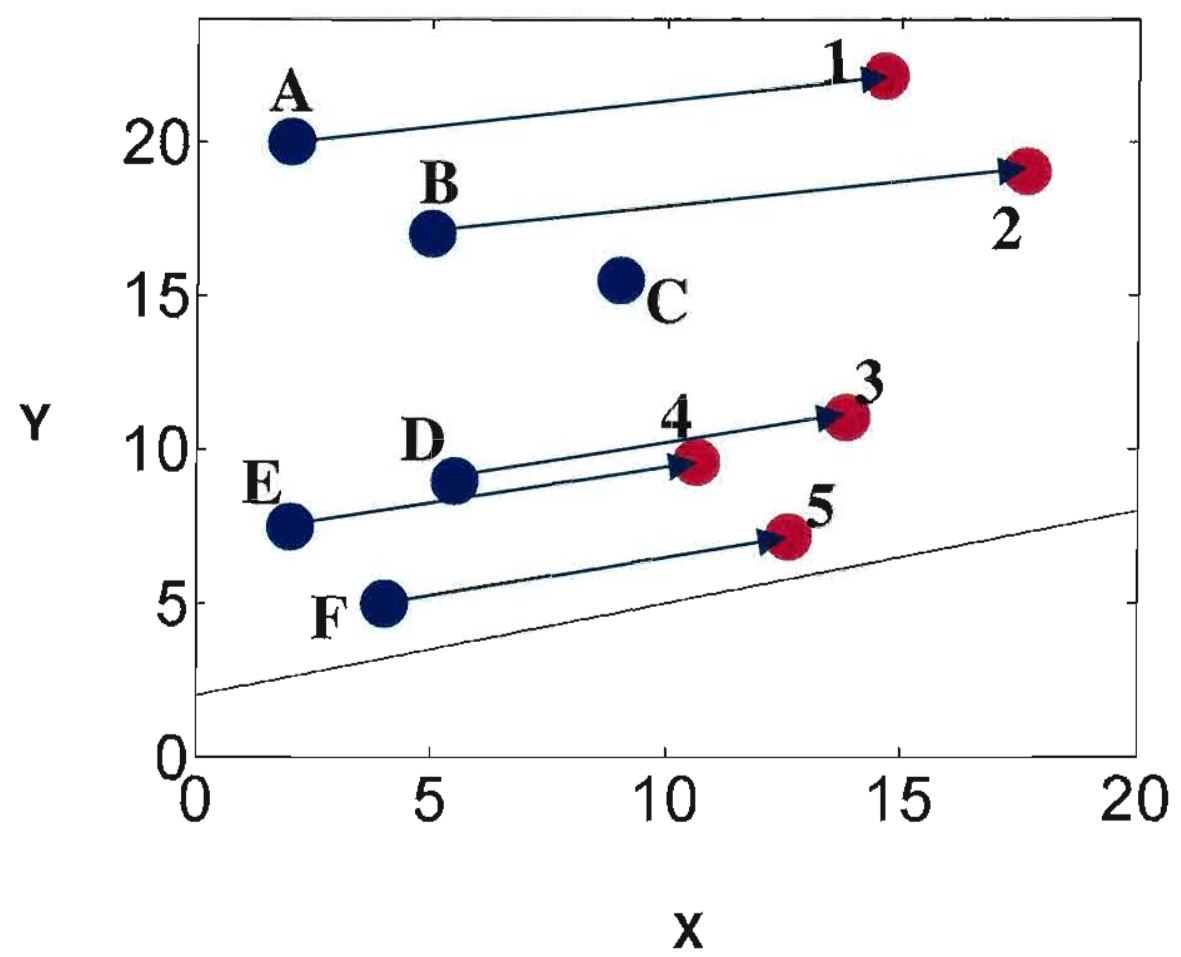

Fig. 5.10 The results of the particle tracking calculation by the particle track convergence algorithm. 
Figs. 5.11-16 show three examples of boundary layer profiles from the flat plate and live fish experiments using the particle tracking code described above. The first example is the flow $10 \mathrm{~cm}$ upstream from the flat plate with a blank inlet barrier and $U=$ $33.0 \mathrm{~cm} / \mathrm{s}$ (Figs. $5.11-12$ ). Note the highly uniform $u$-profile. The second example is from the same trial, but at $x=21.3 \mathrm{~cm}$ on the flat plate (Figs. 5.13-14). Blasius fits the boundary layer data with an $R^{2}$ of $0.99 . C f_{x}=0.0030$ and $R e_{x}=6.7 \times 10^{4}$. Particles are tracked to within 13 pixels (173 um) of the wall and $95 \%$ confidence limits on the slope of the profile at the wall are $0.6 \%$. Standard deviation in the slope is $2.6 \%$, and the expected error due to the assumption that the slope near the wall is the same as the slope at the wall is $2.6 \%$ (see section 6.2.6). This translates into $95 \%$ confidence limits of $3.5 \%$ in the determination of $C f_{x}$, and a maximum expected error of $8.1 \%$. The profile is clearly not fit well by the law of the wall (Fig. 5.13D) except in the linear region close to the wall. The law of the wall fit shown in Fig. 5.13D was constrained to fit in the linear region and resulted in an $R^{2}$ value less than 0.01. If not constrained in this way $R^{2}=0.40$ and the linear region is poorly fit, which result in a significantly inaccurate value for the slope of the real profile at the wall.

The third example profile is from a bluefish swimming $24 \mathrm{~cm} / \mathrm{s}(0.55 \mathrm{~L} / \mathrm{s}), x=$ $32.3 \mathrm{~cm}$ (Figs. 5.15-16). Blasius fits the data with an $R^{2}$ of 0.98. $C f_{x}=0.0030$ and $R e_{x}=$ $7.6 \times 10^{4}$. As in the flat plate example, particles are tracked to within 13 pixels of the wall and the expected error due to the assumption that the slope near the wall is the same as the slope at the wall is $2.6 \%$. $95 \%$ confidence limits on the slope of the profile at the wall are $0.4 \%$ and standard deviation is $2.4 \%$. $95 \%$ confidence limits in the determination of $C f_{x}$, are $+/-3.1 \%$ and maximum expected error is $7.8 \%$. Fits to the law of the wall, constrained and unconstrained, resulted in $R^{2}$ values of less than 0.07 . Note that 344 particles were tracked in the boundary layer. Fig. 5.17A shows the failure of conventional DPIV to resolve the swimming fish boundary layer (Figs. 5.15, 5.17B). 

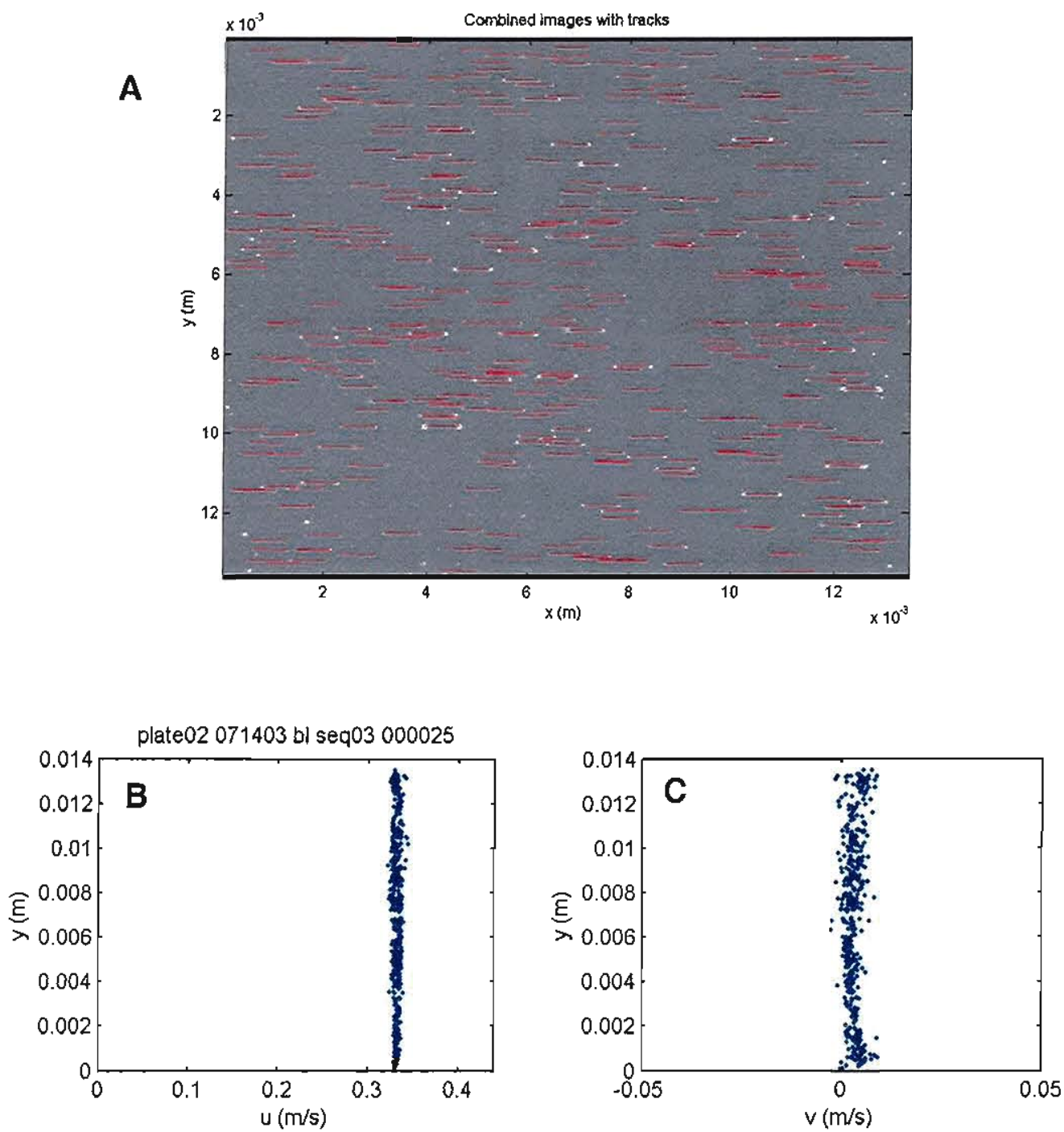

Fig. 5.11 Superimposed image pair (A) with particle tracks and velocity profiles $(B, C)$ determined by PTCV from flow $10 \mathrm{~cm}$ upstream of the flat plate taken with the boundary layer camera. Flow is from left to right. The inlet barrier type was blank, $U=33.0 \mathrm{~cm} / \mathrm{s}$. RMS $u / U=1.0-1.5 \%$. Note that the velocity scale for the $v$-profile is much smaller than that of the $u$-profile, therefore the relative error appears to be greater than it is. In reality, it is comparable to that seen in $u$. The slightly positive $v$-velocity is evidence of slight cross-flow toward the center of the flume. 


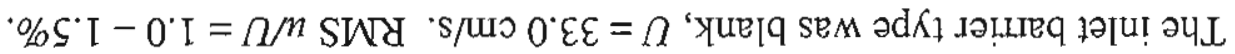

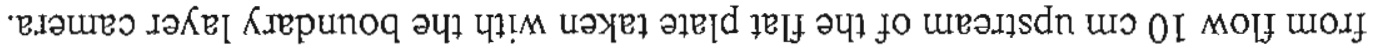

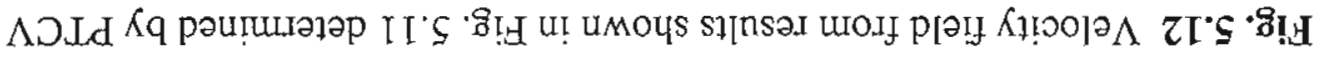

(w) $x$

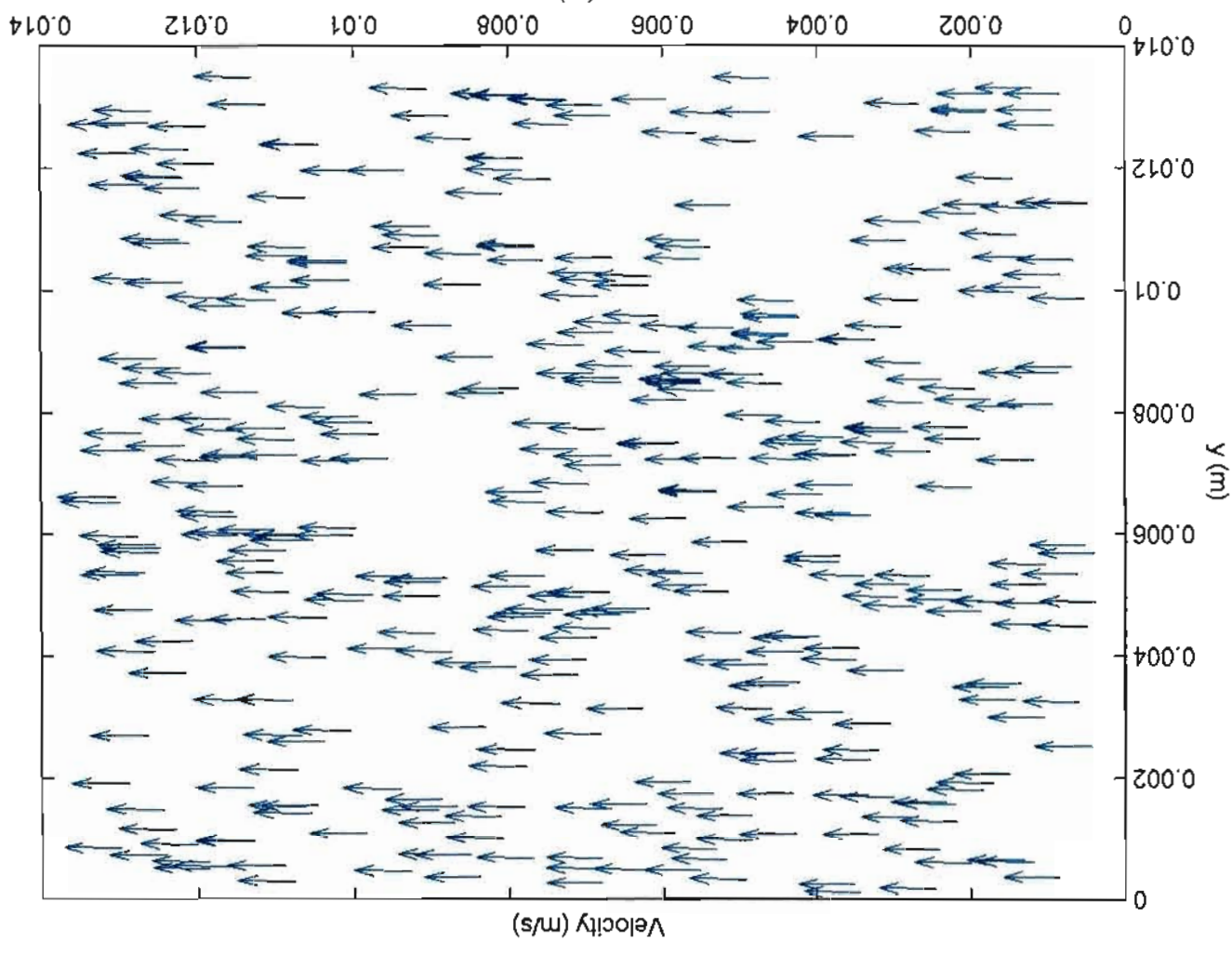



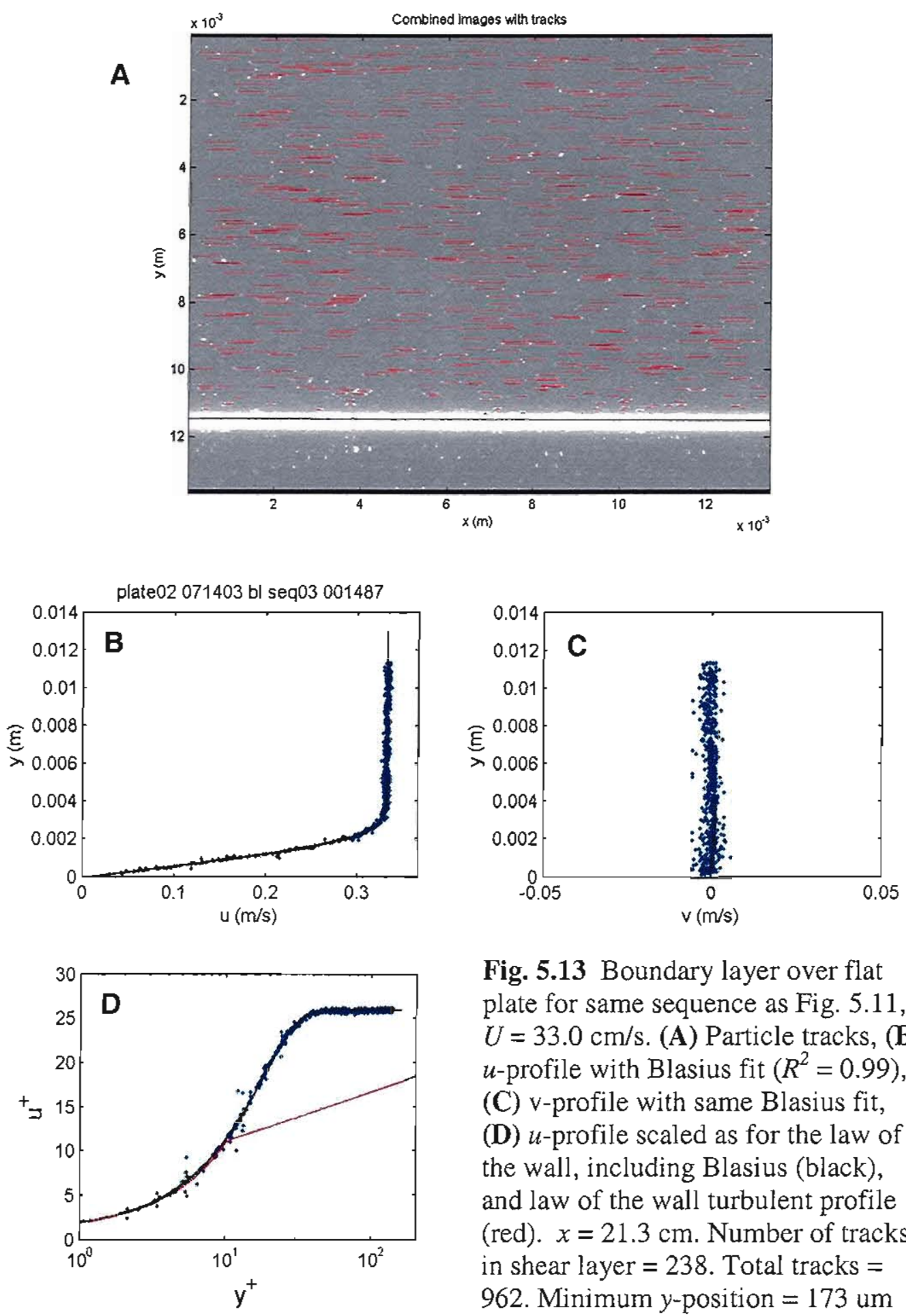

Fig. 5.13 Boundary layer over flat plate for same sequence as Fig. 5.11, $U=33.0 \mathrm{~cm} / \mathrm{s}$. (A) Particle tracks, (B) $u$-profile with Blasius fit $\left(R^{2}=0.99\right)$,

(C) v-profile with same Blasius fit, (D) $u$-profile scaled as for the law of the wall, including Blasius (black), and law of the wall turbulent profile (red). $x=21.3 \mathrm{~cm}$. Number of tracks in shear layer $=238$. Total tracks $=$ 962. Minimum $y$-position $=173 \mathrm{um}$ (13 pixels). $C_{f x}=0.0030+/-3.5 \%$ ( $95 \%$ confidence limits). 


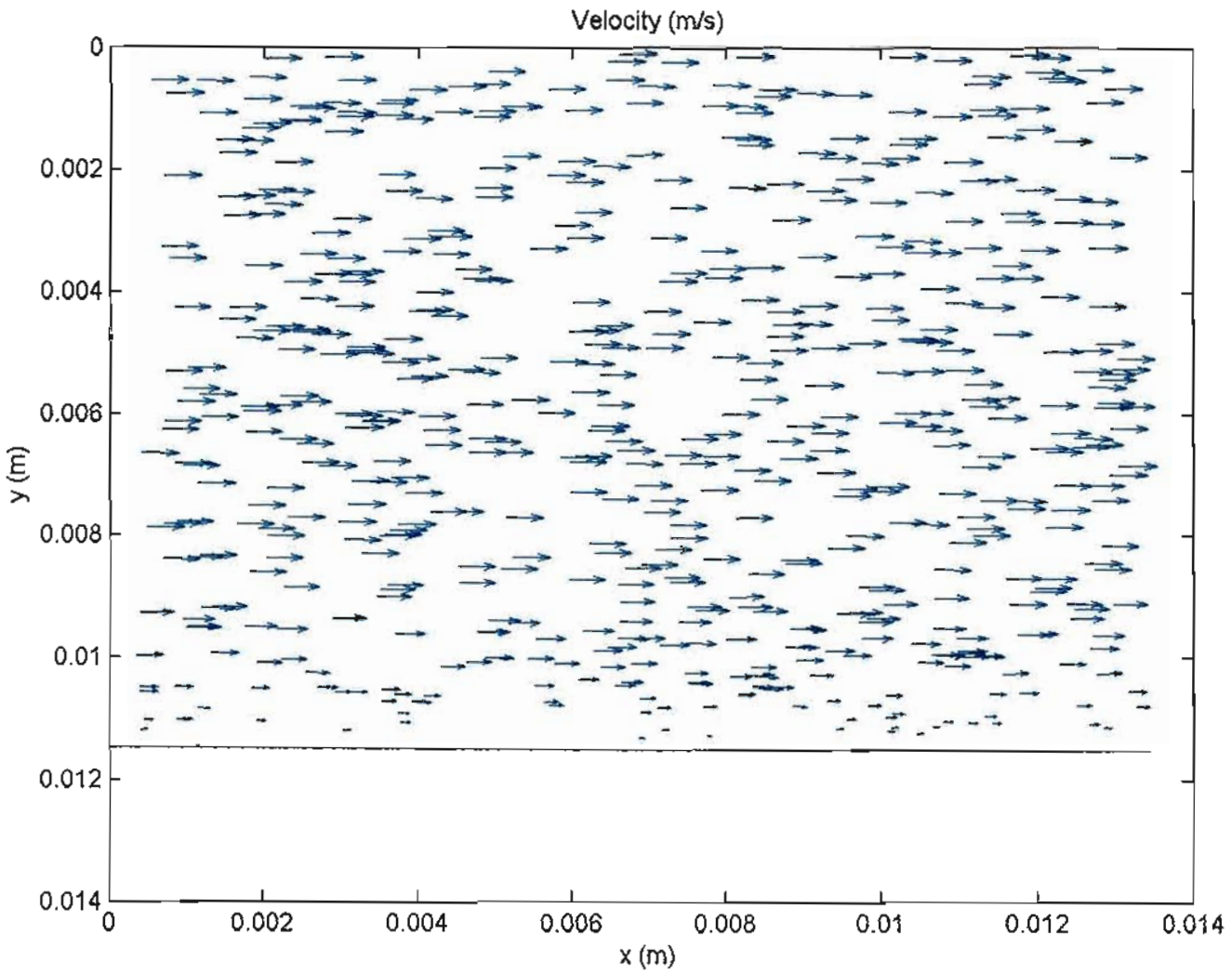

Fig. 5.14 Velocity field from results shown in Fig. 5.13 determined by PTCV from flow at $x=21.3 \mathrm{~cm} / \mathrm{s}$ over a flat plate taken with the boundary layer camera. The inlet barrier type was blank. $U=33.0 \mathrm{~cm} / \mathrm{s}$. 

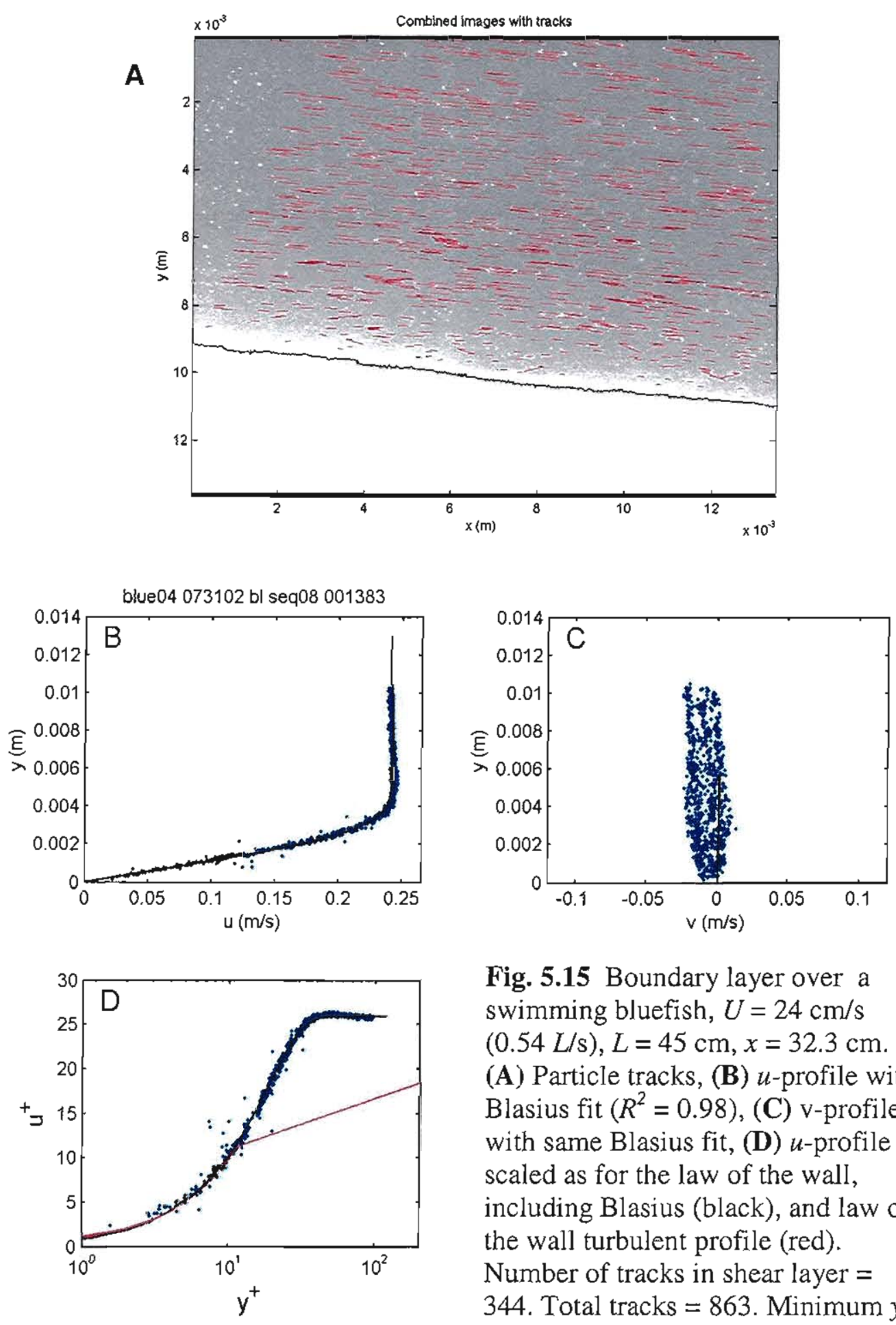

Fig. 5.15 Boundary layer over a swimming bluefish, $U=24 \mathrm{~cm} / \mathrm{s}$ ( $0.54 \mathrm{~L} / \mathrm{s}), L=45 \mathrm{~cm}, x=32.3 \mathrm{~cm}$. (A) Particle tracks, (B) $u$-profile with Blasius fit $\left(R^{2}=0.98\right)$, (C) v-profile with same Blasius fit, (D) $u$-profile scaled as for the law of the wall, including Blasius (black), and law of the wall turbulent profile (red). Number of tracks in shear layer $=$ 344. Total tracks $=863$. Minimum $y$ position $=170$ um (13 pixels). $C_{f x}=$ $0.0030+/-3.1 \%$ (95\% confidence limits). 


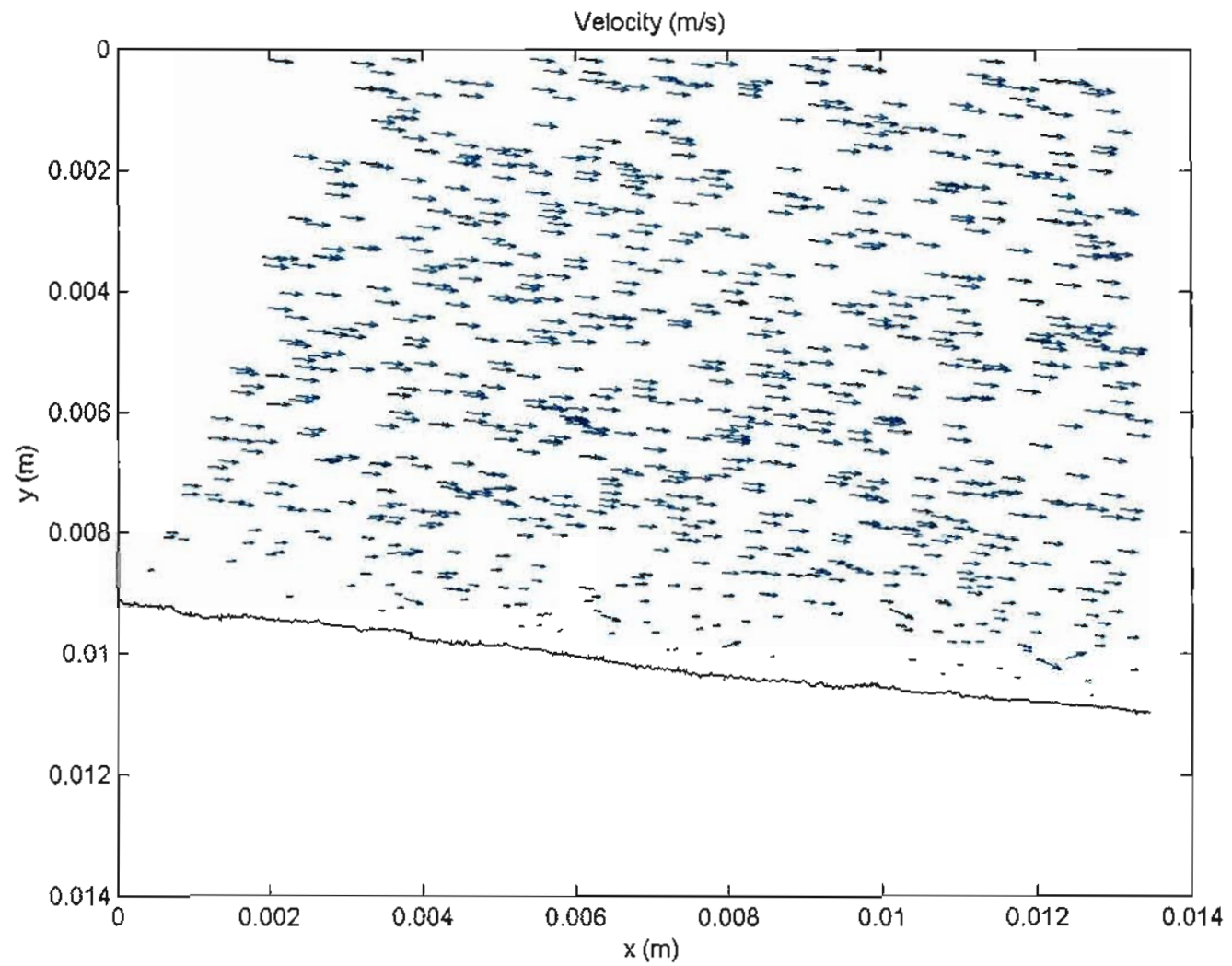

Fig. 5.16 Velocity field from results shown in Fig. 5.15 determined by PTCV from flow at $x=32.3 \mathrm{~cm}$ along a swimming bluefish $(L=45.0 \mathrm{~cm})$ taken with the boundary layer camera. $U=24 \mathrm{~cm} / \mathrm{s}$. The blank area in the upper left-hand comer is to the left of the approximate surface normal at the left-most edge of the image. Particles that do not allow for a measurement of normal distance to the surface are not usable in producing boundary layer profiles and are not tracked. 

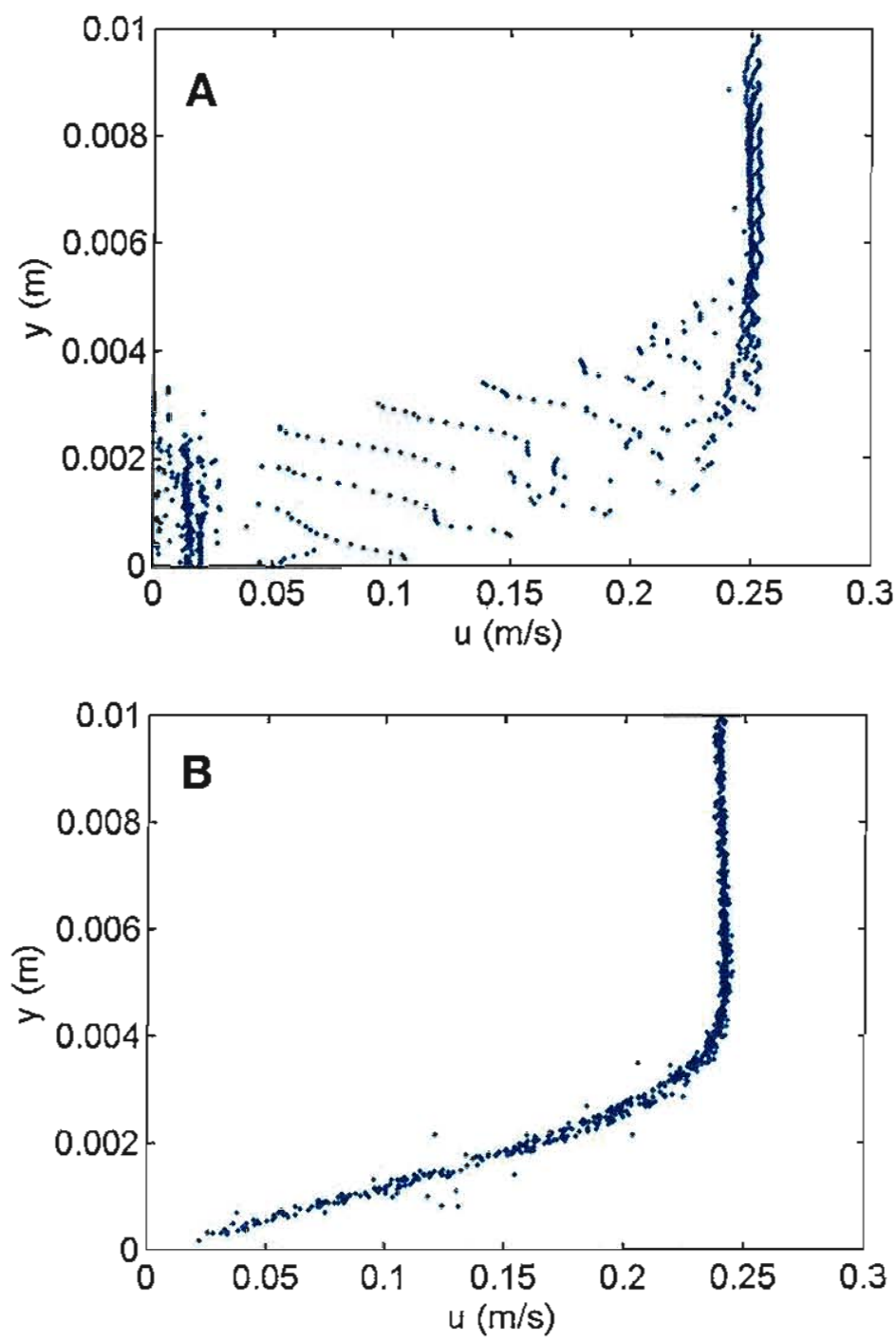

Fig. 5.17 Comparison of the results of (A) conventional DPIV and (B) the particle tracking code developed in this investigation to resolve the boundary layer of a swimming fish. The profile used is the bluefish boundary layer profile from Fig. 5.15. 
The code includes a feature to deal with highly curved surfaces. When this feature is turned on, track angle is calculated with respect to the surface. Since the motion of particles in the boundary layer near a surface is influenced by the surface this can improve track convergence. Particles some distance away from each other along the surface, but at a similar distance from the surface, may trace out similar tracks with respect to the surface. If track angle were not determined with respect to the curved surface, the tracks traced out by these particles with respect to the field of view of the image will be angularly dissimilar. If particle density is high enough, the step of determining the track angle with respect to the surface can be skipped to reduce processing time. Surface curvature in the small field of view of the boundary layer camera and good particle densities made this feature unnecessary in this investigation.

To reduce processing time and avoid errors inherent in mapping too many potential tracks on the track plot, the flow over the surface of the fish was cut into slices parallel to the surface. Recall that errors can occur when errant tracks randomly end up in the same track matrix entry. This is more likely when larger numbers of potential tracks are mapped to the track plot. The code can be used for any sub-window shapes and the shapes can be non-uniform throughout the image, but slices were chosen since the largest velocity gradient in the boundary layer varies in the $y$-direction. It is supposed that particles in the slices move with similar velocities. Nevertheless, as should be understood from the preceding discussion, the code does not require this to be the case. The code looks at particle groups, not the average motion of particles within the region analyzed. This is the main advantage of the algorithm. Slicing the flow parallel to the surface of the fish simply increases the likelihood of stronger convergence of tracks in the track plot in the case of boundary layer flow. Slices may, but need not overlap. Slice dimensions preferably are large enough to encompass greater than about 10 particles per slice, and slices including up to several hundred particles will improve performance. Preferably, the slices used to divide image 2 are thicker than those used to slice image 1. The increase in thickness should be the maximum distance a particle would be expected 
to travel perpendicular to the interface, $d y_{\max }$, with respect to the interface. This value is usually very small near interfaces. Therefore, one need not know very much about the flow at all in order to select an appropriate slice size, especially if the slice thickness in image 1 is at least $2-3$ times larger than the expected $d y_{\max }$. In this investigation, slices were further sectioned by cutting them at $1-2$ streamwise positions along the surface of the fish.

In theory, the particle tracking algorithm described above does not need any user input concerning the flow being analyzed. In practice, certain aspects of the flow are known, such as maximum expected velocities, especially within the boundary layer. Therefore, to save computing time, all possible tracks need not and were not calculated. Note, however, that this does not define the resolution of the boundary layer, as in the case of conventional DPTV. For example, even within the strips used in this investigation, separate groups of particles with unique track angles and distances will form separate clusters on the track plot that can be independently located. In fact, even within clusters of similar tracks, the track of each particle and therefore slight trends in velocity are preserved for the eventual plotting of the flow field and boundary layer profiles. This results in very high resolution of the flow. For example, for a laminar boundary layer over a flat plate, if a thick interrogation strip is used, the track plot exhibits an elongated cluster along track angle $=0$. This is because the particles throughout the strip travel roughly parallel to the flat plate (track angle $=0$ ), but particles travel longer distances the farther they are from the plate. All of these particle tracks are part of a cluster and can be determined to be actual tracks by the algorithm described here, even though they represent a steep velocity gradient. Turbulent flow is a problem for any pattern based tracking code, however the present code proved to be so robust for laminar and low-level turbulent flows that if good seeding particle density was present, the failure to converge indicated very turbulent flow. This was observed in the experimental controls (see Chapter 6). 
The method of calculating all possible tracks as a starting point for particle tracking was also employed by Kim and Chen (1992) and Wernet (1993). Kim and Chen (1992) go on to produce a different sort of track image, which they call a 'digital vectorgram', using Cartesian vector components rather than the polar coordinates used in this investigation. This technique reduces the number of calculations needed to produce the track plot. Nevertheless, Kim and Chen (1992) only use the technique to determine average velocities in square sub-windows, as in conventional DPIV. Rather than scoring each potential track, they determine the center of intensity on their digital vectorgram. The problem with this technique is that errant tracks contribute to the final velocity determined. Not surprisingly, they report that their code failed to resolve areas of steep velocity gradients. The information of the individual particle tracks is lost in the averaging. The choice of polar coordinates in the current investigation was to give the code the flexibility to deal with highly curved surfaces. As mentioned earlier, surface curvature was not a significant problem in this investigation, therefore updating the code to Cartesian coordinates would reduce processing time. Test code was found to calculate the track plot $10-20 \%$ faster using Cartesian components. However, the choice of coordinate system should not impact resolution. Wernet (1993) uses the technique of calculating potential tracks but does not use a track plot, track image or kernel multiplication method to score and select actual tracks. Instead, he uses a fuzzy logic processor to determine actual tracks.

\subsection{Boundary layer profile calculation}

Tangential and normal velocity profiles of the boundary layer $(u=u(y)$ and $v=$ $v(y))$ were determined from the results of the particle tracking in the same way as in the preliminary experiments except for two important differences. First, the normal velocity profile was determined using a vector dot product method as used for the tangential velocity profile in the preliminary experiments. Second, smoothing of the surface of the 
fish was performed independently for each particle track based on the distance of the track midpoint from the surface.

The equations for determining the tangential and normal velocities for each particle track of any image pair in the current investigation were,

$$
\begin{aligned}
& u=\left(V_{p}-V_{s}\right) \cdot t \\
& \mathrm{v}=\left(V_{p}-V_{s}\right) \cdot n
\end{aligned}
$$

where $t$ and $\boldsymbol{n}$ are unit vectors tangential and normal to the average surface of the fish in the processed image pair, respectively; $V_{p}$ is the determined velocity vector of any given tracked particle and $V_{s}$ is the velocity vector of the fish surface. The unit vectors, $t$ and $n$, were determined for each particle track at the point where a normal drawn from the midpoint of the track intersected the average surface. The unit tangent vector was constructed tangent to the average fish surface, in a streamwise sense, and the unit normal vector was constructed perpendicular to the average fish surface pointing outward toward the fluid. All vectors $t, n, V_{p}$ and $V_{s}$ were determined with respect to the camera pixel coordinates. Contrary to the conclusions of the preliminary experiments, the dot product method of determining the normal velocity profile (Eq. 5.2) was found to produce slightly less scatter in the profile than Eq. 3.1. The determination of an average surface does not consistently appear to affect the profile as was suggested in the preliminary work.

As mentioned above, the surface of the fish was smoothed independently for the treatment of each particle track. A shorter sample of the surface was taken for tracks that were closer to the surface. Thus the local surface geometry had more impact on the smooth curve used to calculate the velocity components and location of the particle track with respect to the surface of the fish. This made the regions of the boundary layer 
profiles close to the body surface more accurate. The preliminary experiments used a cubic fit of the entire surface in the field of view of the boundary layer camera. This can potentially lead to large errors in resolving the regions of the boundary layer nearest to the surface. The sample length, $\ell_{s}$, used was a value 1 to 3 times the distance of the track midpoint to the average surface as estimated by a cubic fit of the entire surface. Distances of track midpoints to the average surface of the fish were determined by the minimization of the distance routine described for the preliminary experiments. A sample with length, $\ell_{s}$, of the average surface, centered on the intersection of the normal to the surface drawn from the track midpoint, was fit with a cubic polynomial. The distance of the track midpoint to the surface was then refined, unit vectors were determined and the components of velocity, $u$ and $v$ were calculated using Eqs. 5.1 and 5.2 .

It should be mentioned here that normal velocity is somewhat problematic when dealing with a moving surface. For example, consider a surface moving into a region of fluid with a normal velocity, $v_{w}$. At some distance away from the surface the fluid is not affected by the motion of the surface. But Eq. 5.2 results in a normal velocity of $-v_{w}$ for that region of fluid. That is, relative to the surface, fluid far from surface is moving toward the surface at a speed of $v_{w}$. In between the region fully affected by the surface motion and this unaffected region the error is some fraction of $-v_{w}$. Nevertheless, since the most interesting information arising from the normal velocity profile of the boundary layer comes from the flow in the highly affected region, this error does not significantly impact boundary layer analysis.

\subsection{Boundary layer profile analysis}

Once boundary layer profiles, $u(y)$ and $v(y)$, were calculated they were then analyzed automatically to determine boundary layer thickness ( $\delta_{99}$ and $\delta_{95}$ ), wall shear 
stress $\left(\tau_{o}\right)$, tangential velocity at the edge of the boundary layer $\left(U_{e}\right)$ and similarity to known boundary layer solutions. A typical tangential boundary layer profile shows a steep increase in tangential velocity as one moves a short distance from the body surface. Then the tangential velocity reaches some maximum that remains relatively constant for some distance away from the body depending on the surface shape. Therefore, on a tangential profile plot, there is usually a high concentration of points representing particles in this region, moving at this 'exterior' velocity $\left(U_{e}\right)$. The automatic boundary layer analysis code written by the author locates this concentration of points and calculates $U_{e}$. Overlapping vertical strips of the $u$-profile are sampled and the strip with the greatest number of data points is selected. The average velocity of these data points is determined and the strip is re-centered on that average. A new, refined average is calculated from the data points in the re-centered strip and recorded as $U_{e}$. Strip width was set at $10 \%$ of the flume speed for the sequence being analyzed.

Once $U_{e}$ and thickness are known, obvious outliers resulting from errant particle tracks were removed. All velocities greater than $U_{e}$ measured at $<\delta_{95}$ from the surface were assumed to be the result of tracking errors and were removed. Similarly, velocities less than about $80 \%$ of $U_{e}$ and greater than about $120 \%$ of $U_{e}$ at distances $>\delta_{95}$ were also removed. The exact percentages can be optimized by determining the standard deviation in the data used to calculate $U_{e}$ and choosing values representing an envelope of $1-2$ standard deviations. Furthermore, data points above a line running from the point $(u, y)=$ $\left(0,0.2 \delta_{95}\right)$ to the point $(u, y)=\left(0.8 U_{e}, \delta_{95}\right)$ were removed, and points below the line drawn from $(u, y)=\left(0.8 U_{e}, 0\right)$ to $(u, y)=\left(1.2 U_{e}, \delta_{95}\right)$. Once again, the fractions of $U_{e}$ and $\delta_{95}$ used in this filtering process were set based on the quality of the data. Additional erroneous data points were filtered out using the normal velocity profile, removing data points of normal velocities higher and lower than set percentages of $U_{e}$. Since every data point on the normal profile has a corresponding data point on the tangential profile, erroneous data points determined by the several methods above were removed from both the tangential and normal velocity plots to in preparation for further analysis. 
Once outliers were removed, the known boundary layer solutions-Blasius, Falkner-Skan, and the law of the wall, were fit to the data by a least squares fit. It is important to remember that these known boundary layers are observed in steady, flow over flat surfaces with no streamwise pressure gradient present. The boundary layer of a swimming fish does not conform to these conditions, therefore these fits are performed mainly for the interest of comparison. Parameters such as boundary layer thickness and wall shear stress must be determined from the profile data itself and not from the fit profiles. In the fitting process, $U_{e}$ and an initial guess for $\delta_{9 g}$ were taken from the analysis described above. The coefficient of determination, $R^{2}$, was determined from the residual sum of squares and served as a rating for which theoretical boundary layer type the data most resembles. The residual sum of squares was also divided by the number of data points minus the number of fit coefficients to obtain an approximate variance $\left(\sigma^{2}\right)$, and from variance, standard deviation $(\sigma)$.

In addition to the known profiles, a straight line was fit to the tangential velocity profile in the region closest to the body. The slope of this line is taken as an estimated velocity gradient at the surface and is used to estimate the shear stress at the surface. Data points with $u<0.5 U_{e}$ were used. The errors involved in this treatment are dealt with in Chapter 6. $R^{2}$ was calculated for the linear fit for comparison with the value of the theoretical profile fits. This shows which theoretical profile best fits the lower regions of the boundary layer.

In practice, Blasius, law of the law, and linear fits were each preformed twice. Once constraining the curve to intersect with the origin $(0,0)$ and once allowing the $y$ intercept to change. The latter is to allow for the possibility that slight errors were made in determining the exact location of the body surface. $R^{2}$, standard deviation, and the limits of expected error can then be used to determine if such errors are a factor. If so the 
surface location can simply be shifted by the amount suggested by the $y$-intercept of the fit.

The average and normal gradient of normal velocity were calculated to determine if flow was moving in or out of the boundary layer, suggesting acceleration or deceleration of tangential flow in the boundary layer. This information is important since acceleration is a sign of a favorable pressure gradient. Favorable in that it generally results in the boundary layer remaining attached to the surface, whereas a decelerating boundary layer is more susceptible to separation.

\subsection{The relative, local drag coefficient}

As in the preliminary experiments, common fluid parameters such as Reynolds number and coefficients of friction were calculated for each analyzed boundary layer profile. However, it was necessary to develop a new parameter to facilitate the proper comparison of local skin friction on fish. Local coefficients of friction, $C_{f x}$, for a surface such as a flat plate, are usually plotted as a function of the length Reynolds number, $R e_{x}=$ $U x / v$. Regardless of the absolute flow speed, $U$, viscosity, $v$, and distance from the leading edge, $x$, as long as the ratio $U x / v$ is the same, $C_{f x}$ converges to one of two values depending on whether the flow is laminar of turbulent for the same surface. This is most likely not the case for swimming fish. Body undulation and body shape are expected to cause boundary layer parameters such as local friction and external velocity, $U_{e}$, to be much more sensitive to relative body position, $x / L$. It is easy enough to come up with a scaling for a parameter such as $U_{e}$ that makes sense in a plot vs. $x / L$. The first possibility that comes to mind is scaling by the flume speed, $U$. The plots of $U_{e} / U$ for any fish swimming at any speed can be plotted on the same axes and meaningful comparisons can be made. However, the scaling for local friction is not inherently obvious. If an average wall shear stress over the entire fish surface were known, local wall shear stress could possibly be scaled by the average. Since average wall shear stress over the entire fish is a 
difficult value to determine, and since the drag on swimming fish is frequently reported with respect to the drag on a flat plate, the following scaling is proposed and was used to compare local friction as a function of $x / L$ on a flat plate, rigid fish, and swimming fish. The scaled parameter will be referred to as 'the relative, local coefficient of friction', $C_{f x R}$, and is defined as follows,

$$
C_{f x R} \equiv \frac{C_{f x}-C_{f \times B}}{C_{f x T}-C_{f x B}} ; \quad \mathrm{Re}_{x}>4 \times 10^{4}
$$

where $C_{f x}$ is the local coefficient of friction as defined in the preliminary experiments as

$$
C_{f x} \equiv \frac{\tau_{a}}{\frac{1}{2} \rho U^{2}}
$$

and $C_{f x B}$ and $C_{f x T}$ are the local coefficients of friction expected at $x$ if the object were a flat plate with a laminar and turbulent boundary layer, respectively. The restriction of $R e_{x}>4 \times 10^{4}$ is because the theoretical curves of $C_{f x B}$ and $C_{f x T}$ cross at this point. Most of the $R e_{x}$ here fall above this value. The equations for $C_{f x B}$ and $C_{f x T}$ are the same as Eq. 5.4, where $\tau_{0}$ for laminar and turbulent boundary layers are estimated by Eqs. 2.3 and 2.10. Thus, a relative, local coefficient of friction, $C_{f x R}$, of 0 , at any point on a body indicates that the local friction on the body is the same as expected at the same $x$ position on a flat plate with a laminar (Blasius) boundary layer. A $C_{f x R}$ value of 1 indicates local friction equal to that on a flat plate with a turbulent boundary layer $\left(1 / 7^{\text {th }}\right.$ power profile). Values lower than 0 and higher than 1 represent local friction lower and higher than laminar flat plate and turbulent flat plate friction respectively. Using this scaling, local friction on different fish and fish swimming at different speeds can be meaningfully compared, especially when plotted as a function of relative position, $x / L$. 


\subsection{Errors in the calculation of boundary layer parameters}

Errors in the calculation of boundary layer parameters such as $\delta_{99}, U_{e}$, and the tangential velocity gradient at the surface are treated in detail using profiles from the flat plate in Chapter 6.

\subsection{Criteria for accurate boundary layer data}

As mentioned earlier, only image pairs exhibiting well converged surface tracking were analyzed further to produce boundary layer profiles. This tended to restrict measurements that were confined to positions on the fish where the body surface was essentially perpendicular to the laser sheet. In addition, the position of the laser sheet on the fish was known, and data away from the centerline was excluded when necessary due to body curvature.

\subsection{Undulatory phase}

Undulatory phase was determined much more accurately in the current investigation as compared to the preliminary experiments due to the addition of the nearfield camera. In preliminary experiments, undulatory phase was determined by fitting a sine curve to the body velocity, as available, from body motion observed in the small field of view of the boundary layer camera. The wide-angle view of the nearfield camera allowed for very precise measurements of the transverse position of the fish surface at the streamwise position of the field of view of the boundary layer camera. Sampling rate was $15 \mathrm{~Hz}$, much higher than the undulatory frequencies observed $(<6$ $\mathrm{Hz}$ ). The records of transverse position showed long periods of steady swimming with nearly constant frequency at each swimming speed, and relatively constant amplitude at 
fixed positions along the fish (Fig. 5.18). Frequency and phase were determined for every image of a swimming sequence by a least-squares fit of a sine wave to the transverse body motion taking two complete cycles of the body motion at a time. The frequency and amplitude from each two-cycle fit were then used as starting estimates for a refined fit of three overlapping periods in the two-cycle period. Phase and frequency values for each image frame were then given a confidence score on the basis of number of points used for the refined fit and distance from the endpoints of the given fit. The confidence score was then used to restrict data plotted on graphs of boundary layer parameters as a function of phase, or on simple plots such as body amplitude as a function of relative position, $x / L$. 


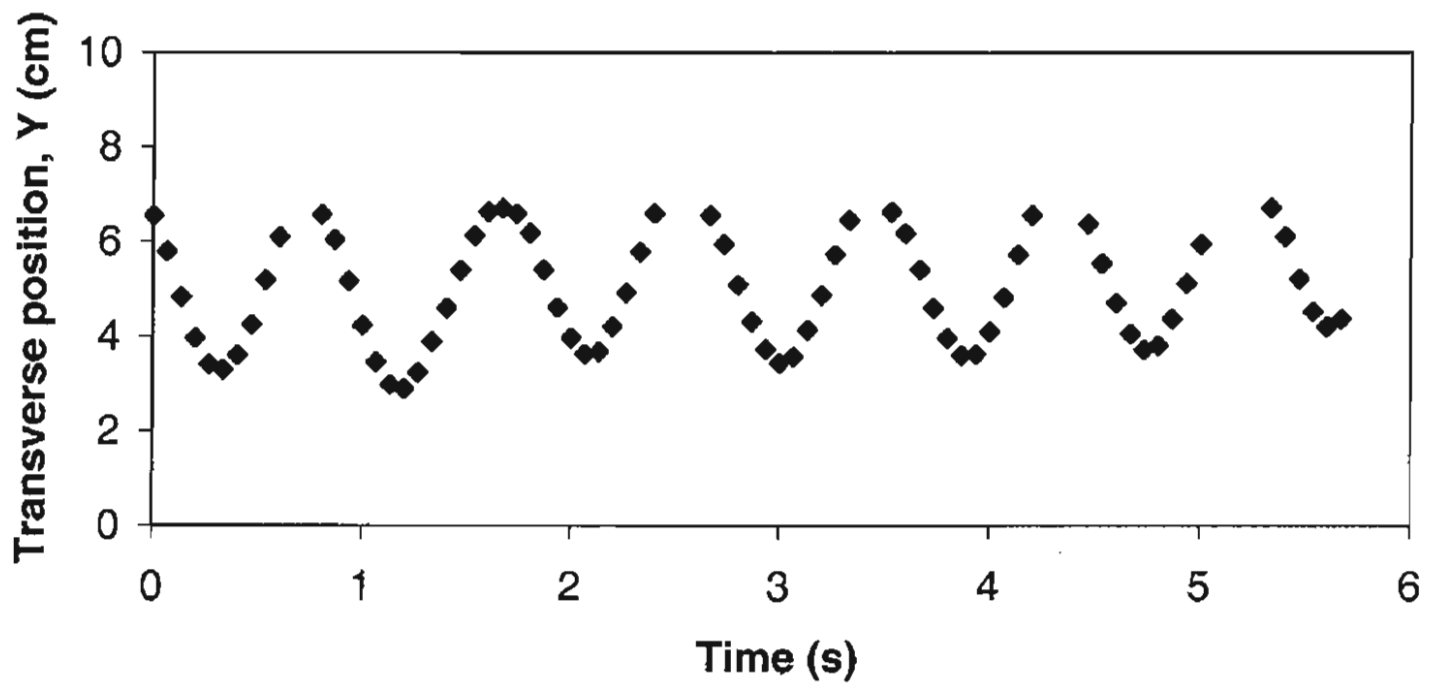

Fig. 5.18 Sample of transverse body surface position in the nearfield camera as a function of time for a bluefish swimming $24 \mathrm{~cm} / \mathrm{s}$.

Occasional dropout occurs due to lack of boundary layer data in the boundary layer camera or problems locating the body surface. 
Page 143 


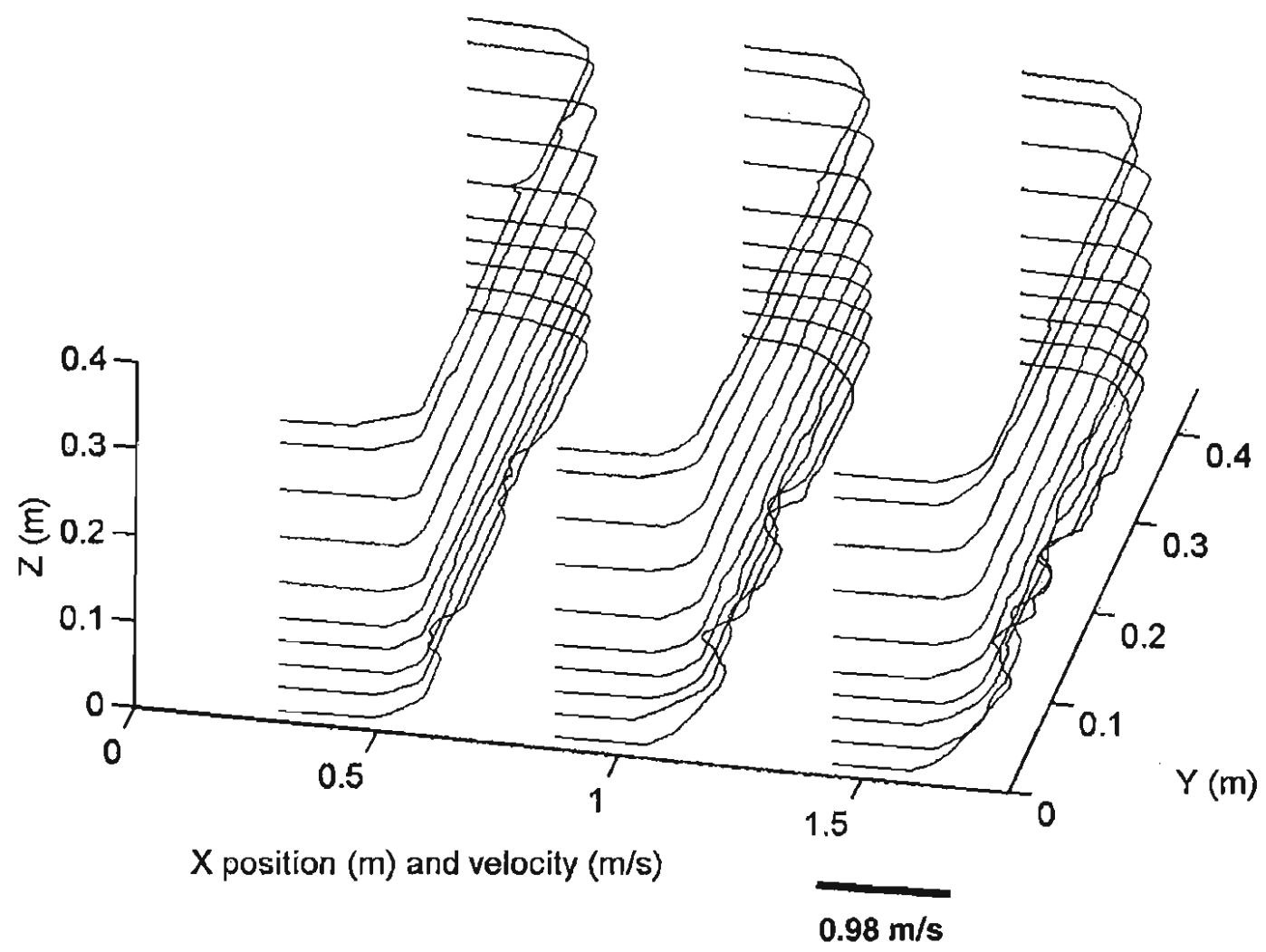

Fig. 6.1 Three streamwise profiles of the test-section flow for the open channe] arrangement, blank inlet barrier, $T=20 \mathrm{C}$, and $U=98 \mathrm{~cm} / \mathrm{s}$. Note the highly uniform flow in the plug and thin boundary layers. Two diagonal/streamwise disturbances converge in the bottom boundary layer. 


\section{Chapter 6}

\section{Experimental controls: flume profile, flat plate, rigid fish}

As described in Chapter 4, three sets of experimental controls were run to serve as reference points for and tests of the experimental procedure. These involved (1) an examination of the flow in the flume test section in the absence of any object, (2) the visualization of the boundary layer flow over a flat plate, and (3) the visualization of the boundary layer flow over fish stretched straight in the flow, i.e. the rigid-body case. This chapter presents the results of these experiments.

\subsection{Flume profile}

Flow in the flume test section was measured with no fish present for each inlet barrier type at three speeds $(23 \mathrm{~cm} / \mathrm{s}, 58 \mathrm{~cm} / \mathrm{s}$ and $98 \mathrm{~cm} / \mathrm{s})$, two temperatures $(15 \mathrm{C}$ and $20 \mathrm{C}$ ), and two test section configurations--open and closed channel. These conditions were a good representation of the range of conditions during experiments. Three streamwise positions were intensively profiled (Fig. 6.1), and will be referred to here as positions $X_{1}, X_{2}$ and $X_{3} . X_{1}=0.30 \mathrm{~m}, X_{2}=0.87 \mathrm{~m}$ and $X_{3}=1.44 \mathrm{~m}$, as measured from the test section inlet. These represent relative positions of approximately $1 / 6,1 / 2$ and $5 / 6$ of the length of the test section. The positions were chosen to obtain a profile near the inlet and outlet of the test section and at the mid-length position. At each $X$-position, the flow at $9-10$ heights above the flume bottom was visualized over the entire cross-stream width of the test section, $Y=0$ to $45 \mathrm{~cm}$. This was performed using 5 overlapping, $10 \mathrm{~cm}$ $x 10 \mathrm{~cm}$ horizontal planes of visualization in the cross-stream direction. The resulting cross-stream profile resolution was $3-4 \mathrm{~mm}$, just small enough to examine the wavelength of fluctuations in the mean cross-stream profile caused by the inlet barriers. The $X, Y$ and $Z$ positions of all desired horizontal planes to be visualized were fed to the 
robots via a simple text file. The PC controlling the robot communicated with a PC controlling the image acquisition so that 3 images of the flow were taken at each of the 135 to 150 flume positions. Communication was mediated by a TTL pulse (5V) sent from one of the analog output channels on the NI-7344 motion controller card to one of the analog input channels on a National Instruments, PCI-6024E data acquisition board on the image acquiring PC. The arrival of the pulse was used as a trigger in software to initiate the acquisition of three images. After a sufficient wait time, the robots moved to the next position and repeated the process. Three images were taken to insure that at least one image pair with the desired time step, $\Delta t$, was acquired. Time steps used were 4 $\mathrm{ms}$ at the slow flume speed and $2.1 \mathrm{~ms}$ for the medium and fast speeds observed. All possible image pairs were processed by a hybrid DPIV code developed by S. McKenna as described in McKenna and McGillis (2002) and the proper pairs were easily selected by comparing correlation scores. Three-dimensional profiles of the flume as in Fig. 6.1 were then constructed from the $X, Y$, and $Z$ coordinates of the robot program. Four characteristics of the flow were examined, (1) the thickness of the wall boundary layers, (2) the average speed of the flow outside of the wall boundary layers, i.e. the plug flow, and (3) the root-mean-squared (RMS) variation of the streamwise profile of the plug (an estimate of turbulence intensity). Each was examined as a function of streamwise position in the flume while comparing the effects of temperature, inlet barrier, channel type and the flume speed setting.

\subsubsection{Flume wall boundary layers}

Three to four wall boundary layers occur in the flume test section depending on the configuration, i.e. whether open or closed channel. These are the two side-wall boundary layers, the bottom boundary layer, and, in the closed channel case, the ceiling boundary layer. In every trial, the side-wall boundary layers were found to be thinner than the bottom boundary layer. The side-wall boundary layers were nearly identical in thickness, growing from $1.8 \mathrm{~cm}$ to $3.2 \mathrm{~cm}$ from position $X_{1}$ to $X_{3}$ with a $95 \%$ confidence 
interval, $C I_{95}$, in the mean of $0.3 \mathrm{~cm}$, and a standard deviation, $\sigma$, of $0.7 \mathrm{~cm}$. The bottom boundary layer grew from $3.8 \mathrm{~cm}$ to $7.0 \mathrm{~cm}\left(C_{95}=0.5 \mathrm{~cm}, \sigma=1.2 \mathrm{~cm}\right)$. In the case of closed channel flow (depth $=25 \mathrm{~cm}$ ), the 'ceiling' boundary layer was found to grow from $1.0 \mathrm{~cm}$ to $3.2 \mathrm{~cm}\left(C_{95}=0.2 \mathrm{~cm}, \sigma=0.6 \mathrm{~cm}\right)$. These differences in thickness are not surprising considering the configuration of the flume. The contraction upstream of the test section is most pronounced and symmetric in the horizontal (Fig. 4.2). Thus one would expect similar and thin boundary layers on the side-walls. The thin, yet rapidly growing boundary layer of the ceiling in the closed channel configuration (over 300\% from $X_{1}$ to $X_{3}$ ), can be understood by the fact that the ceiling was a flat plate of acrylic, with a $10^{\circ}$ bevel on the leading edge. Boundary layer growth begins at the leading edge, rather than upstream on the walls of the flume. The growth rate on the ceiling agreed exceptionally well with that of a $1 / 7^{\text {th }}$-power, flat plate turbulent boundary layer profile in all but two cases-no barrier and the medium mesh barrier at the lowest speed $(23 \mathrm{~cm} / \mathrm{s})$ where laminar flow might be expected. Both cases showed increased rate of growth in the second half of the flume, suggesting a transition to turbulence. In fact, the flume wall boundary layers in general exhibited thickness and growth characteristic of turbulent boundary layers in the majority of cases.

The only other exception to these findings occurred occasionally on the side-walls at the higher speeds of the open channel case. The boundary layer was about $0.6-1.6 \mathrm{~cm}$ thinner than in the closed channel at the position $X_{3}$. Interestingly, the bottom boundary layer showed the opposite effect, but the differences in thickness were small and barely significant. Despite the reduced growth rate in the side-wall boundary layer, its thickness would have required a $14 \mathrm{~m}$ lead distance to have been the result of flat plate laminar boundary layer development. Of course, the configuration of the flume upstream of the test section was not a flat plate and may account for such growth, even for laminar flow. The contraction ratio of the flume is $6: 1$, and therefore flow upstream of the contraction is $1 / 6$ times the speed in the test section. Thus laminar boundary layer growth in the precontraction region could be significant. Laminar flat plate boundary layers grow as 
$(1 / U)^{1 / 2}$ and $X^{1 / 2}$ (Eq. 2.3), while turbulent boundary layers grow as $(1 / U)^{1 / 5}$ and $X^{4 / 5}$ (Eq. 2.10). Therefore laminar boundary layer growth is more sensitive to $U$ than turbulent boundary layer growth, and turbulent boundary layer growth is more sensitive to $X$. For example, at a fixed position $X$, if velocity, $U$, is divided by 4 , a laminar boundary layer would be 2 times the thickness at the original velocity, whereas a turbulent boundary layer would be only 1.32 times its thickness at the slower flow speed. However, at a fixed flow velocity, a laminar boundary layer at $4 X$ is 2 times its thickness at $X$, whereas a turbulent boundary layer at $4 X$ is $\sim 3$ times its thickness at $X$. That is, turbulent flat plate boundary layers grow at a greater rate as a function of distance.

The pre-contraction region of the flume is roughly $2 \mathrm{~m}$ long including a series of flow-straightening arrays. Using Blasius laminar and $1 / 7^{\text {th }}$-power turbulent flat plate boundary layer theories for rough approximations of potential growth of the precontraction boundary layer, predicted thicknesses are $1.8-3.7 \mathrm{~cm}$ for laminar flow and $6.0-8.0 \mathrm{~cm}$ for turbulent flow over the range of flume speeds observed. The lower ends of these ranges occur at the highest flume speed setting observed in the flume profile experiments (i.e. $98 \mathrm{~cm} / \mathrm{s}$ in the test section). The boundary layer may be slightly thicker than these estimates, since the initial boundary layer arises from a stagnation flow with a thickness $>0$, different from flat plate theory. In the contraction, the boundary layer is expected to thin. Recall that the test section boundary layer thickness at position $X_{I}$ for the side-walls averaged $1.8 \mathrm{~cm}$. For laminar growth, this suggests a thickness of about $1.7 \mathrm{~cm}$ at the inlet. This value is lower than the predicted pre-contraction thickness given above for laminar flow $(1.8-3.7 \mathrm{~cm})$. Therefore, considering thinning during the contraction, the boundary layer just prior to the inlet may possibly have been laminar, and the slow growth observed in the open channel configuration at medium to high flume speeds could be evidence that side-wall boundary layers occasionally remained laminar. If the boundary layer at the inlet had been thicker than $3.7 \mathrm{~cm}$ it would be difficult to argue that the inlet boundary layer was laminar. Nevertheless, other factors, besides the laminar vs. turbulent nature of the boundary layer can influence the growth of boundary 
layers. For example, a favorable pressure gradient can result in slow growth, while an adverse pressure gradient can lead to high growth rates.

Inlet barrier types and flume speed did not exhibit large effects on wall boundary layers. There was some hint that boundary layers were slightly thicker for the finest mesh barrier and the lowest flume speed, but the effects were barely significant. These observations, especially the weak effect of flume speed on boundary layer thickness, point to the conclusion that in the overwhelming majority of cases, the wall boundary layers in the flume test section were turbulent. $R e_{x}$ ranged from $1 \times 10^{5}$ and $1.4 \times 10^{6}$ at the $X$ positions examined if measured from the inlet. The range includes the typical transitional values- $-3.5 \times 10^{5}$ to $5 \times 10^{5}$. Furthermore, the actual range of $R e_{x}$ is certainly higher than that determined above in which the inlet was treated as $x=0$, since boundary layers were already developing upstream of the inlet (except for the ceiling). Transition was likely encouraged at the inlet due to the joint between the contraction region and the test section. At the joint, there is a recessed slot about $1.5 \mathrm{~cm}$ wide for a stainless steel frame used to support the netting of the inlet barrier. Although the tolerances are small and the frame does not completely fill the slot, the non-uniformities in the surface at the inlet joint would be expected to encourage transition to turbulence. Flume profiles taken with a blank frame did not show significant differences in boundary layer thickness, suggesting further that the joint and not the mesh barriers, had the greater impact on the flow. As mentioned earlier, the ceiling boundary layer in the closed channel arrangement showed some agreement with laminar boundary layer theory in two cases at the lowest speed $(23 \mathrm{~cm} / \mathrm{s})$. Since one of the laminar-like cases of the ceiling boundary layer occurred for the medium mesh grid it is not clear that the simple presence of a barrier was enough to induce a turbulent boundary layer. Finally; the profiles of flow in the test section, when viewed in 3-D reconstruction (Fig. 6.1) suggest two spiral vorticies in the bottom boundary layer pealing off the bottom corners of the inlet joint and converging as they are swept downstream. This may help to explain the thicker bottom boundary layer. 
In spite of these finding, it is not expected that the wall boundary layers had a significant impact on the fish and flat plate boundary layer data, except in the case of the live eel. The flat plate and rigid fish were profiled at a position $15.5 \mathrm{~cm}$ off the bottom, $26.5 \mathrm{~cm}$ from the front wall, and $28.5 \mathrm{~cm}$ from the back wall, well within the plug flow of the flume. In live fish experiments, data was not taken if fish were within about $10 \mathrm{~cm}$ of the front side-wall, and body thickness alone made it unlikely that the back side-wall affected the boundary layer measurements, which were taken on the side of the fish facing the front side-wall. Bluefish, mackerel and scup tended to swim high enough off the bottom and far enough from the ceiling or free surface that neither the wall boundary layers or free surface effects were expected to impact the measurements. Eel, on the other hand, nearly always swam within $2-4 \mathrm{~cm}$ of the bottom, and were usually within the bottom boundary layer. Nevertheless, the analyses of the boundary layers of swimming fish performed here include case-by-case determination of the tangential velocity at the outer edge of the fish boundary layer, $U_{e}$. Therefore, regardless of the depth at which eel swam, the approximate flow speed experienced by the eel was known. Measurements in the bottom boundary layer may be quite applicable to the swimming of eel in situ, such as in the case of a tidal stream.

\subsubsection{Flume plug flow}

Average flow speed in the flume outside of the wall boundary layers was not significantly affected by temperature or the inlet barriers. Once again the most significant effect was that of the channel arrangement. Flow in the closed channel was 3 - 5\% higher than in the open channel case at the same flume speed setting (as measured in rotational frequency of the motor driving the flume impeller). This is not surprising since in the closed channel case the flat plate 'ceiling' blocks about $4 \%$ of the flume cross-sectional area and there is an additional wall boundary layer, which can be thought of as a partial blockage of flow. Another effect observed in the closed channel configuration was a $1-4 \%$ increase in flume speed from $X_{1}$ to $X_{3}$. The larger increases 
occurred at higher speeds. The open channel arrangement showed $<1 \%$ increase in flume speed over the same region. Flume speed increase with streamwise position is a normal effect due to the growth of boundary layers and incompressibility. The volume flow through the inlet and outlet must be the same, therefore larger boundary layers (i.e. more slowed flow) near the outlet require plug flow to increase. Recall that in the previous section (6.1.1) it was reported that side-wall boundary layers were observed to grow much faster in the closed channel arrangement at medium and high speeds. This, as well as the growth of an additional boundary layer (ceiling) in the closed configuration explains the greater increase in streamwise plug flow in the closed channel case. In most cases, these increases were on the order of 0.5 to $2 \mathrm{~cm} / \mathrm{s}$ per meter of test section and are not expected to have had any impact on the investigation. As mentioned before, flow speed at the outer edge of each fish boundary layer was measured independently for each boundary layer realization.

The average magnitude of cross-stream flow, $V$, in the flume plug for all flume speed settings and experimental conditions was $<2.2 \%\left(C_{95}=0.3 ; \sigma=0.4\right)$ of the average streamwise velocity of the flume, $U$. The local directions and magnitudes of the cross-stream flow revealed a combination of two well-known phenomena. First, the wall boundary layer growth and streamwise increase in $U$ observed in the flume test section would lead a student of fluid dynamics to predict cross-stream flow directed away from the walls and toward the center of the flume. In order for wall boundary layers to thicken and for plug flow to increase, fluid must be transferred from the boundary layers to the plug. Surprisingly, the cross-stream flume profiles showed that this was only the case over about the lower $1 / 3$ of the flume depth. Above this point, the cross-stream flow was directed toward the walls away from the center, but the magnitudes were only about $1 / 2$ of those in the lower $1 / 3$ of the flume. In actuality, the $V$ magnitude was greatest at the bottom of the flume, decreased gradually to 0 at the $1 / 3$ position, and then increased to the top of the flume, but with direction switched. This suggests that there is a slight circulatory flow directed upward at the center of the flume and downward along the 
sides. This kind of flow is reminiscent of a phenomenon in pipe flow known as secondary flow, which occurs at bends in a pipe. Secondary flow in the center plane of a bend moves away from the inside curve of the bend, while flow near the walls moves toward the inside curve. The result is a pair of streamwise vorticies. The vertical asymmetry in the flume contraction may result in a similar situation. There is a bend on the bottom of the contraction, but the top of the contraction is flat (Fig. 4.2), therefore the fluid experiences some of the same sort of forcing that fluid in a pipe bend experiences and secondary flow would be in the direction suggested by the $V$-profiles of the flume test section. This flow might also be expected to convect faster moving fluid closer to the side-walls in the upper $2 / 3$ of the flume, thereby potentially causing the boundary layer to be thinner near the top. A second look at the side-wall boundary layers showed this to be the case. The superposition of the two effects-flow toward the center due to boundary layer growth and circulatory flow due to secondary flow-would be expected to lead to the relatively higher magnitudes in cross-stream flow in the lower $1 / 3$ of the test section and the relatively lower magnitudes in the upper test section. Secondary flows are known to be common in non-circular channels even without channel bending (Schetz, 1993), but the asymmetry in $V$ with depth, $Z$, in the flume suggests the contraction 'bend' dominates in the production of the secondary flow observed here. In spite of these findings, recall that the relative velocities represented in these phenomena are on average less than $2.2 \%$ of the streamwise plug flow. Therefore, they are not believed to have significantly impacted the findings of this investigation.

\subsubsection{Fluctuations of streamwise velocity in the plug flow}

Two types of fluctuations in streamwise velocity within the plug flow were examined, (1) the fluctuation in the $U$-profile with respect to the $Y$-direction, $u_{x y}^{\prime}$, and (2) the fluctuation in $U$ along the $X$-direction, $u_{x x}^{\prime}$. The latter is determined from the fluctuations in each $X$-direction row of vectors in the PIV realizations of the flow at each of the 135 to 150 flume positions visualized, and is akin to the streamwise turbulent 
fluctuations. The former value simply rates the uniformity of the flume profile, not the turbulence. There was not enough resolution to properly determine $u_{x z}^{\prime}$, but it is not expected to be significantly different from $u_{x y}^{\prime}$. The root-mean-squared (RMS) values of the fluctuations as a percentage of the average streamwise velocity, $U$, were calculated. These values tell one how 'quiet' or uniform is the flow. The power spectrum of $u_{x y}^{\prime}$ for each profile was also calculated to determine if the inlet barrier grid spacing affected the flow in a coherent way.

With no inlet barrier, the average values RMS of $u^{\prime} x y$ at $X_{l}, X_{2}$ and $X_{3}$ were $1 \%$, $1 \%$ and $1.5 \%$ of $U\left(C I_{95}=0.2 ; \sigma=0.3\right)$, respectively, for the full set of temperatures, channel arrangements and flume speed settings. Therefore, fluctuations tend to increase very slightly over the second half of the test section. The values are fairly consistent with the flume manufacturer's claim of $1 \%$ for which no streamwise distribution was given. With barriers in place, the values were in a ' $v$ '-shaped pattern vs. position, $1.5 \%, 1.2 \%$ and $1.5 \%\left(C I_{95}=0.2 ; \sigma=0.4\right)$ at $X_{1}, X_{2}$ and $X_{3}$, respectively. Therefore, the barriers appear to increase fluctuations at the inlet, and the tendency is for these fluctuations to be damped out in the first half of the test section. This effect can be seen in 3-D flume profiles, especially at $98 \mathrm{~cm} / \mathrm{s}$ with the large grid size (Fig. 6.2). However, looking at each case separately, lower speeds and smaller grid size resembled the pattern of gradual increase of the blank inlet, while higher speeds and larger grid sizes tended toward the ' $v$ '-shaped pattern of the averages with the barriers in place. In general, profile fluctuations were lower in the closed channel arrangement, but only by about $0.2 \%$ of $U$. The power spectra of the profiles (Fig. 6.3) showed that the dominant fluctuation wavelength in the profiles at $X_{I}$ was precisely the distance between the vertical strands of the grid barrier. The blank inlet case showed no obvious dominant wavelength.

The fluctuation of $U$ in the $X$-direction, $u^{\prime} x x$ with a blank inlet was $1.2 \%, 1.2 \%$, and $1.3 \%\left(C l_{95}=0.4 ; \sigma=0.7\right)$ at $X_{1}, X_{2}$, and $X_{3}$, respectively whereas with barriers in place the value at $X_{I}$ was $1.5 \%$ and dropped back to about $1.2 \%$ at $X_{2}$, remaining there 


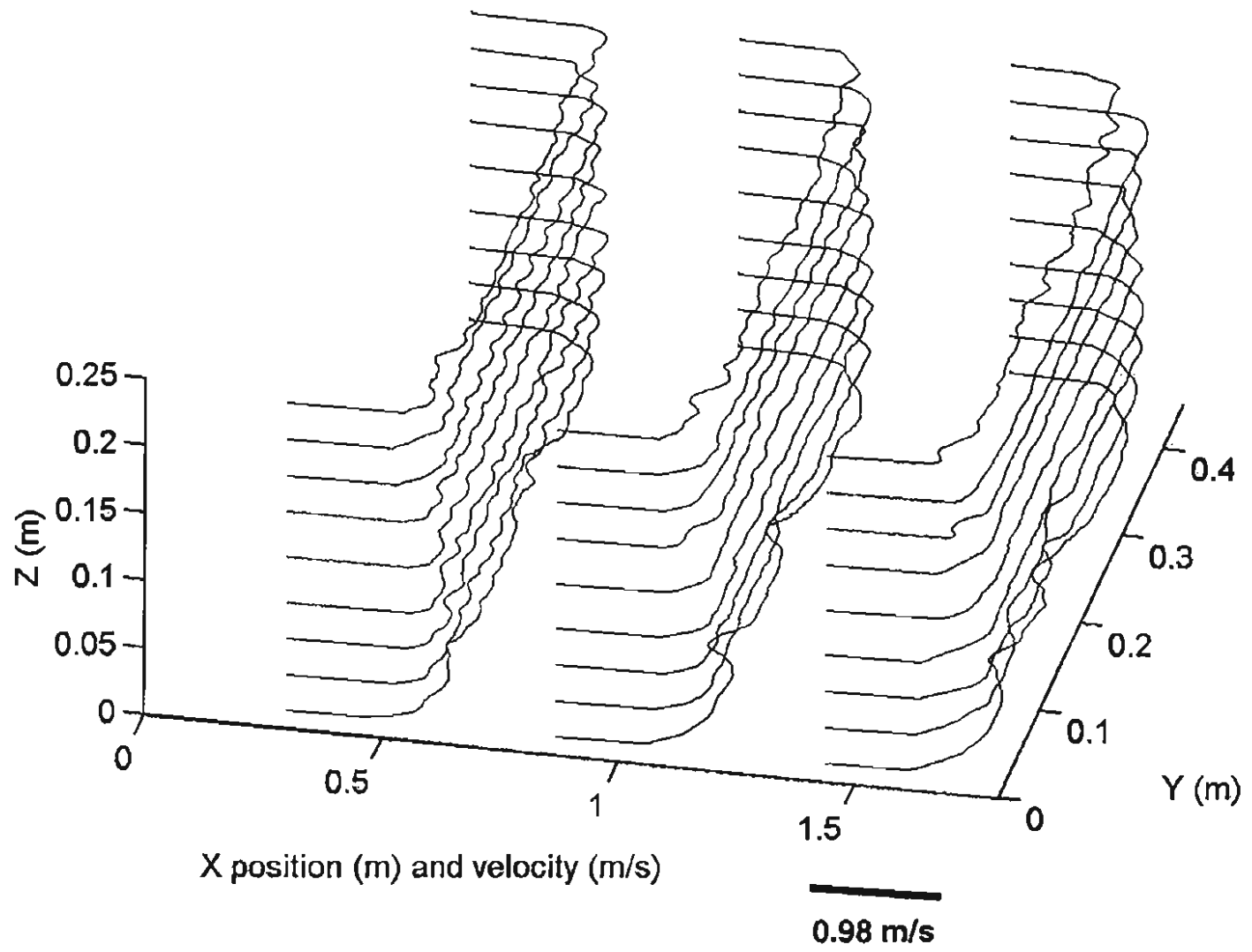

Fig. 6.2 Three streamwise profiles of the test-section flow for the open channel arrangement, large grid size inlet barrier $(3.0 \mathrm{~cm} \mathrm{x} 3.7 \mathrm{~cm}), T=20 \mathrm{C}$, and $U=98$ $\mathrm{cm} / \mathrm{s}$. Note how the profile fluctuations with respect to the $Y$-direction caused by the inlet barrier are gradually damped out. Compare to the flume profile at the same speed with a blank inlet barrier (Fig. 6.1). 

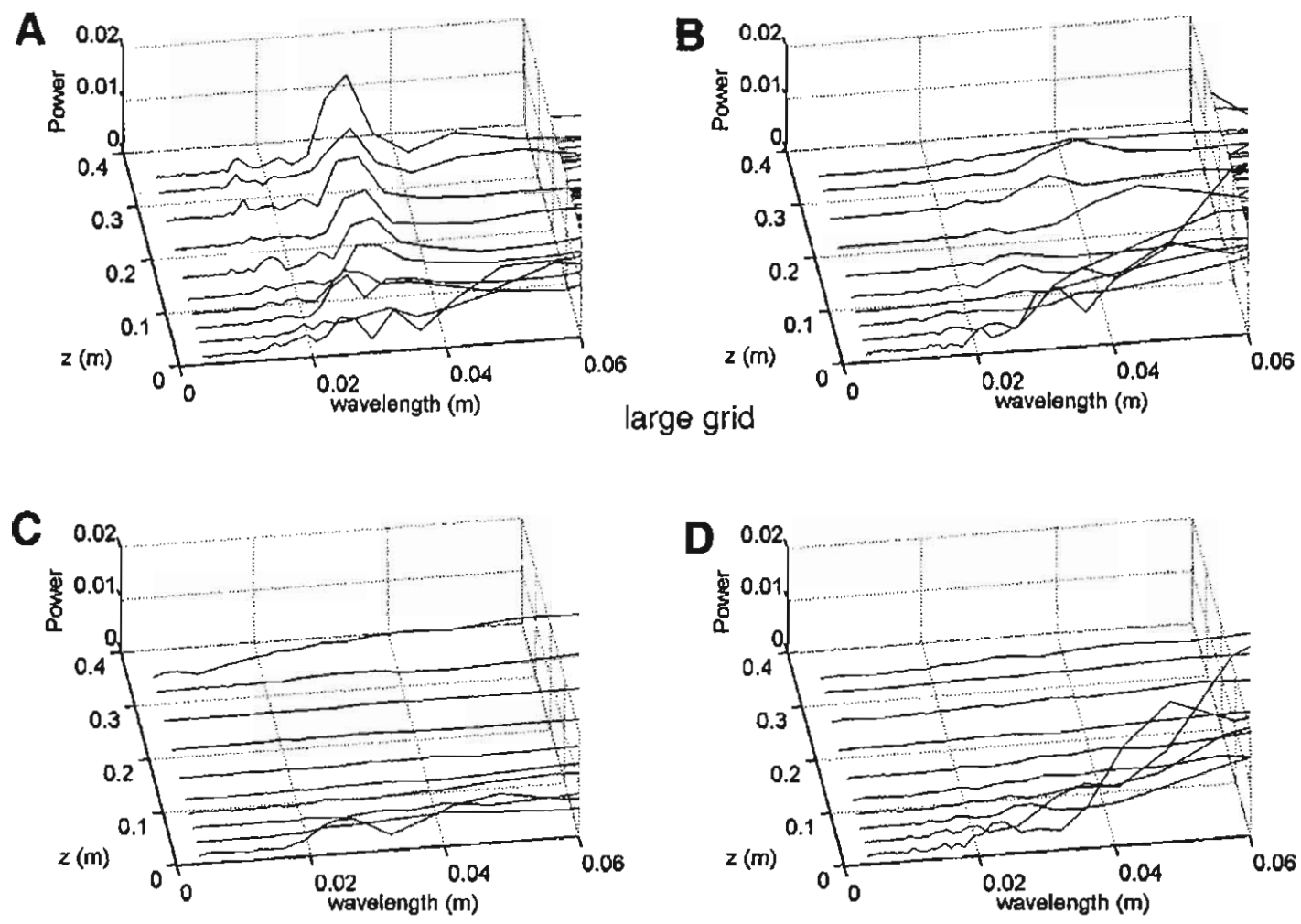

$$
\mathrm{X}=\mathrm{X}_{1}
$$

blank grid

$X=X_{3}$

Fig. 6.3 Power spectra of fluctuations in flume $U$-profiles with respect to the $Y$ direction at each depth, $Z$, for $X=X_{1}$ and $X_{3}(U=98 \mathrm{~cm} / \mathrm{s}$, open channel arrangement, $T=20 \mathrm{C}$ ). These are the same profiles shown in Figs. 6.1 and 6.2. $(A, B)$ Large grid size inlet barrier $(3.0 \mathrm{~cm} \times 3.7 \mathrm{~cm})$. (C, D) Blank inlet barrier. Plot (A) shows that the dominant wavelength near the inlet $\left(X_{I}\right)$ is the spacing of the horizontal grid spacing (i.e. $3.0 \mathrm{~cm}$ ). Plot (B) shows that fluctuations of this wavelength are damped out by the time flow nears the outlet $\left(X_{3}\right)$. No comparable dominant wavelength is seen in (C) or (D). Fluctuations in the bottom boundary layer (i.e. $Z$ near 0 ) are similar for both barrier types. 
through $X_{3}\left(C I_{95}=0.2 ; \sigma=0.4\right)$. A closer look showed that at $X_{1}$ the fluctuations were only $1 / 3$ to $1 / 2$ times the average value of $1.5 \%$ downstream of the spaces in the grid, while directly downstream of the grid strands the fluctuations were higher than the average. This suggests that the flow between the grid strands remained relatively laminar for a long distance while each strand was followed by a slightly turbulent wake. Inspection of the individual flow field realizations near $X_{I}$ confirmed this (Fig. 6.4). The structure was often recognizable at $X_{2}$ and an occasional hint of the pattern was seen at $X_{3}$. The structure was more stable for larger grid size and slower speed.

\subsubsection{Summary of flume profile analysis}

Although the preceding analysis of wall boundary layers, plug flow velocity and velocity fluctuations suggests that flow in the flume test section is complicated and nonuniform, it is important to consider the magnitudes of the effects described relative to the average plug flow. Overall, the non-uniformity in the plug flow was shown to be very small, and is not expected to have had a significant impact on the results of this investigation, even in the presence of the inlet barrier grids. The fluctuations in the flow are about $1 / 4$ to $1 / 2$ times those measured in the test section used in the preliminary study, and even there, laminar-like boundary layers on swimming fish were frequently observed. That is, turbulence caused by upstream barriers did not dictate the presence of a turbulent boundary layer on swimming fish. The same was observed in this investigation (see Chapter 7). Certainly the fluctuations in the plug flow would be expected to cause an earlier transition to a turbulent boundary layer than for quieter flow, but it is easy to imagine that fish experience turbulence intensities of equal or greater magnitudes in the wild. These findings, however, do point out the measurable impact of flume design on test section flow and the importance of characterizing test section flows in experimental fluid dynamics. The manufacturer of the flume used in this investigation (Engineering Laboratory Design, Inc.) indicates that the contraction of the flume is symmetrical in cross-section and has analytically determined contours, however, the 


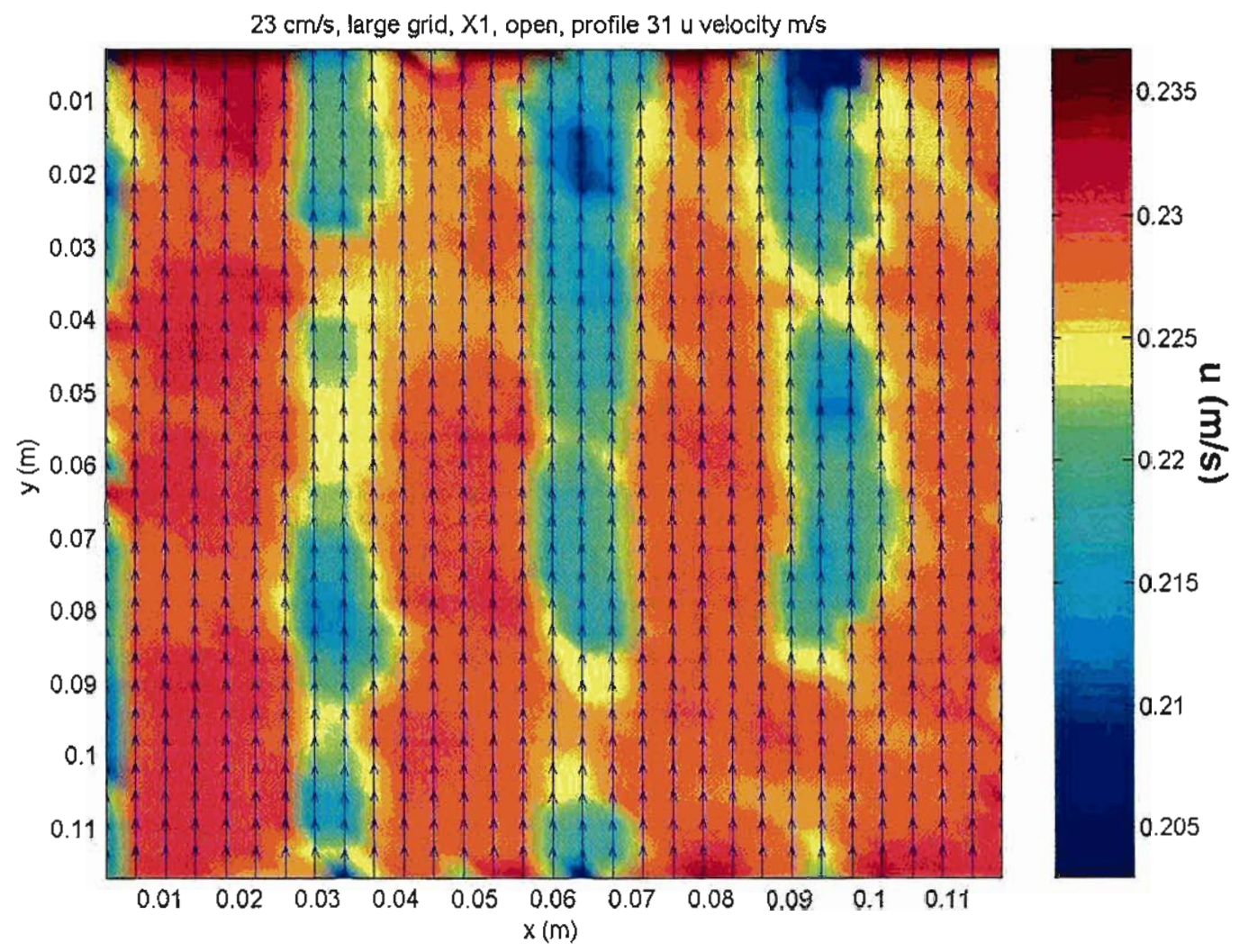

Fig. 6.4 u component of velocity field near the inlet $\left(X_{l}\right)$ with the large grid inlet barrier $(U=23 \mathrm{~cm} / \mathrm{s}$, open channel arrangement, $T=20 \mathrm{C})$. The vertical stripes of slower, more turbulent flow are the wakes following the grid strands. 
symmetry is only in the cross-stream direction and the inlet joint is problematic. The result is a small secondary flow, a thicker than ideal bottom boundary layer, and streamwise vorticies in the bottom boundary layer. These effects could certainly bias more sensitive sorts of experiments, such as attempting to measure the vertical or crossstream locomotion of very small organisms, such as copepods, in a horizontal current.

\subsection{Flat plate boundary layer}

The boundary layer over a flat plate was profiled in 56 trials covering all inlet barrier types, a wide range of flume speeds, and two temperatures. Profiles from individual image pairs and ensemble averages of profiles at several positions along the plate were used to investigate boundary layer development, the character of the flow, wall shear stress, and statistical uncertainties in the measured values. Both long and short time-series of boundary layer profiles were used in the ensemble averages to examine fluctuations in local profiles. These trials were conducted to test the automatic boundary layer profiler developed in this investigation and to examine the impact of the experimental conditions on boundary layer flow. The flat plate is a classic fluid dynamics problem, which has been extensively described (Schlichting, 1979; Schetz, 1993).

\subsubsection{Details of the flat plate experiments}

The flat plate investigated was $38.5 \mathrm{~cm}$ in streamwise length and about $60 \mathrm{~cm}$ wide. It was constructed from a sheet of $3 / 16$ "opaque white acrylic and the leading and trailing edges were beveled at $10^{\circ}$ on the 'back' side of the plate, i.e. opposite to the side profiled. The plate was oriented vertically spanning from the bottom of the flume, through the free-surface, to a beam running parallel to the $X$-direction of the flume. Water depth was $45 \mathrm{~cm}$. The plate was clamped to the beam so that the bottom edge of the plate was pinned against the bottom of the flume. Three small rubber feet $(2 \mathrm{~mm}$ 
thick) were stuck to the bottom edge of the plate and prevent the plate from sliding. As mentioned earlier, the boundary layer was visualized at a position $15.5 \mathrm{~cm}$ off the bottom, $26.5 \mathrm{~cm}$ from the front wall, and the leading edge was $43 \mathrm{~cm}$ from the inlet of the test section. Therefore the results were taken in the plug of the flume flow.

The boundary layer over the flat plate was imaged at 13 positions taking 150 images at each position. The flow $10 \mathrm{~cm}$ upstream from the plate was also profiled. Two temperatures $(15$ and $20 \mathrm{C})$, seven flume speeds $(14.4-117 \mathrm{~cm} / \mathrm{s})$, and all four inlet barrier configurations (blank; small, medium, and large grid) where examined. Short and long time-series average profiles were constructed for each position on the plate. In the case of short time-series, 20 consecutive images were processed by the automatic profiling code at each plate position. This resulted in 10 profiles, the data points (i.e. particle velocity components) of which, were simply plotted together and analyzed as an ensemble average. The long time-series were constructed by processing every 10 th image pair, and combining the resulting 7 boundary layer profiles. In all, a total of 12,376 flat plate boundary layer profiles were determined and 952 upstream profiles. Profiles from individual image pairs were also analyzed to estimate the statistical uncertainties in boundary layer profiling by the methods used in this investigation. Fig. 6.5 shows the development of the boundary layer over the flat plate for the case of the blank inlet barrier at a flume speed of $33.0 \mathrm{~cm} / \mathrm{s}$. Note the highly converged data, suggesting very regular laminar boundary layers. Boundary layer growth is very similar to Blasius except near the trailing edge of the plate. Inflow evident in the v-profile near the trailing edge indicates that the boundary layer is accelerating, thus explaining the decrease in $\delta_{9 g}$ in that region. Fig. 6.6 shows the boundary layer for the same configuration at $U=68.7 \mathrm{~cm} / \mathrm{s}$. Boundary layer thickness follows even closer to Blasius and signs of acceleration of that boundary layer near the trailing edge are negligible. 

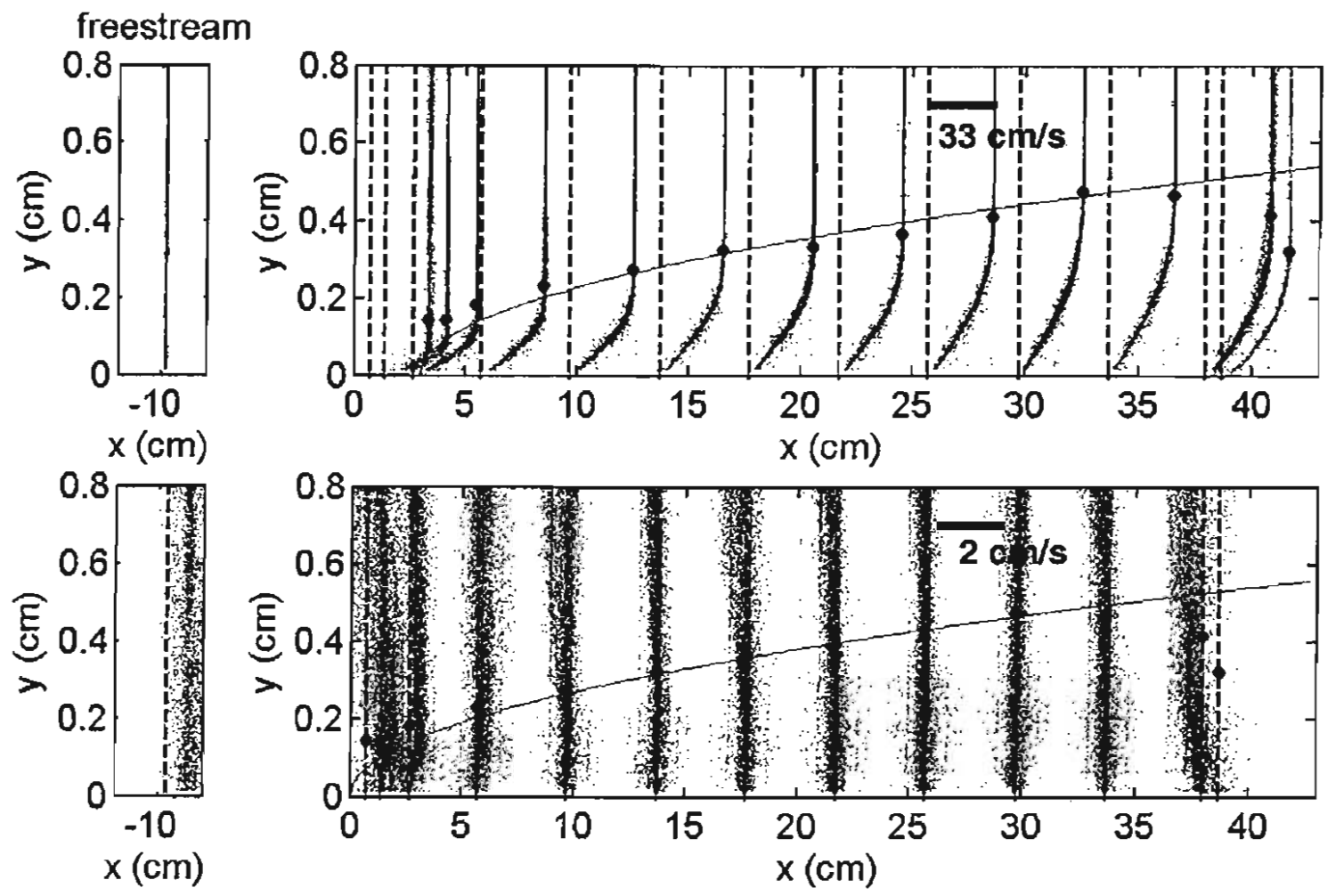

Fig. 6.5 Flat plate boundary layer, $u$ - and v-profiles with freestream profile $(x=$ $10 \mathrm{~cm}$ ) for $U=33.0 \mathrm{~cm} / \mathrm{s}$ and the blank inlet barrier,. Top row is $u$-profile, bottom row is $v$-profile. Short time-series, $T=20 \mathrm{C}$. Dotted vertical axes are $u=$ 0 (top) and $v=0$ (bottom). Filled circles indicate boundary layer thickness, $\delta_{g 9}$. Solid curves indicate $\delta_{99}$ from Blasius. Note that scales are different for $u$ and $v$. Negative values in the v-profile near the trailing edge indicate an accelerating boundary layer, which exhibits the typical concomitant decrease in $\delta_{99}$. 

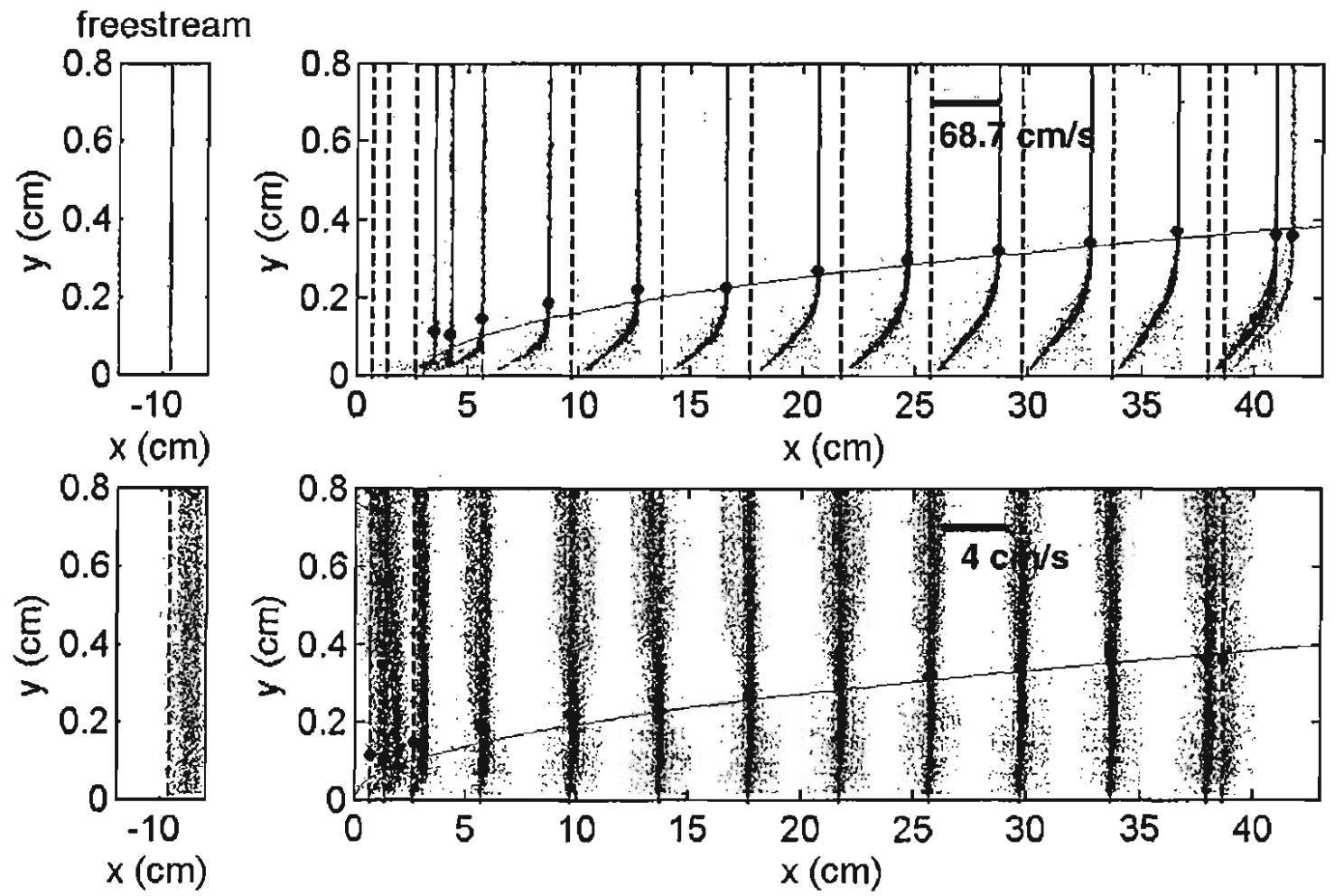

Fig. 6.6 Flat plate boundary layer, $u$ - and v-profiles with freestream profile $(x=$ $-10 \mathrm{~cm}$ ) for $U=68.7 \mathrm{~cm} / \mathrm{s}$ and the blank inlet barrier. The profiles show that the boundary layer is more similar to the Blasius boundary layer near the trailing edge than the profiles for $U=33.0 \mathrm{~cm} / \mathrm{s}$ (Fig. 6.5). The v-profiles do not exhibit the acceleration seen at the lower speed. Top row is u-profile, bottom row is $v-$ profile. Short time-series, $T=20 \mathrm{C}$. Dotted vertical axes are $u=0$ (top) and $\mathrm{v}=$ 0 (bottom). Filled circles indicate boundary layer thickness, $\delta_{99}$. Solid curves indicate $\delta_{99}$ from Blasius. 


\subsubsection{The flat plate coordinate system}

A coordinate system, $x y z$, different from the flume coordinate system and attached to the flat plate is used in the following discussion concerning the flat plate boundary layer. It is the same as the coordinate system used in the discussion of boundary layer theory in Chapter 2. The origin is at the leading edge of the plate in the plane of the laser. The $x$-axis is tangent to the surface of the flat plate in the streamwise direction and represents distance from the leading edge. It is parallel to the $X$-axis of the flume, but only because the plate is flat and oriented streamwise. The same coordinate system is used for rigid and swimming fish, and since the surfaces are curved, the tangent, or direction of $x$, is not always parallel to $X$. The $y$-axis is the outward normal of the plate and represents distance from the surface. Due to the experimental configuration the $y$ axis is parallel to the $Y$-axis of the flume, but in the opposite direction. Again, in the case of fish, the outward normal is not always parallel to $Y$. The $z$-axis is tangent to the plate and perpendicular to $x$. It is the cross-stream direction for the flat plate. Since the flow was essentially illuminated in the $x y$-plane alone, no quantitative information concerning cross-stream flow is presented here. Velocities in the $x, y$, and $z$ directions will be referred to as $u, v$, and $w$.

\subsubsection{Comparison of upstream profile with flume plug profile}

Before taking a closer look at the boundary layers over the flat plate, it is instructive to examine the profile of the flow $10 \mathrm{~cm}$ upstream of the flat plate $(x=-10$ $\mathrm{cm}$ ) (Fig. 5.11) and compare it to the plug flow profiled by DPIV (section 6.1). The upstream profiles were determined using the boundary layer profiling code simply treating the edge of the image as a 'pseudo-surface'. In this way, the PTCV code developed in this investigation can be also used to characterize general flows. Quadratic regression of the RMS of $u_{x x}^{\prime}$ from the flume plug analysis predicts that the value at $x=-$ 
$10 \mathrm{~cm}$ for the open channel case should be $1.2 \%$ of $U\left(C l_{95}=0.5 ; \sigma=0.5\right)$ with the blank barrier and $1.6 \%\left(C I_{95}=0.2 ; \sigma=0.3\right)$ for grid barriers. The values determined from upstream profiles were $1.0 \%$ of $U\left(C I_{95}=0.3 ; \sigma=0.5\right)$ with no inlet barrier and $1.4 \%$ $\left(C I_{95}=0.1 ; \sigma=0.3\right)$ with barriers present. That is, from a statistical position the values are essentially the same and have similar uncertainty when measured by either technique. Furthermore, the fluctuations at $x=-10 \mathrm{~cm}$ were observed to be slightly larger for the largest grid size as was observed in the plug flow analysis. Most importantly, these numbers suggest that velocities measured using the particle tracking code developed here are at least as accurate as those measured by conventional DPIV code.

Accuracy in particle tracking is generally limited by two factors: (1) matching particles from image to image and (2) locating the centroid of particles. Improper matching of particles usually leads to large errors, on the order of the average nearestneighbor distance between particles in the field of view. For example, consider an image pair in which particles are about 20 pixels apart and traveling about 50 pixels per frame. Assuming an errant match always involves a nearest neighbor of the appropriate match, errors in distance traveled are $+/-40 \%$, and as travel distances decrease, relative error increases. This is why conventional DPTV codes work best when restrictions are placed on allowable track distance and angle. In fact, conventional codes prefer lower particle densities, which therefore relax the restrictions that have to be placed on allowable angle and distance since the likelihood of a neighbor (i.e. an errant match) meeting the restrictions is reduced. Of course, lower particle densities mean less flow resolution, and therefore random errant tracks due to nearest neighbors impact more heavily on the data. By contrast, the code developed in this work reduces nearest neighbor errors by looking at the motions of sets of particles. This works best at higher particle densities, and the effect of errant matches is diluted, but more significantly, only errant matches that are similar to other local matches are produced and errors are less widely distributed. The resolution of the upstream profile velocity fluctuations discussed above suggests that this particle-matching algorithm is working exceptionally well. This can be confirmed by 
inspection of superimposed image pairs with overlaid particle tracks (Fig. 5.11, 5.13, $5.15)$.

Locating the centroids of particles introduces errors on the order of the diameters of particles. Since, particle diameters are typically much smaller than the average distance between nearest-neighbors, the resulting errors are smaller. Errors in determining centroids tend to be normally distributed and therefore uncertainty decreases with larger samples of particle tracks. A typical particle diameter in this investigation was about 4 pixels. For a particle traveling 50 pixels, the maximum expected error due to locating particle centroids would be $8 \%$, however finding the center of intensity of the particle or taking the maximum of a Gaussian surface fit has been shown to result in subpixel accuracy. Errors on the order of 1 pixel or less translate to about $1-2 \%$ in the example used here. They are most likely the dominant source of error, however small, in the particle tracking code developed in this investigation.

Closer inspection of the upstream profile reinforces this conclusion about of the accuracy of the particle tracking code. The values of RMS in $u^{\prime}{ }_{x x}$ given above are the result of analyzing ensemble average profiles. If the variance of $u_{x x}^{\prime}$ in ensemble profiles is representative of the variance at all times across the entire plug, then a single profile should have the same RMS value, i.e. the same standard deviation. The confidence intervals should be larger because the number of particle tracks sampled decreases. However, this was not found to be the case. For example, the ensemble average of 10 sequential profiles taken upstream of the flat plate at a flume speed of $33.0 \mathrm{~cm} / \mathrm{s}$ (blank inlet barrier) (Fig. 6.5) had an RMS of $u_{x x}^{\prime}$ of $1.05 \%$ of $U$, with $\left(C I_{95}=0.03 ; \sigma=1.1\right)$. Taking an individual profile from the ensemble, one finds that the RMS of $u_{x x}^{\prime}$ actually decreases to just $0.43 \%$ of $U$, with $C I_{95}=0.04 ; \sigma=0.4$, that is, the smaller sample is significantly less scattered. Sample size decreased by an order of magnitude from $n=$ 4654 particle tracks to $n=463$ particle tracks. In such a case, if the variance, $\sigma^{2}$, in the data were constant, the width of the confidence interval would have more than tripled and 
the standard deviation, $\sigma$, would have stayed the same. Instead the confidence interval barely increased and the standard deviation was more than cut in half. For all of the blank inlet barrier cases, the RMS in $u_{x x}^{\prime}$ for all individual profiles with $n>100$ averaged $0.70 \%$ of $U$, with $C_{95}=0.07$ and $\sigma=0.7$. These findings demonstrate (1) that in the upstream profile, the variance of $u_{x x}^{\prime}$ is significantly lower than for the ensemble profiles, and (2) that the potential level of accuracy of the particle tracking code is even higher than predicted by comparing the flume plug profiles to the upstream profile ensemble averages. Stated simply, if having significantly more data points (i.e. ensemble profiles) leads to increased uncertainty in the data, then the dominant source of variance is likely the data and not the measuring device. That is, there are slight fluctuations of the mean freestream flow $(<1 \%)$ with time scales shorter than the time-series examined here.

The average magnitude of $Y$-direction flow, $V$, in the upstream profiles was $2.4 \%$ of $U$. In the analysis of the flume plug flow the average magnitude of $V$ was found to be $<2.2 \%$. The slightly higher value in the upstream profile is due to the fact $V$ in the flume plug fluctuated above and below zero with position, $Y$. Therefore the average was influenced by low values where the profile passed through $V=0$. The profile upstream of the flat plate was in a region where $V$ was always positive (i.e. toward the back wall of the flume) and non-zero. Therefore the average $V$ in that region was higher than the average over the entire plug. The direction and magnitude of the flow in the upstream profile agreed with the plug flow analysis at the same position. Recall that in the coordinate system of the flat plate, flow toward the back wall of the flume ( $+Y$-direction) is in the $-y$-direction (toward the plate). Nevertheless, even at the first position on the plate, the $y$-direction velocity is directed away from the plate as the boundary layer begins to grow (Fig. 6.5). 


\subsubsection{General observations of the flat plate boundary layer}

The majority of the flat plate trials were characterized by highly converged, Blasius-like tangential boundary layer profiles ( $u$-profiles) that gradually grew in thickness over the length of the plate (Figs. 6.5, 6.6, top row). Fig. 5.13 shows an individual boundary layer profile at $x=21.3 \mathrm{~cm}$ for the boundary layer shown in Fig. 6.5. In general, boundary layers grew slightly slower than predicted by laminar flat plate theory at the slowest flume speeds. As speeds increased, boundary layer thickness decreased and converged more and more to Blasius. At the highest speed, $117 \mathrm{~cm} / \mathrm{s}$, there was clear evidence of a transition to a turbulent boundary layer. Fig. 6.7 shows the case of $U=117 \mathrm{~cm} / \mathrm{s}$ and the blank inlet barrier. Profiles over the first half of the plate still appear to be laminar in profile shape, data convergence and growth rate. However, after $x=20 \mathrm{~cm}$, the boundary layer growth increases significantly and the profiles show signs of particle tracking difficulties. These are discussed below. Fig. 6.8 shows a sample profile from this region of the flat plate. The profile is more similar to the law of the wall than Blasius. Inlet barrier type had a small, but measurable impact on boundary layer thickness, and apparently played a role in encouraging transition at the highest flume speed (Fig. 6.9). Temperature and time scale of sampling had very small to negligible effects on the measured boundary layer. Boundary layer thickness is examined in more detail below.

The scatter in the data of Figs. 6.7 and 6.9 is due largely to errant particle matches. This occurs for two reasons. First, in turbulent flow, high shear and threedimensional flow result in rapid changes in the patterns of even closely situated particles. Shear causes nearby particles to travel different distances and three-dimensionality causes particles to appear and disappear as they pass in and out of the plane of the laser sheet. Second, the high speed of the flow means that particles travel large distances in the field of view. This results in many particles leaving the field of view and, more significantly, it leads to smaller differences in track distances and angles for the errant tracks to nearest 


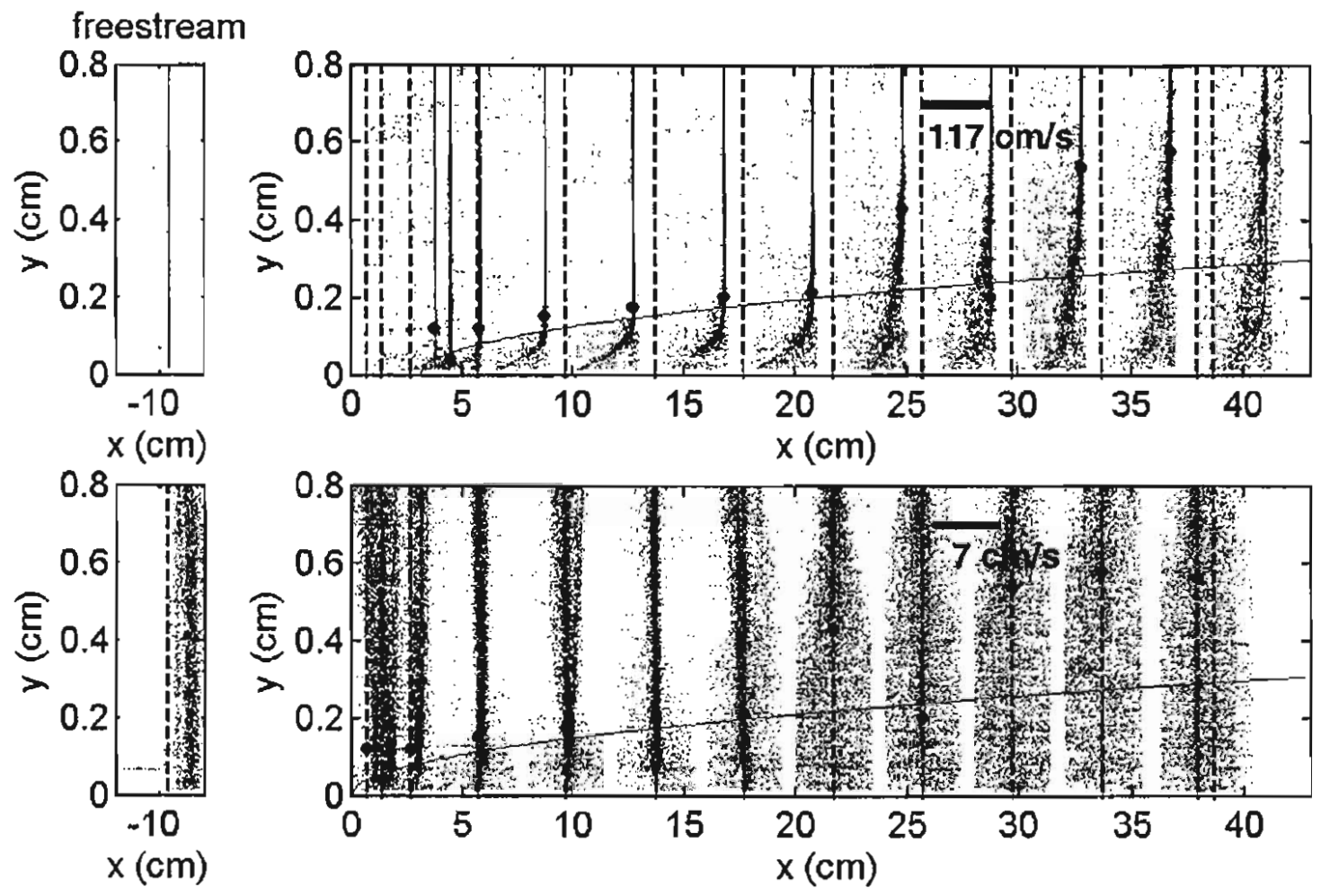

Fig. 6.7 Flat plate boundary layer, $u$ - and v-profiles with freestream profile $(x=$ $-10 \mathrm{~cm}$ ) for $117 \mathrm{~cm} / \mathrm{s}$ and the blank inlet barrier. The boundary layer behaves and looks laminar for first half of plate. Rapid growth and profile shape over the second half of the plate indicates a transition to turbulent flow. Particle tracking difficulties at this high speed resulted in considerable scatter in the profiles, which is the cause of the poorly determined $\delta_{g g}$ of the early profiles and the profile at $x=26 \mathrm{~cm}$. The long time-series trial with the same conditions showed $\delta_{9 g}$ to be near $0.5 \mathrm{~cm}$ at the latter position, as inspection and the trend suggest. Top row is $u$-profile, bottom row is v-profile. Short time-series, $T=20 \mathrm{C}$. Dotted vertical axes are $u=0$ (top) and $v=0$ (bottom). Filled circles indicate boundary layer thickness, $\delta_{9 g}$. Solid curves indicate $\delta_{9 g}$ from Blasius. 

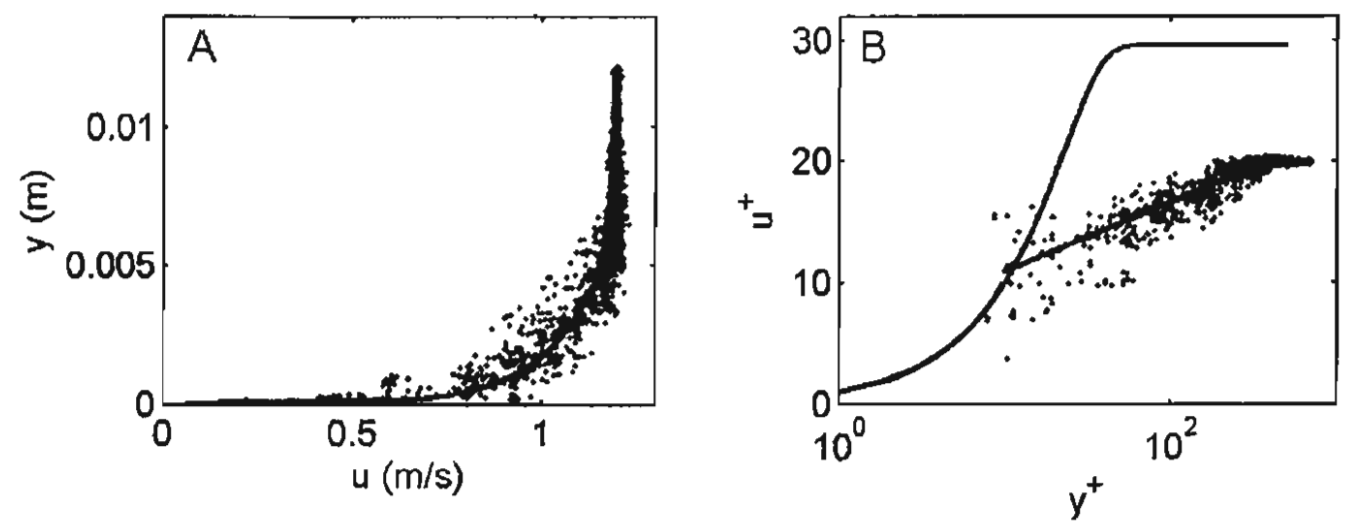

Fig. 6.8 Ensemble $u$-profile (blue points) at $x=29.4 \mathrm{~cm}$ of the flat plate boundary layer shown in Fig. 6.7. (A) standard plot of $y$ vs. $u$ fit to the law of the wall (black curve); (B) the same profile using scaling common to the law of the wall with the fit to the law of the wall (black) and Blasius (red). Inlet barrier is blank and $U=117 \mathrm{~cm} / \mathrm{s}$. Scatter in the profile was manually removed leaving only the dense band of data representing the true profile shape. 

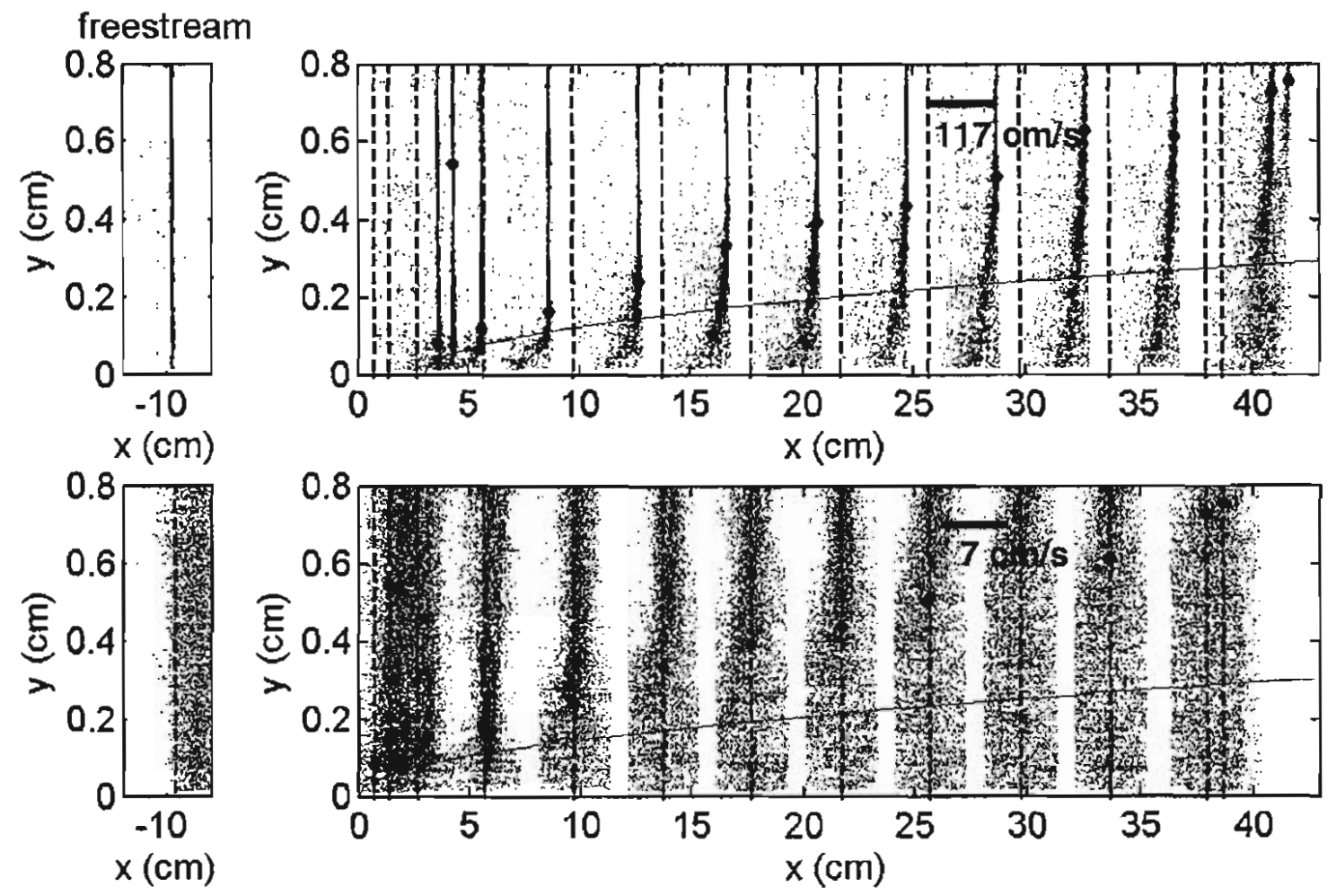

Fig. 6.9 Flat plate boundary layer, $u$-and $v$-profiles with freestream profile $(x=$ $-10 \mathrm{~cm}$ ) for $U=117 \mathrm{~cm} / \mathrm{s}$ and the small grid inlet barrier. The profiles demonstrate the early transition to turbulence caused by the presence of an inlet barrier grid compared to the blank inlet barrier (Fig. 6.7). Top row is $u$-profile, bottom row is v-profile. Short time-series, $T=20 \mathrm{C}$. Dotted vertical axes are $u$ $=0$ (top) and $v=0$ (bottom). Filled circles indicate boundary layer thickness, $\delta_{99}$. Solid curves indicate $\delta_{99}$ from Blasius. The obviously errant $\delta_{99}$ at $x=4 \mathrm{~cm}$ was due to scatter as explained in Fig. 6.7. 
neighbors relative to the correct track. Thus the uncertainty in choosing correct tracks increases. The obvious solution to these problems is to decrease the time between exposures. In this investigation, the smallest possible time difference between exposures was limited to $2.1 \mathrm{~ms}$ and was used in the case of $U=117 \mathrm{~cm} / \mathrm{s}$. This leads to particle travel of $0.25 \mathrm{~cm}$ in the $1.35 \mathrm{~cm}$ field of view or a distance of about 180 pixels. For optimum particle tracking by the code developed in this investigation, a travel distance of less than 100 was desired. Wernet's (1993) code prefers even shorter particle travel, less than about 20 pixels. Particles in the boundary layer do travel slower than the freestream flow, but for turbulent profiles, the velocity gradient near the surface is so great, that flow throughout the majority of the boundary layer is generally more than $0.5 U$. Nevertheless, theoretical profile shapes are distinguishable within the scatter of the flat plate profiles at $117 \mathrm{~cm} / \mathrm{s}$, once again illustrating the power of the particle tracking algorithm developed here. The profiles over the first half of the plate are clearly laminar-like and, as will be show in the next section, the turbulent profiles of the second half of the plate resemble the profiles for the 'law of the wall.' The growth of the boundary layer also agrees with the $1 / 7^{\text {th }}$-power turbulent boundary layer approximation. This will also be dealt with in detail below. Length Reynolds number, $R e_{x}$, at the position of transition was $1 \times 10^{5}$ to 3 $\times 10^{5}$. Van Driest and Blumer (1963) determined an equation for the transition of a flat plate boundary layer based on the freestream turbulence intensity. Their equation predicts a transition $\operatorname{Re}_{x}$ of $2.5 \times 10^{5}$ to $5.0 \times 10^{5}$ using the turbulence intensities determined from the plug flow analysis and the upstream profile $(1.0-1.5 \%)$. This is very good agreement considering the simple construction of the acrylic flat plate used in this investigation.

\subsubsection{Boundary layer thickness}

Maximum boundary layer thickness over the flat plate ranged from about 3.5 $8.0 \mathrm{~mm}$ as flume speed was decreased from $94.1-14.4 \mathrm{~cm} / \mathrm{s}$, respectively. At $117 \mathrm{~cm} / \mathrm{s}$ the maximum thickness grew to about $9.0 \mathrm{~mm}$ due to the transition to a turbulent 
boundary layer. Boundary layer thickness as a function of distance from the leading edge, $x$, for all flat plate experiments was fit to the function type,

$$
\delta(x)=\beta_{o} x^{\beta_{1}}
$$

by linear regression, where $\beta_{v}$ and $\beta_{1}$ are the regression coefficients. Intervals for $95 \%$ confidence were determined during the regression. This was performed for all 315 applicable combinations of the flat plate experimental variables ( 7 speeds, 4 inlet barriers + all experimental barriers, 2 temperatures + all temperatures, 2 time series + all time series). Eq. 6.1 was chosen because the Blasius boundary layer and $1 / 7^{\text {th }}$-power turbulent profile are known to grow as $\mathrm{x}^{0.5}$ and $\mathrm{x}^{0.8}$, respectively. The coefficient of determination, $R^{2}$, for the 315 regressions had a mean value of 0.89 . Only 24 regressions had $R^{2}$ values lower than 0.70 and they were all at the two highest flume speeds, reflecting the impact of large particle travel distances and turbulence at high speed flow.

As mentioned above, laminar boundary layer profiles were observed at all speeds, including the front half of the plate for some of the trials at $117 \mathrm{~cm} / \mathrm{s}$. For flume speeds of $14.4-68.7 \mathrm{~cm} / \mathrm{s}$ the boundary layer grew as $x^{0.33}$, and at $94.1 \mathrm{~cm} / \mathrm{s}$ the growth was as $x^{0.39}$, somewhat lower than predicted by Blasius. For the highest flume speed, the boundary layer grew as $\mathrm{x}^{0.69}$ with grid-type inlet barriers and $\mathrm{x}^{0.42}$ with the blank inlet barrier. The high growth rate with the grid-type barriers is indicative of the earlier transition to turbulence compared to cases with the blank barrier (Figs. 6.7, 6.9). In fact, since the growth rate at transition increases, the growth rate coefficients determined for the highest flume speeds do not reflect the true growth rate in the turbulent region on the plate. The slow growth in the first half of the plate 'pulls' the fit down. The fact that the growth rates were often slightly smaller than laminar and turbulent theory are likely due to the fact that flat plate theory assumes a streamwise pressure gradient of zero and an infinite extent of the flat plate and freestream flow. A negative streamwise pressure gradient would be expected to cause boundary layer thinning. A slight negative angle of 
attack of the plate could also result in some boundary layer thinning, but the images, which show the surface of the plate, suggest that this was not the case. The flat plate in this investigation was relatively short in the streamwise direction and the flume test section is not an unbounded flow. Boundary layer growth sometimes appeared to slow near the trailing edge of the plate (Fig. 6.5).

In order to analyze more thoroughly the possible effects of barrier types, flume speed, temperature and sampling time, the regression of $\delta_{95}$ vs. $x$ for each flat plate case was compared to every other applicable case to determine which cases, if any, exhibited significant differences in boundary layer thickness. An example of a non-applicable case is comparing the effect of the small inlet barrier to all experimental barriers, since the small barrier is a member of the second grouping. In all, 11,480 comparisons were made between the flat plate cases. Of those, 2054 showed no significant difference in boundary layer thickness. 9,426 comparisons showed a significant difference, but 9,250 of those were from cases of different speeds. Therefore, excepting the effect of flume speed, only 176 of 11,480 comparisons showed significant differences in boundary layer growth.

Close inspection revealed that the 176 comparisons were in three groups. First, there were 6 comparisons that suggested that the boundary layer was $0.1-0.2 \mathrm{~mm}$ thicker at $20 \mathrm{C}$ than at $15 \mathrm{C}$ over the second half of the plate, but only at $U=68.7 \mathrm{~cm} / \mathrm{s}$ and involving the blank inlet barrier. This is in the face of hundreds of comparisons showing no significant impact of temperature, including 39 other comparisons involving $U=68.7 \mathrm{~cm} / \mathrm{s}$ with a blank barrier. A look at all 2054 comparisons deemed nonsignificant shows that this first grouping is most likely an artifact. The non-significant comparisons showed average differences in boundary layer thickness of $0.2-0.3 \mathrm{~mm}$, with an average $R^{2}$ of 0.86 . The average $R^{2}$ value of the first grouping was 0.97 . Due to the large sample size of flat plate cases $(n=315)$ and the general goodness of fit of Eq. 6.1 to boundary layer growth, it is statistically expected that in some cases, $\delta_{95}$ vs. $x$ will be fit exceptionally well. In fact, the $95 \%$ prediction interval for $R^{2}$ for all of the 
comparisons extended above 1 , which is the $R^{2}$ value of a perfect fit. A high $R^{2}$ means a very narrow confidence interval. A comparison of two cases with very narrow confidence intervals has a very good chance of showing a significantly difference. The fact that the observed difference $(0.1-0.2 \mathrm{~mm})$ is in the range of the non-significantly different cases, and that the frequency of the occurrence was low and unsupported by similar cases, suggests the first set is a random artifact.

The second group of cases showing significantly different boundary layer growth contains 109 comparisons all involving the inlet barrier with the small grid size at $U=$ $21.8-68.7 \mathrm{~cm} / \mathrm{s}$. The grouping suggests that at these medium speeds, the small grid often results in a boundary layer that is $0.3-0.4 \mathrm{~mm}$ thicker than all other barrier typesmedium, large, and blank--over the second half of the plate. This difference is small considering the $0.2-0.3 \mathrm{~mm}$ differences observed in the non-significant group. In addition, if the effect were consistent, one would expect it to be observed in 300 comparisons rather than 109. Nevertheless, there is some evidence that the small grid size tends to slightly increase boundary layer thickness. This may be related to the earlier finding that flume flow through the larger grid sizes was relatively quiet and stable downstream of the spaces in the grid. Fluctuations directly behind the grid strands contributed the most to the overall fluctuation in streamwise flow. Since there is less strand material per unit area in the larger grids the flow may behave more like the blank frame. No significant difference was observed in boundary layer thickness comparing the blank grid to the medium and large grids. This is certainly the expected result as grid size is increased more and more. On the other hand, the small grid, with more strands per area, that is, more fluctuation-producing structure, is expected to lead to greater overall fluctuation, which may have affected boundary layer growth. However, the analysis of the flume plug showed no significant difference in fluctuations for the three grid sizes.

The third and final grouping from the 176 significant comparisons produced the only substantial effect observed. The group consists of 61 comparisons involving the 
blank inlet barrier at the highest flume speed, $117 \mathrm{~cm} / \mathrm{s}$. The grouping shows that the flat plate boundary layer was $0.9-1.9 \mathrm{~mm}$ thinner with a blank inlet barrier compared to all other barrier types. This is easily understood as evidence of a later transition to a turbulent boundary for the blank barrier due to lower fluctuations in the oncoming flow, as demonstrated earlier in the growth coefficients, $\beta_{l}$, at this speed. The RMS of fluctuations in $U$ in the flume with grid barriers in place was, in general, about 1.5 times greater than for the blank barrier. Therefore, the transitional $R e_{x}$ predicted by the equation of Van Driest and Blumer (1963) is lower for the grid barriers-closer to $1 \mathrm{x}$ $10^{5}$. This is precisely what was observed. By contrast, transition occurred at $R e_{x}=2.6 \mathrm{x}$ $10^{5}$ with no inlet barrier present.

\subsubsection{Errors in estimating velocity gradient at the plate surface}

A more important measured quantity than boundary layer thickness is the velocity gradient, $\partial u / \partial y$, at the surface of the plate. This allows one to calculate the wall shear stress, $\tau_{o}$ and the local coefficient of friction, $C_{f x}$. Of course, it is impossible to determine $\partial u / \partial y$ at the very surface with any particle image velocimetry method since the closest distance measurable is the radius of a particle. However, in both laminar and turbulent flat plate theory $\partial u / \partial y$ becomes linear as one gets sufficiently close to the surface. It is possible to determine estimated errors in $\partial u / \partial y$ at the surface on the basis of the region over which $\partial u / \partial y$ is calculated near the surface. For example, if velocity data is available in the lower $10-30 \%$ of the boundary layer (i.e. $y / \delta=0.1-0.3$ ), the theoretical error in estimating $\partial u / \partial y$ at the surface by a free linear fit for a Blasius profile is only $3.7 \%$. If the fit is constrained to pass through the origin the error is just $1.1 \%$. For a turbulent profile, the errors depend on flow speed since the turbulent profile is not always geometrically similar. Since turbulent profiles were only observed at $117 \mathrm{~cm} / \mathrm{s}$ on the flat plate, this speed will be used. Errors in $\partial u / \partial y$ in this case are $80.4 \%$ for a free linear fit and $18.5 \%$ for a fit constrained to pass through the origin. In the case of Falkner-Skan, over the majority of the range of boundary layer acceleration and deceleration, the errors 
by a free linear fit are $<41 \%$ and errors by a fit constrained to pass through the origin are $<24 \%$. In the worst-case scenario, if the only velocity information one has about the boundary layer is in the upper $95 \%$, the error in $\partial u / \partial y$ for a Blasius boundary layer is only $26 \%$ for a line drawn from the origin to $\delta_{95}$. This is not a large error considering how crude is the approximation. For a Falkner-Skan boundary layer the error is $34-55 \%$ for all but highly inflected boundary layers. For turbulent boundary layers the error is $>$ $94 \%$, and is therefore not an option.

In this investigation, the particle tracking code measured velocities well into the boundary layer, down to 7 to 14 pixels from the surface. At the resolution used here, that translates to near or below $y / \delta=0.1$. In the flat plate experiments, the great majority of boundary layers fit Blasius with $R^{2}$ values from 0.80 to 0.99 . For each individual profile and for ensemble average profiles, errors in the estimation of the $\partial u / \partial y$ as described here were factored into the uncertainty in $\partial u / \partial y$ based on the nearness to the surface of the velocities and the method of fit. For ensemble profiles, a free linear fit was used. On average predicted errors in $\partial u / \partial y$ were $2.8 \%\left(C I_{95}=0.03 ; \sigma=0.5\right)$, which was similar to the average standard deviation in $\partial u / \partial y$ from the fits themselves $\left(3.7 \%, \mathrm{Cl}_{95}=0.3 ; \sigma=\right.$ 3.5). The average $95 \%$ confidence interval for a given linear fit of $\partial u / \partial y$ was $0.7 \%$ of the calculated $\partial u / \partial y$. For individual profiles, average predicted errors in $\partial u / \partial y$ were $3.1 \%$ $\left(C l_{95}=0.02 ; \sigma=0.9\right)$, and the standard deviation in $\partial u / \partial y$ from the fit was $22.3 \%\left(C I_{95}\right.$ $1.7 ; \sigma=74.2$ ). In $84 \%$ of the profiles, $\sigma$ in $\partial u / \partial y$ was less than $20 \%$. The average $95 \%$ confidence interval was $12.2 \%$ of $\partial u / \partial y$. The statistics on the individual profiles give an estimation of the accuracy of the boundary layer profiling system to determine $\partial u / \partial y$ from individual image pairs, as in the live fish work. Since the predicted errors in measuring $\partial u / \partial y$ by a linear fit for the majority of profiles were much smaller than the confidence interval and standard deviation resulting from the regression itself, statistical uncertainties were used to compare plots involving quantities originating from $\partial u / \partial y$ (e.g. $C_{f x}$ ). The uncertainties in the ensemble average profiles were used in the analysis of 
$\partial u / \partial y$ in the flat plate. In the case of turbulent boundary layers, the best fit of the profile using the law of the wall was used. Although this method was discouraged in the preliminary investigation, it is less problematic in the case of a flat plate. Of course, it is not ideal, since pressure gradients could affect profile shape, but it was often found to be the only alternative due to high predicted errors as discussed earlier.

The automatic boundary layer analysis code was successful in determining the flow character in the boundary layer-whether laminar or turbulent- $92 \%$ of the time for the region $x / L=0.15-1.0$ along the flat plate.

\subsubsection{Velocity gradient and local coefficient of friction on the flat plate}

As for boundary layer thickness, all 11,480 applicable comparisons of $2 u / \partial y$ as a function of $x$ were made between flat plate trials and groups of trials. Velocity gradient, $\partial u / \partial y$ was used rather than wall shear stress or the coefficient of friction since it was the measured quantity. The latter quantities have additional built in temperature dependencies due to the presence of viscosity in their definitions. For the temperatures 15 to $20 \mathrm{C}$ there is a $13 \%$ difference in viscosity and therefore using these quantities could result in an artificial significant difference. The velocity gradient, $\partial u / \partial y$, definitively demonstrates whether the experimental conditions had an effect on the boundary layer. The analysis showed that aside from flume speed and the transition to turbulence, no significant differences in $\partial u / \partial y$ vs. $x$ were present. That is, temperature and sample time had no significant impact on $\partial u / \partial$, while inlet barriers only played a role at the highest speeds. The transition to turbulence resulted in higher $\partial u / \partial y$ over the second half of the plate at speeds of $117 \mathrm{~cm} / \mathrm{s}$. Fig. 6.10 demonstrates these effects. Coefficients of friction on the order of turbulent flat plate friction only occur at $117 \mathrm{~cm} / \mathrm{s}$ (black points on Fig. 6.10) and a plot of $C_{f x}$ for the blank inlet barrier (Fig. 6.10B) shows a later transition to friction of turbulent flat plate magnitudes. At the lower speeds (green points on Fig. 6.10), no such effect is visible. 

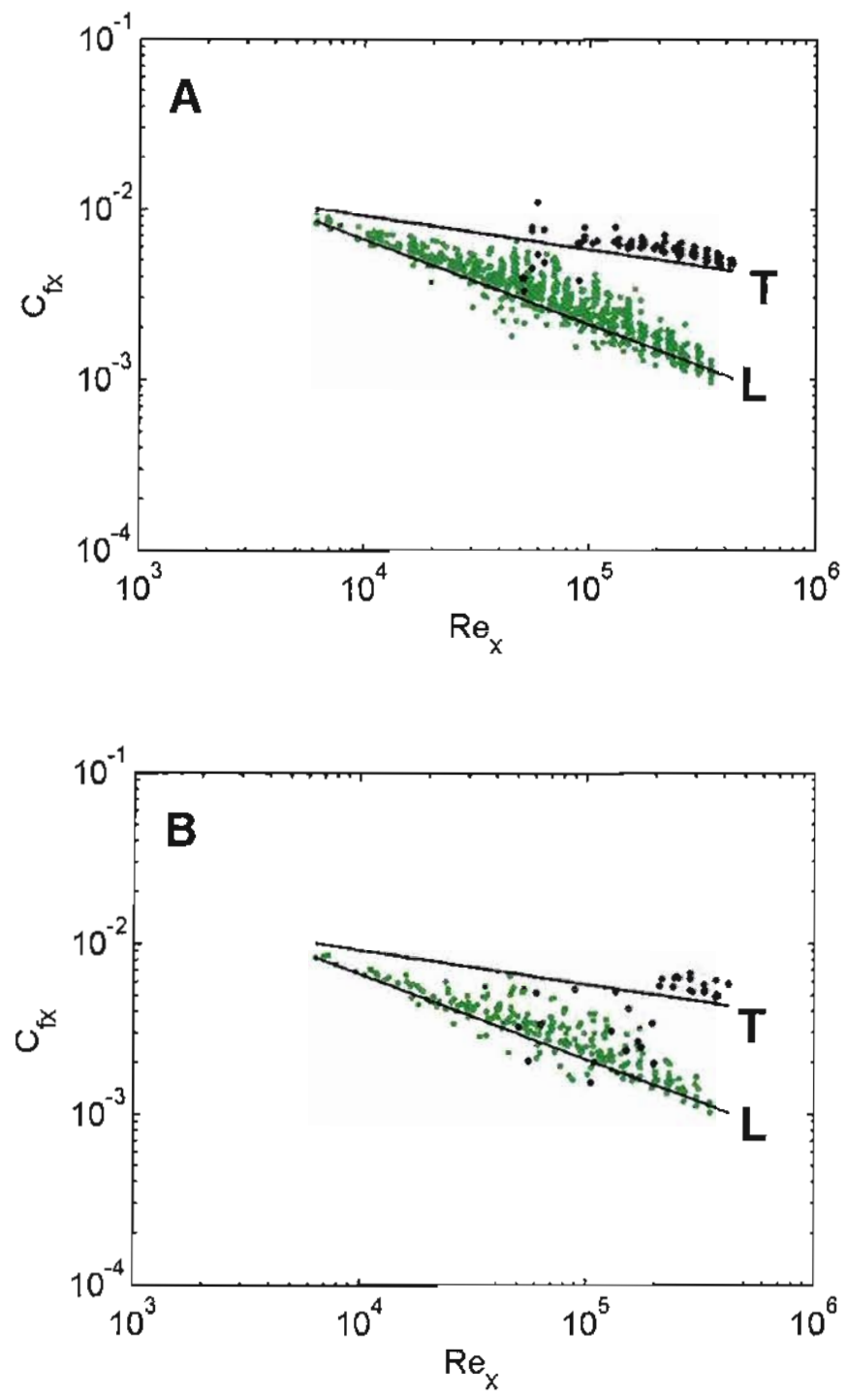

Fig. 6.10 Local coefficient of friction vs. length Reynolds number for all flat plate experiments. Black points correspond to $U=117 \mathrm{~cm} / \mathrm{s}$; green points correspond to all other flume speeds, $14.4-94.1 \mathrm{~cm} / \mathrm{s}$. ' $T$ ' marks the line for theoretical turbulent flat plate friction, and ' $\mathrm{L}$ ', laminar. (A) All experimental inlet barrier grids. (B) Blank inlet barrier. The plots illustrate the finding that the barrier grids only have a significant impact at 117 $\mathrm{cm} / \mathrm{s} . C_{f x}$ rises to a level similar to turbulent flat plate friction earlier when grid barriers are in place (A) signaling earlier transition to turbulence. 


\subsubsection{Relative local coefficient of friction, $C_{f x R}$, on the flat plate}

The relative local coefficient of friction was introduced in section 5.5 (Eq. 5.3) as a non-dimensional friction coefficient that could be plotted against relative position, $x / L$. It was proposed that this type of scaling should be used to properly compare local friction on fish. Although a flat plate should not require such scaling, it is none-the-less an instructive method of presenting the flat plate friction results. Fig. 6.11 shows $\mathrm{C}_{f x \mathrm{R}}$ vs. $x / L$ for three speeds $(14.4,94.1$ and $117 \mathrm{~cm} / \mathrm{s})$. The plot was produced from combined data from cases in which an inlet barrier grid was in place. Note how clearly the boundary layer behavior is communicated. The curve representing the lowest flume speed exhibits an increase in friction near the trailing edge. This corresponds with the boundary layer thinning observed in this region at lower speeds (e.g. Fig. 6.5). As speed increases to $94.1 \mathrm{~cm} / \mathrm{s}$ the trailing edge acceleration fades and the overall boundary layer converges to Blasius (i.e. $C_{f x R}=\sim 0$ ) as suggested by Fig. 6.6. Then, suddenly, at the next speed, $117 \mathrm{~cm} / \mathrm{s}$, the friction jumps to a magnitude on par with turbulent flat plate theory (i.e. $C_{f x R}=\sim 1$ ). Fig. 6.12 shows the case of $117 \mathrm{~cm} / \mathrm{s}$ again, but now in comparison to the data from the blank inlet barrier. The delayed transition to turbulent flow in the latter case is dramatically illustrated.

\subsection{Boundary layer flow over rigid fish}

The boundary layers over rigid fish were marked by separation of flow and transition to a turbulent boundary layer at speeds lower than observed in the flat plate. In general, local friction was greater than observed in the flat plate experiments and the boundary layer grew less regularly. 


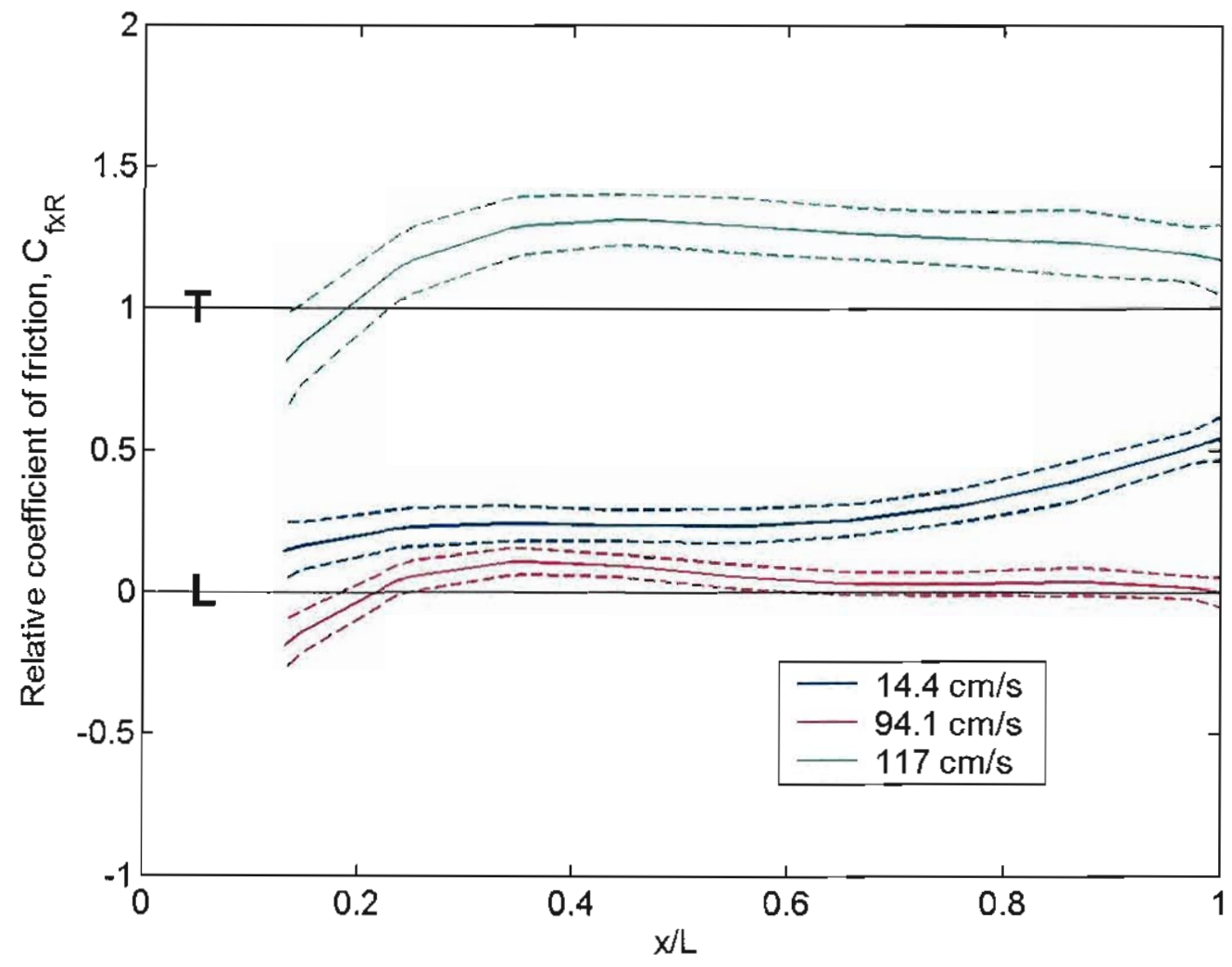

Fig. 6.11 Relative local coefficient of friction, $C_{\delta x R}$ vs. relative position, $x / L$, for flat plate experiments with experimental grids in place at $U=14.4,94.1$, and $117 \mathrm{~cm} / \mathrm{s}$. Dotted lines are $95 \%$ confidence limits. The graph illustrates the significant increase in local friction due to the presence of a turbulent boundary layer at $117 \mathrm{~cm} / \mathrm{s}$. The plot also shows the usefulness of $C_{f x: R}$, defined in this investigation, even for the flat plate. The rise in local friction near the trailing edge of the plate at lower flume speeds explains the spindle shaped distribution of data points on the plot of $C_{f x}$ vs. $R e_{x}$ in Fig. 6.10. The higher shear at the trailing edge at lower speeds agrees well with the thinner boundary layer observed in this region as shown in Fig. 6.5. 'T' marks the line for theoretical turbulent flat plate friction, and ' $L$ ', laminar. For clarity, flume speeds $21.8-68.7 \mathrm{~cm} / \mathrm{s}$ are not shown. They were found to be spread, in order, between the curves for 14.4 and $94.1 \mathrm{~cm} / \mathrm{s}$. 


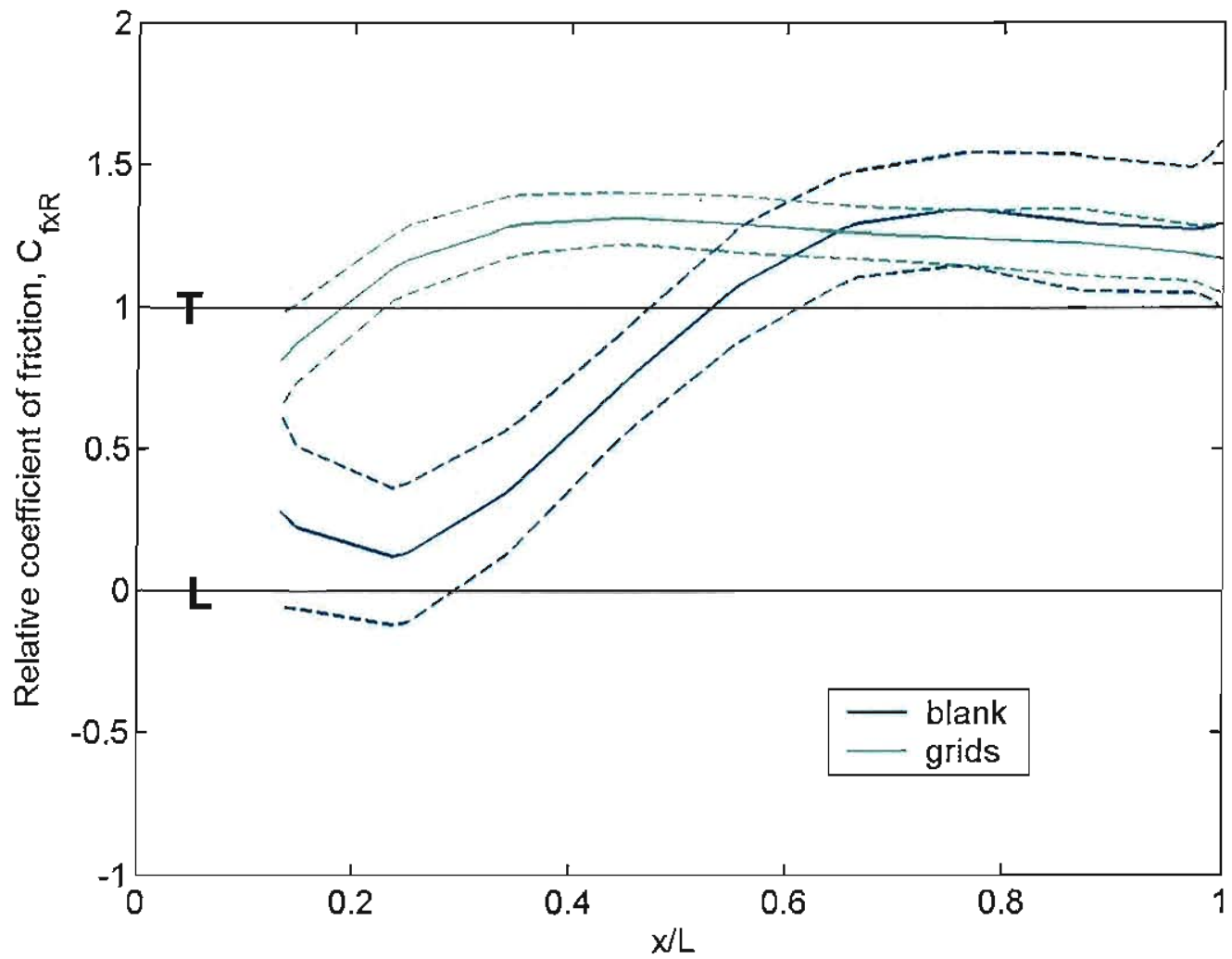

Fig. 6.12 Relative local coefficient of friction, $C_{f x R}$ vs. relative position, $x / L$, for flat plate experiments with experimental grids in place at $U=117 \mathrm{~cm} / \mathrm{s}$. Dotted lines are $95 \%$ confidence limits. The plot shows that transition occurs earlier with the experimental grids in place, as illustrated in Figs. 6.7 and 6.9. ' $T$ ' marks the line for theoretical turbulent flat plate friction, and ' $L$ ', laminar. 


\subsubsection{Boundary layer separation in rigid fish}

The boundary layer over a bluff body, such as a golf ball, typically separates shortly after the position of maximum cross-stream width, or 'thickness', due to the strong adverse (i.e. streamwise) pressure gradient resulting from the flow around the aft region of the object. The streamwise pressure gradient robs momentum from fluid in the boundary layer. Boundary layer profiles near the separation point exhibit an inflected shape as a result of deceleration near the wall. At some position, streamwise flow in the boundary layer actually reverses due to the pressure gradient. The meeting of the oppositely directed flows along the body surface requires that the flow be directed away from the body. This is the separation point. The velocity gradient at the body, and therefore the local wall shear stress, goes to zero. Flow beyond the separation point tends to be highly turbulent.

For more streamlined body shapes, such as an airfoil or fish, one expects separation to occur further aft since the adverse pressure gradient due to body shape less intense. In the fish observed here, separation occurred at different positions, with the earliest occurring in rigid scup around $x / L=0.65-0.75$ (Fig. 6.13). Separation in rigid mackerel occurred latest, near $x / L=0.85-0.95$. Bluefish and eel exhibited similar relative separation points near $x / L=0.7-0.8$. Notice the inflected $u$-profiles in Fig. 6.13 preceding separation in each case (mackerel at $x=26 \mathrm{~cm}$; bluefish at $x=26-29 \mathrm{~cm}$; scup at $x=15 \mathrm{~cm}$; eel at $x=32 \mathrm{~cm}$ ). In mackerel, bluefish and scup, outward flow (i.e. positive $y$-direction) is clearly exhibited in the v-profiles, as expected. The v-profile in the case of the eel is less definitive, as is the inflected profile, and in general, separation was less severe for the rigid eel. This is not surprising since eel exhibit the lowest thickness ratio ( $t / c$, i.e. maximum thickness/length) of the fish observed.

Separation results in a component of drag known as pressure drag. The pressure beyond the separation point is lower than it would be for ideal flow and results in a net 


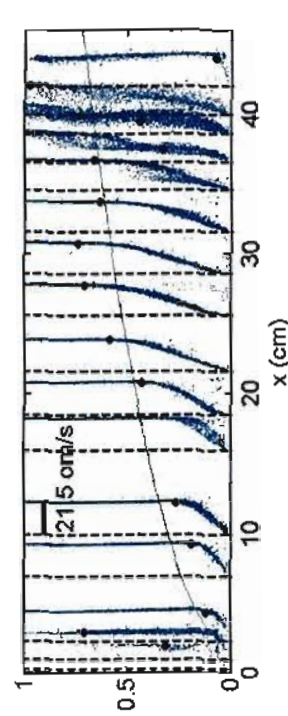

(u⿰) $A$

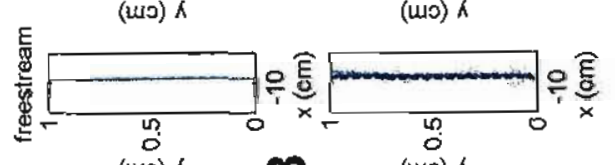

(uro) $\wedge$

$\infty$

(삐) $\Lambda$
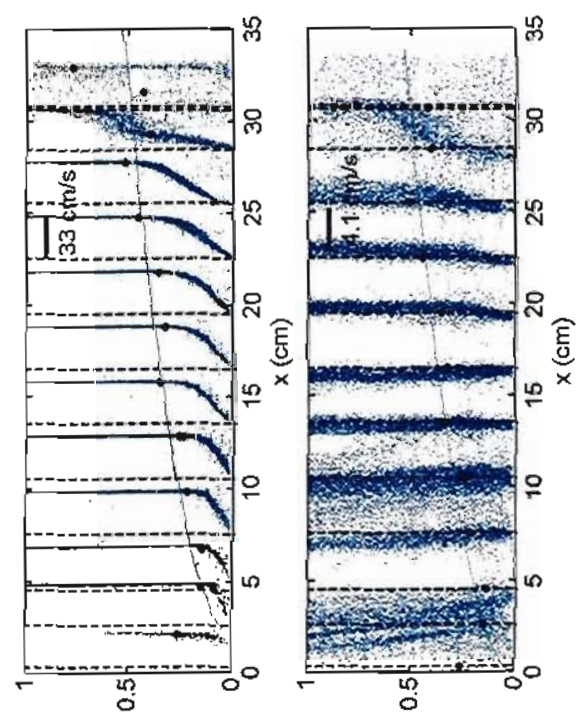

(wo) $\hbar$

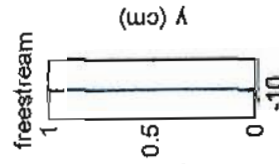

(wo) $\alpha$

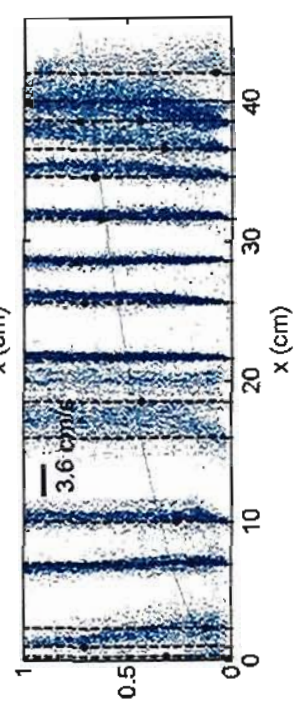

(u)

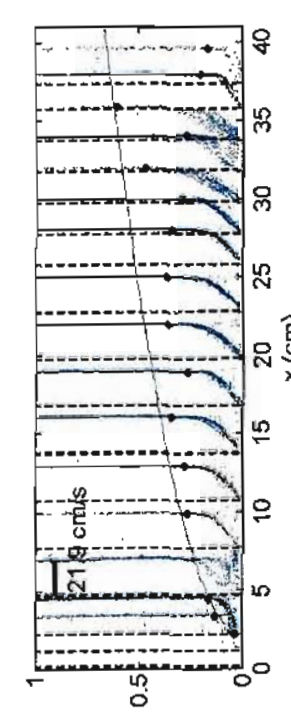

(us) $k$

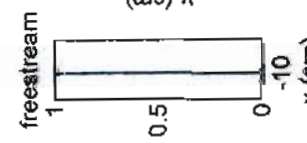

(wo) $\Lambda$

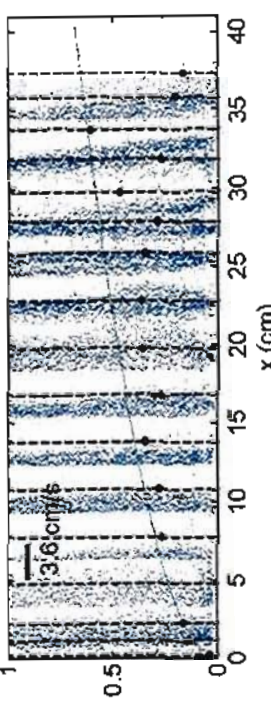

(us) $\wedge$

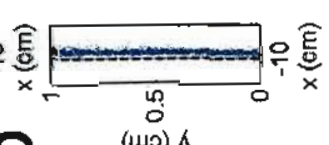

(40) $\alpha$
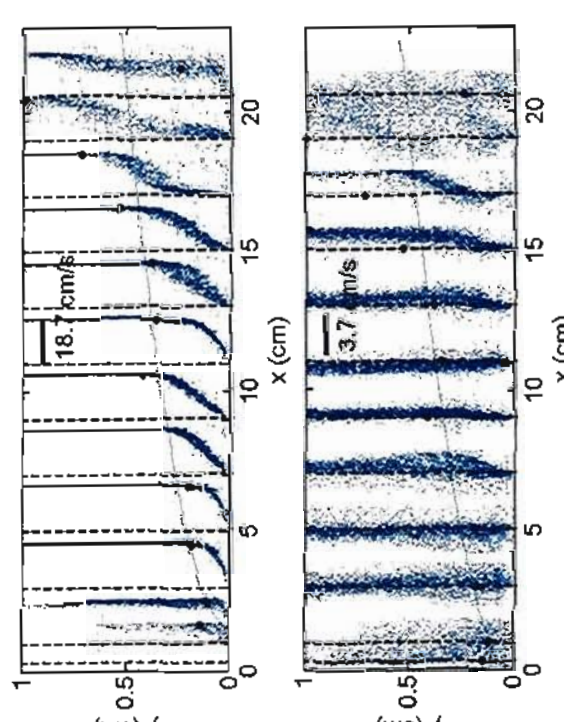

(w) $A$

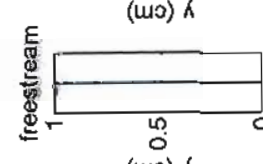

(w0) $k$

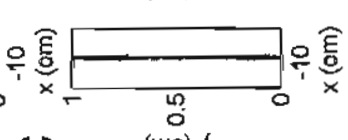

0 (w0) $\Lambda$

๑ 8

푤

응

定

जे

马

के 현

政

क के

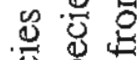

8

品至

息完

过

吕

$\div$

论

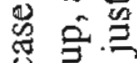

总

궁

$\circ \mathcal{O}$

용

픈

롱

농

足

品

행

实

클 임

焉志

8 눈

范密

응 光

.

要

텽 5

용

《

굥

불

我节 
rearward pressure force. Therefore, friction drag determined from the boundary layer is not the measure of the total drag. The presence of separation in all fish studied suggests that gliding could be costly for fish depending on the magnitude of the pressure drag. There was some evidence that when the boundary layer transitioned to turbulent that the separation point moved aft, but a detailed analysis will be left for future work. The same effect is observed in objects like golf balls. The presence of a turbulent boundary layer brings high momentum fluid closer to the wall, which allows the boundary layer to make more progress against the 'uphill' pressure gradient. Therefore the separation point moves downstream and pressure drag can be decreased significantly. The function of dimples on a golf ball is to trip the laminar boundary layer to turbulence for this effect.

\subsubsection{Early transition to a turbulent boundary layer}

In the f]at plate, transition to a turbulent boundary layer was not observed unti] $117 \mathrm{~cm} / \mathrm{s}$ at $R e_{x}$ around $1-3 \times 10^{5}$. Transition occurred earlier in rigid fish (Fig. 6.14), that is, at lower speeds or Reynolds numbers (mackerel, $U=69 \mathrm{~cm} / \mathrm{s}, R e_{x}=0.5-1.0 \mathrm{x}$ $10^{5}$; bluefish, $U=43 \mathrm{~cm} / \mathrm{s}, R e_{x}=0.4-0.9 \times 10^{5} ; \mathrm{scup}, U=38 \mathrm{~cm} / \mathrm{s}, R e_{x}=0.2-0.4 \mathrm{x}$ $10^{5}$; eel, $U=60 \mathrm{~cm} / \mathrm{s}, R e_{x}=0.5-1.0 \times 10^{5}$ ). Early transition is likely due to the flow destabilizing effects of surface structure near the front of the body associated with the mouth, eyes, and gills. In experimental fluid dynamics, a very thin wire on the surface of an object is often used to 'trip' the flow to turbulence. The fish forward structure is certainly large enough in scale to produce a similar effect. For a flat plate, empirical data shows that a single roughness element on the order of $1 / 6$ times $\delta_{99}$ is enough to decrease the critical $R e_{x}$ by the amount observed here (Schetz, 1993). Typical rigid fish boundary layer thickness near the leading edge was $<2.5 \mathrm{~mm}$. Therefore, roughness on the order of $0.4 \mathrm{~mm}$ (or less, closer to the leading edge) would be enough to trip the laminar boundary layer to turbulent. Distributed roughness can also encourage transition. Roughness with a 'roughness Reynolds number' ( $R e_{\kappa}=U \kappa / K=$ average roughness height) greater than 120 has been found to decrease the critical $R e_{x}$. This would correspond to roughness on 

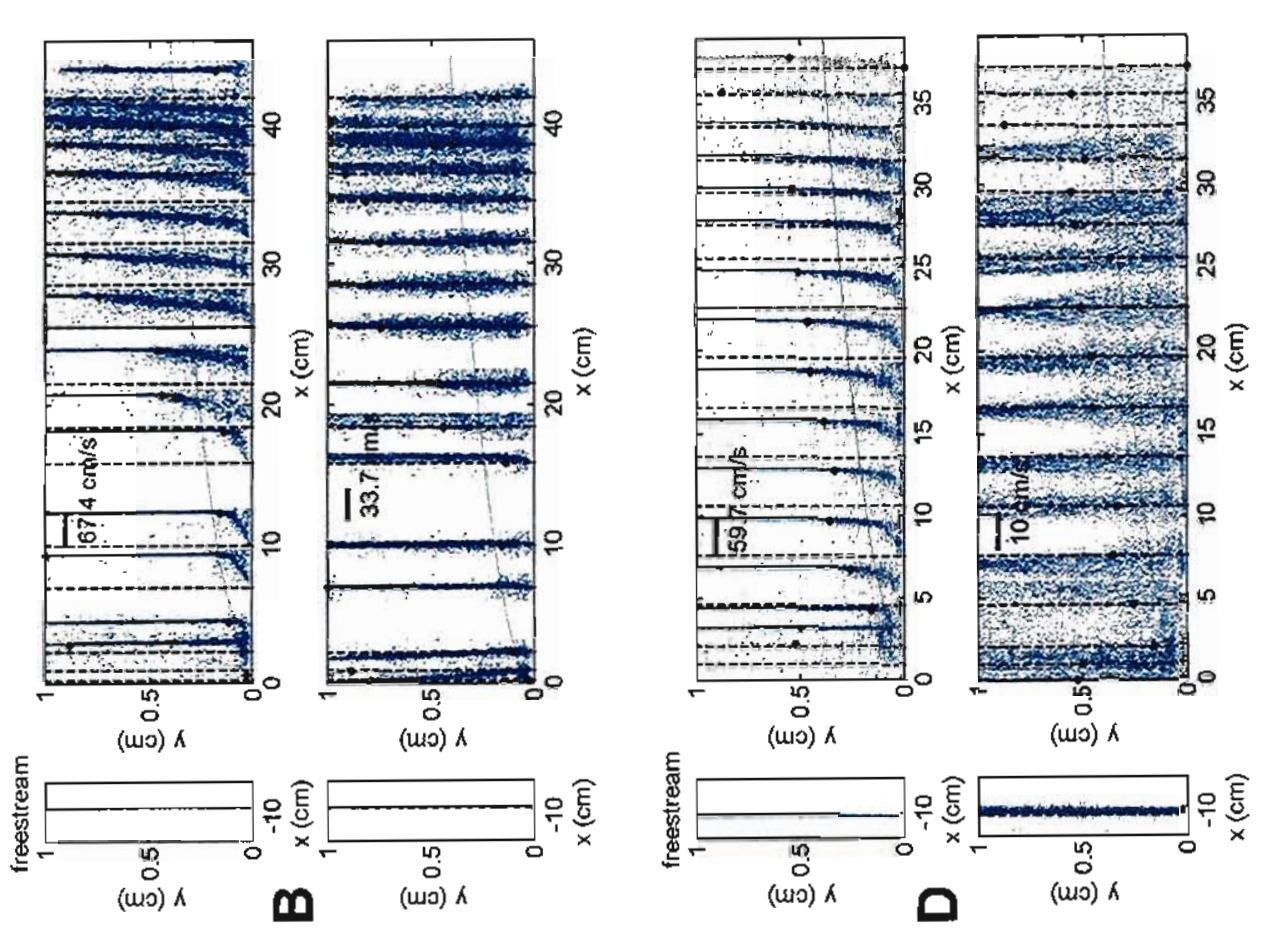

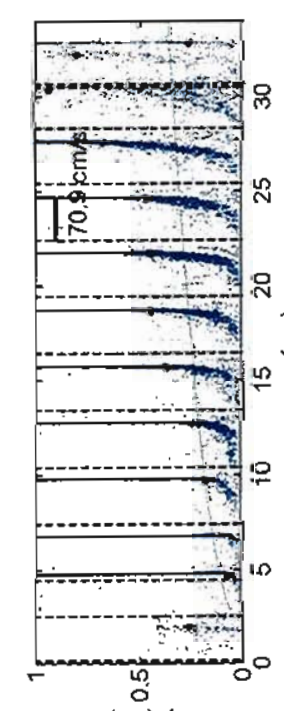

(us) $A$
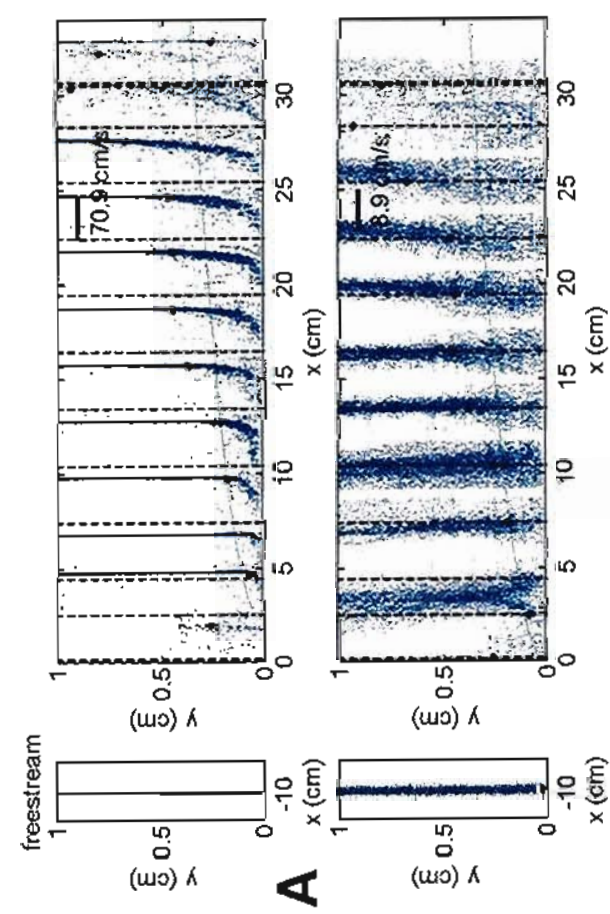

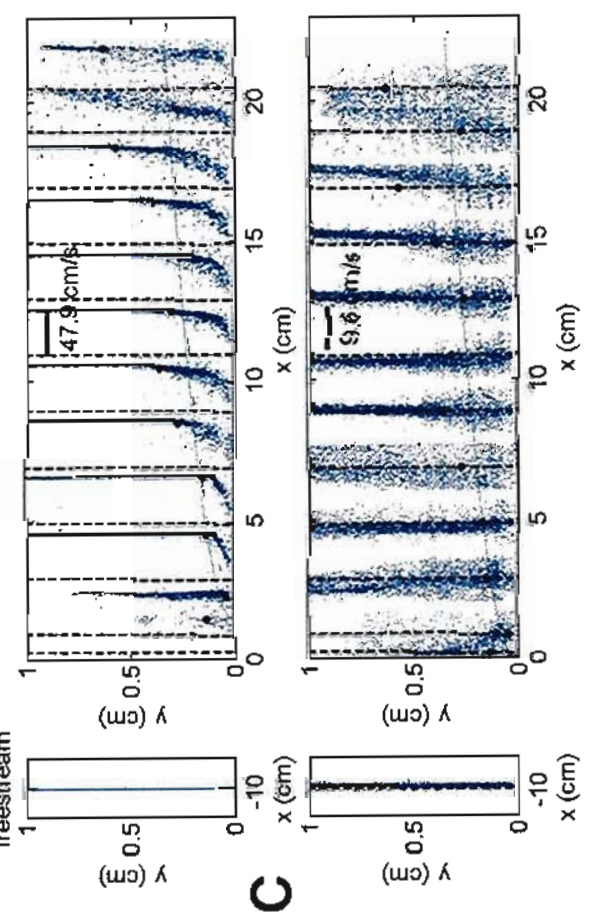

Page 184
要㤩三

용

\&

㤩怘

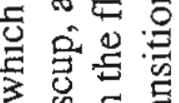

3 品

范

영 홍

造造

号寻品

는

可

舟

क ब过

ज氜

驱

若

过剀

屯屯

응을

능웅

岸范

宩哓

خे워

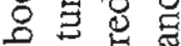

뭉용요

过

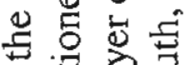

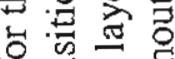

0 \%

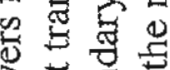

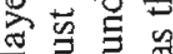

고용

응

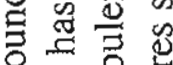

능을

प

5.

参资

解:

용형

的

寸通证

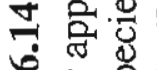

它总完 
the order of $0.2-0.3 \mathrm{~mm}$ with the fish observed here.

A study of surface roughness led to some interesting findings. Both the average roughness and the dominant wavelength of the roughness were determined. Mackerel, bluefish and eel all exhibited average roughness height between 0.02 and $0.05 \mathrm{~mm}$ over the majority of the body. Scup showed a larger range, namely 0.02 to $0.1 \mathrm{~mm}$. With these roughness heights, even at the highest swimming speeds observed, $R e_{k}$, would be less than 120. Nevertheless, some interesting aspects of roughness were observed. Mackerel in particular showed an extremely regular roughness wavelength of $0.8 \mathrm{~mm}$ for $x / L=0.4-0.95$. The roughness height was fairly constant at about $0.04 \mathrm{~mm}$ except for a consistent patch of roughness around $x / L=0.80-0.85$ where the roughness doubled. This still only translates to a $R e_{\kappa}$ of around $100-120$ for the highest speeds observed in mackerel, and at these speeds the flow was already turbulent upstream. Yet the position of this peak in roughness is very close to the position at which flow was observed to separate in the rigid fish and its function would be an interesting topic for further boundary layer work. A similar maximum, but not quite as sharply defined was observed in bluefish and scup. Roughness was $2-3$ times the average surrounding roughness. In all fish studied, roughness increased significantly at the head. Average roughness of greater than $0.1-0.2 \mathrm{~mm}$ was not uncommon. This strongly suggests that the turbulence observed was due to single element roughness at the head as discussed earlier. In fact, plots of boundary layer development that showed that transition usually occurred within $x / L=0-0.3$ (Fig. 6.14).

\subsubsection{Friction on a rigid fish}

The relative coefficient of friction, $C_{f x R}$, as a function of $x / L$ was determined for all rigid-fish cases. As for the flat plate (Fig. 6.11), speeds at which the flow was laminar exhibited similar $C_{f x R}$ values and so were grouped together. The same was true for the 'turbulent speeds' for each species and they were grouped, as well. Fig. 6.15 summarizes 

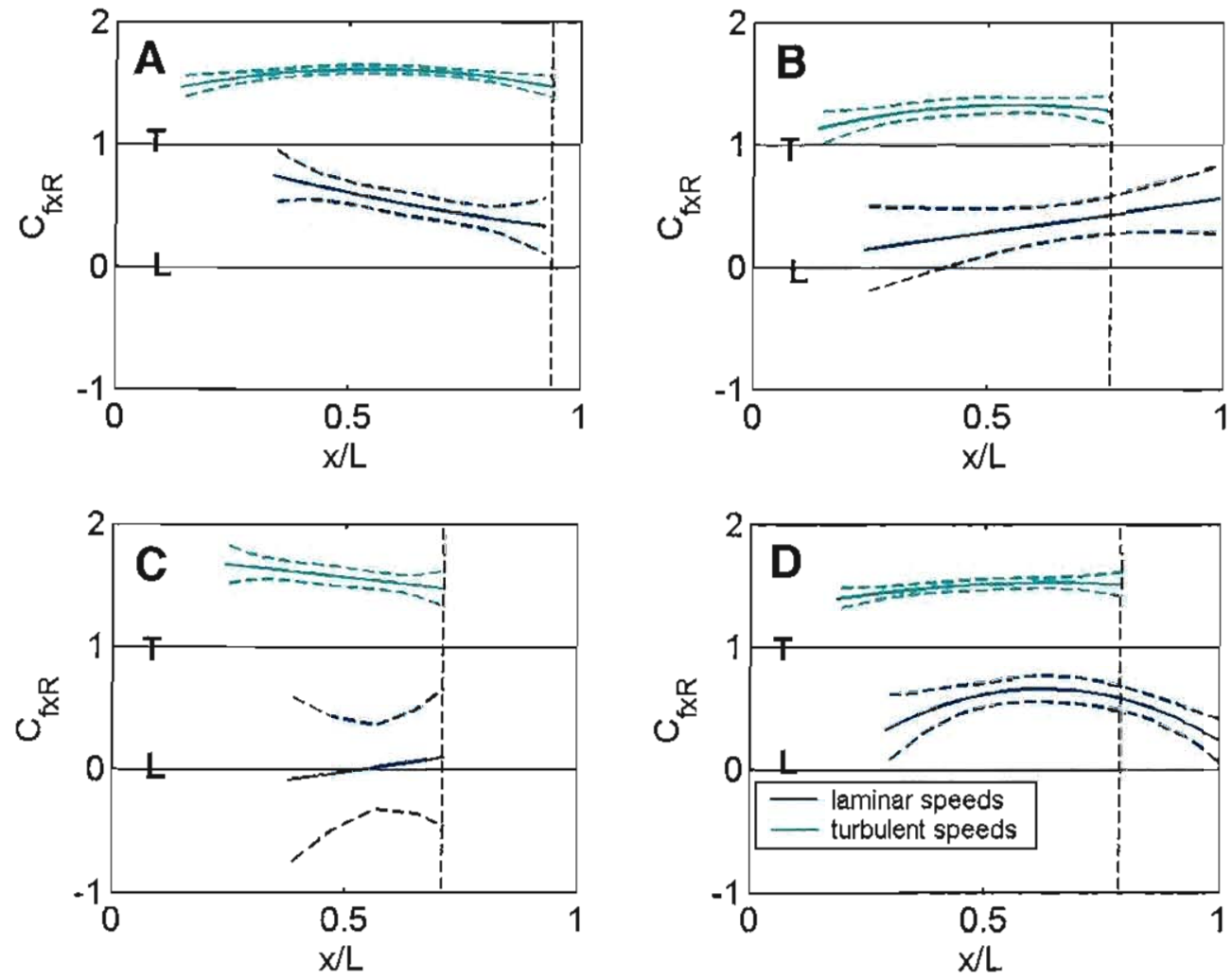

Fig. 6.15 A comparison of the relative coefficients of friction, $C_{f x R}$, with $95 \%$ confidence limits for the rigid-body cases of different fish species: (A) mackerel, (B) bluefish, (C) scup, and (D) eel. The black dotted line marks the approximate separation point. Data beyond the separation point is for low speed cases in bluefish and eel where flow did not appear to separate. The rigid fish cases in each species were divided into two groups depending on whether the boundary layer was laminar or turbulent. Compared to the flat plate (Fig. 6.11), the rigid fish tend to exhibit slightly higher local friction for both laminar and turbulent cases, except in the laminar cases of the scup. ' $T$ ' marks the line for theoretical turbulent flat plate friction, and ' $L$ ', laminar. 
the entire rigid body data set. When viewed in comparison to the flat plate it can be seen that the local friction on a rigid fish is, in general, slightly higher than on a flat plate. This is likely due to the fact that the forward region of the fish is sloped, which acts like a contraction, thinning the boundary layer and leading to higher wall shear stress. Of course, separation results in low friction drag near and aft of the separation point, but separation results in increased pressure drag. No dramatic differences are seen in the local friction between species, except that scup showed the lowest friction at laminar speeds than the other species. The uncertainties in the scup data however make the difference weakly significant. In the next chapter, the flat plate and rigid fish serve as 'standards' to which the live fish boundary layer are be compared. 

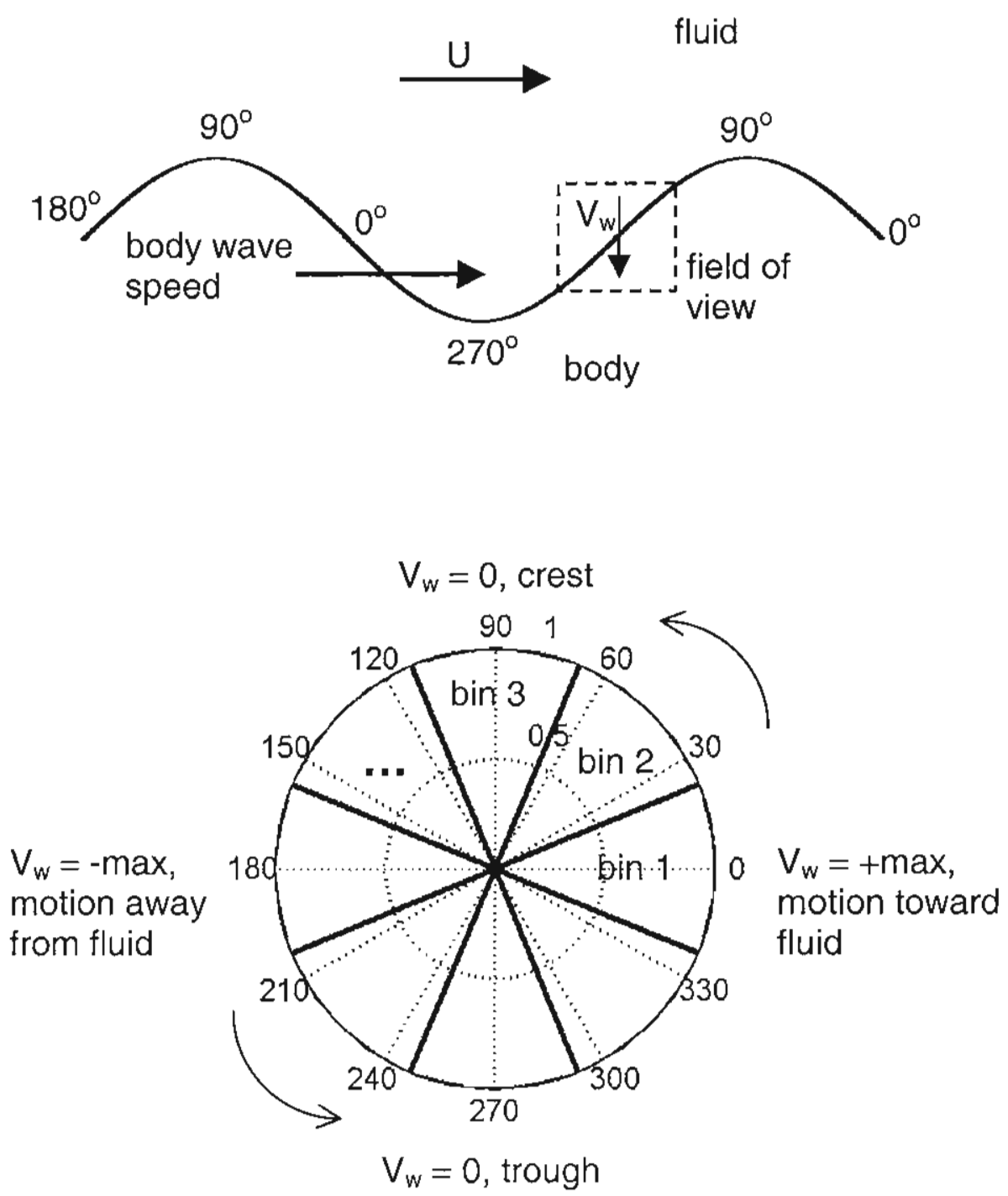

Fig. 7.1 Definition of body phase for transverse motion of the live fish and an illustrative description of phase binning for ensemble average boundary layer profiles. $v_{w}$ is the transverse velocity of the body. The progression of phase $\left(0^{\circ}-360^{\circ}\right)$ along the body surface is from right to left because the frame of reference is the 'stationary' field of view of the boundary layer profiling camera. All individual boundary layer profiles with a phase falling within the same bin and body segment (Fig. 4.1) were combined to produce ensemble average profiles. 


\section{Chapter 7}

\section{The boundary layer of swimming fish}

In this chapter, the findings of the live fish experiments are presented. Data from both individual boundary layer profiles (i.e. from individual image pairs) and ensemble average profiles is presented. Ensemble average profiles were constructed by binning individual boundary layer profile data from each of 10 equal segments along the body (Fig. 4.1) and at 8 sectors of phase of width $45^{\circ}$ (Fig. 7.1). Recall from the preliminary investigation that the crest of the body wave is defined as phase, $\phi$, equal to $90^{\circ}$, and trough, $\phi=270^{\circ}$. Therefore the transverse velocity of the body surface, $v_{w}$, into and away from the fluid is greatest at $\phi=0^{\circ}$ and $\phi=180^{\circ}$, respectively, on the side of the fish being profiled (Fig. 7.1). Before looking at the boundary layer, however, the body motions of the fish will be compared.

\subsection{Body wave amplitude and frequency}

In general, fish use a traveling body wave in locomotion that results in thrust producing interactions between the body surface and the surrounding flow. The motion of the surface may have a significant effect on the boundary layer and therefore it is important to compare the differences in motions across the species examined in this investigation. The species were handpicked to represent the range of wave-based, propulsive motions from various levels of the carangiform mode to the anguilliform mode as defined by Breder (1926). Breder's classification is based on the portion of the body apparently utilized most significantly in propulsion. Breder suggested that anguilliform swimmers (e.g. eel), which are characterized by elongated bodies of relatively constant height and lobate tails, produce thrust over a large portion of their body, whereas carangiform swimmers (i.e. mackerel, bluefish, and scup), with their well 
developed caudaI fins, produce the majority of thrust over the aft-most region of the body. Within the carangiform classification there are a variety of levels. For example, mackerel fall into a sub-group of carangiform called thunniform. Breder suggested that thunniform swimmers, characterized by lunate tails and significant necking at the peduncle, produce thrust exclusively with their tails. Scup and bluefish, however, have significantly less necking at the peduncle and even exhibit enhanced body height due to dorsal and ventral fins extending toward the peduncle. These features suggest that more of the body contributes to thrust production and places them between mackerel and eel in the classification of Breder.

Fig. 7.2 shows plots of body wave amplitude, $A$, vs. body position, $x$, at several swimming speeds, $U$, for all four species. All parameters were scaled by body length to facilitate comparison. This data comes from the nearfield camera view and is based on hundreds to thousands of data points for each plot. $95 \%$ confidence limits on the polynomial regressors plotted are not shown because they are not distinguishable from the curves themselves at the scale used. Standard deviation in relative amplitude (i.e. $A / L$ ) was generally about $10 \%$ of the local value. The plots at different speeds suggest that there is no clear trend in amplitude as a function of swimming speed for any of the species examined. Therefore, the data from all speeds were combined and plotted in Fig. 7.3 for the direct comparison of the four species.

The most interesting finding is, that although mackerel are categorized as thunniform, relative body wave amplitude was greater than any of the other fish over most of the fish length $(x / L=0.4-1.0)$. What more, eel exhibited the least relative body wave amplitude over the majority of fish length, except for the region $x / L=0.2-0.7$ in the case of scup. Therefore, although it is widely accepted that mackerel produce thrust almost exclusively with their caudal fin, this does not mean that there is less transverse motion along the body in comparison to anguilliform and other carangiform swimmers. 

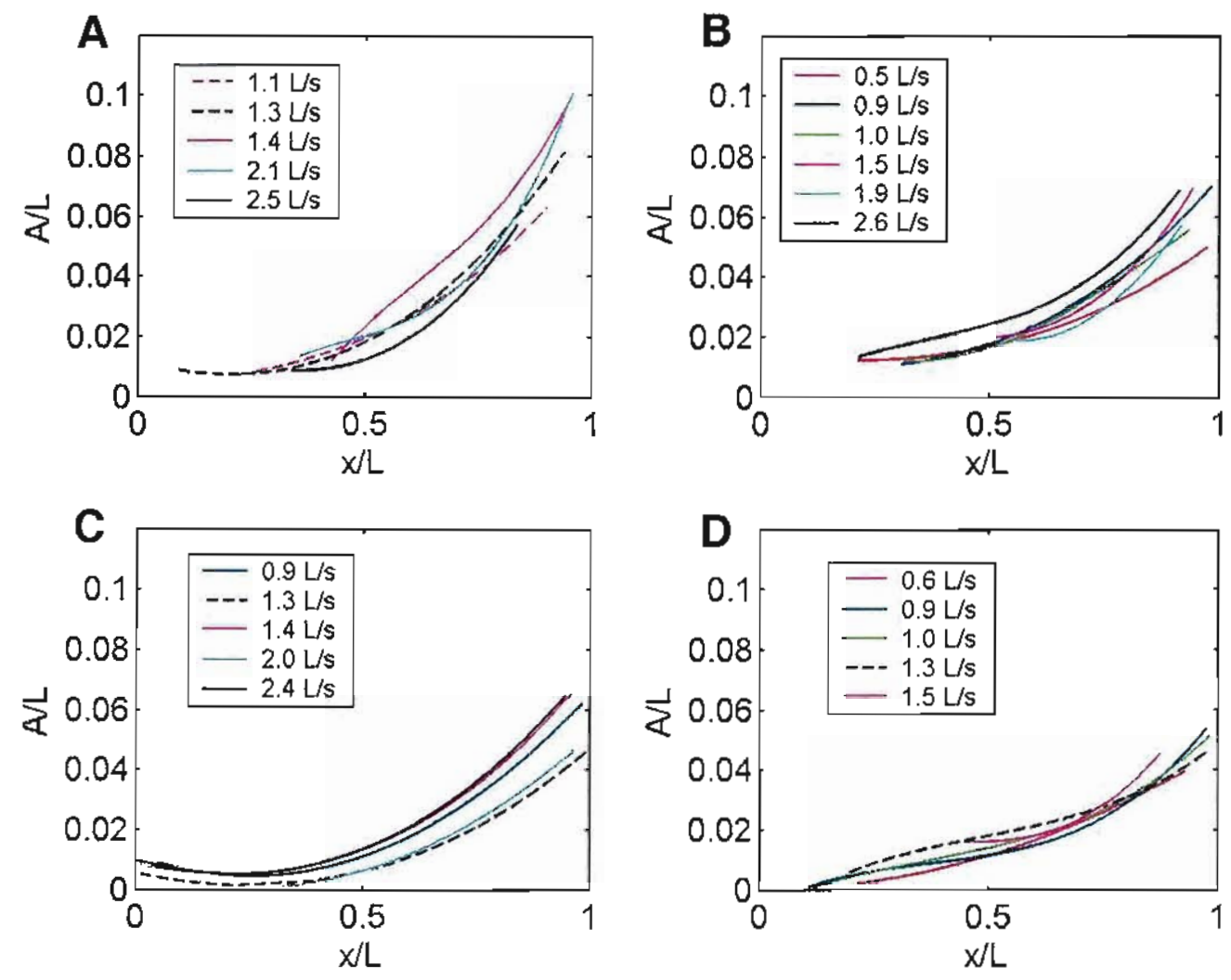

Fig. 7.2 Body wave amplitude as a function of body position and speed scaled by body length: (A) mackerel, (B) bluefish, (C) scup, and (D) eel. Plots of the same color on different graphs are of comparable speeds. In general, each curve represents a polynomial fit of hundreds to thousands of data points at each speed. Note that no obvious trend in amplitude with speed is apparent. 


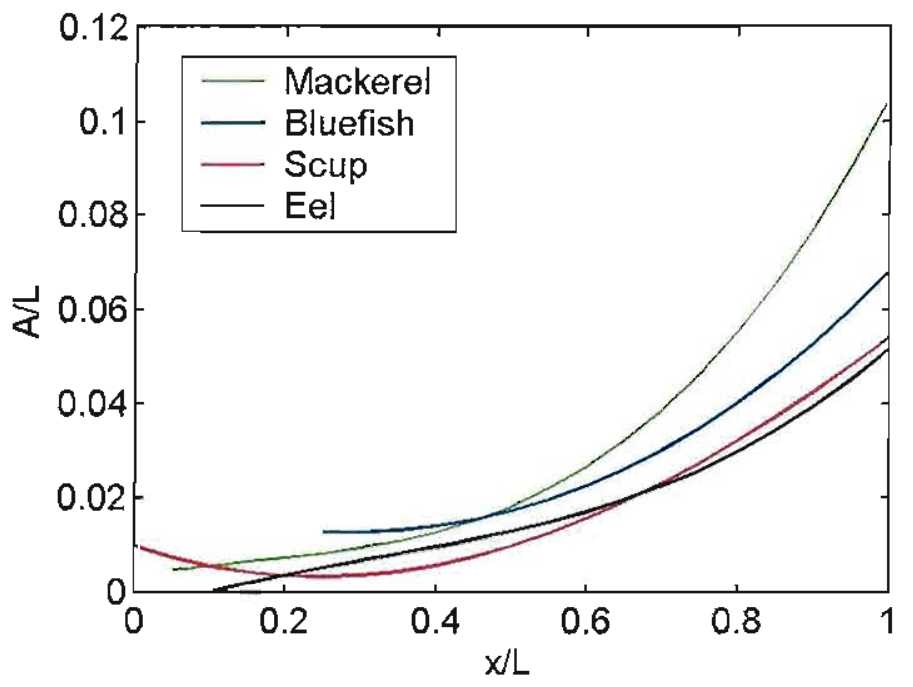

Fig. 7.3 Average body wave amplitude, $A$, scaled by body length, as a function of body position over the range of swimming speeds observed. Note that the thunniform mackerel exhibits the greatest degree of transverse body motion over more of the body except at the very front of the bluefish and scup. Eel, which defines the anguilliform swimming mode actually exhibits the lowest transverse body motion together with scup over its entire body. 
Nevertheless, a comparison of the growth in amplitude as a function of body position in these two species agrees with Breder's concepts of the locality of propulsion. The growth in amplitude is greatest in mackerel, which might be expected if it were chiefly concerned with moving its tail for propulsion. The eel exhibits a very gradual growth in amplitude along the body. If a larger region of the body is being used for propulsion, it may follow that the contribution of each sub-segment is equally as effective for thrust at a given local amplitude and that dramatic growth in amplitude is unnecessary, and perhaps even less efficient. Growth rates in bluefish and scup were nearly the same and were in between mackerel and eel_ not surprising if they utilize a combination of body and tail thrust.

Fig. 7.3 also shows that transverse motions in the mid- to forward-body region are greatest in bluefish, followed by mackerel, eel and then scup with the least. The scaled sideview images of the fish in Fig. 4.1 show that scup clearly have the 'tallest' body shape. This explains the resistance to transverse motion in the forward region. The large surface area of the forward body produces a larger counter torque to that produces by the motion of the tail. Lighthill (1970) points out that this reduction in transverse motion of the forward body contributes to hydrodynamic efficiency. Bluefish have a very large surface area tail and aft propulsive region $(x / L=0.6-1.0)$ relative to its forward body in comparison to all three other species, and therefore the high degree of transverse motion in the forward body is not surprising. At the head, body amplitude in scup actually increases above eel and mackerel (Fig. 7.3). In fact, amplitude goes to zero in the eel. The stability of the forward region of the eel can be understood on the basis of the gradual growth in body wave amplitude and the relatively long region over which the body oscillates. The extent of the region is on the order of a body wavelength and the torque producing forces are more balanced.

Fig. 7.4A shows absolute transverse body velocity, $v_{w}$, at the trailing edge vs. swimming speed for the fish studied. In general, eel and mackerel exhibit higher $v_{w}$ than 

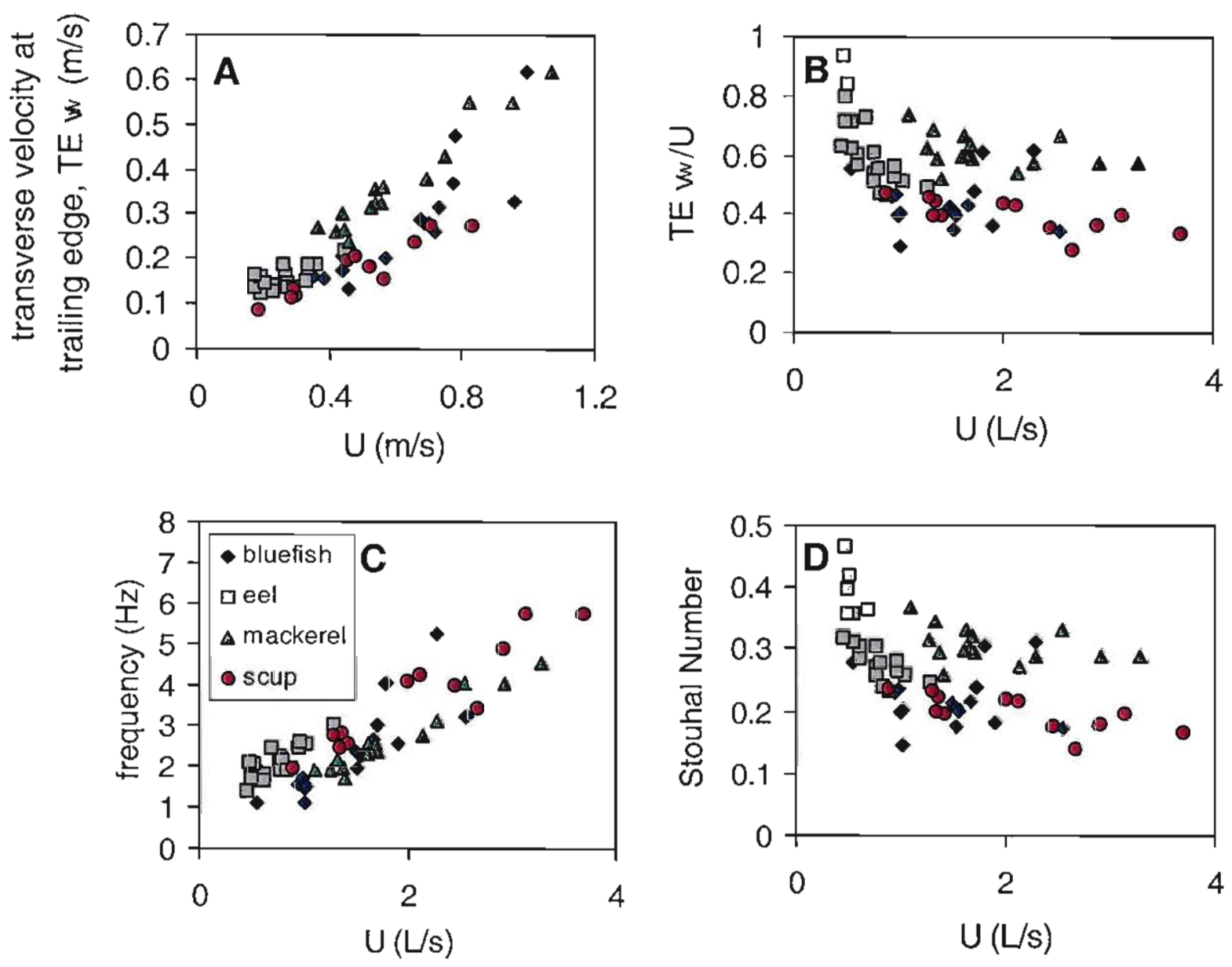

Fig. 7.4 (A) Transverse velocity, $v_{w}$, at the trailing edge, $(B)$ transverse velocity, $v_{w}$, at the trailing edge scaled by $U$, (C) frequency, $f$, and (D) Strouhal number, $2 A f / U$, in live swimming fish. 
scup and bluefish at the same absolute swimming speeds. This is more obvious when $v_{w}$ is scaled by swimming speed and plotted against swimming speed in body lengths per second (Fig. 7.4B). Eel exhibit the highest relative transverse body velocities at the trailing edge, $\mathrm{v}_{\mathrm{w}} / U=0.5-0.95$, but only at low speeds. At higher speeds, mackerel exhibit the highest relative transverse velocities- -0.5 to 0.7 . In bluefish, the values ranged from 0.3 to 0.6 , and in scup, 0.3 to 0.5 . In general, the quantity appears to decrease with increasing swimming speed and then level off at higher speeds. The value must level off eventually, since the relative transverse body velocity cannot go to zero in steady swimming. Transverse velocity is likely an important factor in fish boundary layer development since the back and forth motion of the body cross-section alone would result in an oscillatory boundary layer. If a cylinder were oscillated perpendicular to its long axis, one would expect a boundary layer flow alternately approaching that of the fore and aft faces of a cylinder in a steady flow. Of course, the boundary layer would be in a constant state of flux and development, especially at high frequencies and small body amplitude. Nevertheless, one would expect the boundary layer to be thin on the face of the cylinder driving into the surrounding fluid, and then to separate rapidly after changing directions.

Fig. 7.4C shows body wave frequency as a function of swimming speed in body lengths per second. Scup and eel tend to exhibit a slightly higher frequency than bluefish and mackerel at the same scaled swimming speeds. Since scup were on average 0.5 to 0.7 times the length of the other species, a plot of frequency vs. absolute swimming speed would reveal that scup require a body wave frequency of about 2 times that in the other species. This is similar to land based locomotion in the sense that animals with smaller bodies tend to have smaller legs and therefore need to take more steps to cover the same ground, and therefore require a higher frequency of leg motion to cover the distance in the same time. Of the other three species, the bluefish is most similar to the scup in body and caudal fin shape. In both fish, the ratio of caudal fin height to body length was about 0.3. Therefore, since scup were about 0.5 times the length of bluefish, the caudal fin was 
only half the size of that in bluefish. The scup must beat this smaller tail twice as fast to keep up with a bluefish. Of the three carangiform swimmers, the scup would certainly be considered least known for its swimming prowess. During specimen collection, mackerel and bluefish were generally found cruising in open and/or fast moving water, whereas scup were generally found 'camped-out' near the bottom and near structure.

A dimensionless frequency known as Strouhal number, $S t$, is plotted in Fig. 7.4D. Triantafyllou et al. $(1991,1993)$ showed that in nearly all fish utilizing undulatory propulsion Strouhal number tends to fall between 0.25 and 0.40 . They calculated Stouhal number using $S t=A f J$, where $A$ is 2 times the body wave amplitude at the trailing edge, $f$ is the body wave frequency, and $U$ the swimming speed. Note that the variable $A$ used in this thesis refers to body wave amplitude. Stouhal number defined this way is the same as $0.5 \mathrm{v}_{\mathrm{w}} / U$, i.e. one half times the values plotted in Fig. 7.4B (one half of the transverse body velocity at the trailing edge scaled by swimming speed). Strouhal numbers ranged from 0.15 to almost 0.5 , but in the majority of cases it fell between 0.15 and 0.35 , with averages of 0.2 in scup, 0.2 in bluefish, 0.3 in eel, and 0.3 in mackerel.

\subsection{Bluefish boundary layer}

Fig. 7.5 shows ensemble boundary layer profiles in a swimming bluefish at four phase bins $\left(45^{\circ}, 135^{\circ}, 225^{\circ}, 315^{\circ}\right)$ representing the full cycle of body motion. A slow swimming speed $(24 \mathrm{~cm} / \mathrm{s}, 0.55 \mathrm{~L} / \mathrm{s})$ is shown since the effects of body motion on the boundary layer are more easily demonstrated for laminar flow. As in the rigid fish and the flat plate, the vertical dashed lines represent the $y$-axis of the adjacent profile. In the $u$-profiles the adjacent profile is to the right, or streamwise, and in the v-profile, the axes tend to overlay the data. Little to no data was acquired for $x / L=0-0.3$, which represents the head and operculum in bluefish (Fig. 4.1). The same was true for the region $x / L=0.9$ -1.0 , which is on the caudal fin. Scatter in the data is due to the fact that (1) profiles are not always identical at the same phase and body position, and (2) a moving body surface 


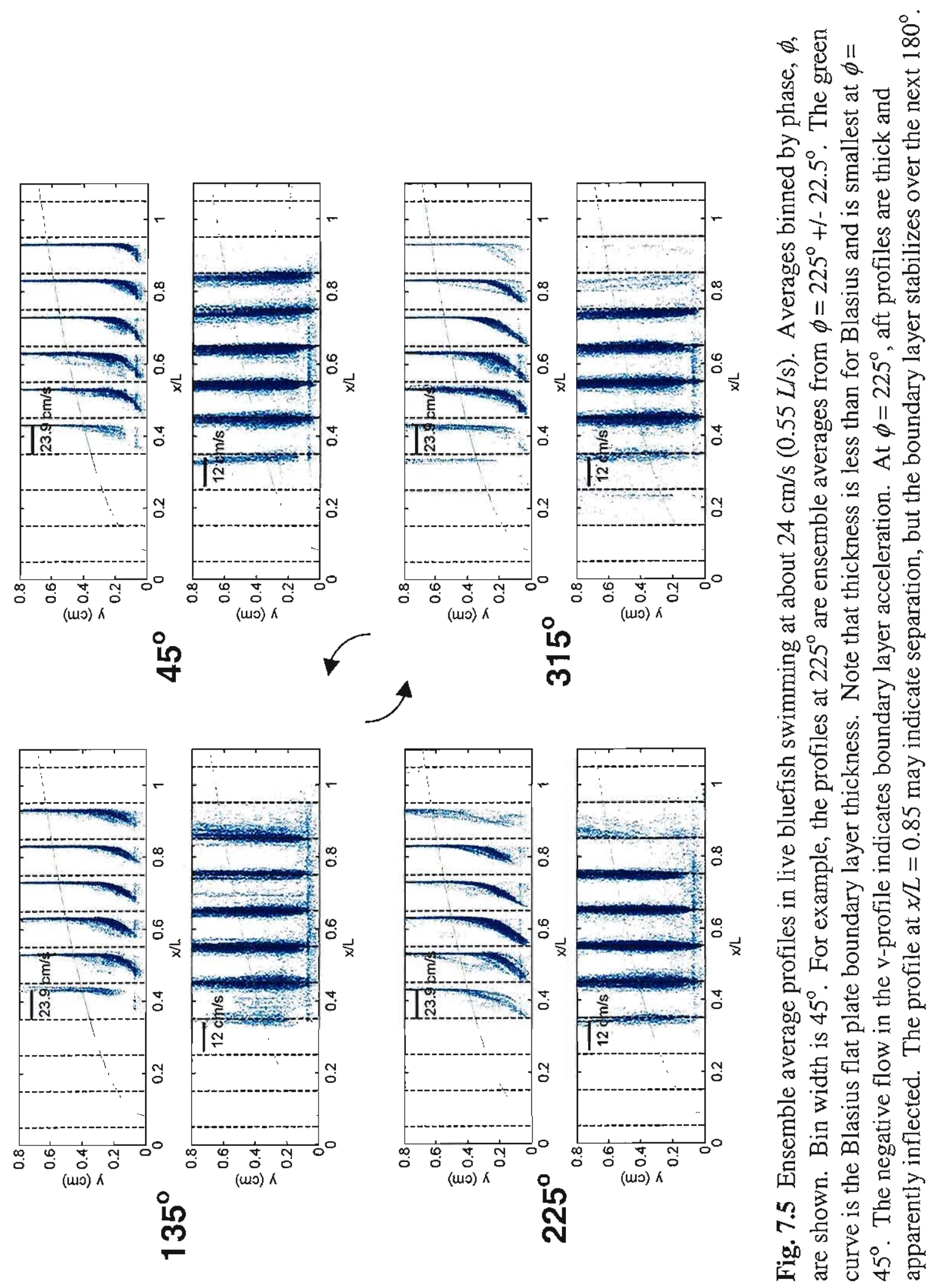


pushes the particle tracking code to the edge of its functionality. These issues were expected and were the main reasons for constructing ensemble profiles in the first place. The average profile shapes at the given positions and phases are revealed by the dark, high data density regions on the profile plots. Bin size also affects the sharpness of the ensemble profiles. Large bin sizes smear the profiles in the $y$-direction due to differences in boundary layer thickness in profiles within a given bin. Nevertheless, ten bins of streamwise position, and 8 bins of phase, were found to produce ensemble profiles with coherent profile shape. Note that each of the 4 sets of $u$-and v-profiles in Fig. 7.5 are not instantaneous plots of the entire boundary layer along the fish, rather they show the instantaneous boundary layer profiles at each body segment at a particular phase. It is the boundary layer as would be observed by an observer moving with the body wave at a given phase.

\subsubsection{Bluefish boundary layer thickness}

The region of the boundary layer that was resolved $(x / L=0.3-0.9)$ shows two sub-regions of differing boundary layer thickness $-x / L=0.3-0.6$, and $x / L=0.6-0.9$. The more forward region exhibits a greater thickness than the aft region at $\phi=45^{\circ}$. In general, the thickness at this phase for $x / L=0.3-0.6$ tends to exceed that of a Blasius boundary layer. The profiles in this region also exhibit smearing suggestive of fluctuations in the local profiles. By contrast the boundary layer of the aft sub-region at $\phi$ $=45^{\circ}$ is significantly thinner than a Blasius boundary layer and generally less smeared than the forward region. The bluefish sideview image of Fig. 4.1 shows that outlet of the operculum is at $x / L=0.3$ and that the pectoral fin spans $x / L=0.3-0.44$. Both of these could explain the thickness of the boundary layer in the forward sub-region. Outflow from the operculum and the motion of the pectoral fin and operculum could result in a thicker boundary layer. These effects appear to be significantly damped out over the aft sub-region as exhibited by the thin laminar boundary layer profiles, especially at $\phi=45^{\circ}$, but also at $135^{\circ}$ and $315^{\circ}$. 
At $\phi=225^{\circ}$, the boundary layer is a bit more complicated. 'The profile with its axis at $x / L=0.35$ is thicker than Blasius, but the next profile $(x / L=0.45)$ and all subsequent profiles until $x / L=0.85$ are thinner than Blasius. The boundary layer at $x / L=$ 0.85 is coherent, but thick and quite scattered. This is similar to what was seen in the rigid fish when flow separated in the aft region of the body (see Fig. 6.13B). In fact, the boundary layers are increasingly inflected as one moves from $x / L=0.55$ to 0.75 at this phase $\left(225^{\circ}\right)$. This was also observed in the rigid fish upstream of the separation point. Basically, all the evidence suggests that flow separates or comes very close to separating close to $x / L=0.85$ at $\phi=225^{\circ}$, i.e. as the body surface is retreating from the fluid on the side of the fish being profiled. Recall that in the discussion of body oscillation, it was explained that transverse motion away from the fluid might be expected to initiate boundary layer separation. Increased scatter in the profile at this position and phase suggests that the particle tracking code is beginning to having difficulty resolving the flow, which means it may be increasingly turbulent, as was observed in the flow near and beyond the separation point in rigid fish.

At $\phi=45^{\circ}$, however, the boundary layer profile at $x / L=0.85$ is very thin, highly coherent and laminar-like in shape. Therefore, the boundary layer at this position is fluctuating between a thick, inflected, low shear--and perhaps even separated-profile to a fully attached, thin, laminar, high shear profile. The v-profiles support this, showing that at $\phi=225^{\circ}$ there is significant outflow $(+v)$ in the boundary layer, which is expected in a highly decelerating boundary layer as is the case for incipient separation. At $\phi=45^{\circ}$, the opposite is true. There is significant inward flow $(-v)$, which is expected in an accelerating boundary layer. An accelerating boundary layer generally thins and is highly stable, i.e. remains attached for greater streamwise distances. Looking at Fig. 7.5 as a whole, one realizes that this oscillation is present over the entire boundary layer, but more prominent as one moves toward the aft region of the body, where the body wave amplitude it greatest. All of the v-profiles lean to the negative at $\phi=45^{\circ}$, and to some 


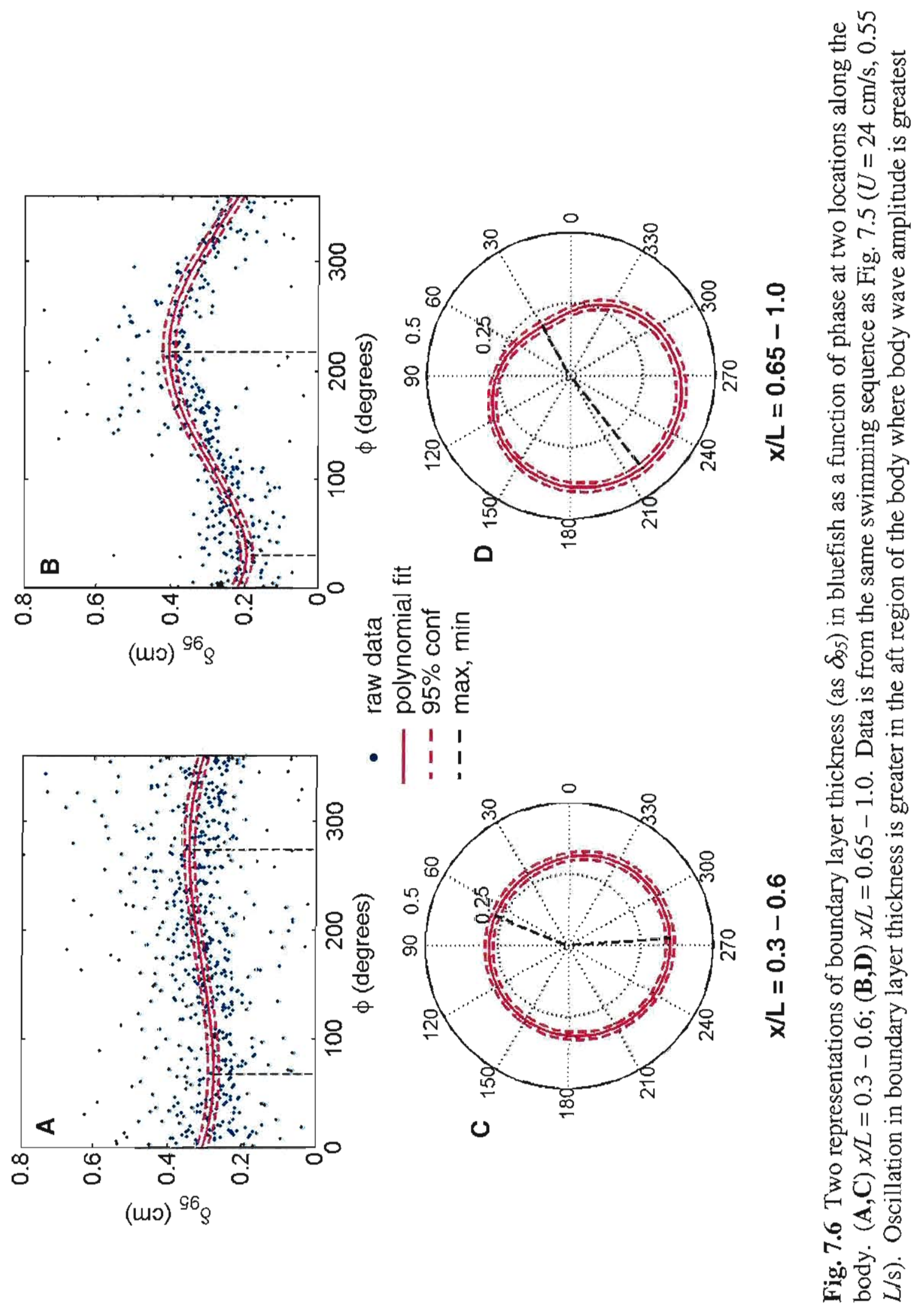

Page 200 
degree at $\phi=315^{\circ}$. Boundary layer thickness tends to be least at $\phi=45^{\circ}$ overall. It then increases at $135^{\circ}$, then more at $225^{\circ}$ with the exception of the more forward positions. It then begins to thin at $\phi=315^{\circ}$. Fig. 7.6 presents a closer look at boundary layer thickness for the profiles of Fig. 7.5. In this case, $\delta_{95}$ was used. Figs. 7.6A and 7.6B show thickness data as a function of phase from the individual profiles that make up the ensemble profiles over the two sub-regions defined earlier. Each data point represents the profile determined from a single image pair. A fourth degree polynomial constrained to be periodic (i.e. $y\left(0^{\circ}\right)=y\left(360^{\circ}\right)$ and $y^{\prime}\left(0^{\circ}\right)=y^{\prime}\left(360^{\circ}\right)$ ), was fit to the data by linear regression and is shown on the plot with $95 \%$ confidence limits in the mean. For a fluctuation in the mean to be significant the $95 \%$ confidence limits must not overlap. This is barely the case for the maximum and minimum thickness indicated on Fig. 7.6A by black, vertical, dashed lines, but the fluctuation is nevertheless significant. The fluctuation in the aft sub-region is much more pronounced and clearly significant (Fig. 7.6B). Note that the position of maximum boundary layer thickness over the aft subregion occurs at around $220^{\circ}$ and the minimum at $\phi=30^{\circ}$ (Fig. 7.6B), close to the centers of the phase bins exhibiting extremes in thickness in Fig. $7.5\left(\phi=225^{\circ}\right.$ and $45^{\circ}$, respectively). Figs. $7.6 \mathrm{C}$ and $7.6 \mathrm{D}$ are polar plots of the same regressor, confidence limits and maximum and minimum values as in Figs. 7.6A and 7.6 B, respectively. The scale for $\delta_{95}$ is labeled between $\phi=60^{\circ}$ and $90^{\circ}$. The data points are left out for clarity. Polar plots will be used for $\delta_{95}$ comparisons throughout the rest of this investigation.

\subsubsection{Bluefish boundary layer local coefficient of friction}

Fig. 7.7 is similar to Fig. 7.6 except the parameter plotted is the local friction coefficient $C_{f x}$. Notice that local friction oscillates essentially $180^{\circ}$ out of phase with boundary layer thickness, as would be expected. Thin boundary layers are generally high shear boundary layers. Thick, inflected boundary layers, like those observed at $\phi=225^{\circ}$ (Fig. 7.5) exhibit low shear. Fig. 7.7D shows that the lowest shear occurs at about $\phi=$ $240^{\circ}$. The average local friction over the entire locomotive cycle was determined to be 


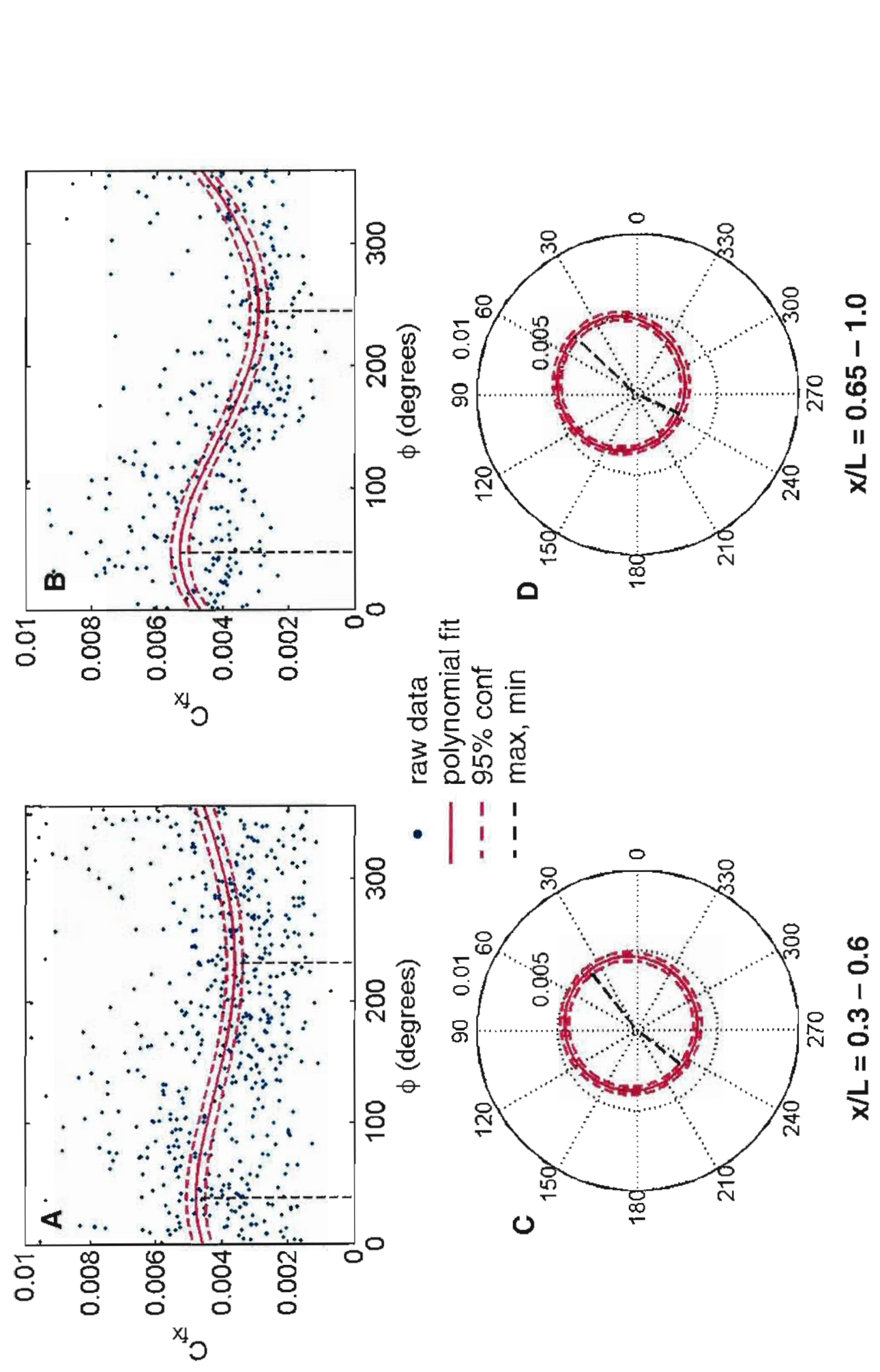

ำ

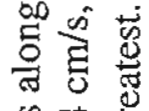

范

. 11.2

马용

0 늘

हो

西证

品

范

范焉

Б 요

总总

$\pi$

용

可 0

焉莺吉

0

토용용

स

$\circlearrowleft$

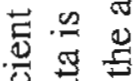

焉急

80.

둥

苟

政

敢 11 :

등

पै

동

兽

包

范

造

30

$r \leqslant \&$

음 
similar to that of the rigid bluefish. This will be examined in detail at the end of this chapter.

\subsubsection{Significance of bluefish boundary layer findings}

These findings lead to three very significant remarks concerning fish boundary layers. First, the boundary layer over a swimming bluefish, except for the suggestion of momentary separation or incipient separation at $\phi=225^{\circ}$ and the determination of similar time-averaged friction, is quite different from the boundary layer over the rigid fish. Boundary layer thickness and local friction fluctuate at the same frequency as the body wave and separation appears to be suppressed over the majority of the swimming cycle. Of course, separation must occur at some position near the trailing edge, but it occurs downstream of where it occurs on the rigid fish. In this way, the motion of the body of the fish appears to be stabilizing the boundary layer, possibly by the mechanism observed in the waving plate of Taneda and Tomonari (1974). The delay of separation due to the fish motion would be expected to reduce the pressure drag somewhat on a swimming fish compared to the rigid fish.

Second, the motion of the body of the fish, although it clearly affects the boundary layer and instantaneous local friction, does not appear to have an affect on average local skin friction. The significance of this will be discussed in more detail at the end of the chapter.

Finally, the findings regarding the fluctuation of thickness and local friction in the fish boundary layer are very similar to what was observed in the preliminary investigation (Figs. 3.6, 3.8, 3.9, 3.10, 3.12). The same oscillation of boundary layer thickness (Figs. 3.6, 3.8, 3.12) and the v-profile (Figs. 3.9, 3.10) were observed in scup and smooth dogfish. In addition, the effects were observed to increase as one moved aft along the body (Fig. 3.8, 3.9). It is very significant that two independent analyses of the 
boundary layers of swimming fish--one manual and one automated--have shown such excellent correspondence. Nevertheless, it was the case that friction drag was found to be higher in a swimming dogfish than in a 'rigid' dogfish, and that procession of the phase of extremes in thickness and local friction were observed, and neither of these was observed in bluefish. But the dogfish body motion and propulsive mode (anguilliform) is quite different from that of bluefish, and therefore these differences may not be comparable. In the preliminary investigation, scup were observed to exhibit very little fluctuation in local friction and boundary layer thickness (Fig. 3.6). In addition, swimming scup showed less evidence of enhanced friction due to body motion than that in a swimming dogfish. Bluefish are certainly more like scup than dogfish in body shape and swimming mode.

\subsection{The scup boundary layer}

Fig. 7.8 shows an example of the boundary layer over a swimming scup. A relatively fast swimming speed is shown $(U=61 \mathrm{~cm} / \mathrm{s}, 2.86 \mathrm{~L} / \mathrm{s})$ and transition of the boundary layer is suggested by the shapes of profiles and the growth in boundary layer thickness. Critical $R e_{x}$ was $0.6-0.9 \times 10^{5}$, slightly higher that observed in the rigid fish (Fig. 6.14C), and lower than that observed for the flat plate $\left(1-3 \times 10^{5}\right.$, Figs. 6.7,6.9).

\subsubsection{Scup boundary layer thickness}

The thickness of the live scup boundary layer appeared to be similar to the Blasius boundary layer though $x / L=0.4$ at all phases (Fig. 7.8). For $x / L=0.4$ to 0.8 , boundary layer thickness grew rapidly and the profiles resembled the law of the wall profile shape more than the Blasius shape. Very little data was acquired for $x / L=0.8-1.0$ in this swimming sequence. The phases $0^{\circ}, 90^{\circ}, 270^{\circ}$, and $360^{\circ}$ were selected for plotting, rather than the phases plotted for bluefish, because the extremes in thickness occur at $\phi=0^{\circ}$ and $180^{\circ}$ (Fig. 7.9). As in bluefish, boundary layer thickness fluctuates, with a minimum 
occurring when the body surface is moving toward the fluid and a maximum when moving away. The effect is simply shifted earlier by $\sim 45^{\circ}$. The v-profiles also oscillate as observed in bluefish, with inflow as the body surface moves into the fluid and outflow when the body surface moves away from the fluid. The phase plots of boundary layer thickness (Fig. 7.9) show that the fluctuation is really only significant over the aft subregion of the fish, whereas fluctuation was significant for $x / L=0.3-0.6$ in bluefish (Fig. 7.6). This corresponds with body amplitude (Fig. 7.3). Scup exhibited the lowest body amplitude of all the fish species for the region $x / L=0.2-0.6$.

Inflected profiles are not observed at $\phi=180^{\circ}$, and it is difficult to determine anything about separation, except that the profiles look much more similar to the highspeed, turbulent, rigid case shown in Fig. $6.14 \mathrm{C}(U=48 \mathrm{~cm} / \mathrm{s})$ than to the low-speed, laminar case of Fig. $6.13 \mathrm{C}(U=19 \mathrm{~cm} / \mathrm{s})$. In the low-speed rigid case, the flow clearly separates at $x / L=0.75$, as was confirmed by reviewing the images of the flow. In the high-speed case, the flow appears to stay attached longer, which is a well-known effect of boundary layer turbulence on separation (see section 6.3.1). Although the data for the region $x / L=0.8-1.0$ at $\phi=180^{\circ}$ is very weak, what data is there is suggestive of delayed separation due to a turbulent boundary layer. Nevertheless, the outflow shown in the vprofile at $\phi=180^{\circ}$, reveals that the boundary layer is decelerating significantly.

\subsubsection{Scup boundary layer local coefficient of friction}

Fig. 7.9 shows that there is very little fluctuation in local friction in the swimming scup. The fluctuation of $C_{f x}$ in the aft sub-region is just barely significant, and, as in the bluefish, is approximately $180^{\circ}$ out of phase with the fluctuation in boundary layer thickness. The small degree of fluctuation in local friction may be due to the fact that the flow is turbulent. The extremely thin viscous sublayer of turbulent boundary layer may not be as susceptible to wall fluctuation as a laminar boundary layer, as was the boundary layer of the bluefish show in Fig. 7.5. Furthermore, body amplitude in scup was 


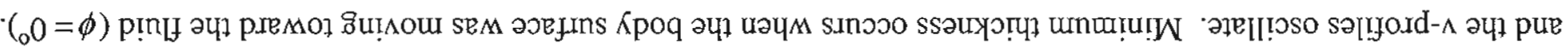

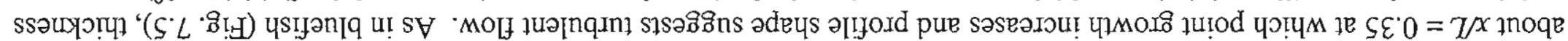
[!

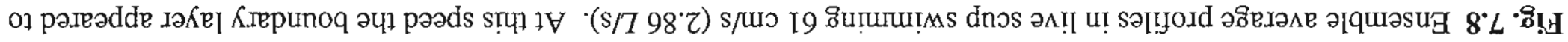
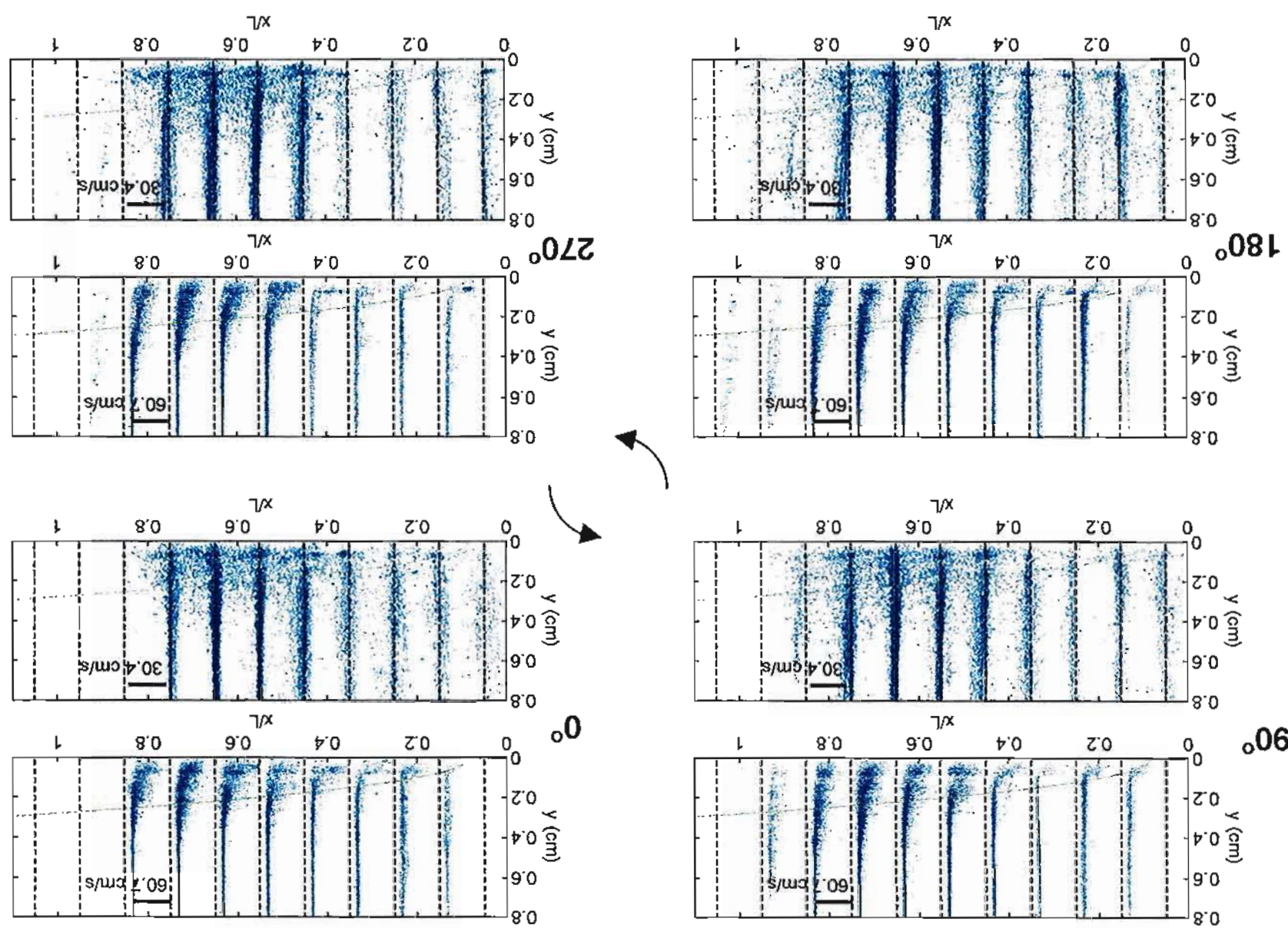


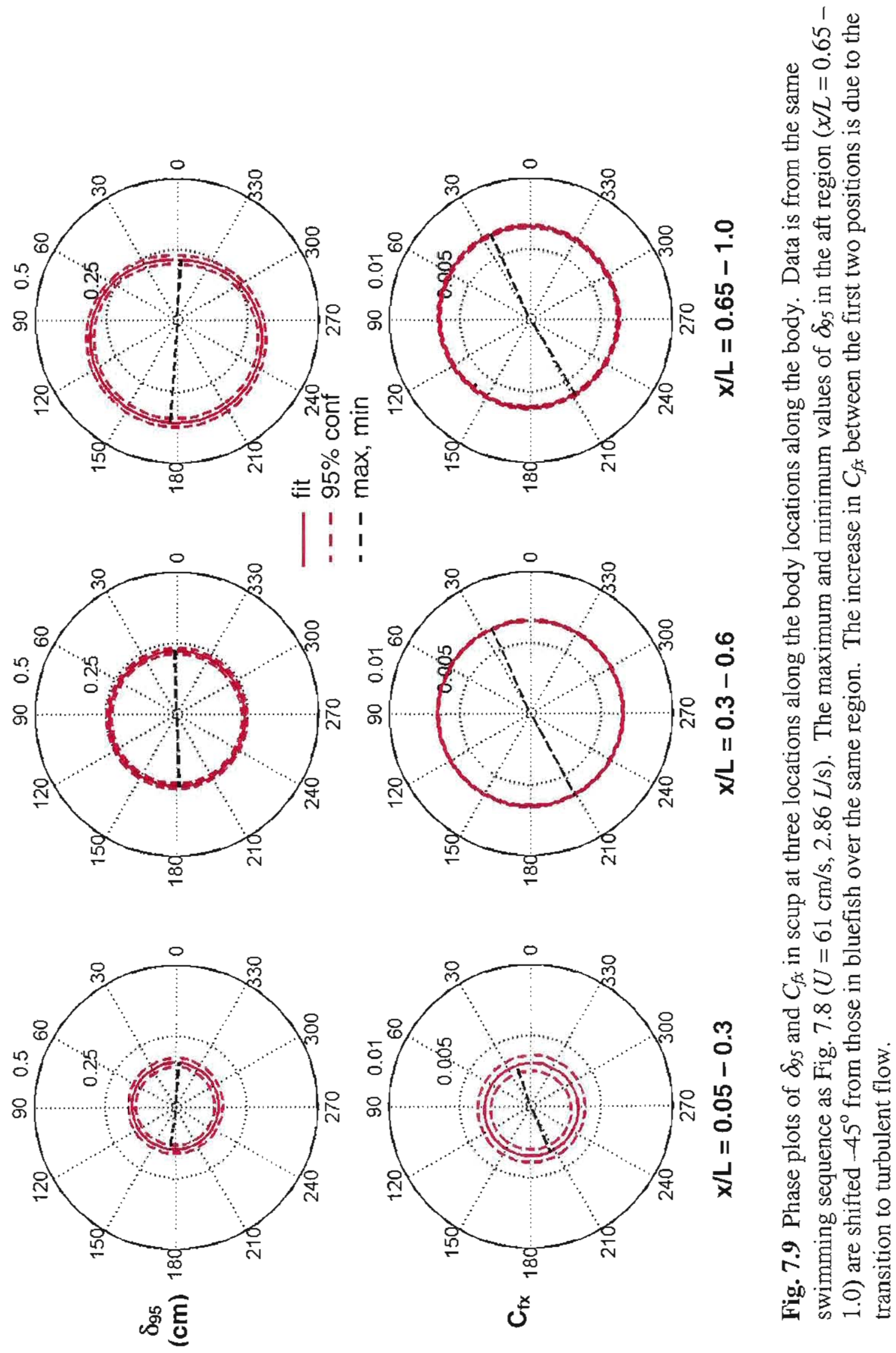

Page 207 
everywhere lower than in bluefish, and therefore it should not be surprising that fluctuations in local friction and thickness are not as dramatic. As for bluefish, the timeaveraged friction drag in the live scup was essentially the same as that determined for the rigid fish. This will be examined in more detail later in this chapter.

\subsubsection{Significance of scup boundary layer findings}

The boundary layer over a swimming scup supported the findings from the bluefish and the preliminary investigation. Boundary layer thickness, local friction and the v-profile oscillate at the same frequency of the body locomotive wave. The degree of body amplitude at any body position appears to influence the magnitude of the fluctuations. The relatively small fluctuations in scup observed in the current investigation agree extremely well with the small fluctuations observed in the preliminary investigation (Fig. 3.6) compared to the anguilliform dogfish. As in bluefish, the swimming case was found to have similar friction drag to that of the rigid-body case and no procession of the extremes of fluctuating boundary layer parameters was observed. The coefficient of friction for the swimming scup was found to be very close to that determined in the preliminary investigation.

\subsection{The eel boundary layer}

Fig. 7.10 shows an example of the boundary layer over a swimming eel. A relatively slow swimming speed is shown $(U=23 \mathrm{~cm} / \mathrm{s}, 0.65 \mathrm{~L} / \mathrm{s})$ and individual profiles suggest that the boundary layer was laminar. Profiles were highly smeared, however, and suggest a fair degree of variability or perhaps intermittent turbulence. Recall that the eel swam in the bottom boundary layer of the flume test section where spiral vorticies from the corners of the inlet and general boundary layer turbulence tripped by the inlet seam were likely to have an impact on the eel boundary layer. 


\subsubsection{Eel boundary layer thickness}

As in bluefish and scup, boundary layer thickness for the eel in Fig. 7.10 was smallest during the times that the body surface moved toward the fluid and greatest when the body surface was moving away from the fluid (Fig. 7.11). Thickness is on par with Blasius at $\phi=45^{\circ}$, but then is thicker for all other phases. Separation appears to be delayed more clearly than in bluefish or scup at $\phi=225^{\circ}$, when the body surface has been retreating from the fluid. Recall that in bluefish, inflected profiles were observed at $\phi=$ $225^{\circ}$. In scup the data was weak, but suggested delayed separation. The data is weak in the aft-most region of the eel, but not as weak as in the scup example, and the boundary layer appears to be attached. The rigid-body case (Fig. 6.13D), on the other hand, clearly becomes inflected around $x / L=0.8$, appears to separate and then reattach. Slight fluctuation of the v-profiles is apparent in a manner similar to bluefish and scup. Phase plots (Fig. 7.11) surprisingly show the greatest degree of fluctuation in thickness at the middle sub-region, $x / L=0.3-0.6$, and littie to no significant fluctuation at the fore and aft sub-regions. Extremes are located at phases similar to those seen in bluefish. Yet again, the fluctuation in boundary layer thickness shows a correspondence with body amplitude. Eel exhibited slightly higher body amplitude than scup over the mid-body and slightly lower body amplitude over the aft sub-region. As in scup, fluctuations were small.

\subsubsection{Eel boundary layer local coefficient of friction}

The same pattern and small magnitude of fluctuation observed in boundary layer thickness was observed in local friction on a swimming eel (Fig. 7.11). Time-averaged local friction was the same as for the rigid-body case. 


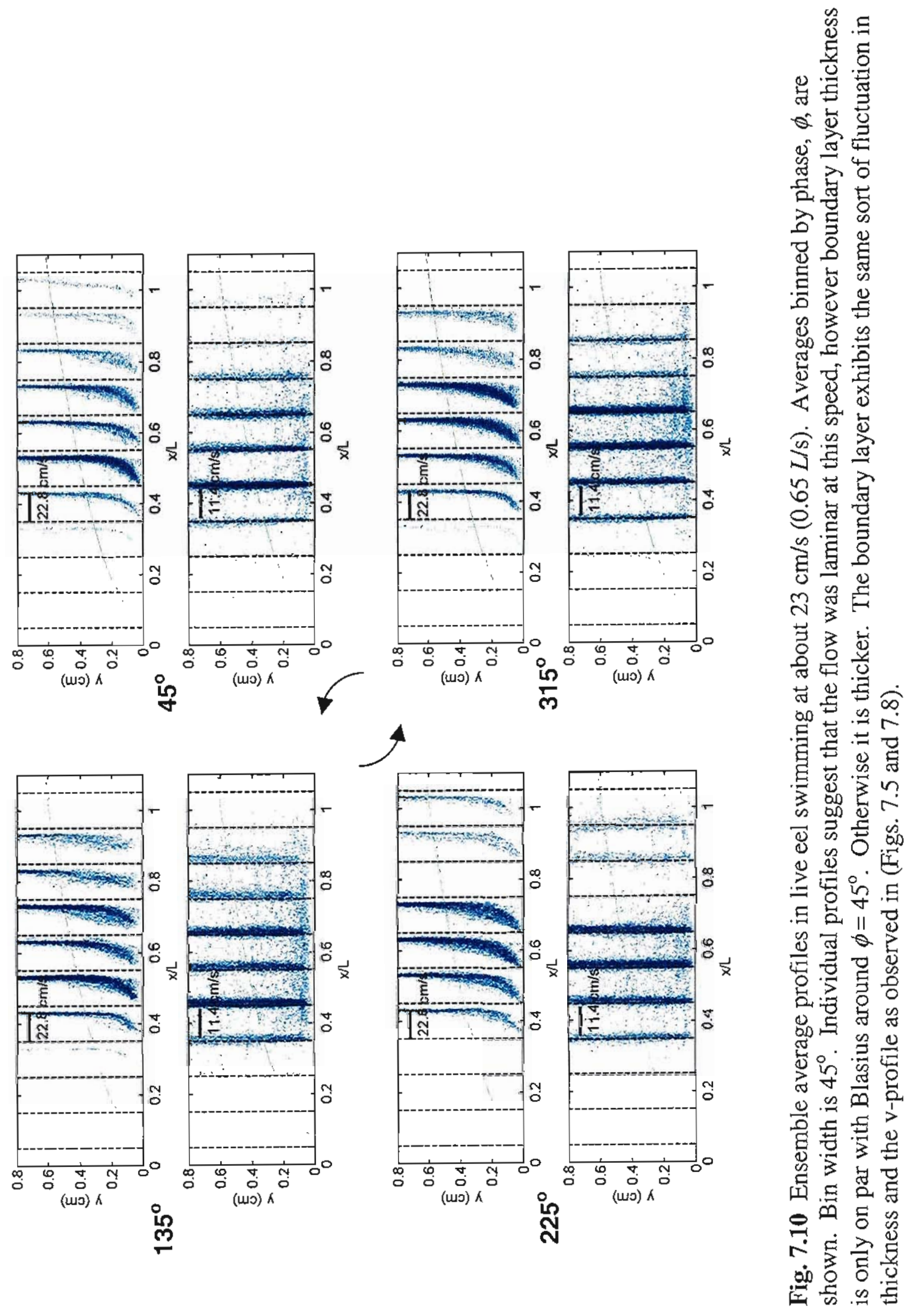

Page 210 


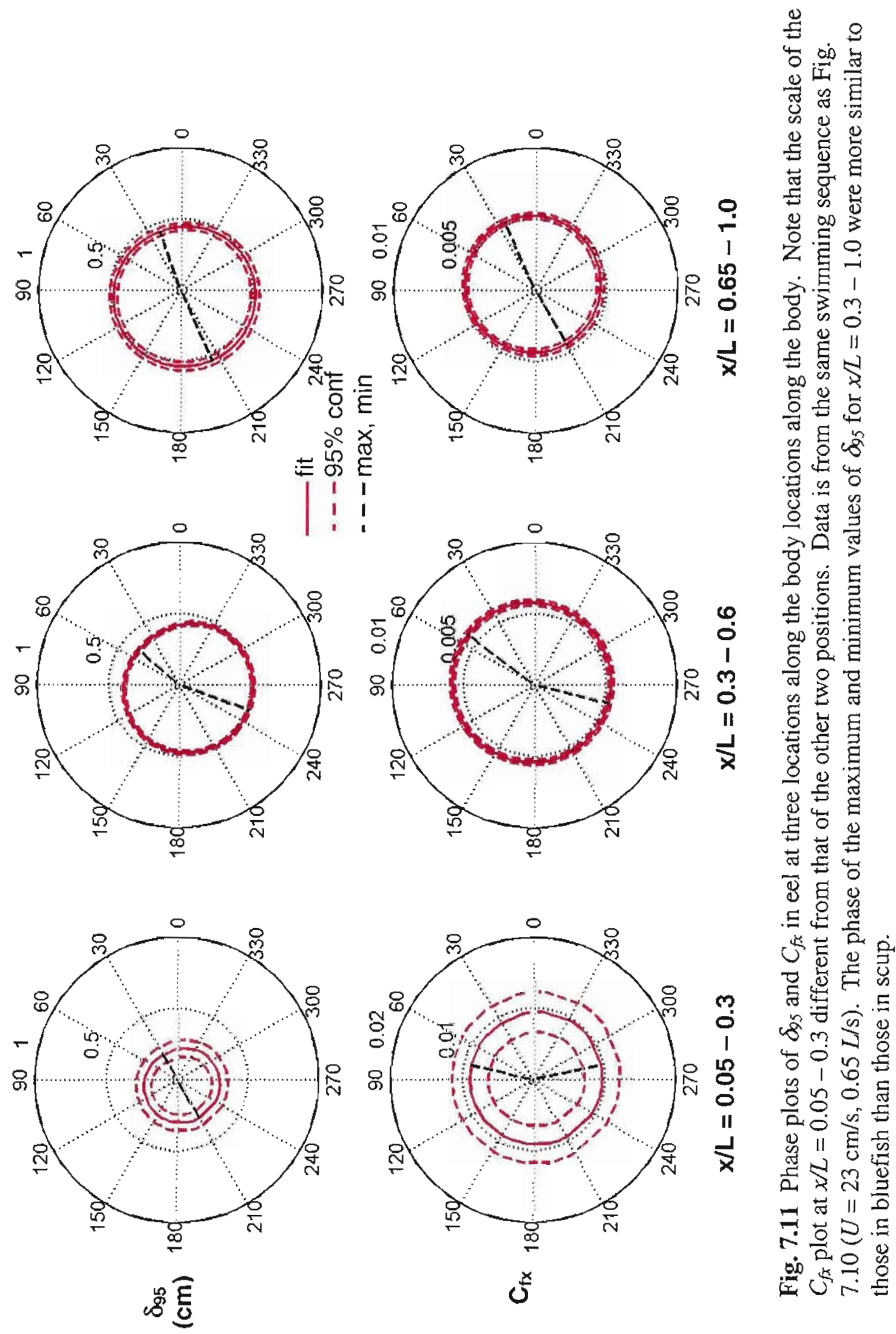

Page 211 


\subsubsection{Significance of eel boundary layer findings}

The eel boundary layer exhibited the same general characteristics of fluctuation in boundary layer thickness and local friction as seen in scup and bluefish. Again these appear to be linked to body amplitude and were small in keeping with the relatively small body amplitude observed in eel. In this way, the eel did not show itself to be similar to the dogfish of the preliminary investigation except for some weak evidence of procession of the extremes of thickness and local friction. However, the procession was of much smaller magnitude than that suggested in dogfish. Furthermore, local friction was found to be basically the same as in the rigid-body case. Body amplitude data is not available for the dogfish of the preliminary investigation because there was no nearfield camera, nevertheless, the findings here suggest that the dogfish experiments should be repeated to determine whether it truly represents such a unique case, or if perhaps aspects of the dogfish data were errant.

\subsection{The mackerel boundary layer}

Fig. 7.12 shows an example of the boundary layer over a swimming mackerel. A relatively high swimming speed is shown $(U=112 \mathrm{~cm} / \mathrm{s}, 3.68 \mathrm{~L} / \mathrm{s})$ and individual profiles suggest that the boundary layer was turbulent over the entire body. Considerable scatter in the data is present, but significant trends in boundary layer thickness and local friction were observed.

\subsubsection{Mackerel boundary layer thickness}

The boundary layer in Fig. 7.12 is much thicker than a Blasius boundary layer and the profiles resemble the shape of a turbulent profile. Significant fluctuation in thickness occurred at the mid-body, and the aft-most sub-region (Fig. 7.13). The fluctuations are of 
similar magnitude as in bluefish and the extremes fall at similar phase (Figs. 7.6, 7.7). This fits with the finding that body wave amplitude in mackerel was the greatest and most similar to that of bluefish (Fig. 7.3). In addition, inspection of the scaled sideview images (Fig. 4.1) shows that mackerel and bluefish have the most similar body shape except for the taller peduncle in bluefish.

A second, slower speed example of the boundary layer in mackerel $(U=56 \mathrm{~cm} / \mathrm{s}$, $1.72 \mathrm{~L} / \mathrm{s}$ ) suggests the occurrence of inflected profiles (Fig. 7.14) as observed in bluefish (Fig. 7.5, $\phi=225^{\circ}$ ). Mackerel seemed to prefer swimming at high speeds, so it was difficult to get a laminar case for the mackerel. The data in Fig. 7.14 is weak, but the profiles at $\phi=225^{\circ}$ are thick, highly decelerating, laminar-like and show some evidence of being inflected between $x / L=0.6$ and 0.9 . By contrast, the profiles at $\phi=45^{\circ}$ are much thinner and attached.

\subsubsection{Mackerel boundary layer local coefficient of friction}

The same pattern of fluctuation observed in boundary layer thickness was observed in local friction (Fig. 7.13), but the degree of fluctuation in local friction was quite small and slightly greater at the forward region of the fish. The lower magnitude fluctuations may be due to a similar mechanism as suggested for the turbulent boundary layer case of the scup, where the fluctuation of $C_{f x}$ was not of similar relative magnitude to that of boundary layer thickness. Time-averaged local friction was slightly lower than the rigid-body case.

\subsubsection{Significance of mackerel boundary layer findings}

The mackerel completes the apparent correspondence between body wave amplitude and the degree of fluctuation in boundary layer thickness. The mackerel boundary layer also exhibits similarities to the bluefish boundary layer, including 


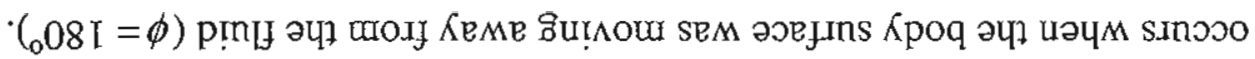

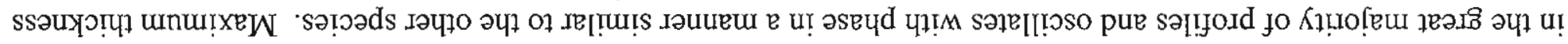

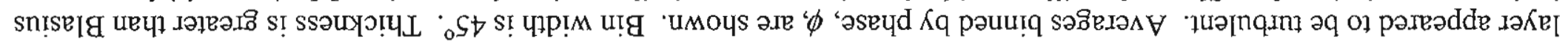

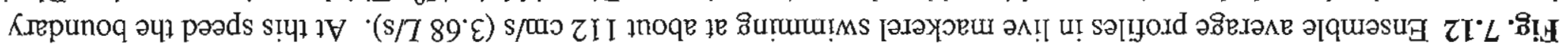
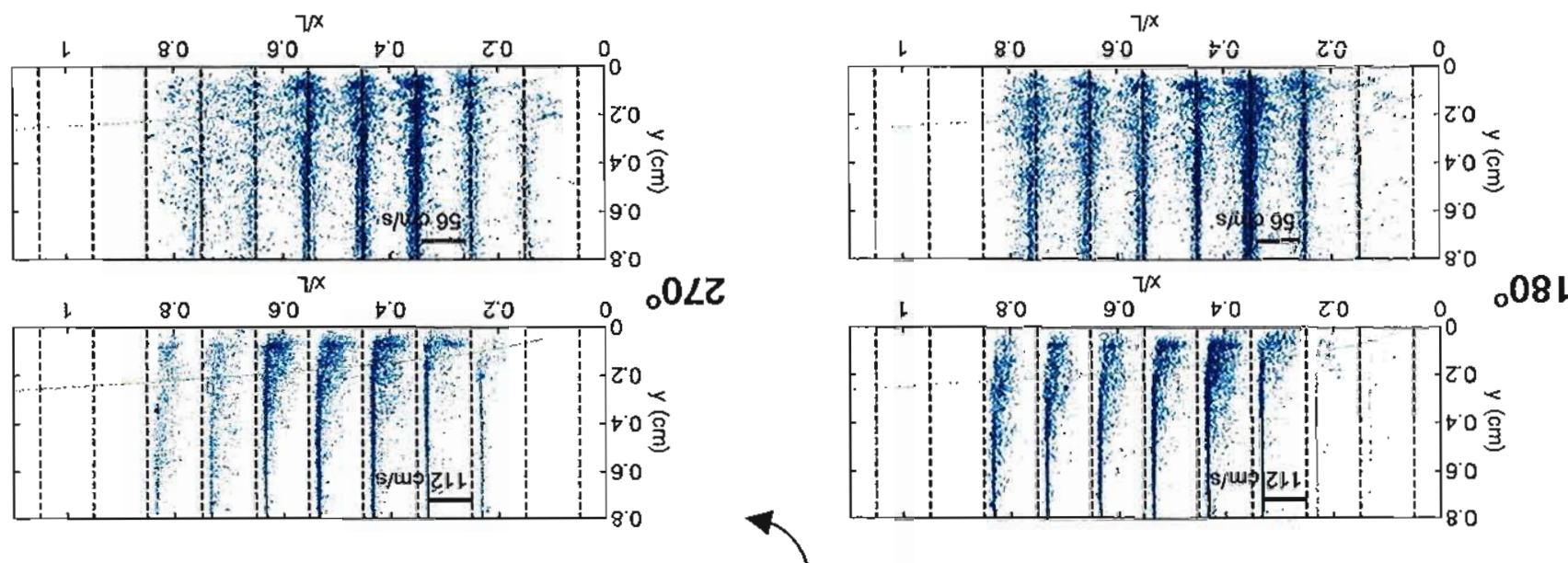

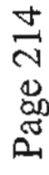
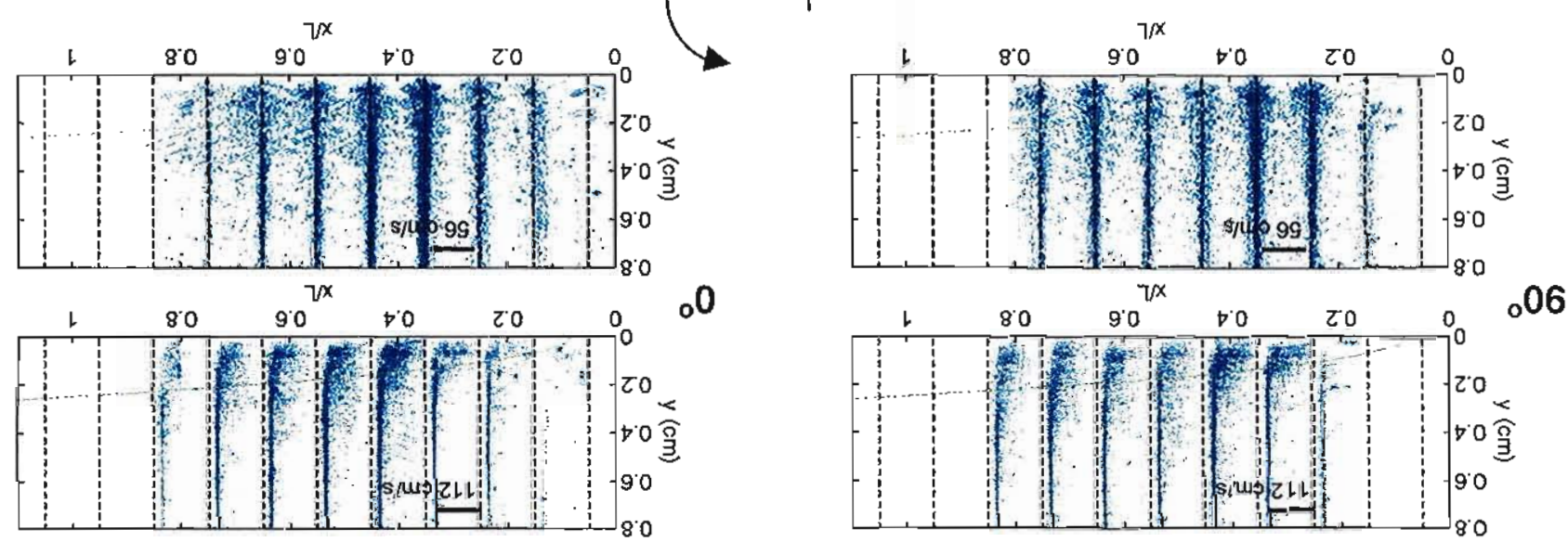


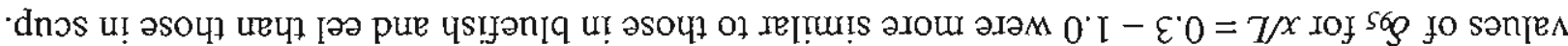

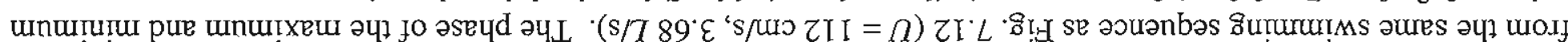

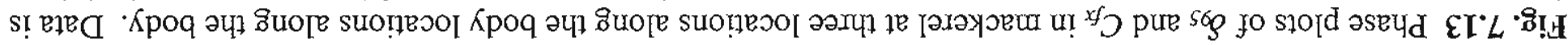
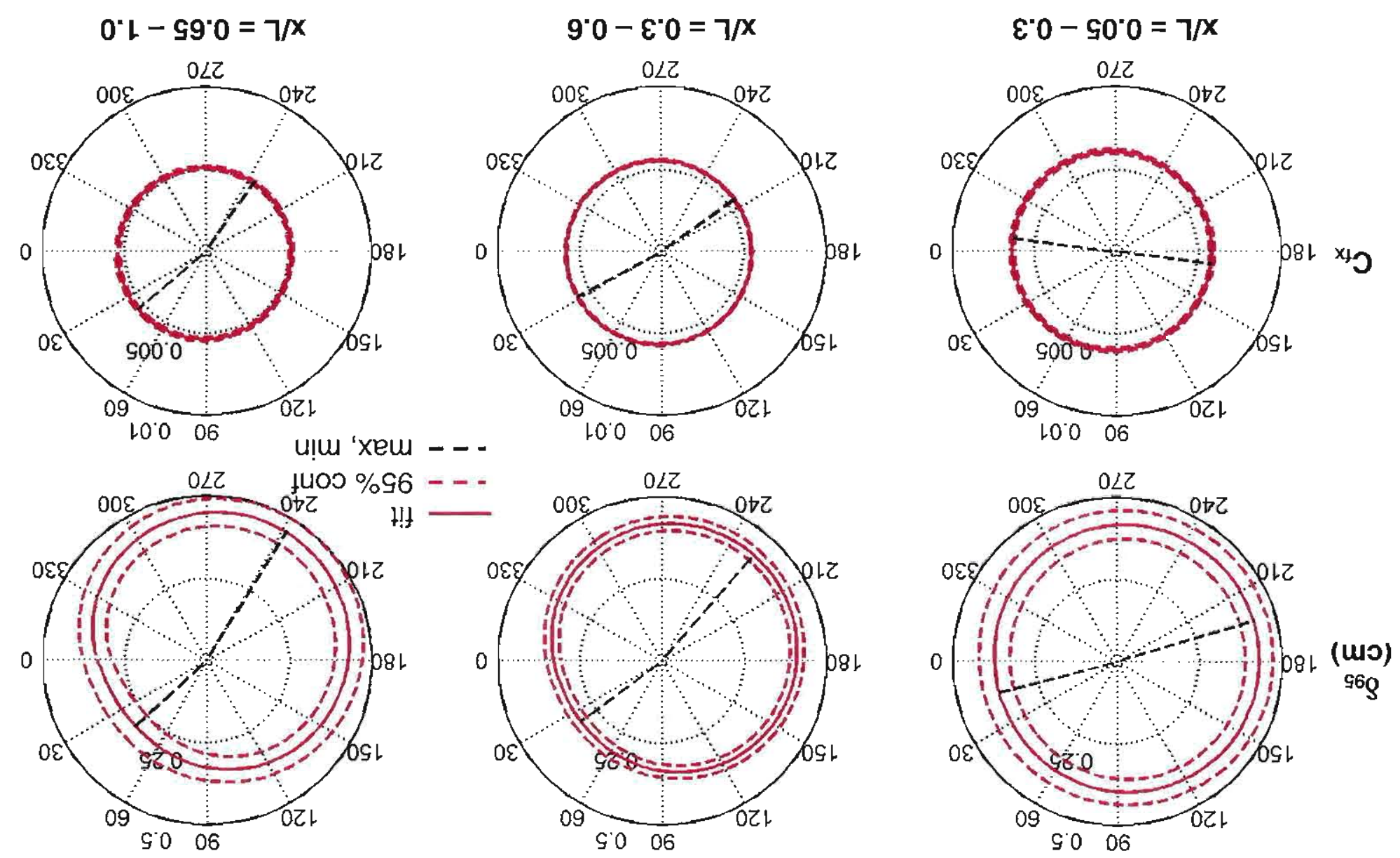


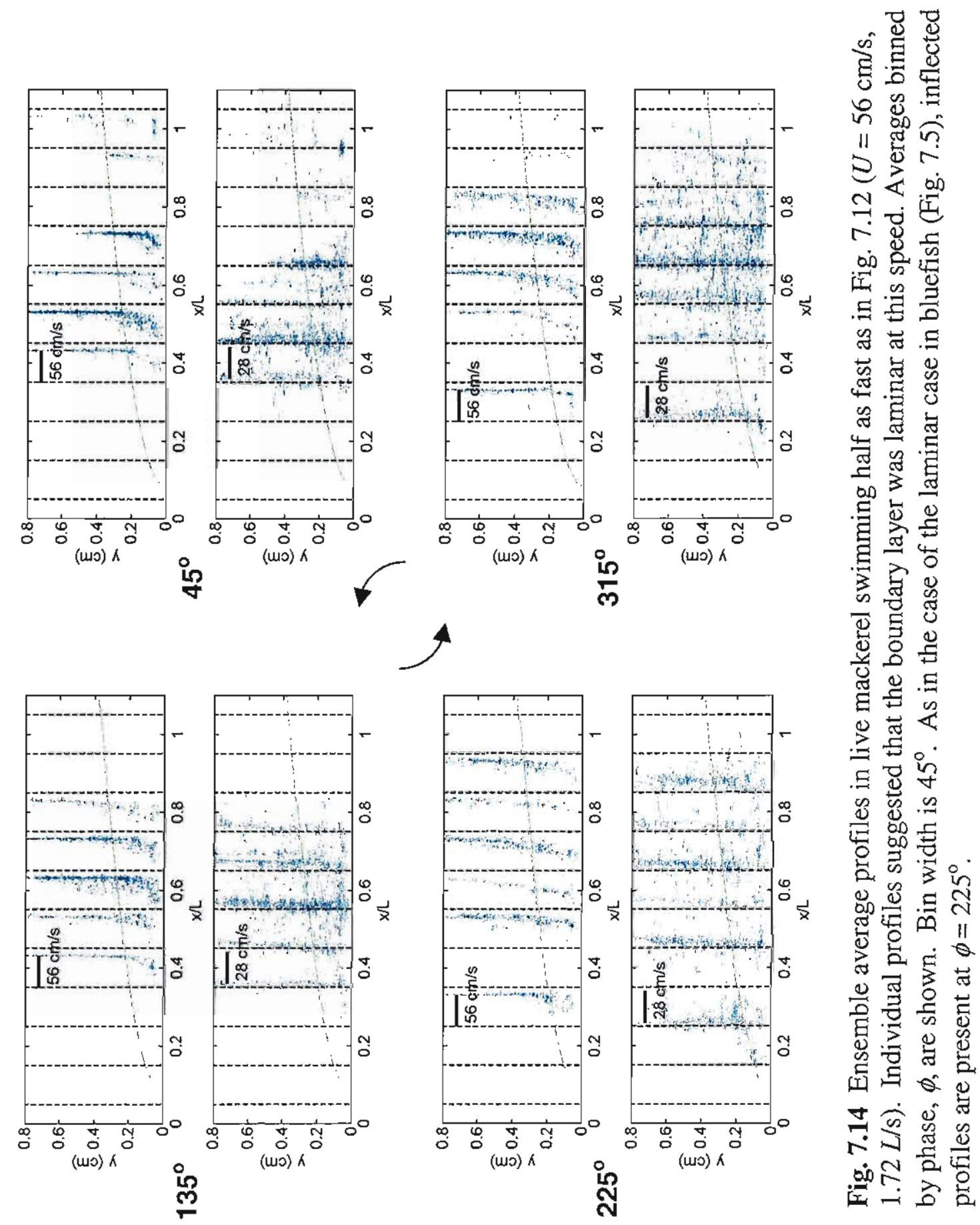

Page 216 
evidence of incipient separation when the body surface moves away from the fluid. The boundary layer is then stabilized at later phases, when the surface moves toward the fluid. The similarities seem to be tied to similarities in body wave amplitude and general body shape.

\subsection{Drag and power in swimming fish}

\subsubsection{Comparison of local friction in rigid and swimming fish}

Ultimately, the most interesting parameter that can be determined from the boundary layer profiles is friction drag. The relative local coefficient of friction, $C_{f x R}$, was calculated for swimming sequences of all four species at various speeds. As in the rigid fish, sequences were split into two groups. Those with boundary layers that appeared to be laminar and those that appeared to be turbulent. It was shown during the analysis of the flat plate that this was necessary. Fig. 7.15 shows the summary plots of $C_{f x R}$ for rigid-body and live fish experiments for each species. In general the plots show that the friction drag on rigid fish is essentially the same as the friction drag on the swimming fish of the same species. The friction for laminar cases tends to be higher than theoretical laminar flat plate friction and very close to the friction measured in the flat plate experiments. The friction for turbulent cases tends to be higher than theoretical turbulent flat plate friction, and once again close to the turbulent cases of the flat plate experiment. In general, the laminar cases in all four species exhibited similar $C_{f x R}$.

\subsubsection{Total friction coefficients and total friction drag on swimming fish}

Fig. 7.16 shows the plot of the total friction coefficient for swimming sequences of all four species. The laminar cases and turbulent cases form two groupings on the plot. The values for scup for laminar speeds agree well with the findings of the preliminary investigation (Fig. 3.13). Tables 7.1 and 7.2 list all of the swimming sequences used to 

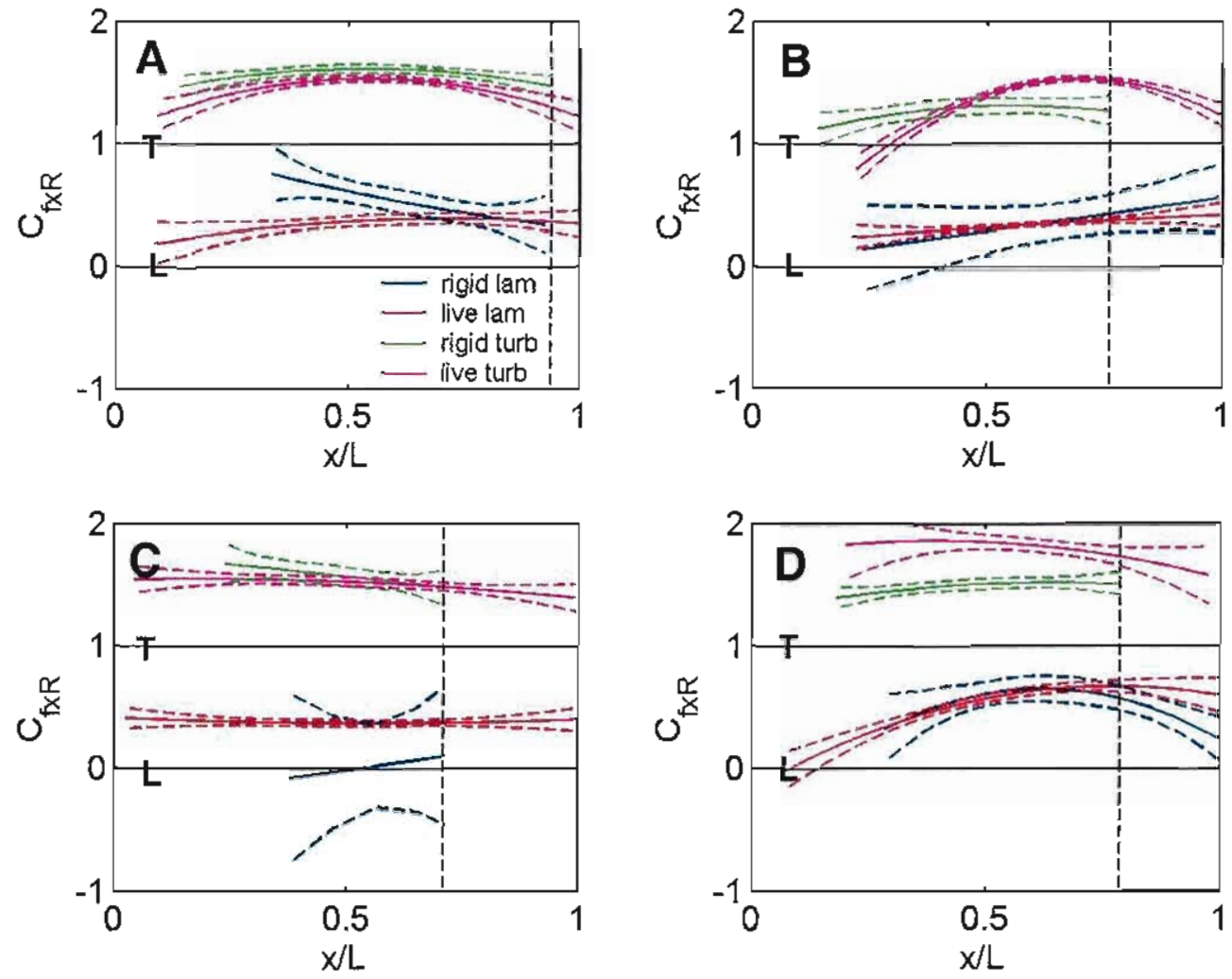

Fig. 7.15 A comparison of the relative coefficients of friction, $C_{f x R}$, with $95 \%$ confidence limits for live fish and rigid-body cases of different fish species: (A) mackerel, (B) bluefish, (C) scup, and (D) eel. The black dotted line marks the approximate separation point. Data beyond the separation point is for cases in bluefish and eel where flow did not appear to separate until close to the trailing edge. As in Fig. 6.15 the data from each species were divided into two groups depending on whether the boundary layer was laminar (lam) or turbulent (turb). In general, the local friction on a swimming fish was not significantly different from the local friction on the same rigid-fish, except in the forward half of the swimming mackere] where it is lower than in the rigid-body case. ' $T$ ' marks the line for theoretical turbulent flat plate friction, and ' $L$ ', laminar. 


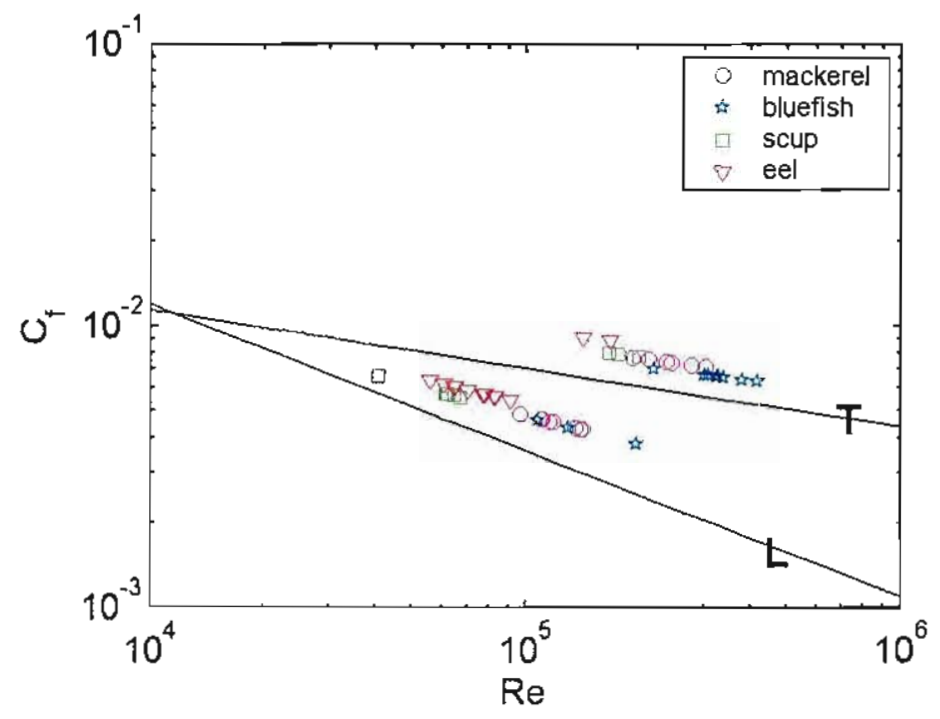

Fig. 7.16 Total coefficients of friction for all species of live fish compared to the curves representing the coefficient of friction for laminar (' $L$ ') and turbulent (' $T$ ') flow over a flat plate with the same distribution of area with length. Points between the curves for laminar and turbulent flow are live fish cases where individual profiles suggested laminar flow. The points above the curve for turbulent flow are cases in which profiles suggested turbulent flow. 
Table 7.1 Friction data for swimming sequences with a laminar boundary layer

\begin{tabular}{|c|c|c|c|c|c|c|c|c|c|}
\hline 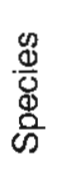 & $\begin{array}{l}\text { Length, } L \\
(\mathrm{~m})\end{array}$ & $U(\mathrm{~m} / \mathrm{s})$ & $U(L / s)$ & $\mathrm{Re}$ & $C_{f}$ & $\begin{array}{c}\text { Total } \\
\text { surface } \\
\text { area }\left(\mathrm{m}^{2}\right)\end{array}$ & $\begin{array}{c}\text { Total } \\
\text { friction } \\
\text { drag (N) }\end{array}$ & $\begin{array}{c}\text { Upper- } \\
\text { bound } \\
\text { pressure } \\
\text { drag }(\mathrm{N})\end{array}$ & $\begin{array}{l}\text { Upper- } \\
\text { bound } \\
\text { total drag } \\
\text { (N) }\end{array}$ \\
\hline \multirow{10}{*}{$\begin{array}{l}\frac{\bar{\Phi}}{\frac{1}{0}} \\
\frac{y}{0} \\
\frac{\pi}{\mathbb{N}}\end{array}$} & 0.331 & 0.366 & 1.11 & $9.76 \mathrm{E}+04$ & 0.00488 & 0.02720 & 0.00910 & 0.00820 & 0.01730 \\
\hline & 0.331 & 0.419 & 1.27 & $1.12 E+05$ & 0.00466 & 0.02720 & 0.01140 & 0.01027 & 0.02167 \\
\hline & 0.325 & 0.425 & 1.31 & $1.11 E+05$ & 0.00467 & 0.02620 & 0.01132 & 0.01020 & 0.02152 \\
\hline & 0.331 & 0.438 & 1.32 & $1.17 E+05$ & 0.00459 & 0.02720 & 0.01228 & 0.01107 & 0.02335 \\
\hline & 0.325 & 0.448 & 1.38 & 1.17E+05 & 0.00458 & 0.02620 & 0.01236 & 0.01114 & 0.02350 \\
\hline & 0.325 & 0.456 & 1.40 & $1.20 E+05$ & 0.00456 & 0.02620 & 0.01274 & 0.01148 & 0.02422 \\
\hline & 0.325 & 0.457 & 1.41 & $1.20 \mathrm{E}+05$ & 0.00455 & 0.02620 & 0.01274 & 0.01148 & 0.02422 \\
\hline & 0.325 & 0.525 & 1.62 & $1.38 \mathrm{E}+05$ & 0.00434 & 0.02620 & 0.01606 & 0.01447 & 0.03053 \\
\hline & 0.331 & 0.538 & 1.63 & 1.44E+05 & 0.00428 & 0.02720 & 0.01724 & 0.01554 & 0.03278 \\
\hline & 0.325 & 0.541 & 1.66 & $1.42 E+05$ & 0.00430 & 0.02620 & 0.01690 & 0.01523 & 0.03213 \\
\hline \multirow{3}{*}{ 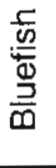 } & 50 & 0.246 & 0.55 & $1.08 \mathrm{E}+05$ & 0.00464 & 0.07280 & 0.01052 & 0.00933 & 0.01985 \\
\hline & 0.450 & 0.456 & 1.01 & $2.00 \mathrm{E}+05$ & 0.00381 & 0.07280 & 0.02940 & 0.02607 & 0.05547 \\
\hline & 0375 & 0.384 & 1.02 & $1.31 \mathrm{E}+05$ & 0.00436 & 0.05060 & 0.01668 & 0.01479 & 0.03147 \\
\hline \multirow{6}{*}{$\frac{2}{3}$} & 0.212 & 0.188 & 0.89 & $4.06 E+04$ & 0.00663 & 0.01946 & 0.00234 & 0.00285 & 0.00519 \\
\hline & 0.225 & 0.290 & 1.29 & $6.21 E+04$ & 0.00571 & 0.02200 & 0.00538 & 0.00655 & 0.01193 \\
\hline & 0.212 & 0.284 & 1.34 & $6.14 \mathrm{E}+04$ & 0.00573 & 0.01946 & 0.00462 & 0.00562 & 0.01024 \\
\hline & 0.212 & 0.288 & 1.36 & $6.22 E+04$ & 0.00571 & 0.01946 & 0.00472 & 0.00575 & 0.01047 \\
\hline & 0.212 & 0.299 & 1.41 & $6.46 \mathrm{E}+04$ & 0.00564 & 0.01946 & 0.00502 & 0.00611 & 0.01113 \\
\hline & 0.212 & 0.312 & 1.47 & $6.74 \mathrm{E}+04$ & 0.00555 & 0.01946 & 0.00538 & 0.00655 & 0.01193 \\
\hline \multirow{12}{*}{ 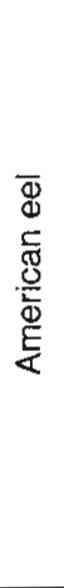 } & 0.420 & 0.193 & 0.46 & $7.74 \mathrm{E}+04$ & 0.00574 & 0.02060 & 0.00226 & 0.00049 & 0.00275 \\
\hline & 0.375 & 0.175 & 0.47 & $6.48 \mathrm{E}+04$ & 0.00608 & 0.01634 & 0.00157 & 0.00034 & 0.00191 \\
\hline & 0.375 & 0.176 & 0.47 & $6.51 E+04$ & 0.00607 & 0.01634 & 0.00158 & 0.00034 & 0.00192 \\
\hline & 0.420 & 0.207 & 0.49 & $8.27 E+04$ & 0.00562 & 0.02060 & 0.00252 & 0.00055 & 0.00307 \\
\hline & 0.350 & 0.174 & 0.50 & $5.60 E+04$ & 0.00638 & 0.01424 & 0.00141 & 0.00031 & 0.00172 \\
\hline & 0.375 & 0.191 & 0.51 & $7.07 E+04$ & 0.00591 & 0.01634 & 0.00181 & 0.00040 & 0.00221 \\
\hline & 0.350 & 0.189 & 0.54 & $6.08 E+04$ & 0.00621 & 0.01424 & 0.00162 & 0.00035 & 0.00198 \\
\hline & 0.420 & 0.228 & 0.54 & $9.13 E+04$ & 0.00544 & 0.02060 & 0.00298 & 0.00065 & 0.00363 \\
\hline & 0.374 & 0.227 & 0.61 & $7.80 E+04$ & 0.00572 & 0.01626 & 0.00246 & 0.00054 & 0.00300 \\
\hline & 0.374 & 0.228 & 0.61 & $7.85 E+04$ & 0.00571 & 0.01626 & 0.00248 & 0.00054 & 0.00302 \\
\hline & 0.375 & 0.248 & 0.66 & $9.17 E+04$ & 0.00543 & 0.01634 & 0.00280 & 0.00061 & 0.00341 \\
\hline & 0.352 & 0.246 & 0.70 & $8.36 \mathrm{E}+04$ & 0.00560 & 0.01440 & 0.00250 & 0.00055 & 0.00305 \\
\hline
\end{tabular}


Table 7.2 Friction data for swimming sequences with a turbulent boundary layer

\begin{tabular}{|c|c|c|c|c|c|c|c|c|c|}
\hline 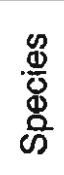 & $\begin{array}{l}\text { Length, L } \\
\text { (m) }\end{array}$ & $U(\mathrm{~m} / \mathrm{s})$ & $U(L / s)$ & $\mathrm{Re}$ & $C_{f}$ & $\begin{array}{c}\text { Total } \\
\text { surface } \\
\text { area }\left(\mathrm{m}^{2}\right)\end{array}$ & $\begin{array}{c}\text { Total } \\
\text { friction } \\
\text { drag }(\mathrm{N})\end{array}$ & $\begin{array}{c}\text { Upper- } \\
\text { bound } \\
\text { pressure } \\
\text { drag }(\mathrm{N})\end{array}$ & $\begin{array}{l}\text { Upper- } \\
\text { bound } \\
\text { total drag } \\
(N)\end{array}$ \\
\hline \multirow{6}{*}{$\begin{array}{l}\bar{\Phi} \\
\bar{\Phi} \\
\overline{0} \\
\frac{0}{2} \\
\sum\end{array}$} & 0.325 & 0.746 & 2.30 & $1.95 E+05$ & 0.00773 & 0.02620 & 0.05760 & 0.00271 & 0.06031 \\
\hline & 0.325 & 0.826 & 2.54 & $2.16 E+05$ & 0.00760 & 0.02620 & 0.06960 & 0.00328 & 0.07288 \\
\hline & 0.325 & 0.917 & 2.82 & $2.40 E+05$ & 0.00747 & 0.02620 & 0.08420 & 0.00397 & 0.08817 \\
\hline & 0.325 & 0.952 & 2.93 & $2.49 E+05$ & 0.00742 & 0.02620 & 0.09020 & 0.00425 & 0.09445 \\
\hline & 0.325 & 1.070 & 3.29 & $2.80 E+05$ & 0.00727 & 0.02620 & 0.11200 & 0.00528 & 0.11728 \\
\hline & 0.307 & 1.180 & 3.84 & $3.05 E+05$ & 0.00717 & 0.02340 & 0.11920 & 0.00562 & 0.12482 \\
\hline \multirow{8}{*}{ 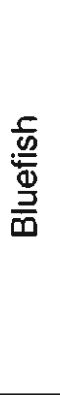 } & 0.435 & 0.531 & 1.22 & $2.23 E+05$ & 0.00706 & 0.06820 & 0.06940 & 0.00312 & 0.07252 \\
\hline & 0.450 & 0.706 & 1.57 & $3.10 E+05$ & 0.00667 & 0.07280 & 0.12420 & 0.00558 & 0.12978 \\
\hline & 0.435 & 0.728 & 1.67 & $3.04 E+05$ & 0.00669 & 0.06820 & 0.12360 & 0.00555 & 0.12915 \\
\hline & 0.450 & 0.774 & 1.72 & $3.39 E+05$ & 0.00657 & 0.07280 & 0.14680 & 0.00660 & 0.15340 \\
\hline & 0.435 & 0.781 & 1.80 & $3.27 E+05$ & 0.00661 & 0.06820 & 0.14080 & 0.00633 & 0.14713 \\
\hline & 0.450 & 0.873 & 1.94 & $3.82 E+05$ & 0.00643 & 0.07280 & 0.18320 & 0.00823 & 0.19143 \\
\hline & 0.435 & 0.996 & 2.29 & $4.18 E+05$ & 0.00633 & 0.06820 & 0.22000 & 0.00989 & 0.22989 \\
\hline & 0.375 & 0.959 & 2.56 & $3.29 E+05$ & 0.00660 & 0.05060 & 0.1 & 0.00707 & 0.16447 \\
\hline \multirow{3}{*}{ 윽 } & 0.225 & 0.830 & 3.69 & $1.78 E+05$ & 0.00793 & 0.02200 & 0.06120 & 0.00461 & 0.06581 \\
\hline & 0.212 & 0.783 & 3.69 & $1.69 E+05$ & 0.00799 & 0.01946 & 0.04880 & 0.00367 & 0.05247 \\
\hline & 0.225 & 0.948 & 4.21 & $2.04 E+05$ & 0.00777 & 0.02200 & 0.07840 & 0.00590 & 0.08430 \\
\hline \multirow[t]{2}{*}{ 离 } & 0.350 & 0.447 & 1.28 & $1.44 E+05$ & 0.00910 & 0.01424 & 0.01330 & 0.00008 & 0.01338 \\
\hline & 0.350 & 0.531 & 1.52 & $1.71 E+05$ & 0.00889 & 0.01424 & 0.01832 & 0.00011 & 0.01843 \\
\hline
\end{tabular}


produce Fig. 7.16. The tables list fish length, swimming speed, Reynolds number, the coefficient of friction, estimated surface area, and the total friction drag. The coefficient of friction for scup determined in the preliminary investigation is reported in Table 3.1 to be 0.0071 . The value determined in the current investigation is 0.0058 . The latter value is the result of thousands of boundary layer profiles sampled over a greater portion of the surface of the fish, and is therefore expected to be more accurate.

\subsubsection{Estimated pressure drag and an upper-bound total drag}

Tables 7.1 and 7.2 also include an upper-bound estimate for total drag on a swimming fish. Experimental results published by Hoerner (1958) (Figs. 4 and 5, and Eqs. 2 and 6 of his Chapter VI) show the ratio of friction drag to pressure drag on rigid, streamlined bodies as a function of thickness ratio, $t / c$, where $t$ is the maximum body thickness and $c$ is the chord, or body length in this case. The streamlined bodies presented by Hoerner ranged from airfoils to elliptical sections with thickness ratios and positions of maximum thickness in the same range as those observed in fish. As in this investigation, Hoerner treated laminar and turbulent cases separately. Table 7.3 lists measured thickness ratios and the fraction of the total drag representing friction drag as predicted by the data and models presented by Hoemer. The low thickness ratio of eel $(t / c=0.05)$ suggests that friction drag in the rigid eel is about $82 \%$ of the overall drag at laminar speeds. Therefore, only $18 \%$ is attributed to pressure drag. For the other three fish thickness ratios range from $0.12-0.15$ and friction drag is predicted to be $45-53 \%$ of the overall drag in the rigid-body case. At turbulent speeds, the friction drag is predicted to be $93-99 \%$ of the overall drag for all four species. Based on the observation that separation is somewhat delayed in swimming fish, these percentages may potentially represent upper-bound values of pressure drag on a swimming fish. The values were then used to estimate the upper-bound, total drag on the fish listed in Tables 7.1 and 7.2. In bluefish, DuBois et al. (1974) estimated drag using pressure taps on live swimming specimens and theoretical flat plate friction. They found the drag on a 
Table 7.3 The percentage of overall drag due to friction drag based on thickness ratio as predicted by Hoerner (1958) for similarly shaped, rigid, streamlined bodies

\begin{tabular}{|l|c|c|c|c|}
\hline Species & $\begin{array}{c}\text { position of } \\
\text { maximum } \\
\text { thickness } \\
(X / L)\end{array}$ & $\begin{array}{c}\text { thickness } \\
\text { ratio, } / \mathrm{C}\end{array}$ & $\begin{array}{c}\text { \% friction } \\
\text { drag, } \\
\text { laminar } \\
\text { cases }\end{array}$ & $\begin{array}{c}\text { \% friction } \\
\text { drag, } \\
\text { turbulent } \\
\text { cases }\end{array}$ \\
\hline Mackerel & 0.37 & 0.13 & 52.6 & 95.5 \\
Bluefish & 0.33 & 0.12 & 53.0 & 95.7 \\
Scup & 0.41 & 0.15 & 45.1 & 93.0 \\
American eel & 0.37 & 0.05 & 82.1 & 99.4 \\
\hline
\end{tabular}

Table 7.4 Approximate transitional length Reynolds numbers

\begin{tabular}{|l|c|c|c|}
\hline \multicolumn{1}{|c|}{ Subject } & $\begin{array}{c}\text { Inlet barrier } \\
\text { grid type }\end{array}$ & $\begin{array}{c}\text { Re } \mathrm{e}_{\mathrm{x}} \text { critical, } \\
\text { rigid case } \\
\left(\times 10^{5}\right)\end{array}$ & $\begin{array}{c}\text { Re } \text { critical, } \\
\text { swimming } \\
\text { case }\left(x 10^{5}\right)\end{array}$ \\
\hline Flat plate & blank & 2.6 & $\mathrm{NA}$ \\
Flat plate & all sizes & 1.0 & $\mathrm{NA}$ \\
Mackerel & medium & $0.5-1.0$ & $1.4-1.9$ \\
Bluefish & large & $0.4-0.9$ & $1.0-2.0$ \\
Scup & medium & $0.2-0.4$ & $0.6-1.8$ \\
American eel & small & $0.5-1.0$ & $0.9-1.4$ \\
\hline
\end{tabular}


bluefish $\sim 58 \mathrm{~cm}$ in length to be $1.6 \mathrm{~N}$ at $1.8 \mathrm{~m} / \mathrm{s}$. Table 7.2 shows that the drag on a bluefish $44 \mathrm{~cm}$ in length to be $0.22 \mathrm{~N}$ at $1.0 \mathrm{~cm} / \mathrm{s}$. If surface area, and therefore drag, is

scaled by $L^{2}$, linear regression of scaled drag vs. $U^{2}$ predicts a drag of $1.4 \mathrm{~N}$ in the case reported by DuBois et al. (1974). This is good agreement considering the uncertainties in both estimates caused by integrating relatively low resolution wall shear stress and pressure distributions over the complicated three-dimension surface of the fish.

For reference, Table 7.4 summarizes transitional $R e_{x}$ for swimming fish, rigid fish and the flat plate experiments. Transition in live swimming fish was observed to occur at slightly higher $R e_{x}$ than in rigid fish, and at values similar to that observed in the flat plate with inlet barriers in place. This suggests that the boundary layer is somewhat stabilized by the swimming motion, as was observed by Taneda and Tomonari (1974) for a waving plate.

\subsubsection{Power requirements and available muscle power}

Table 3.1 of the preliminary investigation reports the estimated power needed to overcome friction drag in smooth dogfish and scup. The table has been updated since its first publication (Anderson et al., 2001a) to better represent measurements of muscle performance in scup in the literature. In addition, data from existing studies of fish muscle were used to estimate power availability in the smooth dogfish (section 3.3.6). In the current investigation, however, the combination of upper-bound estimates for total drag (Tables 7.1 and 7.2) and higher swimming speeds observed, make it possible to conduct a more interesting analysis of the ratio of available muscle power to the required muscle power to overcome drag. Recall that in prolonged steady swimming, fish use primarily their red ('slow') muscle to power swimming (Swank and Rome, 2000; Rome et al., 1992). Swank and Rome (2000) and Rome et al. (1992) found that scup, of similar size to those used here, do not recruit white ('fast') muscle below about $80 \mathrm{~cm} \mathrm{~s}^{-1}$ at $20 \mathrm{C}$. Hence, power output in prolonged steady swimming is generally reported relative to red 
muscle mass (i.e. $\mathrm{W} / \mathrm{kg}$ red muscle). Ideally, one would like to determine the power necessary to overcome drag in a swimming fish, divide it by the total red muscle mass of the same fish, and then compare it to power availability in the muscles.

The power output required to overcome drag is simply the time-averaged drag times the swimming speed. The more difficult issue is that few complete and reliable analyses of red muscle distributions in fish have been performed (Zhang et al. 1996). Therefore, the mass of red muscle in mackerel, bluefish, and eel were estimated from various reports of red muscle given for the same or similar species in the literature, as was performed for the dogfish in Table 3.1 (section 3.3.6). By contrast, the percent of red muscle in scup (2.09\% of body mass; $3.9 \%$ of muscle mass) and the lengthwise distribution of red muscle are well known (Zhang et al., 1996). A group of scombrid fishes has also been rigorously analyzed (Graham et al., 1983). That analysis unfortunately did not include the Atlantic mackerel (Scomber scombrus), but it did include the Pacific mackerel (Scomber japonicus). A detailed red muscle distribution was determined and the ratio of red muscle to body mass was found to be $6.22 \%$ (Graham et al., 1983). Greer-Walker and Pull (1975) report that the percentage of red muscle at the position $x / L=0.67$ in Atlantic mackerel is $18.8 \%$. This is nearly identical to what was found in Pacific mackerel at that position (19.2\%). Therefore, the value $6.22 \%$ was used here for Atlantic mackerel. A rough massing of the muscle of an Atlantic mackerel suggested that this is about $9-12 \%$ of the muscle mass.

Estimating total red muscle mass is especially difficult for bluefish and American eel (Anguilla rostrata). Full red muscle distributions were not found in the literature. The only data found were (1) a red muscle mass ratio of $18.6 \%$ at the caudal peduncle in bluefish (Freadman, 1979), and (2) a ratio of $8.8 \%$ at $x / L=0.67$ in the European eel (Anguilla Anguilla) (Greer-Walker and Pull, 1975). These values cannot be taken as the percentage of red muscle to body mass, instead, some mass distribution must be assumed. The choice was between Pacific mackerel and scup. Since the mackerel exhibits a high 
Table 7.5 Power requirements based on overall, upper-bound drag, laminar cases

\begin{tabular}{|c|c|c|c|c|c|c|c|c|c|c|c|}
\hline $\begin{array}{l}\frac{\mathscr{8}}{8} \\
\mathbb{8} \\
\text { की }\end{array}$ & $\begin{array}{c}\text { Length } \\
(\mathrm{m})\end{array}$ & $\underset{(\mathrm{m} / \mathrm{s})}{U}$ & $\underset{(L / s)}{U}$ & $\begin{array}{c}\text { Mass } \\
(\mathrm{g})\end{array}$ & $\begin{array}{c}\text { Temp } \\
\text { (C) }\end{array}$ & $\begin{array}{c}\% \text { red } \\
\text { muscle/ } \\
\text { body } \\
\text { mass }\end{array}$ & $\begin{array}{l}\text { Mass } \\
\text { Red } \\
\text { Muscle } \\
(\mathrm{g})\end{array}$ & $\begin{array}{c}\text { Upper- } \\
\text { bound total } \\
\text { drag (N) }\end{array}$ & $\begin{array}{l}\text { Power } \\
\text { required } \\
\text { (W/kg) }\end{array}$ & $\begin{array}{c}\text { Min } \\
\text { avail. } \\
\text { power } \\
(W / k g)\end{array}$ & $\begin{array}{c}\text { Max } \\
\text { avail. } \\
\text { power } \\
\text { (W/kg) }\end{array}$ \\
\hline $\begin{array}{l}\bar{\Phi} \\
\frac{\Phi}{\Phi} \\
\frac{D}{D} \\
\Sigma\end{array}$ & $\begin{array}{l}0.331 \\
0.331 \\
0.325 \\
0.331 \\
0.325 \\
0.325 \\
0.325 \\
0.325 \\
0.331 \\
0.325\end{array}$ & $\begin{array}{l}0.366 \\
0.419 \\
0.425 \\
0.438 \\
0.448 \\
0.456 \\
0.457 \\
0.525 \\
0.538 \\
0.541\end{array}$ & $\begin{array}{l}1.11 \\
1.27 \\
1.31 \\
1.32 \\
1.38 \\
1.40 \\
1.41 \\
1.62 \\
1.63 \\
1.66 \\
\end{array}$ & $\begin{array}{l}349.9 \\
349.9 \\
288.3 \\
349.9 \\
288.3 \\
288.3 \\
288.3 \\
288.3 \\
349.9 \\
288.3\end{array}$ & $\begin{array}{l}13.3 \\
13.3 \\
13.2 \\
13.3 \\
13.3 \\
13.3 \\
13.3 \\
13.4 \\
13.4 \\
13.3\end{array}$ & $\begin{array}{l}6.22 \\
6.22 \\
6.22 \\
6.22 \\
6.22 \\
6.22 \\
6.22 \\
6.22 \\
6.22 \\
6.22 \\
\end{array}$ & $\begin{array}{l}21.77 \\
21.77 \\
17.93 \\
21.77 \\
17.93 \\
17.93 \\
17.93 \\
17.93 \\
21.77 \\
17.93\end{array}$ & $\begin{array}{l}0.01730 \\
0.02167 \\
0.02152 \\
0.02335 \\
0.02350 \\
0.02422 \\
0.02422 \\
0.03053 \\
0.03278 \\
0.03213\end{array}$ & $\begin{array}{l}0.29 \\
0.42 \\
0.51 \\
0.47 \\
0.59 \\
0.62 \\
0.62 \\
0.89 \\
0.81 \\
0.97 \\
\end{array}$ & $\begin{array}{l}\text { 동 } \\
\text { o } \\
\text { 혿 }\end{array}$ & $\begin{array}{l}\frac{5}{3} \\
\text { 을 } \\
\text { 站 }\end{array}$ \\
\hline 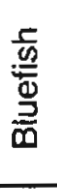 & $\begin{array}{l}0.450 \\
0.450 \\
0.375 \\
\end{array}$ & $\begin{array}{l}0.246 \\
0.456 \\
0.384 \\
\end{array}$ & $\begin{array}{l}0.55 \\
1.01 \\
1.02 \\
\end{array}$ & $\begin{array}{l}950.0 \\
950.0 \\
800.0\end{array}$ & $\begin{array}{l}21.1 \\
21.1 \\
18.0 \\
\end{array}$ & $\begin{array}{l}4.32 \\
4.32 \\
4.32 \\
\end{array}$ & $\begin{array}{l}41.04 \\
41.04 \\
34.56 \\
\end{array}$ & $\begin{array}{l}0.01985 \\
0.05547 \\
0.03147 \\
\end{array}$ & $\begin{array}{l}0.12 \\
0.62 \\
0.35\end{array}$ & $\begin{array}{l}\frac{5}{5} \\
\frac{5}{5} \\
\frac{5}{5}\end{array}$ & $\begin{array}{l}\frac{5}{5} \\
\frac{5}{5} \\
\frac{5}{5}\end{array}$ \\
\hline $\begin{array}{l}\frac{0}{7} \\
\frac{0}{2}\end{array}$ & $\begin{array}{l}0.212 \\
0.225 \\
0.212 \\
0.212 \\
0.212 \\
0.212\end{array}$ & $\begin{array}{l}0.188 \\
0.290 \\
0.284 \\
0.288 \\
0.299 \\
0.312 \\
\end{array}$ & $\begin{array}{l}0.89 \\
1.29 \\
1.34 \\
1.36 \\
1.41 \\
1.47\end{array}$ & $\begin{array}{l}182.9 \\
249.0 \\
182.9 \\
182.9 \\
182.9 \\
182.9\end{array}$ & $\begin{array}{l}23.0 \\
20.0 \\
23.0 \\
23.0 \\
23.0 \\
23.0\end{array}$ & $\begin{array}{l}2.09 \\
2.09 \\
2.09 \\
2.09 \\
2.09 \\
2.09\end{array}$ & $\begin{array}{l}3.82 \\
5.20 \\
3.82 \\
3.82 \\
3.82 \\
3.82 \\
\end{array}$ & $\begin{array}{l}0.00519 \\
0.01193 \\
0.01024 \\
0.01047 \\
0.01113 \\
0.01193\end{array}$ & $\begin{array}{l}0.26 \\
0.66 \\
0.76 \\
0.79 \\
0.87 \\
0.97\end{array}$ & $\begin{array}{l}>3 \\
>3 \\
>3 \\
>3 \\
>3 \\
>3\end{array}$ & $\begin{array}{l}>8 \\
>8 \\
>8 \\
>8 \\
>8 \\
>8\end{array}$ \\
\hline 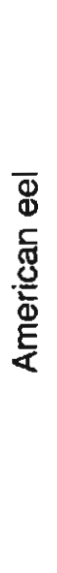 & $\begin{array}{l}0.420 \\
0.375 \\
0.375 \\
0.420 \\
0.350 \\
0.375 \\
0.350 \\
0.420 \\
0.374 \\
0.374 \\
0.375 \\
0.352\end{array}$ & $\begin{array}{l}0.193 \\
0.175 \\
0.176 \\
0.207 \\
0.174 \\
0.191 \\
0.189 \\
0.228 \\
0.227 \\
0.228 \\
0.248 \\
0.246\end{array}$ & $\begin{array}{l}0.46 \\
0.47 \\
0.47 \\
0.49 \\
0.50 \\
0.51 \\
0.54 \\
0.54 \\
0.61 \\
0.61 \\
0.66 \\
0.70\end{array}$ & $\begin{array}{l}90.3 \\
70.0 \\
70.0 \\
90.3 \\
70.0 \\
70.0 \\
70.0 \\
90.3 \\
70.0 \\
70.0 \\
70.0 \\
70.0\end{array}$ & $\begin{array}{l}20.0 \\
21.5 \\
21.5 \\
20.0 \\
18.5 \\
21.5 \\
18.5 \\
20.0 \\
18.5 \\
18.5 \\
21.5 \\
20.6\end{array}$ & $\begin{array}{l}2.04 \\
2.04 \\
2.04 \\
2.04 \\
2.04 \\
2.04 \\
2.04 \\
2.04 \\
2.04 \\
2.04 \\
2.04 \\
2.04\end{array}$ & $\begin{array}{l}1.84 \\
1.43 \\
1.43 \\
1.84 \\
1.43 \\
1.43 \\
1.43 \\
1.84 \\
1.43 \\
1.43 \\
1.43 \\
1.43\end{array}$ & $\begin{array}{l}0.00275 \\
0.00191 \\
0.00192 \\
0.00307 \\
0.00172 \\
0.00221 \\
0.00198 \\
0.00363 \\
0.00300 \\
0.00302 \\
0.00341 \\
0.00305\end{array}$ & $\begin{array}{l}0.29 \\
0.23 \\
0.24 \\
0.34 \\
0.21 \\
0.30 \\
0.26 \\
0.45 \\
0.48 \\
0.48 \\
0.59 \\
0.52\end{array}$ & $\begin{array}{l}\frac{5}{5} \\
\frac{0}{5} \\
\frac{5}{5}\end{array}$ & $\begin{array}{l}\frac{5}{3} \\
\frac{5}{5} \\
\frac{5}{5}\end{array}$ \\
\hline
\end{tabular}


Table 7.6 Power requirements based on overall, upper-bound drag, turbulent cases

\begin{tabular}{|c|c|c|c|c|c|c|c|c|c|c|c|}
\hline $\begin{array}{l}\frac{\mathscr{8}}{0} \\
\Phi \\
\mathscr{\Phi}\end{array}$ & $\begin{array}{c}\text { Length } \\
(\mathrm{m})\end{array}$ & $\underset{(\mathrm{m} / \mathrm{s})}{U}$ & $\underset{(L / s)}{U}$ & $\begin{array}{c}\text { Mass } \\
\text { (g) }\end{array}$ & $\begin{array}{c}\text { Temp } \\
\text { (C) }\end{array}$ & $\begin{array}{c}\% \text { red } \\
\text { muscle } \\
\text { body } \\
\text { mass }\end{array}$ & $\begin{array}{l}\text { Mass } \\
\text { Red } \\
\text { Muscle } \\
\text { (g) }\end{array}$ & $\begin{array}{c}\text { Upper- } \\
\text { bound total } \\
\text { drag }(\mathrm{N})\end{array}$ & $\begin{array}{c}\text { Power } \\
\text { required } \\
(\mathrm{W} / \mathrm{kg})\end{array}$ & $\begin{array}{c}\text { Min } \\
\text { avail. } \\
\text { power } \\
(W / k g)\end{array}$ & $\begin{array}{c}\text { Max } \\
\text { avail. } \\
\text { power } \\
(W / \mathrm{kg})\end{array}$ \\
\hline 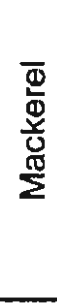 & $\begin{array}{l}0.325 \\
0.325 \\
0.325 \\
0.325 \\
0.325 \\
0.307\end{array}$ & $\begin{array}{l}0.746 \\
0.826 \\
0.917 \\
0.952 \\
1.070 \\
1.180 \\
\end{array}$ & $\begin{array}{l}2.30 \\
2.54 \\
2.82 \\
2.93 \\
3.29 \\
3.84 \\
\end{array}$ & $\begin{array}{l}288.3 \\
288.3 \\
288.3 \\
288.3 \\
288.3 \\
267.1 \\
\end{array}$ & $\begin{array}{l}13.3 \\
13.2 \\
13.3 \\
13.3 \\
13.3 \\
15.2 \\
\end{array}$ & $\begin{array}{l}6.22 \\
6.22 \\
6.22 \\
6.22 \\
6.22 \\
6.22 \\
\end{array}$ & $\begin{array}{l}17.93 \\
17.93 \\
17.93 \\
17.93 \\
17.93 \\
16.61 \\
\end{array}$ & $\begin{array}{l}0.06031 \\
0.07288 \\
0.08817 \\
0.09445 \\
0.11728 \\
0.12482 \\
\end{array}$ & $\begin{array}{l}2.51 \\
3.36 \\
4.51 \\
5.01 \\
7.00 \\
8.87\end{array}$ & $\begin{array}{l}\frac{5}{5} \\
\frac{D}{5} \\
\frac{5}{5}\end{array}$ & $\begin{array}{l}\frac{5}{3} \\
\frac{5}{5} \\
\frac{1}{5} \\
\frac{5}{5}\end{array}$ \\
\hline 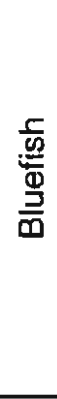 & $\begin{array}{l}0.435 \\
0.450 \\
0.435 \\
0.450 \\
0.435 \\
0.450 \\
0.435 \\
0.375\end{array}$ & $\begin{array}{l}0.531 \\
0.706 \\
0.728 \\
0.774 \\
0.781 \\
0.873 \\
0.996 \\
0.959\end{array}$ & \begin{tabular}{l|}
1.22 \\
1.57 \\
1.67 \\
1.72 \\
1.80 \\
1.94 \\
2.29 \\
2.56 \\
\end{tabular} & $\begin{array}{r}952.2 \\
1000.0 \\
952.2 \\
1000.0 \\
952.2 \\
1000.0 \\
952.2 \\
800.0 \\
\end{array}$ & $\begin{array}{l}20.5 \\
21.0 \\
20.3 \\
20.9 \\
20.4 \\
20.9 \\
20.5 \\
18.0\end{array}$ & $\begin{array}{l}4.32 \\
4.32 \\
4.32 \\
4.32 \\
4.32 \\
4.32 \\
4.32 \\
4.32 \\
\end{array}$ & $\begin{array}{l}41.13 \\
43.20 \\
41.13 \\
43.20 \\
41.13 \\
43.20 \\
41.13 \\
34.56\end{array}$ & $\begin{array}{l}0.07252 \\
0.12978 \\
0.12915 \\
0.15340 \\
0.14713 \\
0.19143 \\
0.22989 \\
0.16447 \\
\end{array}$ & $\begin{array}{l}0.94 \\
2.12 \\
2.29 \\
2.75 \\
2.79 \\
3.87 \\
5.57 \\
4.56 \\
\end{array}$ & $\begin{array}{l}\frac{5}{3} \\
\frac{5}{5} \\
\text { 돋 }\end{array}$ & $\begin{array}{l}\frac{5}{3} \\
\frac{2}{5} \\
\frac{5}{5}\end{array}$ \\
\hline 윽 & $\begin{array}{l}0.225 \\
0.212 \\
0.225\end{array}$ & $\begin{array}{l}0.830 \\
0.783 \\
0.948\end{array}$ & $\begin{array}{l}3.69 \\
3.69 \\
4.21 \\
\end{array}$ & $\begin{array}{l}249.0 \\
182.9 \\
249.0\end{array}$ & $\begin{array}{l}20.1 \\
23.0 \\
20.1\end{array}$ & $\begin{array}{l}2.09 \\
2.09 \\
2.09\end{array}$ & $\begin{array}{l}5.20 \\
3.82 \\
5.20 \\
\end{array}$ & $\begin{array}{l}0.06581 \\
0.05247 \\
0.08430\end{array}$ & $\begin{array}{l}10.50 \\
10.75 \\
15.36\end{array}$ & $\begin{array}{l}>3 \\
>3 \\
>3\end{array}$ & $\begin{array}{l}>12 \\
>12 \\
>12\end{array}$ \\
\hline 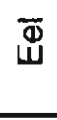 & $\begin{array}{l}0.350 \\
0.350\end{array}$ & $\begin{array}{l}0.447 \\
0.531\end{array}$ & $\begin{array}{l}1.28 \\
1.52 \\
\end{array}$ & $\begin{array}{l}70.0 \\
70.0\end{array}$ & $\begin{array}{l}18.5 \\
18.5\end{array}$ & $\begin{array}{l}2.04 \\
2.04\end{array}$ & $\begin{array}{l}1.43 \\
1.43\end{array}$ & $\begin{array}{l}0.01338 \\
0.01843 \\
\end{array}$ & $\begin{array}{l}4.19 \\
6.85 \\
\end{array}$ & 羙 & 它 \\
\hline
\end{tabular}


percentage of red muscle, the mass distribution for scup was used to prevent overestimates in bluefish and eel red muscle mass. From Zhang et al. 1996, scup muscle at $x / L=0.67$ is about $9 \%$ red muscle, and, as mentioned previously, the percentage of red muscle mass to body mass is $2.09 \%$. Using this proportion to calculate the percentage of red muscle in bluefish and eel from their percentage of red muscle at $x / L=0.67$ is the same as assuming that they have the same muscle distribution as scup. The results are red muscle mass ratios of $4.32 \%$ and $2.04 \%$ in bluefish and eel, respectively. If the bluefish is assumed to be more like the mackerel in red muscle distribution its red mass ratio becomes $6.03 \%$. Nevertheless, the lower value $(4.32 \%)$ was used to calculate a more conservative red muscle mass. Without a species specific distribution of red muscle it is difficult to judge the accuracy of these estimates.

Tables 7.5 and 7.6 show the red muscle mass and the power requirement per mass of red muscle estimated as described above for all of the swimming cases shown in Tables 7.1 and 7.2. Recall that the important differences between these tables and Table 3.1 are (1) the upper-bound, total drag was used to calculate the power requirement, not just the friction drag, and (2) the swimming speeds observed reached and surpassed maximum prolonged steady swimming speeds, i.e. speeds above which the fish tire quickly (on the order of minutes). The only exception to the latter point was mackerel, which appeared to be very comfortable swimming $3-4 \mathrm{~L} / \mathrm{s}$ for long periods of time (on the order of an hour or more). Fig. 7.17 compares the power requirements of all four fish species. All values are below $12 \mathrm{~W} / \mathrm{kg}$ except in a scup swimming $94 \mathrm{~cm} / \mathrm{s}$, at which speed recruitment of white muscle is known to occur (Rome et al., 1992). Recall that in section 3.3.6, data from power measurements for in vivo conditions in scup (Swank and Rome 2001; Rome et al., 2000; Rome and Swank, 1992) were used to estimate lower bound, red muscle power output averages of $3-12 \mathrm{~W} / \mathrm{kg}$.

Fig. 7.17A shows that, as a function of body lengths per second, mackerel require the least power output per $\mathrm{kg}$ of red muscle at a given swimming speed and that eel 

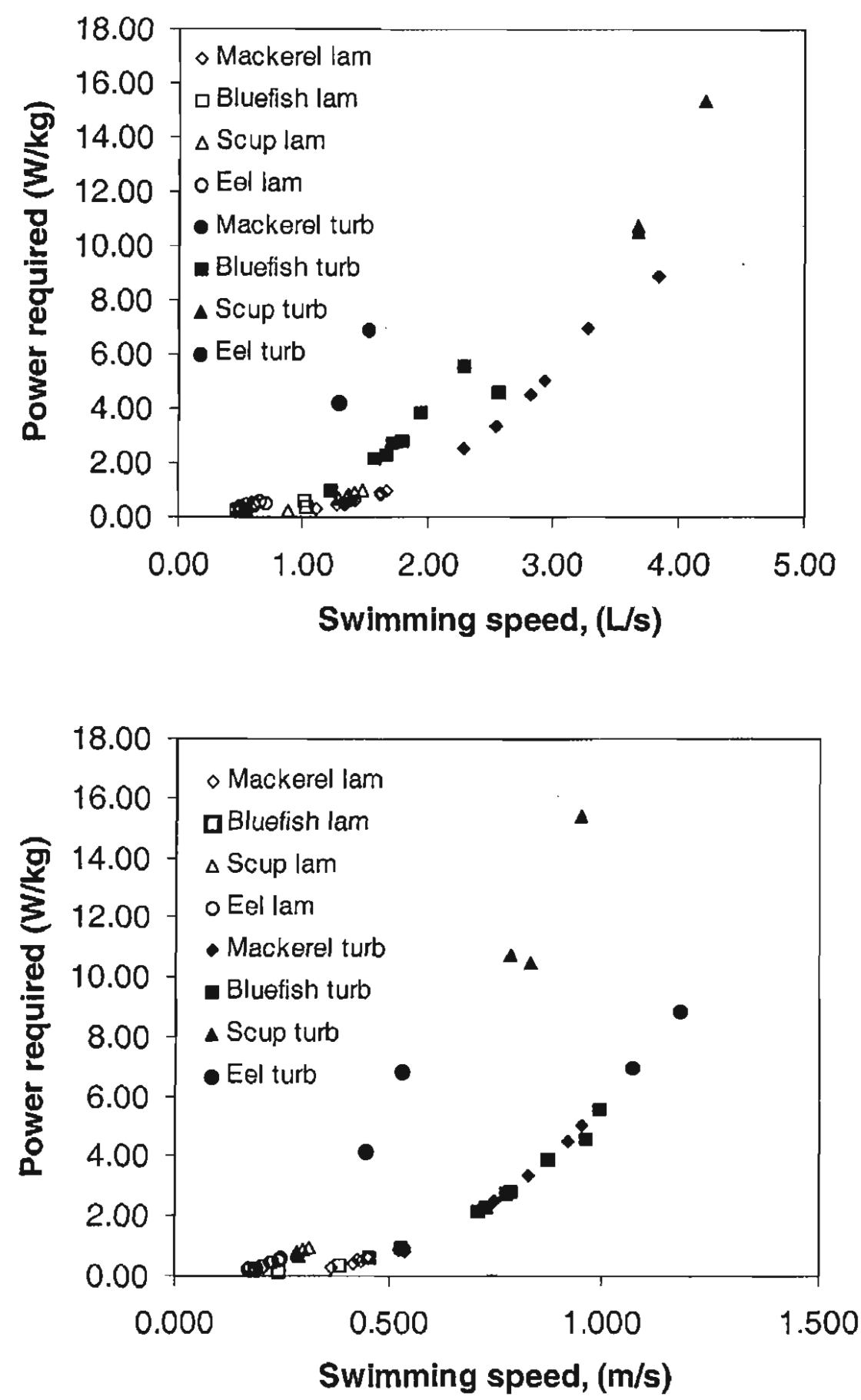

Fig. 7.17 Power per mass of red muscle required to overcome estimated upperbound drag (friction + pressure drag) in swimming fish. (A) power vs. swimming speed in body lengths/sec; (B) power vs. swimming speed in $\mathrm{m} / \mathrm{s}$. For comparison, in vivo power output for scup red muscle is estimated to be at least $3-12 \mathrm{~W} / \mathrm{kg}$ at the speeds and temperatures examined. The fastest scup, $94 \mathrm{~cm} / \mathrm{s}$, is expected to be recruiting white muscle. 
require the most. This is not surprising given the relatively large proportion of red muscle mass in mackerel. Nevertheless, bluefish appear to require a higher power output per mass of red muscle than scup, even though bluefish were assumed to have twice the mass of red muscle per body mass. This is likely an issue of scale. The large size of bluefish makes it more difficult for it to swim at high body lengths per second (Wardle, 1975). Fig. 7.17B shows the interesting result that both the bluefish and mackerel used in this investigation required essentially the same power output per mass of red muscle at any given speed.

In conclusion, these results demonstrate that even at high, prolonged swimming speeds, fish have sufficient power available in their red muscle alone to overcome fluid drag (friction and pressure drag). This is the first time this has been shown using direct measurements of friction from the boundary layer in conjunction with an upper-bound estimate of pressure drag. The observation of significantly higher burst speeds in fish are not surprising considering (1) the large mass of white muscle available and (2) the already impressive performances achieved using just $4-12 \%$ of total muscle mass. 


\section{Chapter 8}

\section{Conclusions and suggestions for future work}

This investigation has resulted in three significant advancements in the fields of fish swimming and experimental fluid dynamics: (1) the description of the major characteristics of the boundary layer in fish representing a variety of swimming styles and body shapes, (2) the measurement of friction drag and an upper-bound total drag on live swimming fish and rigid fish by the same experimental method, and (3) the development of an automatic boundary layer profiling system that enables the user to acquire and process data sets large enough for ensemble averaging and proper statistical analyses.

\subsection{The boundary layer of swimming fish}

The boundary layer of swimming fish is characterized by oscillations in thickness and instantaneous local friction predominantly driven by the transverse motion of the body surface. Fish exhibiting higher body wave amplitudes exhibit greater amplitude oscillation of thickness and local friction. Fish with similar amplitude programs, such as scup and eel, showed similar boundary layer behavior. Fish exhibiting more similar body shape, such as mackerel and bluefish also showed similar boundary layer behavior. In general, the boundary layer thins and wall shear stress increases as the body surface moves transversely toward the surrounding fluid. The boundary layer thickens and wall shear stress decreases when the surface retreats from the fluid. At swimming speeds for which the boundary layer is laminar, inflected profiles indicative of incipient separation are observed during retreat. The boundary layer sometimes appeared to separate during the middle to late retreat period at a position similar to that observed in the rigid fish. 
Over the majority of the locomotive cycle, however, separation appeared to be significantly delayed. A similar delay in separation was observed by Taneda and Tomonari (1974) in the flow over a waving plate with a wave speed just higher than the freestream flow, and likely decreases the magnitude of the pressure drag in undulatory swimming. They do not, however, mention the observance of inflected profiles. This may be due to the width and shape of the surface observed. The fish surface is not a wide, waving sheet. If any of the fish shapes studied were moved only in the transverse direction, flow would almost immediately separate near the dorsal and ventral edges of the body and affect the fluid over the entire retreating side of the fish. Nauen and Lauder (2000) have observed how the flow over the dorsal and ventral edges in chub mackerel (Scomber japonicus) interacts with small finlets running along the edges. More work is needed to determine the relationship between flow over the dorsal and ventral edges of the fish and the observed behavior of the fish boundary layer. Measurements on the centerline of a wide, waving sheet are likely to be unaffected by the separation of flow along the edge of the plate.

The boundary layer in rigid fish became turbulent at $R e_{x}$ slightly lower than observed for a flat plate in the same flume. In swimming fish, transition occurred over a range of $R e_{x}$ that generally extended above and below the critical $R e_{x}$ for the flat plate (i.e. with an inlet barrier in place). In general that range was higher than the critical $R e_{x}$ for the rigid fish (Table 7.4). Surface roughness elements on the head and structures such as the pectoral fin are likely to encourage transition in fish, but the findings above regarding Rex suggest that the motion of the fish has a weak stabilizing effect on the boundary layer. No obvious evidence of relaminarization was observed in the boundary layer profiles, although some suggestions of oscillation between laminar and turbulent flow were observed in the preliminary investigation. Interestingly, at speeds for which the boundary layer appeared to be always turbulent, oscillation in wall shear stress was relatively reduced, whereas the oscillation of boundary layer thickness did not appear to be as significantly attenuated. At 'laminar speeds', on the other hand, both wall shear 
stress and boundary layer thickness responded markedly to differences in body wave amplitude. It appears that the extremely thin viscous sublayer of the turbulent profile is less affected by the excursion of the surface.

More experimental work to better understand fish boundary layer flow is needed. First, the peduncle and tail need to be resolved more clearly. It seems that 3-dimensional flow in this region is significant and the particle tracking code struggles due to the time step limit of the current apparatus (i.e. $2.1 \mathrm{~ms}$ ). Particles travel out of the plane of the laser, or travel too far. In the case of turbulent flow, or a fluctuating separation point, particle travel needs to be much less than in a basic laminar flow. In general, the resolution of individual profiles would improve greatly with a short time step. This would facilitate taking a closer look at transition to turbulence and possible phenomena such as relaminarization.

Certainly, the boundary layer over a swimming dogfish needs to be re-examined systematically. In the preliminary investigation, only one dogfish was examined and no records of body wave amplitude were made. Dogfish experiments are necessary to confirm or reject the preliminary observations of enhanced friction drag over the rigidbody case and the procession of the extremes of boundary layer parameters through the body wave. A closer look at the effect of surface events and structures on the boundary layer is needed. It would be instructive to record the precise timings of the pectoral fin and opercular motions and analyze the impact on the boundary layer. Finally, it would be very instructive to capture the $w$-profile. This would be extremely difficult given the streamwise particle travel, however, an experiment using a beam thickness of $2 \mathrm{~mm}$, a time step of $2 \mathrm{~ms}$ and a swimming speed less than $50 \mathrm{~cm} / \mathrm{s}$ works on paper. 


\subsection{The drag on swimming fish}

The friction drag on swimming fish was determined here to be essentially the same as the friction drag on a rigid fish up to the point of separation. In cases with a laminar boundary layer, friction drag was just higher than experimental laminar flat plate drag. For turbulent cases, the drag was just higher than experimental turbulent flat plate drag. The bottom line is that in a comparative study spanning the major swimming modes no obvious friction drag reducing mechanisms were observed. In addition, no significant friction drag enhancement over rigid-body drag was observed. Drag enhancement appeared to exist in dogfish in the preliminary investigation, but it was not observed in the species of the current investigation.

In addition to friction drag, pressure drag must be investigated. The live fish boundary layer data suggests, as in the preliminary investigation, that pressure drag is likely to be relatively small since, on average, separation of flow appears to be delayed. Pressure drag on a rigid fish could be determined by measuring total force on a beam supporting a fish while the boundary layer is profiled. It is not possible to make this sort of measurement in live, swimming fish since the fish is self-propelled. Nevertheless, the upper-bound drag estimates using the models of Hoerner (1958) suggest that in eel, friction drag is always greater than $82 \%$ of the overall drag, and that in all species, when the boundary layer is turbulent, friction drag is greater than $93 \%$. In laminar cases for rigid bluefish, mackerel and scup, Hoerner's models suggest that pressure drag is similar to friction drag in magnitude. How much pressure drag might decrease in the swimming case is an interesting and important question for future work. Of course, this discussion has ignored induced drag, which is likely to occur in the swimming case, especially in the species that rely most heavily on the caudal fin for propulsion as a flapping foil.

The calculation of the upper-bound total drag combined with the relatively high swimming speeds observed in this investigation have allowed for a more definitive 
statement concerning the problematic calculations of Gray (1936) than were possible in the preliminary investigation. This investigation finds that the power required to overcome the upper-bound total drag even at high sustained swimming speeds is well within the estimates of red, or 'slow', muscle power under in vivo conditions. This suggests that Gray's drag estimate, which would have been similar to the upper-bound total drag used here, was not the problem. In an excellent investigation of swimming performance in porpoise and dolphin, Lang (1974) showed that the dramatic swimming speeds used by Gray were actually burst speeds lasting only matters of seconds, and that they were about 2.7 times sustained swimming speeds. A large mass of high power, 'fast' muscle, could easily explain a significant burst of speed (Webb, 1975). In fish, fast muscle power has been measured to have maximum optimal outputs of $15-65 \mathrm{~W} / \mathrm{kg}$ (Altringham, 1994). In vivo conditions in slow muscle resulted in estimated average power of about $1 / 3$ of the maximum optimal power. Using this as a guide, scaling power as $U^{\beta}$, and estimating the fast muscle mass to be about $85 \%$ of the total muscle mass, a fish could suddenly increase its speed to 2.2 times its maximum prolonged swimming speed. In the mackerel observed in this study that is equivalent to a burst speed of almost $9 \mathrm{~L} / \mathrm{s}$. Although fish muscle physiology may not apply directly to a marine mammal, it seems likely that Gray may have been using slow muscle measurements to model a fast muscle phenomenon as suggested by Webb (1975). In fact, Lang (1974) found that burst and sustained swimming speeds measured in porpoises were almost identical to estimated speeds based on human muscle power outputs even though he used theoretical rigid-body drag with a turbulent boundary layer, contradicting the calculations of Gray (1936). The key was taking into account the effect of muscle use duration on power output. Hence, Lang's (1974) findings suggest--as do the boundary layer profiles of fast swimming fish-that dramatic drag maintenance is not necessary to explain swimming performance in undulatory swimmers. That is not to say that there are no drag reducing mechanisms at play, but they are not on the scale of those originally suggested by Gray. 


\subsection{Improvements in boundary layer profiling}

\subsubsection{Improved temporal and spatial resolution}

Several aspects of data acquisition in this investigation could be improved to make the boundary layer profiling system a more powerful tool. First and foremost an increase in resolution in both time and space is needed. Uncertainties in the determination of $\partial u / \partial y$ at the wall were dominated by the ability to resolve flow close to the wall, especially at high speeds and when instances of three-dimensional flow appeared to be present. A custom made CCD camera with a higher pixel resolution in the $x$-direction would increase resolution of the flow near the wall. In this investigation, 1000 pixels in the $x$-direction allowed for particle tracking to about $0.1-0.2 \mathrm{~mm}$ from the wall. An increase to 4000 pixels would lead to 4 times the spatial resolution. Resolution could also be improved by making the camera field of view smaller. This can be done in the present apparatus. At higher speeds, when flow appeared to be turbulent, one could simply zero in on the very thin viscous sublayer, rather than bother with getting the entire profile. The combination of this and a higher resolution CCD chip could enhance the system's ability to determine local friction more accurately for turbulent boundary layers. Of course, having more pixels on the $\mathrm{CCD}$ chip requires a higher databus speed and more storage space. However, if temporal resolution were increased, i.e. if the time step between images were decreased significantly, the custom CCD chip could actually be made more narrow in the $y$-direction since particle travel would decrease. The particle tracking code developed in this investigation worked best for particle travel less than about 100 pixels. If particle density were high enough and there was no limit on how small the time step between images could be, the CCD could theoretically be about 200 pixels $x 4000$ pixels and acquire usable data. Such a CCD chip would actually have fewer pixels than the camera used in this investigation. 
Temporal resolution should be increased in two ways: (1) a shortened time step, as mentioned above, and (2) a higher camera frame rate. The first allows particle travel to be limited and the second allows for better time series. The second method, however, results in larger quantities of images to store and is limited by bus speed. Storage limitations for higher frame rates decrease the flexibility of the system. That is, there is less time available to get the fish in the field of view since memory runs out faster. Ultimately, the best way to deal with high frame rates is to preprocess images, extracting the necessary data in real time so that images need not be stored at all. For the boundary layer processing code detailed in this experiment, it would only be necessary to perform the edge detection, glare removal and particle centroiding. This would reduce data storage by a factor of at least 100 times, i.e. one could take data for 100 times longer. It is likely that the algorithms to perform these tasks could be 'hard-wired' into a camera operating at $30 \mathrm{~Hz}$, and higher speeds as processor speeds increase. Such systems already exist which use conventional DPIV (e.g. Dantec).

\subsubsection{Machine vision}

Even with the homemade backlighting used in this investigation, it was usually possible to track the silhouette of the fish body though basic edge detection and matching from sideview images. With a small amount of effort the backlighting could be adjusted so that tracking of the fish could be fully automated. $X$-and $Z$-axis movements of the robot could be determined from the sideview camera and fish length could be used as a cue to set a rough $Y$-position. Once the fish is visible in the nearfield camera, it is simple to position the robot in the $Y$, so that the fish body surface has the highest likelihood of passing through the boundary layer camera. Furthermore, the robot controller could be programmed to attempt to collect data from different positions along the body of the fish. This sort of machine vision system would be much better than an experimenter using a joystick at making the proper adjustments needed to maximize data acquisition. A related improvement would be the storage of the instantaneous position of the field of 
view so that knowledge of the flume profile can be used to determine the expected flow conditions at the precise location of data acquisition.

\subsection{Boundary layers in biology—a new frontier}

Virtually no investigations in experimental fluid dynamics have focused on biological boundary layers, yet the boundary layer is perhaps the most important fluid dynamic problem in biology. Mass transport (gas, biochemicals, nutrients, etc.), internal and external drag, and heat transfer all depend on the behavior and character of the boundary layer. The boundary layer profiling system developed in this investigation has the potential to open up a new field for biologists. The discovery of the boundary layer by Prandtl near the turn of the last century revolutionized fluid dynamics. As shown in this investigation, the 'discovery' of biological boundary layers has the potential to take biofluid dynamics to a new level. 


\section{References}

Adrian, R. J. (1991). Particle imaging techniques for experimental fluid mechanics. Ann. Rev. Fluid Mech. 20, 421-485.

Allen, W. H. (1961). Underwater flow visualization techniques. NOTS Technical Publication. China Lake, CA: US Naval Ordinance Test Station.

Altringham, J. D. (1994). How do fish use their myotomal muscle to swim? In vitro simulations of in vivo activity patterns. In Mechanics and physiology of animal swimming. (L. Maddock, Q. Bone, and J. M. V. Rayner, eds.). pp. 99-110. Cambridge: Cambridge University Press.

Anderson, E. J. and DeMont, M. E. (2000). The mechanics of locomotion in the squid Loligo pealei: Locomotory function and unsteady hydrodynamics of the jet and intramantle pressure. J. Exp. Biol. 203, 2851-2863.

Anderson, E. J., McGillis, W. R. and Grosenbaugh, M. A. (2001a). The boundary layer of swimming fish. J. Exp. Biol. 204, 81-102.

Anderson, E. J., Quinn, W. and DeMont, M. E. (2001b). Hydrodynamics of locomotion in the squid Loligo pealei. J. Fluid. Mech. 436, 249-266.

Bainbridge, R. (1961). Problems in fish locomotion. Symp. Zool. Soc. Lond. 5, 13-32.

Barrett, D. S., Triantafyllou, M. S., Yue, D. K. P., Grosenbaugh, M. A. and Wolfgang, M. J. (1999). Drag reduction in fish-like locomotion. J. Fluid. Mech. 392, 183-212.

Batchelor, G. K. (1967). An Introduction to Fluid Dynamics. Cambridge: Cambridge University Press.

Bechert, D. W., Hoppe, G. and Reif, W.-E. (1985). On the drag reduction of shark skin. ALAA Paper 85-0546.

Blasius, P. H. (1908). Grenzschichten in Flussigkeiten mit kleiner Reibung. Zeitschrift fur Mathematik und Physik. 56(1).

Breder, C. M. (1926). The locomotion of fishes. Zoologica 4, 159-256. 
Cheney, W. and Kincaid, D. (1994). Numerical Mathematics and Computing, $3^{\text {rd }}$ Ed. Pacific Grove, California: Brooks/Cole Publishing Company.

Coombs, S. and Montgomery, J. C. (1999). The enigmatic lateral line system. In: Comparative Hearing: Fishes and Amphibians. (A. N. Popper and R. R. Fay, eds.) Springer-Verlag, N.Y.

Coughlin, D. J., Zhang, G. and Rome, L. C. (1996). Contraction dynamics and power production of pink muscle of the scup (Stenotomus chrysops). J. Exp. Biol. 199, 27032712.

DuBois, A. B., Cavagna, G. A., and Fox, R. S. (1974). The forces resisting locomotion in bluefish. In Swimming and flying in nature. (T. Wu, C. Brokaw, and C. Brennen, eds.). pp. 541-551. New York: Plenum Press.

Ellington, C. (1984). The aerodynamics of hovering insect flight. V. A vortex theory. Phil. Trans. R. Soc. Lond. Ser. B 305, 115-144.

Falkner, V. M. and Skan, S. W. (1930). Some approximate solutions of the boundary layer equations. Aero. Res. Coun. R. \& M. No. 1314.

Fish, F. E. (1993). Power output and propulsive efficiency of swimming bottlenose dolphins (Tursiops truncatus). J. Exp. Biol. 185, 179-193.

Fish, F. E. and Hui, C. A. (1991). Dolphin swimming-a review. Mamm. Rev. 21, 181195.

Fox, R. W. and McDonald, A. T. (1992). Introduction to Fluid Mechanics, fourth edition. New York: John Wiley and Sons.

Freadman, M. A. (1979). Role partitioning of swimming musculature of striped bass, Morone saxatilis Walbaum and Bluefish, Pomatomus saltatrix L. J. Fish. Biol. 15, 417 423.

Gero, D. R. (1952). The hydrodynamic aspects of fish propulsion. Am. Mus. Novit. $1601,1-32$.

Graham, J. B., Koehrn, F. J., and Dickson, K. A. (1983). Distribution and relative proportions of red muscle in scombrid fishes: consequences of body size and relationships to locomotion and endothermy. Can. J. Zool. 61, 2087-2096.

Gray, J. (1936). Studies in animal locomotion. VI. The propulsive powers of the dolphin. J. Exp. Biol. 13, 192-199. 
Gray, J. (1968). Animal Locomotion. London: William Clowes \& Sons, Ltd.

Greer-Walker, M. and Pull, G. A. (1975). A survey of red and white muscle in marine fish. J. Fish. Biol. 7, 295-300.

Hoerner, S. F. (1958). Fluid-Dynamic Drag. New Jersey: published by the author.

Kent, J. C., DeLacy, A., Hirota, T. and Batts, B. (1961). Flow visualization and drag about a swimming fish. Fisheries Research Institute, College of Fisheries, University of Washington.

Kim, Y.-G. and Chen, C.-J. (1992). Development of digital vector velocimetry method and its application to lid driven rotating flow. Flow visualization VI, Proc. $6^{\text {th }}$ Int. Symp. Flow Visualization. (Y. Tanida and H. Miyashiro, eds.). p. 848-852. New York: Springer-Verlag.

Lang, T. G. (1974). Speed, power, and drag measurements of dolphins and porpoises. In Swimming and flying in nature. (T. Wu, C. Brokaw, and C. Brennen, eds.). pp. 553572. New York: Plenum Press.

Lighthill, M. J. (1960). Note on the swimming of slender fish. J. Fluid. Mech. 9, 305317.

Lighthill, M. J. (1969). Hydromechanics of aquatic animal propulsion-A survey. Ann. Rev. Fluid. Mech. 1, 413-446.

Lighthill, M. J. (1970). Aquatic animal propulsion of high hydrodynamic efficiency. $J$. Fluid. Mech. 44, 265-301.

Lighthill, M. J. (1971). Large-amplitude elongated-body theory of fish locomotion. Proc. Roy. Soc. B. 179, 125-138.

McKenna, S. P. and McGillis, W. R. (2002). Performance of fundamental image velocimetry techniques. Exp. Fluids. 32, 106-115.

Müller, U. K., van den Heuvel, B. L. E., Stamhuis, E. J. and Videler, J. J. (1997). Fish foot prints: morphology and energetics of the wake behind a continuously swimming mullet (Chelon labrosus Risso). J. Exp. Biol. 200, 2893-2906.

Nauen J. C and Lauder, G. V. (2000). Locomotion in scombrid fishes: morphology and kinematics of the finlets of the chub mackerel Scomber japonicus. J. Exp. Biol. 203, 2247-2259. 
Österlund, J. M. and Johansson, A. V. (1999). Measurements in a flat plate turbulent boundary layer. In the Proceedings of the First International Symposium on Turbulence and Shear Flow Phenomena (S. Banerjee and J. K. Eaton, eds.). New York: Begell House.

Patton, R. (1984). Incompressible Flow. New York: John Wiley and Sons, Inc.

Prandtl, L. (1904). Über Flussigheitsbewegung bei sehr kleiner Reibung. Verhandlung des III. Internationalen Mathematiker Kongresses. Heidelberg.

Prandtl, L. (1952). Essentials of fluid dynamics. London: Blackie and Son, Ltd.

Rayner, J. M. V. (1979a). A vortex theory of animal flight. Part 1. The vortex wake of a hovering animal. J. Fluid Mech. 91, 697-730.

Rayner, J. M. V. (1979b). A vortex theory of animal flight. Part 2. The forward flight of birds. J. Fluid Mech. 91, 731-763.

Reif, W.-E. (1982). Morphology and hydrodynamic effects of scales of fast swimming sharks. Neues. Jahrb. Geol. Paleo. 164, 184-187.

Rohr, J., Latz, M. I., Fallon, S., Nauen, J. C. and Hendricks, E. (1998a).

Experimental approaches towards interpreting dolphin stimulated bioluminescence. $J$. Exp. Biol. 201, 1447-1460.

Rohr, J. J., Hendricks, E. W., Quigley, L., Fish, F. E., Gilpatrick, J. W. and ScadinaLudwig, J. (1998b). Observations of dolphin swimming speed and strouhal number. SPAWAR Tech. Rep. 1769.

Rome, L. C., Swank, D. and Corda, D. (1993). How fish power swimming. Science 261, 340-343.

Rome, L. C. and Swank, D. (1992). The influence of temperature on power output of scup red muscle during cyclical changes in length. J. Exp. Biol. 171, 261-281.

Rome, L. C., Choi, I., Lutz, G. and Sosnicki, A. A. (1992). The influence of temperature on muscle function in fast swimming scup. I. Shortening velocity and muscle recruitment during swimming. J. Exp. Biol. 163, 259-279.

Rome, L. C., Swank, D. M. and Coughlin, D. J. (2000). The influence of temperature on power production during swimming. II. Mechanics of red muscle fibres in vivo. $J$. Exp. Biol. 203, 333-345.

Schetz, J. A. (1993). Boundary Layer Analysis. Englewood Cliffs: Simon \& Schuster. 
Schlichting, H. (1979). Boundary Layer Theory. New York: McGraw Hill.

Siller, H. A., Perkins, R. J. and Janke, G. (1993). Image analysis of oil film interferometry - a method of measuring wall shear stress distributions. Flow visualization and image analysis. (F. T. M. Nieuwstadt, ed.) p. 71-80. Netherlands: Kluwer Academic Publishers.

Sowerby, L. (1954). Secondary flow in a boundary layer. Rep. Aero. Res. Council, London No. 16832

Spedding, G. R. (1986). The wake of a jackdaw (Corvus monedula) in slow flight. J. Exp. Biol. 125, 287-307.

Spedding, G. R. (1987). The wake of a kestrel (Falco tinnunculus) in flapping flight. $J$. Exp. Biol. 127, 59-78.

Spedding, G. R., Rayner, J. M. V. and Pennycuick, C. J. (1984). Momentum and energy in the wake of a pigeon (Columbia livia) in slow flight. J. Exp. Biol. 111, 81102.

Stamhuis, E. J. and Videler, J. J. (1995). Quantitative flow analysis around aquatic animals using laser sheet particle image velocimetry. J. Exp. Biol. 198, 283-294.

Swank, D. M. and Rome, L. C. (2000). The influence of temperature on power production during swimming. I. In vivo length change and stimulation pattern. J. Exp. Biol. 203, 321-331.

Swank, D. M. and Rome, L. C. (2001). The influence of thermal acclimation on power production during swimming. I. Mechanics of scup red muscle under in vivo conditions. J. Exp. Biol. 204, 419-430.

Taneda, S. and Tomonari, Y. (1974). An experiment on the flow around a waving plate. J. Phys. Soc. Japan 36, 1683-1689.

Triantafyllou, M.S., Triantafyllou, G.S. and Gopalkrishnan, R. (1991). Wake mechanics for thrust generation in oscillating foils. Phys. Fluids A. 3, 2835-37.

Triantafyllou, G.S., Triantafyllou, M.S. and Grosenbaugh, M.A.. (1993). Optimal thrust development in oscillating foils with application to fish propulsion. J. Fluids Struct. 7, 205-24. 
Udrea, D. D., Bryanston-Cross, P. J., Moroni, M., and Querzoli, G. (2000). Particle tracking velocimetry techniques. Particle image velocimetry. (M. Stanislas, J. Kompenhans, and J. Westerweel, eds.) pp. 279-304. Netherlands: Kluwer Academic Publishers.

Van Driest, E. R. and Blumer, C. B. (1963). Boundary layer transition, free stream turbulence, and pressure gradient effects. AIAA J. 1,1303-1306.

Wardle, C. S. (1975). Limit of fish swimming speed. Nature 255, 725-727.

Webb, P. W. (1973). Effects of partial caudal-fin amputation on the kinematics and metabolic rate of underyearling sockeye salmon (Oncorhynchus nerka) at steady swimming speeds. J. Exp. Biol. 59, 565-581.

Webb, P. W. (1975). Hydrodynamics and energetics of fish propulsion. Bull. Fish. Res. Board Can. 190, 1-158.

Webb, P. W. and Weihs, D. (1983). Fish Biomechanics. New York: Praeger Publishers.

Weihs, D. (1974). Energetic advantages of burst swimming of fish. J. Theoret. Biol. 48, 215-229.

Weis-Fogh, T. and Alexander, R. M. (1977). The sustained power output from striated muscle. In Scale Effects in Animal Locomotion (T. J. Pedley, ed.), pp. 511-525. Academic Press.

Wernet, M. P. (1993). Fuzzy logic particle tracking velocimetry. Proc. SPIE-Int. Soc. Opt. Engr. (S. S. Cha and J. D. Trolinger, eds.) p. 701-708. Bellingham, WA: SPIE-The International Society for Optical Engineering.

Willert, C. E. and Gharib, M. (1991). Digital particle imaging velocimetry. Exp. Fluids 10, 181-193.

Wolfgang, M. J., Anderson, J. M., Grosenbaugh, M. A., Yue, D. K. P. and Triantafyllou, M. S. (1999). Near-body flow dynamics in swimming fish. J. Exp. Biol. 202, 2303-2327.

Zhang G., Swank, D. and Rome, L.C. (1996). Quantitative distribution of muscle fiber types in the scup. J. Morph. 29, 71-81. 


\section{Page 245}

